Monatsschr Kinderheilkd 2015.

[Suppl 1] 163:1-85

DOI 10.1007/s00112-015-3359-y

๑) Springer-Verlag Berlin Heidelberg 2015

\section{Abstracts der 41. Jahrestagung der Gesellschaft für Neonatologie und Pädiatrische Intensivmedizin (GNPI)}

\author{
21. bis 23. Mai 2015, Stuttgart - Tübingen \\ Wissenschaftliche Leitung: \\ Prof. Dr. med. Christian Poets, Tübingen \\ Martina Schürg, Tübingen \\ Dr. med. Matthias Vochem, Stuttgart \\ Oliver Hommel, Stuttgart
}

Liebe Kolleginnen und Kollegen, liebe Pflegende!

Wir begrüßen Sie sehr herzlich zur 41. Jahrestagung der Gesellschaft für Neonatologie und Pädiatrische Intensivmedizin (GNPI) in Stuttgart.

Als Kongresspräsidenten ist es uns ein Anliegen, den Weg zu weisen von der Einzelfallbeobachtung hin zur systematischen wissenschaftlichen Arbeit. Die Jahrestagung der Fachgesellschaft bietet eine gute Möglichkeit, dass junge Wissenschaftler mit erfahrenen Kolleginnen und Kollegen in Kontakt und Austausch treten. Wir sehen dabei die vielfältigen Arbeiten als Beweis, dass unsere Fachgesellschaft gut aufgestellt ist.

Aus über 180 eingereichten Beiträgen hat ein 56-köpfiges Gutachtergremium, zusammengesetzt aus allen Spezialisierungen innerhalb der Neonatologie und pädiatrischen Intensivmedizin sowie aus der Pflege, die wichtigsten und interessantesten Poster und freien Vorträge ausgewählt. Diese Arbeiten umfassen das gesamte Spektrum der beiden Teilgebiete.

Die Abstracts werden neben dieser gedruckten Version auch als Online-Supplement der Monatsschrift Kinderheilkunde im Volltextarchiv der Zeitschrift veröffentlicht und können von Ihnen unter http://link.springer.com/journal/volumesAndlssues/112 abgerufen werden. Dort wird das Supplement unter Issue 1 Supplement - May 2015 zu finden sein. So sind die Abstracts für alle Kolleginnen und Kollegen weltweit auffindbar und zitierfähig.

Sie können diese Abstracts auch jetzt schon über das W-LAN des Kongresszentrums online lesen: Die Zugangsdaten lauten: SSID: GNPI 2015; Login-Daten: Username: gnpi2015, Passwort: gnpi2015.

Der Firma Chiesi danken wir für die großzügige finanzielle Unterstützung, die die Print-Version des Abstractbands möglich gemacht hat.

Wir wünschen Ihnen einen angenehmen, informativen und anregenden Kongress und viele neue Erkenntnisse.

Ihre Tagungspräsidenten

Christian F. Poets und Matthias Vochem 


\section{Freie Vorträge}

\section{Pädiatrische Intensivmedizin: Erstversorgung}

\section{FV01 \\ Effect of different ventilation modes on return of spontaneous circulation in a newborn piglet model of asphyxia}

M. Mendler', C. Häußler', M. Hassan', M. Waitz', B. Mayer' ', H. Hummler'

${ }^{1} K$ linik für Kinder- und Jugendmedizin, Universitätsklinikum Ulm, Sektion Neonatologie und pädiatrische Intensivmedizin, Ulm, Deutschland, ${ }^{2}$ Institut für Epidemiologie und Medizinische Biometrie, Ulm, Deutschland

Background. There are no clear evidence-based recommendations on the use of different techniques of respiratory support and chest compressions (CC) during neonatal cardiopulmonary resuscitation (CPR). Objectives. To determine the effects of different ventilatory support strategies along with CC representing clinical practice on return of spontaneous circulation (ROSC) in asphyxiated newborn piglets with cardiac arrest. We hypothesized that the use of a T-Piece-resuscitator (TPR) providing positive end-expiratory pressure (PEEP) reduces time to ROSC as compared to a self-inflating bag (SIB) without PEEP. Furthermore, we explored the effects of the use of a ventilator providing inflations without synchrony to CC.

Methods. Thirty-three newborn piglets were progressively asphyxiated until asystole occurred and randomized into three groups and resuscitated according to the ILCOR guidelines. Group 1: TPR (peak inspiratory pressure (PIP)/PEEP of $25 / 5 \mathrm{cmH} 2 \mathrm{O}$, rate $30 / \mathrm{min}$ ) with inflations interposed between CC (3:1 ratio). Group 2: SIB (PIP of $25 \mathrm{cmH}_{2} \mathrm{O}$ without PEEP, rate $30 / \mathrm{min}$ ) with inflations interposed between $\mathrm{CC}$ (3:1 ratio). Group 3: ventilator (PIP/PEEP of $25 / 5 \mathrm{cmH} 2 \mathrm{O}$, rate $30 / \mathrm{min}$ ). CC were applied with a rate of $120 / \mathrm{min}$ without synchrony to inflations. Animals were supported for 120 minutes after ROSC. Primary outcome was time to ROSC. Results. All animals achieved ROSC. We found no significant difference in time to ROSC comparing the three groups [TPR: 150 (150-210) s; SIB: 150 (120-180) s; Ventilator: $180(150-345) \mathrm{s}$; Median (IQR)]. There was no difference in use of epinephrine or in blood gases or hemodynamic parameters during the 120 minutes observation time after ROSC. Conclusions. We found no significant effect on ROSC using different respiratory support strategies during CPR in the delivery room.

\section{Auswirkungen perinataler Faktoren auf die kindliche Entwicklung}

\section{FV02 \\ Körperfettanteil bei Neugeborenen von Müttern mit Gestations- diabetes (GDM)}

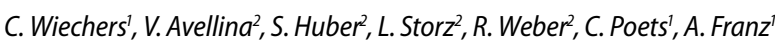

'Eberhard-Karls-Universität Universitätsklinik für Kinderheilkunde und Jugendmedizin, Neonatologie, Tübingen, Deutschland, ${ }^{2}$ Eberhard-KarlsUniversität Universitätsklinik für Kinderheilkunde und Jugendmedizin, Tübingen, Deutschland

Hintergrund. Die Inzidenz des GDM steigt und wird aktuell auf bis zu $20 \%$ geschätzt. Die maternale Hyperglykämie kann zu einer Vielzahl von kurz- und langfristigen Problemen bei den Neugeborenen führen. Unter anderem stimuliert das Überangebot maternaler Glukose die Sekretion von Insulin- und Insulin like growth factors und hierdurch die Zunahme des Fettgewebes bzw. der Körperfettmasse.

Fragestellung. Ziel war, den Körperfettanteil von eutrophen Kindern gesunder Müttern mit dem von Kindern von Müttern mit GDM zu vergleichen. Außerdem sollte geprüft werden, ob Kinder von Müttern mit GDM, die trotz Frühfütterung hypoglykäm werden, einen höheren Körperfettanteil aufweisen als solche die euglykäm bleiben.
Material und Methoden. In dieser Studie wurden von Juni 2014 bis November 2014 reife Einlings-Neugeborene (Gestationsalter: 37.-42. SSW) eingeschlossen. Der Körperfettanteil wurde mittels Air Displacement Plethysmographie (PEA POD ${ }^{\circledR}$ Infant Body Composition System, Cosmed, Rome, Italy) bestimmt. Die normalverteilten Daten sind als Mittelwert mit Standardabweichung dargestellt und mittels Zweistichproben T-Test unter der Annahme unterschiedlicher Varianz ausgewertet. Ergebnisse. Von bisher 414 angesprochenen Eltern stimmten 55\% (228/414) der Untersuchung zu. Bei 178/228 konnten Messungen durchgeführt werden und bei 144/228 ausgewertet werden. 14 Kinder waren hypotroph [Geburtsgewichts (GG)-SDS $<-1,28$ ], 103 Kinder waren eutroph $(-1,28 \leq$ GG-SDS $\leq+1,28)$, 20 Kinder hatten Mütter mit GDM, davon waren 4 hypertroph, und 11 Kinder waren hypertroph (GGSDS $>1,28)$ davon hatten 4 diabetische Mütter. 11 Kinder von Müttern mit GDM hatten Hypoglykämien in den ersten Lebensstunden. Neugeborene von Müttern mit GDM hatten im Vergleich mit eutrophen Neugeborenen gesunder Mütter ähnliche GG-SDS- [0,41 $( \pm 0,93)$ vs. $0,06( \pm 0,63) ; \mathrm{p}=0,12]$ und Körperfettanteil-Werte $[12,9( \pm 4,0) \%$ vs. 11,4 $( \pm 3,6) \% ; p=0,12]$. Neugeborene von Müttern mit GDM, die am ersten Lebenstag hypoglykäm wurden $(\mathrm{n}=11)$, unterschieden sich hinsichtlich ihres Körperfettanteils nicht von denen, die unter Frühfütterung euglykäm blieben $(n=9): 12,8( \pm 4,5) \%$ vs. $12,9( \pm 3,5) \%$; $p=0,95$.

Schlussfolgerung. Entgegen unserer Hypothese wiesen Neugeborene von Müttern mit einem Gestationsdiabetes keinen erhöhten Körperfettanteil auf. Kinder von Müttern mit GDM, die hypoglykäm wurden ließen sich nicht anhand ihres Körperfettanteils von denen unterschieden, die unter Frühfütterung euglykäm blieben.

\section{FV03}

Zum Einfluss der Körperhöhe der Mutter auf die Häufigkeit von Schwangerschafts- und Geburtsrisiken bei hypotrophen, eutrophen und hypertrophen Neugeborenen

M. Grunner', M. Voigt'2 V. Hesse', U. Wittwer-Backofen ${ }^{3}$, H. Hagenah ${ }^{4}$

'Deutsches Zentrum für Wachstum, Entwicklung und Gesundheitsförderung im Kindes- und Jugendalter, Berlin, Deutschland, ${ }^{2}$ Inst. für Perinatale Wachstumsforschung, Sievershagen, Deutschland, ${ }^{3}$ Zentrum Medizin und Gesellschaft, Freiburg, Deutschland, ${ }^{4}$ Klinikum Rotenburg, Geburtshilfe, Rotenburg, Deutschland

Zielstellung. Zwischen der Körperhöhe der Mutter und dem Geburtsgewicht gibt es einen linearen Zusammenhang. Damit liegen die Normwertkurven für relativ kleine Mütter niedriger und für relativ große Mütter höher als die Normwertkurven aller Neugeborenen. Gleichzeitig werden aber wichtige Schwangerschafts- und Geburtsrisiken auch von der Körperhöhe der Mutter beeinflusst. Es soll gezeigt werden, wie sich die Häufigkeit von Schwangerschafts- und Geburtsrisiken verändert, wenn man zur somatischen Klassifizierung der Neugeborenen einerseits die Gesamtnormwertkurve des Geburtsgewichtes verwendet und andererseits Normwertkurven unter Berücksichtigung der Körperhöhe der Mutter berechnet.

Material und Methode. Das Datenmaterial umfasst 508.926 Datensätze der deutschen Perinatalerhebung der Jahre 1998-2000 aus 8 Bundesländern. Die statistischen Auswertungen erfolgten mit dem Statistikprogrammpaket „SPSS“ im Rechenzentrum der Universität Rostock. Die Neugeborenen wurden in hypotrophe (<10. Perz.), eutrophe (10.-90. Perz.) und hypertrophe (>9o. Perz.) klassifiziert.

Ergebnisse. Klassifiziert man die Neugeborenen mit den Geburtsgewichtsperzentilkurven aller Neugeborenen, so liegt die Häufigkeit einer Plazentainsuffizienz bei relativ kleinen Müttern $(=151 \mathrm{~cm})$ bei hypotrophen Neugeborenen bei 10,1\% und bei relativ großen Müttern $(=182 \mathrm{~cm})$ bei $17,3 \%$. Benutzt man nun die auf die Körperhöhe der Mutter bezogenen Geburtsgewichtsperzentilkurven, so erhöht sich der Anteil einer Plazentainsuffizienz bei den hypotrophen Neugeborenen von Müttern $=151 \mathrm{~cm}$ auf $15,5 \%$ und fällt bei den Müttern $=182$ auf 8,4\% zurück.

Schlussfolgerung. Bei der Beurteilung vieler Schwangerschafts- und Geburtsrisiken müsste berücksichtigt werden, dass ihre Häufigkeit auch 
von der Körperhöhe der Mutter beeinflusst wird. Dieses hat dann auch Folgen für das Ursachenspektrum von Schwangerschafts- und Geburtsrisiken bei hypotophen, eutrophen und hypertrophen Neugeborenen.

\section{FV04}

\section{Maternal prepregnancy weight and neonatal outcome: does adiposity really matter?}

\section{E. Tutdibi', T. Kieren', M. Bücheler'², L. Gortner'}

'Unversitätsklinikum des Saarlandes, Klinik für Allgemeine Pädiatrie und Neonatologie, Homburg, Deutschland, ${ }^{2}$ Qualitätsbüro des Saarlandes, Saarbrücken, Deutschland

Background. Maternal prepregnancy body mass index (BMI) and weight gain during pregnancy are well-known risk factors for unfavorable perinatal outcomes. Recent epidemiological studies and meta-analyses strongly suggest deleterious impact of abnormal maternal BMI on neonatal short-term morbidity and mortality.

Objective. We thus aimed at describing the risk of preterm and term neonates born to mothers with adiposity on the background of increasing prevalence of adiposity.

Methods. We reviewed the data reported to the maternal and neonatal quality assurance program of the federal state of the Saarland (Germany). Exclusion criteria were missing maternal BMI, age under 18 years or pregravid BMI classified as underweight $(<18.5 \mathrm{~kg} \mathrm{~m}-2)$. Weight of the women in the first trimester was used to estimate pregravid BMI and study cohort was compared between normal weight $\left(18.5-29.9 \mathrm{kgm}^{-2}\right)$ and adiposity $\left(>30 \mathrm{~kg} \mathrm{~m}^{-2}\right)$. Relevant maternal and neonatal variables were defined prospectively and analyzed statistically by SPSS version 20 software.

Results. A total of 21581 pregnancies delivered from $24+0$ to $42+6$ weeks were reported between 2010 and 2012. After exclusion, 14,203 infants born to 13,500 mothers were analysed in the study. The adiposity rate was increasing significantly during the study period $(\mathrm{p}<0.027)$. The overall prevalence of maternal pregravid adiposity was $17.8 \%$ and occurred more often in German mothers, singles and smoking women. Pregnancy complications as gestational diabetes and EPH gestosis were significantly associated with adiposity $(\mathrm{p}<0.001)$. Rates of cesarean section, use if general anesthesia and induction of labor were increased in adipose women $(\mathrm{p}<0.001)$. Infants born to adipose mothers showed more often fetal distress (pathological Apgar score at 5 minutes $<7$ and umbilical artery $\mathrm{pH}<7.1$ ). In contrast to preterms, full-term newborns needed more often supplemental oxygen or endotracheal intubation in delivery room $(\mathrm{p}<0,025)$. Admission rate to NICU was increased in adipose group. Short-term pulmonal morbidity of full-term newborn was impaired in adipose cohort whereas morbidity of preterms did not differ between study groups. Perinatal mortality of preterms was 3 times higher born to adipose mothers.

Conclusion. The results of our cohort study demonstrate the different impact of pregravid adiposity on the perinatal outcome in relation to gestational age. The causal link has to be determined by further studies.

\section{FV05 \\ Analyse von maternalen Risikofaktoren für die Geburt hyper- tropher Neugeborener aus den Daten der deutschen Perinatal- erhebung}

A. Knie', M. Voigt ${ }^{2}$, V. Hesse $e^{3}$, E. Schleußner', D. Olbertz

${ }^{1}$ Klinikum der Friedrich-Schiller-Universität-Jena, Klinik für Frauenheilkunde und Geburtshilfe, Abteilung Geburtshilfe, Jena, Deutschland, ${ }^{2}$ Universität Freiburg, Zentrum für Medizin und Gesellschaft, Freiburg, Deutschland, ${ }^{3}$ Deutsches Zentrum für Wachstum, Entwicklung und Gesundheitsförderung im Kindes- und Jugendalter, Berlin, Deutschland, ${ }^{4}$ Klinikum Südstadt Rostock, Abteilung für Neonatologie und Neonatologische Intensivmedizin/Kinderschlafmedizin, Rostock, Deutschland

Ziel. Es werden die Bedeutung ausgewählter mütterlicher Merkmale (Alter, Körpergewicht, Körperhöhe, BMI, Gewichtszunahme während der Schwangerschaft, Gestationsdiabetes, Rauchverhalten, Parität, alleinstehend/nicht alleinstehend) für das Auftreten einer neonatalen Makrosomie bei Reifgeborenen analysiert.

Methode. Die Daten von 465.964 Neugeborenen der Jahre 1998-20oo aus der deutschen Perinatalerhebung von 8 Bundesländern werden analysiert. Die maternalen Daten von Kinder >95. Geburtsgewichtsperzentile $(n=46.035)$ wurden mit den von eutrophen Termingeborenen $(n=374.486)$ verglichen. Die statistische Auswertung erfolgte deskriptiv sowie mittels $\chi^{2}$-Test. Die Risikokalkulation erfolgte mittels univariater und multivariablen logistischer Regression mit dem Programmpaket „SPSS“ im Rechenzentrum der Universität Rostock.

Ergebnisse. Risikofaktoren für ein hypertrophes Neugeborenes sind (1) ein maternales Gewicht $>70 \mathrm{~kg}$ (OR 1,9 95\% CI 1,86-1,95) und mütterliche Adipositas (BMI $>30)$. (OR 2,15). (2) Ein Schwangerschaftsdiabetes (OR 2,18), (3) eine überstarke Gewichtszunahme $>19 \mathrm{~kg}$ während der Schwangerschaft (OR 2,0). (4) Mehrgebärende haben häufiger hypertrophe Neugeborene (OR 2,08). Überraschender Weise stellen das mütterliche Alter und alleinstehende Mütter keine wesentlichen unabhängigen Risikofaktoren dar.

Schlussfolgerung. Es konnten für eine mitteleuropäische Population Risikofaktoren definiert werden, die für die Primär- und Sekundärprävention von fetalen Makrosomien Ansatzpunkte geben können.

\section{FV06}

Analyse von maternalen Risikofaktoren für die Geburt hypotropher Neugeborener aus den Daten der deutschen Perinatalerhebung

\section{A. Knie? , M. Voigt ${ }^{2}$, V. Hesse ${ }^{3}$, E. Schleußner', D. Olbertz ${ }^{5}$}

${ }^{1} K$ linikum der Friedrich-Schiller-Universität-Jena, Klinik für Frauenheilkunde und Geburtshilfe, Abteilung Geburtshilfe, Jena, Deutschland, ${ }^{2}$ Universität Freiburg, Zentrum für Medizin und Gesellschaft, Freiburg, Deutschland, ${ }^{3}$ Deutsches Zentrum für Wachstum, Entwicklung und Gesundheitsförderung im Kindes- und Jugendalter, Berlin, Deutschland, ${ }^{4}$ Klinikum der Friedrich-Schiller-Universität-Jena, Klinik für Frauenheilkunde und Geburtshilfe, Direktor der Abteilung Geburtshilfe, Jena, Deutschland, ${ }^{5}$ Klinikum Südstadt Rostock, Abteilung für Neonatologie und Neonatologische Intensivmedizin/Kinderschlafmedizin, Rostock, Deutschland

Ziel. Es werden die Bedeutung ausgewählter mütterlicher Merkmale (Alter, Körpergewicht, Körperhöhe, BMI, Gewichtszunahme während der Schwangerschaft, Rauchverhalten, Parität, alleinstehend/nicht alleinstehend) für das Auftreten einer neonatalen Hypotrophie bei Reifgeborenen analysiert.

Methode. Die Daten von 465.964 Neugeborenen der Jahre 1998-20oo aus der deutschen Perinatalerhebung von 8 Bundesländern werden analysiert. Die maternalen Daten von Kinder $<5$. Geburtsgewichtsperzentile $(\mathrm{n}=45.443)$ wurden mit denen von eutrophen Termingeborenen $(n=374.486)$ verglichen. Die statistische Auswertung erfolgte deskriptiv sowie mittels $\chi_{2}$-Test. Die Risikokalkulation erfolgte mittels univariater und multivariablen logistischer Regression mit dem Programmpaket „SPSS“ im Rechenzentrum der Universität Rostock.

Ergebnisse. Maternale Risikofaktoren für ein hypotrophes Neugeborenes sind (1) Mütterliches Rauchen (OR 2,3), wobei ein klarer Dosiseffekt nachzuweisen ist. Bei mehr als 10 Zigaretten täglich steigt das Risiko auf OR 2,95; 95\% CI 2,83-3,07); (2) mütterliches Untergewicht (BMI<18,5) OR 2,08; (3) eine geringe Gewichtszunahme $<7 \mathrm{~kg}$ während der Schwangerschaft OR 1,89; 95\% CI 1,84-1,95; (4) Schwangere mit Kleinwuchs (OR 1,42 ; $95 \%$ CI 1,38-1,95) oder geringen Gewicht (OR 1,47; 95\% CI 1,46-1,53). Überraschenderweise stellen das mütterliche Alter und alleinstehende Mütter keine wesentlichen unabhängigen Risikofaktoren dar.

Schlussfolgerung. Es konnten für eine mitteleuropäische Population Risikofaktoren definiert werden, die für die Primär- und Sekundärprävention von fetalen Wachstumsstörungen Ansatzpunkte geben können. 


\section{FV07}

\section{Nabelschnur pH-Wert und APGAR-Score differenziert nach Trag-} zeit und Geburtsmodus

\section{Vollmer', A. Strauss', M. Voigt' ${ }^{2}$ D. Olbertz ${ }^{3}$, H. Hagenah ${ }^{4}$}

${ }^{1}$ Klinik für Gynäkologie und Geburtshilfe UKSH Campus Kiel, Kiel, Deutschland, ${ }^{2}$ Institut für Perinatale Wachstumsforschung, Sievershagen, Deutschland, ${ }^{3}$ Abt. Neonatologie des Klinikums Südstadt, Rostock, Deutschland, ${ }^{4}$ Frauenklinik Geburtshilfe Agaplesion Diakonie Klinikum Rotenburg (Wümme), Rotenburg, Deutschland

Einleitung. In der Geburtshilfe werden nach der Geburt der arterielle Nabelschnur pH-Wert und der APGAR-Score zur Zustandsdiagnostik des Neugeborenen verwendet. Nabelschnur pH-Wert Interpretation nach Saling pH>7,30: normal, $\mathrm{pH}$ 7,29-7,25: reduziert, $\mathrm{pH}$ 7,24-7,20: Präazidose, $\mathrm{pH}$ 7,19-7,15: leichte Azidose, $\mathrm{pH}$ 7,14-7,10: mittelgradige Azidose, $\mathrm{pH}$ 7,09-7,05: fortgeschrittene Azidose, $\mathrm{pH}<7,04$ : schwere Azidose Analog die Zustandsdiagnostik durch den APGAR-Score: 8-10 lebensfrisch, 4-7 deprimiert, 1-3 schwer deprimiert.

Material und Methoden. Als Grundlage des Auswertungskollektives standen die Daten der deutschen Perinatalerhebung aus 8 Bundesländern von 1998-2000 (508.926 Fälle) zur Verfügung. Aus den Daten wurden Subgruppen erstellt und miteinander korreliert. $\mathrm{pH}>7,19 ; 7,19-7,15$; 7,14-7,00; <7,0 und APGAR-Score 8-10; 4-7; 1-3. <32. SSW; 40. SSW, Spontangeburt; Sectio caesarea.

Ergebnisse. Erwartungsgemäß korrelieren niedrige $\mathrm{pH}$-Werte mit niedrigen APGAR-Werten sowohl bei Frühgeburten und reifgeborenen Kindern nahezu unabhängig vom Entbindungsmodus. Jedoch ist festzustellen das in der Gruppe der Spontangeburten sowohl bei Frühgeburten als auch bei reif geborenen Kindern die Häufigkeitsverteilungen in der APGARBewertung bei $\mathrm{pH}$-Werten $>7,19$ und 7,19-7,15 nahezu identisch sind [APGAR 8-10: 70,2\% (pH 7,15-7,19) zu 69,4\% (pH>7,19)]. Bei den reif geborenen Kindern zeigen sich in der Gruppe der primären respektive sekundären Sectio caesarea ebenfalls keine großen Unterschiede. Primäre/sekundäre Sectio caesarea [APGAR 8-10: 92,4\%/92,4\% (pH 7,15-7,19) 98,2\%/97,2\% $(\mathrm{pH}>7,19)]$. Einzig bei den Frühgeburten gab es große Differenzen.

Schlussfolgerung. Der APGAR-Score wird von unterschiedlichen Berufsgruppen je nach Organisation in der Klinik, und Schwangerschaftsalter (Frühgeburten in der Regel durch Pädiater) vergeben und unterliegt damit einer unter Umständen subjektiveren Beurteilung $(\mathrm{Ge}$ burtshelfer, Kinderärzte, Anästhesisten). Der pH-Wert stößt bei Leihazidosen durch die Mutter ebenfalls an seine Grenzen (niedrige $\mathrm{pH}$ Werte beim Kind durch metabolische oder respiratorische Azidose der Mutter). Weiterführende Untersuchungen sollten unter Einbeziehung von venösem + arteriellen $\mathrm{pH}$-Wert $+\mathrm{BE}$ aus der Nabelschnur und APGAR-Bewertung, unterteilt nach Berufsgruppen, erfolgen.

\section{Neonatale Pulmonologie}

\section{FV08 \\ Polyunsaturierte Plasma-Phospholipide von Mutter-Kind- Paaren - Gestationsalterbedingte Veränderungen und Beziehung zur bronchopulmonalen Dysplasie (BPD)}

W. Bernhard', C. Engel', M. Raith', V. Koch', C. Maas', H. Abele', C. Poets', A. Franz' ${ }^{1}$ Eberhard-Karls-Universität Universitätsklinik für Kinderheilkunde und Jugendmedizin, Neonatologie, Tübingen, Deutschland, ${ }^{2}$ CPCS-Center for Pediatric Clinical Studies, Universitätsklinik für Kinderheilkunde und Jugendmedizin, Tübingen, Deutschland, ${ }^{3} U$ niversitäts-Frauenklinik, Mutter-Kind-Zentrum, Tübingen, Deutschland

Hintergrund. Docosahexaensäure (DHA), Arachidonsäure (DHA) und Cholin sind für die fetale Entwicklung aller Organe essentiell, mit exponentiell ansteigendem Bedarf ab 24 Wochen $(w)$ postmenstruellem Alter (PMA). Diese Nährstoffe werden diaplazentar im Feten angereichert, während Linolsäure (LA) im mütterlichen Organismus retiniert wird.

\section{Tab. FV08}

Lipidparameter

Korrelationskoeffizient Signifikanzniveau zu BPD (Grad 0-4)

\begin{tabular}{lll}
\hline $\begin{array}{l}\text { DHA-PC (\% von PC } \\
\text { in fetalem Plasma) }\end{array}$ & $\mathrm{r}=0,6052$ \\
\hline ARA-PC/DHA-PC-Ratio in NSP & $\mathrm{r}=-0,0060$ \\
\hline Fetomaternale EPA-PC-Ratio & $\mathrm{r}=0,48$ & $\mathrm{p}=0,0013$ \\
\hline
\end{tabular}

Plasma-Phosphatidylcholin (PC) ist wesentlicher Carrier und Indikator der DHA-, ARA- und LA-Homöostase. Postpartal sinken die Plasmakonzentrationen von Cholin, DHA-PC und ARA-PC bei Frühgeborenen zu einem unphysiologischen Entwicklungszeitpunkt auf die Hälfte intrauteriner Werte ab, wohingegen LA-PC ernährungsbedingt ansteigt.

Fragestellung. (1) Bestimmung der physiologischen Veränderungen in der PC-Homöostase von Feten und Schwangeren in Abhängigkeit vom postmenstruellen Alter (PMA); (2) Beziehung zwischen PC-Status von Fetus und Gebärender zum Geburtszeitpunkt und der Entwicklung einer BPD; (3) zu prüfen, ob Abweichungen im Fettsäureprofil von PC im Nabelschnurplasma mit BPD assoziiert sind.

Material und Methoden. Probenpaare von Nabelschnurplasma und mütterlichem Serum von 108 Schwangerschaften (24-42 wk PMA). Bestimmung der molekularen Spezies von PC mittels Tandemmassenspektrometrie. Korrelation der Lipidparameter mit dem PMA und bei Frühgeborenen <28 Wochen PMA mit dem BPD-Schweregrad.

Ergebnisse. Die mediane (25er/75er Perzentile) PC-Konzentration betrug 1,29 (o,92-1,31) mmol/1 im Nabelschnurblut, jedoch 3,33 (2,86-3,89) $\mathrm{mmol} / \mathrm{l}$ in mütterlichen Proben $(\mathrm{p}<0,0001)$. Fetale und maternale PCKonzentration und -Zusammensetzung waren direkt korreliert. Der LA-PC-Anteil im Nabelschnurblut betrug nur 26,6 $(24,6-28,5) \%$, gegenüber maternalen $49,7(46,5-52,6) \%$. Fetales ARA-PC betrug hingegen $34,9(32,4-37,6) \%$ gegenüber maternalen 17,6 (15,9-19,5)\%. Der Anteil an DHA-PC war im Nabelschnurplasma <28 w PMA niedriger als bei den Müttern. Es überstieg deren Werte erst nach einem break point von $33 \mathrm{w}$. Unter 28 w PMA korrelierten ein hoher Anteil an DHA-PC im fetalen Plasma direkt und eine hohe ARA-PC/DHA-PC-Ratio im fetalen Plasma invers mit Entwicklung und Schweregrad einer BPD. Ebenso korrelierte eine hohe fetomaternale Eicosapentaensäure(EPA)-PC-Ratio direkt mit dem BPD-Schweregrad (Tab. FVo8).

Schlussfolgerung. ARA-PC ist im fetalen Plasma während der gesamten Schwangerschaft gegenüber mütterlichen Werten erhöht, während DHAPC erst nach 33 w PMA mütterliche Werte übersteigt. Die Korrelation von hohem DHA-PC, niedriger ARA-/DHA-PC-Ratio und hohem EPA-PC vor 28 w PMA mit der Entwicklung einer BPD akzentuiert die Bedeutung einer PMA-adaptierten Fettsäureversorgung, impliziert Untersuchungen der Phospholipide im Nabelschnurblut zur BPD-Prädiktion und stellt Konzepte der isolierten DHA-Substitution bei extrem unreifen Frühgeborenen in Frage.

\section{FV09}

\section{Surfactantgabe unter CPAP - eine neue Applikationsmethode}

\section{Maiwald', P. Neuberger ${ }^{2}$, M. Vochem², C. Poets'}

'Eberhard-Karls-Universität Universitätsklinik für Kinderheilkunde und Jugendmedizin, Neonatologie, Tübingen, Deutschland, ${ }^{2}$ Olgahospital Stuttgart, Päd. Zentrum, Neonatologie, Stuttgart, Deutschland

Hintergrund. Bei der Behandlung von Frühgeborenen mit Atemnotsyndrom zeigt sich ein Trend, die Surfactantapplikation mittels trachealer Sonde unter CPAP-unterstützter Spontanatmung (LISA, „less invasive surfactant administration") der Intubationsmethode vorzuziehen. Hierzu wurden bislang verschiedene Techniken vorgestellt.

Fragestellung. Ziel dieser Arbeit war es, ein Medizinprodukt zu entwickeln, dass die Vorteile existierender Verfahren kombinieren kann, die Nachteile weitgehend ausschaltet und die Durchführung der Surfactant-Applikation insgesamt vereinfacht. 
Material und Methoden. Eine von Klinikern neu entwickelte, oral anwendbare Applikationshilfe (QuickSF) wurde zunächst in mehreren Sitzungen mit Fachkräften der unterschiedlichen Bereiche verbessert und anschließend in zwei Testphasen von Kinderärzten mit unterschiedlichem neonatologischem Weiterbildungsstand am Mannequin erprobt. Als Modelpuppe wurde der Neugeborenen-Intubationstrainer der Firma Laerdal verwendet. Primärziel der Arbeit war die Prüfung der Anwendbarkeit von QuickSF, Sekundärziele waren die Dauer der Gesamtprozedur und die Zeitdauer für das Einbringen eines Katheters in den Larynx am Mannequin. Zum Vergleich wurde eine den Probanden bekannte LISA-Methode herangezogen, bei der eine zunächst nasal eingeführte Sonde mittels Magillzange in die Trachea vorgeschoben wird. Abschließend wurden die Probanden zu Vor- und Nachteilen der neuen Methode befragt.

Ergebnisse. Bei Anwendung der Applikationshilfe QuickSF am Mannequin kam es in der abschließenden Erprobungsphase bei 27 Messungen zu keiner Fehlplatzierung der Trachealsonde. Die neue Methode verkürzt die Dauer der Gesamtprozedur von (MW \pm SD) 29,7 $\pm 6,7 \mathrm{~s}$ auf $11,0 \pm 4,3 \mathrm{~s}(\mathrm{p}<0,001)$ und die Dauer der Larynxeinstellung von $20,5 \pm 6,4$ auf $10,8 \pm 4,0$ s $(\mathrm{p}<0,001)$. In der Befragung wurden die positiven Aspekte der neuen Methode gegenüber der herkömmlichen LISA-Methode bestätigt. Schlussfolgerung. Die Applikationshilfe QuickSF zeigte im Mannequin-Test einen hochsignifikanten zeitlichen Vorteil gegenüber der vergleichbaren Methode mittels Magillzange und könnte eine erhebliche Verringerung der Belastung des Frühgeborenen während der LISA-SF-Gabe bedeuten. Die Handhabung war, unabhängig von der Intubationserfahrung der Testperson, insgesamt einfach und schnell zu erlernen. Ob sich diese Vorteile auch bei Anwendung am Patienten zeigen, muss in einer klinischen Studie untersucht werden.

\section{FV10}

\section{Zilienschlag unter Coffeintherapie}

\section{Bodenbeck', N. Loges', H. Omran', E. Rieger-Fackeldey²}

'Universitätsklinikum Münster, Klinik für Allg. Pädiatrie, Münster, Deutschland, ${ }^{2}$ Universitätsklinikum Münster, Klinik für Allg. Pädiatrie, Neonatologie und Pädiatrische Intensivmedizin, Münster, Deutschland

Hintergrund und Ziel. Eine gute mukoziliäre Clearance ist bei unreifen Frühgeborenen mit respiratorischer Unterstützung wichtig. Koffein wird bei Apnoen des Frühgeborenen standardmäßig zur Therapie des ApnoeBradykardiesyndroms eingesetzt. Die Auswirkung von Koffein auf die Zilienfunktion respiratorischer Zellen sind jedoch bisher nur bei Wimperntierchen untersucht worden. Wir bestimmten Zilienschlag und -funktion vor und nach Koffeingabe an humanen respiratorischen Epithelzellen.

Material und Methoden. Nasenbürstung bei 4 gesunden Erwachsenen. Einbringung des Materials in ein RPMI-Medium, Zentrifugierung und Anzüchtung eines Zellrasens auf Kollagen-Nährboden für 2-3 Wochen. Überführung der Zellen in Ernährungs-Suspensionskultur für weitere 2-3 Wochen. Anschließende Hochgeschwindigkeits-Videomikroskopie der gewachsenen Sphäroide (Megaplus EST 310T Hochgeschwindigkeits-Videokamera, Mikroskop Olympus IMT-2, Vergrösserung des Objektivs 40x, digitale Sammelrate 125 frames/s, 640×48o Auflösung) bei physiologischer Temperatur. Die Zilienschlagfrequenz wird mit dem SAVA-System ausgewertet (Sava Version 2.06, Ammons Engeneering $\left.^{\circledR}\right)$ nach publizierten Kriterien von O'Callaghan. Statistik: ShapiroWilk-Testung auf Normalverteilung, anschließend gepaarter t-Test. Ergebnisse. Bei allen Kontrollen nahm die Zilienschlagfrequenz über die Zeit ab $(18,58[16,6-21,9] \mathrm{Hz}$ nach 30 Minuten vs. 6,09 Hz nach 2,7 h [o-8,7 Hz] (median; [range]); $\mathrm{p}=0,03)$. Nach Zugabe von Coffein $(5 \mathrm{mM})$ auf die Zellen stieg die Zilienschlagfrequenz um $21 \%$ an $(17$ vs. $21,6 \mathrm{~Hz}$; [11,1 bzw. 13,3-20,4 bzw. 23,9 Hz] (median; [range]; p<0,001), nach Zugabe von 8 mM Coffein um 16\% (16,85 vs. 20,13 Hz; [12,1 bzw. 16,4-19,9 bzw. 23,4 Hz] [median; range]; $\mathrm{p}=0$,o11). Der Anstieg erfolgte innerhalb von 10 min. Zwischen den beiden Koffeinkonzentrationen gab es keinen Unterschied in der Zilienschlagfrequenz.

Schlussfolgerung. Nach Koffeingabe stieg die Zilienschlagfrequenz aller respiratorischer Epithelzellen an. Wir spekulieren, dass eine Koffeinthe- rapie bei Frühgeborenen nicht nur eine effektive Therapie von Apnoen darstellt, sondern auch eine verbesserte mukoziliäre Clearance bewirkt.

\section{FV11}

Zilienschlag/-funktion bei kranken Neugeborenen und Frühgeborenen ab 34. SSW

\section{W. Beckmann', M. Tekaat ${ }^{1}$, H. Omran', E. Rieger-Fackeldey ${ }^{2}$}

'Westf. Wilhelms-Univ.- Kinderklinik, Klinik für Kinder- und Jugendmedizin Allgemeine Pädiatrie, Münster, Deutschland, ${ }^{2}$ Universitätsklinikum Münster, Klinik und Poliklinik für Kinder- und Jugendmedizin, -Allgemeine Pädiatrie-, Neonatologie und Pädiatrische Intensivmedizin, Münster, Deutschland

Hintergrund und Ziel. Eine gute mukoziliäre Clearance ist bei Früh- und Neugeborenen wichtig. Wir untersuchten Zilienschlag und -funktion bei kranken Neugeborenen sowie Frühgeborenen ab 34. SSW bei verschiedenen Temperaturen.

Material und Methoden. Nasenbürstung bei 8 kranken, reifen Neugeborenen sowie 20 Frühgeborenen ab 34 SSW $(33+2-39+5$ SSW $)$ während des stationären Aufenthaltes, davon 24 mit respiratorischer Anpassungsstörung, 2 mit Hypoglykämien, zwei SGA und 4 Neugeboreneninfektionen. Einbringung des Materials in RPMI-Medium und anschließende Hochgeschwindigkeits-Videomikroskopie (Olympus IMT-2 Phasen-Kontrast Mikroskop mit Redlake ES-310-Turbo Monochrom Hochgeschwindigkeits-Videokamera) Vergrößerung des Objektivs 4ox, digitale Sammelrate 125 frames/s, 640×480 Auflösung) zunächst in Raumtemperatur, dann nach Aufwärmen auf $37^{\circ} \mathrm{C}$. Die Zilienschlagfrequenz wird mit dem SAVA-System ausgewertet (Sava Version 2.06, Ammons Engineering ${ }^{\circledR}$ ) nach publizierten Kriterien von O'Callaghan. Statistik: gepaarter t-Test.

Ergebnisse. Bei allen untersuchten Kindern waren zilierte Zellen im Bürstenmaterial nachweisbar, die Zilien- und Videoqualität wurde bei 28 Patienten als gut bewertet und das Material in die Auswertung einbezogen. Die Zilienschlagfrequenz bei Raumtemperatur lag bei 4,530 $\mathrm{Hz} \pm 1,724(1,29-8,69)$ [Mittelwert + SD; Range], bei $37^{\circ} \mathrm{C}$ bei $9,426 \mathrm{~Hz}$ $\pm 2,383(4,49-14,19)$ [Mittelwert + SD; Range]. Im Durchschnitt wurde die maximale Schlagfrequenz durch Aufwärmen nach 2,1 Minuten erreicht, der Anstieg der Schlagfrequenz durch Aufwärmen beträgt $136,75 \%(3,42-796,59 \%)$ [Mittelwert; Range].

Schlussfolgerung. Die Zilienschlagfrequenz ist bei Früh- und Neugeborenen bei $37^{\circ} \mathrm{C}$ deutlich höher als in Raumtemperatur. Sie liegt allerdings bei unseren Versuchen um 25,77\% unter den von O'Callaghan 1991 publizierten Daten $(12,7 \mathrm{HZ} / 0,84)$ [Mean; Stabw]. Die Schlagfrequenz von reifen Neugeborenen und späten Frühgeborenen unterschied sich nicht.

\section{FV12}

Nebulization of Poractant alfa via a vibrating membrane nebulizer in spontaneously breathing preterm lambs with binasal continuous positive pressure ventilation

M. Hütten', E. Kuypers², D. Ophelders' ${ }^{2}$, M. Nikiforou' ${ }^{2}$, R. Jellema ${ }^{3}$, H. Niemarkt ${ }^{3}$, C. Fuchs ${ }^{3}$, M. Tservistas ${ }^{3}$, R. Razetti ${ }^{4}$, F. Bianco ${ }^{4}$, B. Kramer ${ }^{3}$

${ }^{1}$ Neonatology, Department of Pediatric and Adolescent Medicine, University Clinic RWTH Aachen, Germany, and Department of Pediatrics, Maastricht University Medical Center, Maastricht, The Netherlands, Aachen, Deutschland, ${ }^{2}$ Maastricht University Medical Center, Department of Pediatrics, Maastricht, Niederlande, ${ }^{3}$ Research and Development Department, Pari Pharma GmbH, Munich, Germany, Starnberg, Deutschland, ${ }^{4}$ Research and Development Department, Chiesi Farmaceutici SpA, Parma, Italy, Parma, Italien

Background. Surfactant replacement therapy is the gold standard treatment of neonatal respiratory distress (RDS). Nebulization is a non-invasive mode of surfactant administration. We administered Poractant alfa (Curosurf $\left.{ }^{\circledast}\right)$ via a vibrating perforated membrane nebulizer (e-flow Neonatal Nebulizer) to spontaneously breathing preterm lambs during binasal continuous positive pressure ventilation (CPAP). We hypothesized that surfactant nebulization would improve oxygenation and lung 
function, and that these effects would be independent from treatment duration and air humidification.

Methods. 16 preterm lambs were operatively delivered at a gestational age of $133 \pm 1 \mathrm{~d}$ (term $\sim 15 \mathrm{O}$ ), and connected to CPAP applied via customized nasal prongs. Nebulization was performed (i) with saline or (ii) with surfactant for 3 hours in humidified or (iii) non-humidified air, and with surfactant (iv) for $60 \mathrm{~min}$ or (v) for $30 \mathrm{~min}$. We measured arterial oxygenation, lung gas volumes and surfactant pool size and deposition. Results. Nebulization of surfactant in humidified air compared to saline nebulization improved oxygenation after $3 \mathrm{~h}\left(\mathrm{PaO}_{2} 422 \pm 23\right.$ vs. $233 \pm 38$ $\mathrm{mmHg}, \mathrm{p}<0,05)$ and lung gas volumes $(53.8 \pm 7.0$ vs. $26.8 \pm 3.8 \mathrm{~mL} / \mathrm{kg}$ body weight at pressure of $40 \mathrm{~cm} \mathrm{H} \mathrm{H}_{2} \mathrm{O}$ ). In this group, surfactant was preferentially distributed to the lower lung lobes. Shorter nebulization times and $3 \mathrm{~h}$ nebulization in dry air resulted in a different pattern of surfactant distribution and did not increase oxygenation and lung gas volumes. Nebulized surfactant reached all lung lobes, however the increase of surfactant pool size missed statistical significance.

Conclusion. In combination with a customized nebulizer, Poractant alfa was distributed to the lung of spontaneously breathing animals and improved physiological parameters. However, positive treatment effects of surfactant nebulization depended on surfactant dose, treatment duration, air humidity and local surfactant distribution. Factors influencing surfactant distribution and treatment effects need to be identified in detail to allow clinical application.

\section{FV13}

\section{Moderate prenatal cigarette smoke predisposes for postnatal lung injury in neonatal mice}

N. Kamgari', P. Oak', T. Gimm', G. John-Schuster' ${ }^{2}$, A. Yildirim², A. Hilgendorff ${ }^{3}$ ${ }^{1}$ Comprehensive Pneumology Center (CPC), München, Deutschland, ${ }^{2}$ Comprehensive Pneumology Center (CPC), Neuherberg, Deutschland, ${ }^{3} \mathrm{Pe}-$ rinatalzentrum Grosshadern, Neonatologische Intensivstation, München, Deutschland

Introduction. Chronic lung disease (CLD) in the preterm infant is characterized by impaired lung growth, extracellular matrix (ECM) remodeling and dysregulated growth factor signaling. Clinical studies show association of prenatal cigarette smoke (pCS) with premature birth and low birth weight, risk factors for the development of the disease. Whether moderate $\mathrm{pCS}$, not inducing fetal growth restriction, affects pulmonary development or the susceptibility to lung injury is largely unknown. We therefore studied the role of pCS on pulmonary development and response to injury by postnatal mechanical ventilation with oxygen-rich gas $\left(\mathrm{MV}-\mathrm{O}_{2}\right)$ in newborn mice.

Methods. C57BL/6 (WT) mice were exposed to moderate pCS (50omg/ $\mathrm{m} 3,50 \mathrm{~min} /$ day) or filter air (FA) from day 7 to 18 of pregnancy. 5 to 7 day old newborn mice from both groups received $\mathrm{MV}-\mathrm{O}_{2}\left(\mathrm{FiO}_{2}=0.4\right)$ or breathed room air (control) for 8 hours. Lungs were collected for histological analysis (alveolar area, radial alveolar count, septal density), assessment of ECM structure and micro-vessel number.

Results. Unventilated neonatal mice exposed to pCS exhibited no significant differences with respect to alveolar number or size when compared to FA control pups. In both groups (pCS, FA), MV-O2 led to the development of larger and fewer alveoli indicating impaired alveolar development in these lungs. However, the lungs of unventilated neonatal pCS mice exhibited disordered elastin deposition in contrast to FA control pups, comparable to changes post- MV-O2. Furthermore, $8 \mathrm{~h} \mathrm{MV-O} 2$ caused a 2.3 -fold decrease in the number of micro-vessel $(20-100 \mu \mathrm{m})$ in the lungs of neonatal mice exposed to pCS when compared to ventilated newborn mice from the FA group.

Conclusion. Moderate pCS could predispose to the development of CLD in the immature lung when exposed to postnatal MV-O2 as indicated by impaired micro-vessel formation and altered elastin deposition in these lungs. Future studies need to establish the relevance of these changes in CLD pathophysiology and their potential for therapeutic approaches.

\section{Neonatologie: Randomisierte Studien}

\section{FV14}

Einfluss einer höheren Eiweißzufuhr auf das Wachstum von sehr unreifen Frühgeborenen. Erste Ergebnisse einer prospektiven, randomisiert-kontrollierten Interventionsstudie

\section{Maas', M. Mathes', C. Bleeker', J. Bürkle', C. Wiechers', C. Poets', A. Franz ${ }^{1}$}

'Eberhard-Karls-Universität Universitätsklinik für Kinderheilkunde und Jugendmedizin, Neonatologie, Tübingen, Deutschland

Hintergrund und Fragestellung. Eine Erhöhung der Eiweißzufuhr führt bei Frühgeborenen (FG) zu einer verbesserten Stickstoffbilanz. Zur Frage einer Wachstumsbeschleunigung durch eine höhere Eiweißzufuhr $(>3,5 \mathrm{~g} / \mathrm{kg} / \mathrm{d})$ sind die Ergebnisse prospektiver, randomisierter Studien gegenwärtig widersprüchlich. Primäres Ziel dieser Untersuchung war zu prüfen, ob eine höhere, den ESPGHAN-Empfehlungen entsprechende enterale Eiweißzufuhr zu einer Verbesserung der Gewichtszunahme bei überwiegend muttermilchernährten FG führt.

Patienten und Methoden. Prospektive, monozentrische, randomisiertkontrollierte und partiell geblindete Interventionsstudie mit drei Parallelgruppen. Eingeschlossen wurden FG mit einem Gestationsalter (GA) bei Geburt $<32$ SSW und einem Geburtsgewicht (GG) <1500 g. Folgende, a priori hierarchisch geordneten Hypothesen sollten geprüft werden: Hypothese A (übergeordnete Hypothese): Eine an den Empfehlungen der ESPGHAN orientierte Eiweißzufuhr von ca. 4,5 g/kg/d führt im Vergleich zu einer Standardernährung mit ca. 3,5-4,o g/kg/d Eiweiß zu einer Verbesserung des Wachstums der FG. Hypothese B: Eine individualisierte, am tatsächlichen Eiweiß- und Energiegehalt der Frauenmilch ausgerichtete Supplementierung führt im Vergleich zur standardisierten Verwendung eines neuen, proteinreicheren Frauenmilchsupplements bei ähnlicher Eiweißzufuhr zu einer Verbesserung des Wachstums. Berichtet werden Ergebnisse zu Hypothese A. Primäres Zielkriterium war eine Verbesserung der täglichen Gewichtszunahme zwischen Geburt und Interventionsende um 3 g/kg/d. Statistische Signifikanz war definiert als $p<0,05$. Deskriptive Daten werden als Median (p25/p75) angegeben.

Ergebnisse. Untersucht wurden 6o Frühgeborene. Die tägliche Gewichtszunahme von Geburt bis Interventionsende unterschied sich zwischen Standard- $(\mathrm{n}=30)$ und Hocheiweißgruppe $(\mathrm{n}=30)$ nicht statistisch signifikant $[16,0$ (14,5/18,o) g/kg/d vs. 15,6 (14,3/17,5) g/kg/d; p=0,71]. Dies traf auch auf die Interventionsperiode zu [Gewichtszunahme Standardgruppe 18,o $(16,3 / 20,1) \mathrm{g} / \mathrm{kg} / \mathrm{d}$, Hocheiweißgruppe $18,3(16,6 / 19,7) \mathrm{g} / \mathrm{kg} / \mathrm{d} ; \mathrm{p}=0,93]$. GG und GA waren in beiden Behandlungsgruppen ähnlich [1215 (1065/1393) g vs. $1193(984 / 1326) \mathrm{g} ; \mathrm{p}=0,60$ und 30,0 (29,0/31,1) SSW vs. $29,7(27,9 / 31,0)$ SSW; $\mathrm{p}=0,19]$, wie auch die Länge der Interventionsperiode [43 (32/51) d vs. $40(30 / 58) \mathrm{d} ; \mathrm{p}=\mathrm{o}, 89)$ und die Dauer des stationären Aufenthalts [52 (42/65) d vs. 52 (37/70) d; $\mathrm{p}=0,59$ ].

Schlussfolgerung. Die Erhöhung der Eiweißzufuhr gemäß ESPGHANEmpfehlung führte in dieser Studie nicht zu einer Verbesserung der Gewichtszunahme. Allerdings lag die postnatale Gewichtsentwicklung in beiden Gruppen annähernd im Bereich intrauteriner Wachstumskurven.

\section{Pädiatrische Intensivmedizin: Schädel-Hirn-Trauma}

\section{FV15}

Anwendbarkeit der „Termination of resuscitation“(TOR)Kriterien auf präklinische Kinderreanimationen in Aachen

V. Rotering', M. Schoberer', S. Trepels-Kottek', K. Heimann', T. Orlikowsky' 'Uniklinikum der RWTH Aachen, Klinik für Kinder- und Jugendmedizin, Sektion Neonatologie, Aachen, Deutschland

Hintergrund. Präklinische Reanimationen sind im Kindes-, und Erwachsenenalter mit schlechtem Überleben assoziiert (5-10\%). Die große emotionale Betroffenheit des Rettungsdienstpersonals, sowie die höhere Lebenserwartung im Falle eines Überlebens, führen bei Kindern tendenziell zu einer „Übertherapie“ kardiozirkulatorischer Arreste. Die frühzeitige Identifikation aussichtsloser Fälle anhand zuverlässiger Prädiktoren soll 
dies vermeiden und Betroffene, wie Angehörige, vor einer medizinisch ungerechtfertigten Traumatisierung bewahren. Solche Prädiktoren müssen für die Detektion der tatsächlich hoffnungslosen Fälle 100\%ig spezifisch sein bei gleichzeitig möglichst großer Sensitivität. Morrison und Verbeek haben für das Erwachsenenalter TOR-Kriterien für eine frühzeitige Einstellung des Basic- (BLS) und Advanced Life Support (ALS) entwickelt. Vergleichbare Kriterien existieren für das Kindesalter nicht.

Fragestellung. An einem Kollektiv von Patienten bis 18 Jahren, die nach präklinischer Reanimation im UK Aachen behandelt wurden, wird die Übertragbarkeit der der ALS-TOR-Kriterien nach Morrison auf das Kindesalter retrospektiv untersucht.

Methoden. Von 75 präklinischen Reanimationsfällen unserer Einrichtung vom 1.01.2003 bis 1.01.2014 (s. a. eingereichtes Abstract „Epidemiologie und Outcome präklinischer Reanimationen bei Kindern und Jugendlichen in Aachen 2003-2013") wurden diejenigen mit gesichert kardiozirkulatorischem Arrest bei Rettungsdienst-Eintreffen ausgewählt $(n=43)$. Die prädiktive Validität der TOR-Kriterien für das Versterben der Kinder wurde untersucht. Das Vorliegen mind. einer der 5 folgenden Prädiktoren für ein günstiges Outcome wird dazu untersucht: Bezeugung des Arrests durch Rettungsdienstpersonal oder Laien, Z. n. Laienreanimation, Z. n. Defibrillation, Spontanzirkulation (ROSC) vor Transport. Nur bei Abwesenheit all dieser Prädiktoren darf die Reanimation vorzeitig eingestellt werden.

Ergebnisse. Überlebende Patienten: 14 (32,6\%),verstorbene Patienten: 29 $(67,4 \%)$. Für die Vorhersage der fehlenden Aussicht auf Überleben (keines von 5 Kriterien erfüllt, TOR möglich) ergibt sich:

- Erfüllt bei 4 Patienten (9,3\%)

- Sensitivität: 4/29 verstorbenen Patienten (13,8\%)

- Spezifität: 14/14 überlebenden Patienten (100\%)

- Positiver Vorhersagewert für Versterben: 4/4 Patienten (100\%)

- Negativer Vorhersagewert für Versterben: 14/39 Patienten (35,9\%)

Diskussion. Bei retrospektiver Anwendung auf unser Patientenkollektiv haben die TOR-Kriterien die zentrale Forderung nach 100\%iger Spezifität und 100\%iger positiver Vorhersagewahrscheinlichkeit erfüllt. Auffällig ist die geringe Sensitivität der TOR-Regel. Nur bei einer geringen Patientenzahl hätte sie Anwendung gefunden. Dies entspricht nicht den Erfahrungen bei Erwachsenen, bei denen Reanimationen in 35-50\% der Fälle aufgrund der TOR-Kriterien beendet werden können. Am ehesten ist dies auf die im Kindesalter überwiegend nichtkardialen Ursachen der Kreislaufstillstände zurückzuführen. Vor dem Hintergrund der geringen Sensitivität ist eine allgemeine Anwendung der Regel bei Kindern nicht zu empfehlen, insbesondere da allgemeingültige Aussagen zur Spezifität Überprüfungen an deutlich größeren Kollektiven und weitere prospektive Studien erfordern. Wir erwarten allerdings, dass die Erweiterung der Kriterien, etwa um laborchemische Messgrößen, wie $\mathrm{pH}$ oder Blutzucker die Sensitivität deutlich erhöhen würde.

\section{Zerebrale Oxygenierung und aEEG}

\section{FV16 \\ Kann ein in den ersten 72 Lebensstunden abgeleitetes aEEG eine Prognose bezüglich Entwicklungsstörungen bei Frühgeborenen geben?}

H. Müller', N. Bruns', B. Hüning', T. Storbeck', F. Dransfeld', I. Mund', A. Neuhausen', C. Weiß², U. Felderhoff-Müser ${ }^{1}$

'Universitäts-Kinderklinik Essen, Klinik für Kinderheilkunde I/Perinatalzentrum, Essen, Deutschland, ${ }^{2}$ Universitätsklinikum Mannheim, Abteilung für Medizinische Statistik/Biomathematik, Mannheim, Deutschland

Hintergrund. Das aEEG bietet die Möglichkeit, Früh- und Neugeborene auf der neonatologischen Intensivstation neurologisch zu untersuchen. Dadurch kann sowohl die Reifung des Gehirns beobachtet als auch Krampfpotentiale erkannt werden.
Fragestellung. Ziel ist es zu untersuchen, ob das aEEG frühzeitig prognostische Aussagen hinsichtlich der neurologischen Entwicklung der untersuchten Frühgeborenen zulässt.

Patienten und Methoden. Es wurden 59 Kinder aus den Geburtsjahrgängen 2009-2012 untersucht. Bei diesen Kindern wurde in den ersten 72 Lebensstunden ein aEEG durchgeführt, welches von 2 Personen nach dem Burdjalov-Score und nach Hellström-Westas ausgewertet wurde. Während des stationären Aufenthalts wurden regelmäßig Sonografien des Gehirns sowie ein konventionelles EEG vor Entlassung durchgeführt. Im korrigierten Alter von 24 Monaten erfolgte die Bayley-II-Testung im Rahmen einer Vorstellung im sozialpädiatrischen Zentrum, wobei ein schlechtes neurologisches Outcome mit einem MDI- und/oder PDI-Score von kleiner 70 definiert wurde. Zudem wurden sämtliche perinatalen Faktoren sowie klinischen Parameter bei den Patienten erfasst.

Ergebnisse. Die 59 untersuchten Kinder zeigten ein mittleres Geburtsgewicht von $1153 \mathrm{~g}$ (450-3480 g) und ein mittleres Gestationsalter von 27,8 vollendeten SSW (23-38 SSW). Das Outcome (gut/schlecht) im korrigierten Alter von 24 Monaten korrelierte nicht mit den beiden aEEG-Scores. Krampfpotentiale (Hellström-Westas-Score) in den ersten 24 Lebensstunden waren signifikant mit der maximalen Ausprägung einer intraventrikulären Blutung $(\mathrm{p}<0,01)$ und dem Auftreten eines Hydrocephalus $(\mathrm{p}<0,05)$ assoziiert. Zudem zeigte sich ein signifikanter Zusammenhang zwischen Krampfpotentialen in den Lebensstunden 25-48 und einem pathologischen EEG vor Entlassung ( $\mathrm{p}<0,05)$ sowie zwischen den Krampfpotentialen in den Lebensstunden 49-72 und dem Auftreten von klinischen Krämpfen während des stationären Aufenthalts ( $\mathrm{p}<0,05)$.

Schlussfolgerung. Die erfassten aEEG-Parameter ließen keine Aussage auf das neurologische Outcome im korrigierten Alter von 24 Monaten zu. Allerdings kann eine Analyse des aEEGs auf Krampfpotentiale nach Hellström-Westas-Hinweise auf das Auftreten einer intraventrikulären Blutung sowie auf die Krampfaktivität, die sich auch in einer entsprechenden Klinik bzw. in einem pathologischen EEG vor Entlassung äußert, geben.

\section{FV17}

Nichtinvasive intraoperative Messung von cerebraler Sauerstoffsättigung, Mikroperfusion und Sauerstoffmetabolismus bei Säuglingen

A.Müller', F.Neunhoeffer', M.Hofbeck', F.Fideler', C.Schwarz', M.Kumpf', J.Fuchs ${ }^{4}$ 'Eberhard-Karls-Universität Universitätsklinik für Kinderheilkunde und Jugendmedizin, Kinderkardiologie, Pulmologie und pädiatrische Intensivmedizin, Tübingen, Deutschland, ${ }^{2}$ Universitätsklinikum Tübingen, Anästhesie und Intensivmedizin, Tübingen, Deutschland, ${ }^{3}$ Eberhard-Karls-Universität Universitätsklinik für Kinderheilkunde und Jugendmedizin, Neonatologie, Tübingen, Deutschland, ${ }^{4}$ Universitätsklinikum Tübingen, Klinik für Kinderund Jugendmedizin, Abteilung für Kinderchirurgie, Tübingen, Deutschland

Hintergrund. Momentan gibt es kein standardisiertes intraoperatives Monitoring von cerebraler Sauerstoffsättigung und Mikroperfusion bei Säuglingen. Ziel der Studie war die nicht-invasive Messung der cerebralen Sauerstoffsättigung, der Mikroperfusion und des Sauerstoffmetabolismus bei Säuglingen während operativer Eingriffe.

Methoden. Bei 21 Säuglingen wurden während abdominellen Eingriffen mit Hilfe einer Kombination von Laserdoppler- und Gewebsspektrometrie in Echtzeit die relative cerebrale Sauerstoffsättigung $\left(\mathrm{rCSO}_{2}\right)$, die relative cerebrale Hämoglobinmenge $(\mathrm{rcHb})$ und der relative cerebrale Blutfluss (rCFB) gemessen. Mittels arterieller Sauerstoffsättigung (aSO2) konnte die fraktionelle cerebrale Sauerstoffextraktion (cFTOE) und der angenäherte cerebrale Sauerstoffumsatz $(\mathrm{aCMRO} 2)$ berechnet werden. Das Einverständnis der Eltern wurde eingeholt und die Studie wurde von der zuständigen Ethikkommission genehmigt.

Ergebnisse. Insgesamt wurden 21 Kinder untersucht. Im Median waren die Kinder 41 Tage alt (Range: 1-172 Tage) und 4100 g schwer (Range: $1600-7600$ g). 15 Kinder waren männlichen, 6 Kinder weiblichen Geschlechtes. Die aSO2, das $\mathrm{CO}_{2}$, der $\mathrm{pH}$ und der $\mathrm{Hb}$ zeigten perioperativ keine signifikanten Veränderungen. $\mathrm{Die} \mathrm{CSO}_{2}$ stieg intraoperativ von 
$65.02 \%(51,47-85,76 \%)$ auf $67,062 \%(52,74-79,14 \%)$ an und lag postoperativ bei $69,87 \%(51,74-80,65 \%)$. Das crHb stieg von präoperativ 67,17 AU (48,96-105,65 AU) auf 69,83 AU (49,72-105,32 AU) intraoperativ an und postoperativ auf 70,30 AU (40,79-105,82 AU). Die cFTOE nahm im Vergleich zu präoperativ sowohl intraoperativ als auch postoperativ von $0,35(0,05-0,46)$ präoperativ auf $0,311(0,16-0,46)$ intraoperativ und o,29 (o,19-0,43) postoperativ ab. Die aCMRO2 stieg signifikant von 4,53 $\mathrm{AU}(0,86-7,16 \mathrm{AU})$ präoperativ auf 5,12 $\mathrm{AU}(2,62-9,98 \mathrm{AU})$ intraoperativ und 4,91 AU (2,99-12,07 AU) postoperativ an $(\mathrm{p}<0,05)$. Der cerebrale Blutfluss zeigte ebenfalls einen signifikanten Anstieg von präoperativ 422,92 AU (291,11-714,17 AU) auf 484,77 AU (317,87-1270,64 AU) intraoperativ und auf 511,14 AU (360,93-929,65 AU) postoperativ ( $\mathrm{p}<0,05)$. Eine Abnahme der cerebralen Sauerstoffsättigung um mehr als 20\% im Vergleich zur präoperativen Baseline bezogen auf die Dauer der Operation betrug $0,00 \% \mathrm{~min} /$ Stunde OP $(0,00-4,03)$.

Schlussfolgerung. Die cerebrale Sauerstoffsättigung und Mikroperfusion wurde intraoperativ im Mittel nicht signifikant beeinträchtigt. Dennoch konnte gezeigt werden, dass es in Einzelfällen intraoperativ zu Entsättigungen kommen kann. Die Kombination von Laserdoppler und Gewebsspektrometrie bietet die Möglichkeit eines nicht-invasiven Monitorings nicht nur der cerebralen Sauerstoffsättigung sondern des gesamten Sauerstoffmetabolismus. Dies könnte eine adäquatere intraoperative cerebrale Überwachung ermöglichen.

\section{FV18}

Correlation between NIRS and aEEG in hypothermia treated newborns with hypoxic-ischemic encephalopathy

K. Goeral', B. Urlesberger', V. Giordano', L. Schmidt', M. Weninger', A. Berger', K. Klebermaß-Schrehof', M. Olischar'

'Univ. Klinik für Kinder und Jugendheilkunde, Abt. für Neonatologie, Pädiatrische Intensivmedizin und Neuropädiatrie, Wien, Österreich, ${ }^{2}$ Univ. Klinik für Kinder- und Jugendheilkunde, Abteilung für Neonatologie, Graz, Österreich

Background. Hypoxic-ischemic encephalopathy (HIE) still is a major cause of neonatal mortality and morbidity. The amplitude-integrated electroencephalogram (aEEG) is reliable for prediction of outcome in asphyxiated neonates. Near-infrared spectroscopy (NIRS) offers the possibility to continuously monitor cerebral oxygen saturation. Only few data have been published on the combined use of NIRS and aEEG in neonates cooled for HIE. The aim of this study was to investigate the correlation between continuous aEEG and NIRS during the first days of life in HIE. Methods. aEEG: Brain function was measured continuously immediately after the hypoxic event, during hypothermia, until the end of rewarming using a Olympic 6000 (Natus( ). aEEG tracings were analysed for background patterns, appearance of sleep-wake cycling and the presence of seizure activity. Furthermore, a combined aEEG-score was calculated for the timeperiod before, during and after hypothermia. NIRS: Patients were monitored using NIRS (INVOS System by Covidien $\odot$ ) for the mentioned time period. Changes in regional cerebral oxygen saturation $\left(\mathrm{rcSO}_{2}\right)$ were measured. Fractional tissue oxygen extraction (FTOE) was calculated for the entire duration of the measurement in order to investigate the balance between oxygen delivery and oxygen supply.

Results. A significant correlation coefficient (CorrC) was found between $\mathrm{SWZ}$ and $\mathrm{rcSO}_{2}$ post cooling (CorrC: 0.78 ) as well as FTOE during (CorrC: 0.69) and post cooling (CorrC: 0.95). Furthermore, a significant CorrC was found between the total aEEG score and $\mathrm{rcSO}_{2}$ post cooling (CorrC: 0.58 ) as well as FTOE during (CorrC: 0.61) and post cooling (CorrC: 0.79).

Conclusion. Our data show a significant correlation between continuous aEEG monitoring, which is a valuable and reliable method for prediction of neurodevelopmental outcome in neonates with HIE, and NIRS measurements. Therefore, we suggest that NIRS can serve as an additional prognostic tool in HIE.

\section{FV19}

Referenzwerte für regionale zerebrale Oxygenierung (cTOI) bei Neugeborenen während der Adaptationsphase

N. Baik', B. Urlesberger', B. Schwaberger', G. Schmölzer², L. Mileder', A. Avian', G. Pichler ${ }^{1}$

'Universitätsklinik für Kinder- und Jugendheilkunde, Klinische Abteilung für Neonatologie, Graz, Österreich, ${ }^{2}$ Department of Neonatology, Royal Alexandra Hospital, Edmonton, Canada, Edmonton, Kanada, ${ }^{3}$ Medizinische Universität Graz, Medizinische Informatik und Statistik, Graz, Österreich

Hintergrund. Nichtinvasive Überwachung des Gehirns mit Nahinfrarot-Spektroskopie (NIRS) während der Adaptationsphase nach der Geburt ist von wachsendem Interesse. Die Referenzwerte von $\mathrm{CrSO}_{2}$ und cFTOE gemessen mit INVOS $5100 \mathrm{C}$ wurden daher bereits etabliert.

Fragestellung. Das Ziel dieser prospektiven Beobachtungsstudie war es die Referenzwerte und Perzentilen der regionalen zerebralen Oxygenierung (cTOI) und der zerebralen Sauerstoffgewebsextraktion (cFTOE) gemessen mit NIRO 200NX - während der ersten 15 Minuten nach der Geburt bei Früh- und Neugeborenen mit unauffälliger Adaptationsphase zu definieren.

Material und Methoden. cTOI wurde mit NIRO $200 \mathrm{NX}$ in den ersten 15 Minuten nach der Kaiserschnittentbindung in Früh- und Neugeborenen gemessen. Der NIRS Sensor wurde an der Stirn rechts frontal angelegt. Periphere arterielle Sauerstoffsättigung $\left(\mathrm{SpO}_{2}\right)$ und die Herzfrequenz (HF) wurden kontinuierlich mittels Pulsoxymetrie gemessen. cFTOE wurde aus cTOI und $\mathrm{SpO}_{2}$ berechnet. Neugeborene, die während der Adaptationsphase Sauerstoff- und/oder respiratorische Unterstützung erhielten, wurden ausgeschlossen.

Ergebnisse. Insgesamt wurden 230 Neugeborene eingeschlossen: 82 Neugeborene mussten aufgrund einer Sauerstoff- und/oder respiratorische Unterstützung ausgeschlossen werden. Die Daten der $148 \mathrm{Neu}-$ geborenen (140 Reifgeborene/8 Frühgeborene) wurden analysiert, um Referenzwerte und Perzentilen zu definieren. 50. Perzentile (10.-90. Perzentile ) von cTOI betrug $55 \%(38-75)$ in $2 \mathrm{~min}, 65 \%(50-78)$ in $5 \mathrm{~min}$, $74 \%(61-85)$ in $10 \mathrm{~min}$, und $74 \%(61-84)$ in 15 min nach der Geburt. 50. Perzentile (10. bis 9o. Perzentile ) von cFTOE betrug 0,24 (0,11-0,44) bei $2 \mathrm{~min}, 0,20(0,10-0,35)$ in $5 \mathrm{~min}, 0,21(0,09-0,35)$ bei $10 \mathrm{~min}$ und 0,24 $(0,13-0,37)$ bei 15 min nach der Geburt.

Schlussfolgerung. Mit der vorliegenden Beobachtungsstudie werden erstmals Referenzbereiche und Perzentilen von cTOI und cFTOE gemessen mit NIRO 200NX definiert. Diese Perzentilen sind Grundlage für zukünftige klinische Anwendung bei Verwendung des NIRO 200NX.

\section{FV20}

Veränderungen des zerebralen Blutvolumens in der Adaptationsphase von Früh- und Reifgeborenen mit und ohne Atemunterstützung

B. Schwaberger', G. Pichler', N. Baik', C. Binder-Heschl', B. Urlesberger'

'Medizinische Universität Graz, Klinische Abteilung für Neonatologie, Universitätsklinik für Kinder- und Jugendheilkunde, Graz, Österreich

Hintergrund. Nahinfrarotspektroskopie (NIRS) ermöglicht eine kontinuierliche, nichtinvasive Messung der zerebralen Konzentrationsänderungen von oxygeniertem $\left(\Delta \mathrm{O}_{2} \mathrm{Hb}\right)$ und desoxygeniertem Hämoglobin $(\Delta \mathrm{HHb})$ bei Neugeborenen. Veränderungen des totalen Hämoglobins $\left(\Delta \mathrm{cHb}=\Delta \mathrm{O}_{2} \mathrm{Hb}+\Delta \mathrm{HHb}\right)$ können unter Berücksichtigung der Hämoglobinkonzentration in den großen Gefäßen in Veränderungen des zerebralen Blutvolumens $(\triangle \mathrm{CBV})$ umgerechnet werden. Unsere Studiengruppe zeigte einen signifikanten Abfall des CBV bei gesunden Reifgeborenen in den ersten 15 Lebensminuten. Bislang gibt es jedoch keine Daten, wie sich das CBV während der postnatalen Adaptationsphase von Früh- und Reifgeborenen mit und ohne Atemunterstützung verhält.

Fragestellung. Wie verändert sich das $\triangle \mathrm{CBV}$ in der Adaptationsphase von Früh- und Reifgeborenen mit und ohne Atemunterstützung?

Material und Methode. Diese Sin gle-Center-Studie wurde als prospektive Beobachtungsstudie durchgeführt. Inkludiert wurden Früh- und 
Reifgeborene mit und ohne Notwendigkeit einer Atemunterstützung nach Schnittentbindung. Für die NIRS-Messungen wurde ein „NIRO200-NX“ (Hamamatsu; Japan) verwendet. Der NIRS-Sensor wurde rechts frontal mit elastischer Binde fixiert. Der Verlauf des $\triangle \mathrm{CBV}$ wurde für die unmittelbare postnatale Adaptationsphase über 15 Minuten berechnet.

Ergebnisse. Es wurden insgesamt 204 Neugeborene inkludiert, davon waren 45 Frühgeborene ( 37 mit und 8 ohne Atemunterstützung) mit einem mittleren Gestationsalter von $33 \pm 2$ Wochen und 159 Reifgeborene (19 mit und 140 ohne Atemunterstützung) mit einem mittleren Gestationsalter von $39 \pm 1$ Wochen. $\triangle \mathrm{CBV}$ Bei Früh- und Reifgeborenen zeigte sich ein signifikanter Abfall des CBV in den ersten 15 Lebensminuten. Bei Frühgeborenen ohne Atemunterstützung ist das CBV um o,9 $( \pm 1,2)$ $\mathrm{ml} / 100 \mathrm{~g}$ Gehirngewebe, bei Frühgeborenen mit Atemunterstützung um $0,4( \pm 1,3) \mathrm{ml} / 100 \mathrm{~g}$ Gehirngewebe abgefallen. Bei Reifgeborenen ohne Atemunterstützung ist das CBV um 1,2 $( \pm 2,2) \mathrm{ml} / 100$ g Gehirngewebe, bei Reifgeborenen mit Atemunterstützung um o,8 $( \pm 1,1) \mathrm{ml} / 100 \mathrm{~g}$ Gehirngewebe abgefallen.

Schlussfolgerung. Bei Früh- und Reifgeborenen findet man unmittelbar postnatal einen Abfall des CBV, wobei dieser bei gesunden Reifgeborenen ohne Atemunterstützung am stärksten ausgeprägt ist. Inwieweit diese Beobachtung vor allem bei Frühgeborenen mit Risiko einer zerebralen Schädigung von klinischer Relevanz ist, muss in zukünftigen Studien geklärt werden.

\section{FV21 \\ Comparison of two different scoring systems for amplitude- integrated EEG in premature infants and correlation with the neurodevelopmental outcome}

L. Schmidt', K. Göral', V. Giordano', E. Obwegeser', C. Czaba', M. Olischar', M. Weninger', A. Berger', K. Klebermass-Schrehof

'Universitätsklinik für Kinder- und Jugendheilkunde; Medizinische Universität Wien, Abteilung für Neonatologie, pädiatrische Intensivmedizin und Neuropädiatrie, Wien, Österreich

Introduction. The amplitude-integrated EEG (aEEG) is becoming more important to monitor brain activity in premature infants. Studies have shown that early aEEGs correlate with later neurodevelopmental outcome. Various scoring systems for the assessment of aEEGs are being used, which complicates the comparison of published data. The aim of our study was to compare two scoring systems for aEEG and correlate with the neurodevelopmental outcome.

Methods. 114 preterm infants [gestational age (GA) $<30$ weeks of gestation] who had an aEEG within the first 14 days of life and a neurodevelopmental outcome at the age of 2 were retrospectively included into the study. The aEEGs had been analyzed with the aEEG sum score [background activity $(\mathrm{BA})=$ percentage of different background patterns based on gestational age related standard values, the occurrence of sleep-wake cycles (SWC) and seizure activity] and the Burdjalov score [continuity (Co), cyclicity (Cy), amplitude of lower border (LB) and bandwidth span and amplitude of lower border (B) of the aEEGs - regardless of gestational age] and the results had been correlated with the outcome [Bayley Scales (MDI and PDI) at the age of 2].

Results. Both total scores $(\mathrm{p} \leq \mathrm{0.0001})$ and the different subscores correlated statistically significant ( $\mathrm{p} \leq \mathrm{0.0001}$ ) with each other. Both total scores showed significant correlation (Burdjalov score $\mathrm{p}=0,003$ and aEEG sum score $\mathrm{p} \leq \mathrm{o} .0001$ ) with the outcome. The Burdjalov score showed increasing values with increasing GA, while the aEEG sum score showed no differences in the various GA.

Conclusion. Both scoring systems allow a simple classification of aEEGs. The Burdjalov score shows specific differences for the GA, while the aEEG sum score correlates better with the later outcome.

\section{Neonatologie: Zerebrale Oxygenierung}

\section{FV22}

Blutdruck während der Adaptationsphase: Ist der mittlere arterielle Blutdruck (MAD) relevant für die regionale zerebrale Oxygenierung (crSO2)?

N. Baik', B. Urlesberger', B. Schwaberger', G. Schmölzer', A. Avian', G. Pichler' 'Universitätsklinik für Kinder- und Jugendheilkunde, Klinische Abteilung für Neonatologie, Graz, Österreich, ${ }^{2}$ Department of Neonatology, Royal Alexandra Hospital, Edmonton, Canada, Edmonton, Kanada, ${ }^{3}$ Medizinische Universität Graz, Medizinische Informatik und Statistik, Graz, Österreich

Fragestellung. Ziel dieser Studie war es, einen möglichen Zusammenhang zwischen regionaler zerebraler Oxygenierung ( $\left.\mathrm{crSO}_{2}\right)$ und mittlerem arteriellen Druck (MAD) bei Früh- und Reifgeborenen während der Adaptationsphase zu untersuchen.

Material und Methode. In diese prospektive Beobachtungsstudie wurden Früh- und Reifgeborene eingeschlossen. Die zerebrale regionale Oxygenierung ( $\mathrm{crSO}_{2}$ ) wurde mittels Nahinfrarot-Spektroskopie (NIRS) mit dem INVOS $5100 \mathrm{C}$ während der neonatalen Adaptationsphase (15 Minuten unmittelbar nach der Geburt) gemessen. Der NIRS Sensor wurde an der Stirn links frontal angelegt. Ferner wurde ein Pulsoxymeter angelegt, um die arterielle Sauerstoffsättigung $\left(\mathrm{SpO}_{2}\right)$ und die Herzfrequenz (HF) zu überwachen. In der 15. Lebensminute wurde einmalig der Blutdruck am linken Oberarm gemessen. Die zerebrale Sauerstoffgewebsextraktion (cFTOE) wurde aus $\mathrm{SpO}_{2}$ und $\mathrm{crSO}_{2}$ berechnet. Um den Zusammenhang zwischen cFTOE und MAD zu untersuchen, wurde eine Korrelationsanalyse durchgeführt.

Ergebnisse. Es wurden insgesamt 462 Früh- $(\mathrm{n}=186)$ und Reifgeborene $(n=276)$ eingeschlossen. Das mittlere Gestationsalter betrug $31,0 \pm 3,5$ Wochen bei Frühgeborenen, 38,9 $\pm 0,8$ Wochen bei Reifgeborenen, das mittlere Geburtsgewicht 1591 \pm 630 g bei Frühgeborenen, 3331 $\pm 461 \mathrm{~g}$ bei Reifgeborenen. Die Korrelationsanalyse zeigte keinen statistisch signifikanten Zusammenhang zwischen MAD und cFTOE bei Reifgeborenen, im Gegensatz dazu gab es aber eine statistisch signifikante negative Korrelation bei Frühgeborenen zwischen MAD und cFTOE ( $\mathrm{p}=0,02)$.

Diskussion. Die vorliegende Beobachtungsstudie zeigt einen statistisch signifikanten Zusammenhang zwischen cFTOE und MAD bei Frühgeborenen, obwohl es keinen Zusammenhang bei Reifgeborenen gibt. Dies könnte darauf hindeuten, dass bei Reifgeborenen eine funktionierende Gefäßautoregulation bereits während der Adaptationsphase existiert, während diese bei Frühgeborenen fehlt.

Schlussfolgerung. MAD hat einen Einfluss auf zerebrale Oxygenierung bei Frühgeborenen. Die Überwachung des MADs bereits während der Erstversorgung der Frühgeborenen könnte für den klinischen Alltag relevant sein, um mit der eventuellen Therapie die zerebrale Oxygenierung positiv zu beeinflussen.

\section{FV23}

\section{Peri-/intraventrikuläre Blutung bei Frühgeborenen: Einfluss der} zerebralen Oxygenierung während der Adaptationsphase

G. Pichler', B. Urlesberger', B. Schwaberger', G. Schmölzer', A. Avian' ${ }^{3}$, N. Baik ${ }^{1}$ 'Universitäts Klinik für Kinder- und Jugendheilkunde Graz, Abteilung für Neonatologie, Graz, Österreich, ${ }^{2}$ Department of Neonatology, Royal Alexandra Hospital, Edmonton, Canada, Edmonton, Kanada, ${ }^{3}$ Medizinische Universität Graz, Medizinische Informatik und Statistik, Graz, Österreich

Fragestellung. Ziel dieser Studie war es das Auftreten von peri-/intraventrikulärer (P/IVH) Blutung bei Frühgeborenen (FG) und den möglichen Zusammenhang mit zerebraler regionaler Oxygenierung ( $\left.\mathrm{CrSO}_{2}\right)$ während der Adaptationsphase zu untersuchen.

Material und Methode. In dieser prospektiven Kohortenstudie wurden Frühgeborene ( $<32$ Schwangerschaftswochen) eingeschlossen. Die zerebrale regionale Oxygenierung ( $\left.\mathrm{crSO}_{2}\right)$ wurde mittels Nahinfrarot-Spektroskopie (NIRS) mit dem INVOS $5100 \mathrm{C}$ während der neonatalen Adap- 
tationsphase (15 Minuten unmittelbar nach der Geburt) gemessen. Der Sensor wurde an der Stirn links frontal angelegt. Ferner wurde präduktal ein Pulsoxymeter angelegt, um die arterielle Sauerstoffsättigung ( $\left.\mathrm{SpO}_{2}\right)$ und die Herzfrequenz (HF) zu überwachen. Ultraschalluntersuchungen des Gehirns wurden am Tag 4, 7, 14 nach der Geburt und vor der Entlassung durchgeführt, um peri-/intraventrikuläre Blutung zu identifizieren. Je nach Ultraschalluntersuchungsergebnissen wurden die FG in zwei Gruppen eingeteilt: IVH-Gruppe und Non-IVH-Gruppe. Um die zwei Gruppen vergleichbar zu machen, wurden FG mit IVH zu den FG ohne IVH im Bezug auf Gestationsalter ( \pm 1 Woche) und Geburtsgewicht $( \pm 100 \mathrm{~g})$ gepaart.

Ergebnisse. 49 FG wurden in die Studie eingeschlossen: 12 der FG entwickelten IVH. Diese wurden mit 12 FG ohne IVH aus der Non-IVHGruppe $(n=37)$ verglichen. Es gab keinen Unterschied zwischen den zwei Gruppen in Bezug auf $\mathrm{SpO}_{2}$ und HF. Die IVH Gruppe zeigte statistisch signifikant niedrigere $\mathrm{crSO}_{2}$-Werte als die Non-IVH-Gruppe ab der Minute 6 bis zum Ende der Beobachtungsphase.

Schlussfolgerung. Die Frühgeborenen der IVH-Gruppe zeigten signifikant niedrigere $\mathrm{CrSO}_{2}$-Werte während der Adaptationsphase, obwoh es keinen Unterschied in $\mathrm{SpO}_{2}$ und HF gab. Das zusätzliche Monitoring der zerebralen regionalen Oxygenierung mittels Nahinfrarot-Spektroskopie während der Adaptationsphase könnte hilfreich sein, um die Frühgeborenen mit einem höheren Risiko für spätere IVH-Entwicklung bereits während der Adaptationsphase zu erkennen.

\section{ECMO}

\section{FV24}

Auswirkungen einer miniaturisierten Diagonalpumpe auf Hämolyse und Gerinnungsaktivierung im Tiermodell der neonatalen ECMO

S. Herber-Jonat', K. Lüninghöner', J. Ngmus', K. Förster', A. Schulze', A. Flemmer 'Dr. von Haunersches Kinderspital, Neonatologie der Kinderklinik am Perinatalzentrum Großhadern, München, Deutschland, ${ }^{2}$ Klinikum der Universität München, Klinik für Anästhesiologie, München, Deutschland

Hintergrund. Die Herausforderung der extrakorporalen Membranoxygenierung (ECMO) bei Neugeborenen mit respiratorischem Versagen ist die Notwendigkeit für miniaturisierte ECMO-Kreisläufe, Kanülen mit geringem Durchmesser und niedrige Blutflussraten. Neuartige kleine, rotierende Pumpenköpfe mit diagonalem Durchfluss und reduziertem Füllvolumen sind im Hinblick auf ihren Einsatz beim Neugeborenen vielversprechend. Bisher existieren jedoch nur wenige Daten über ihren Einfluss auf Hämolyse, Koagulation und Fibrinolyse unter standardisierten In-vivo-Bedingungen.

Methoden. Zwölf neugeborene Ferkel erhielten randomisiert entweder eine venoarterielle ECMO mit einem neuartigen diagonalen Pumpsystem oder dienten als Kontrollgruppe. Der extrakorporale Kreislauf verlief über eine $8 \mathrm{~F}-$ Kanüle in der $\mathrm{V}$. jug. ext. und eine $6 \mathrm{~F}$ in der $\mathrm{A}$. carotis. Die Durchflussrate entsprach $75 \%$ des Total Cardiac Output (8o$100 \mathrm{ml} / \mathrm{kg}$ ). Zielkriterien waren die Systemparameter des artifiziellen Kreislaufsystems und die Effekte auf Hämolyse und das Gerinnungssystem im zeitlichen Verlauf über $8 \mathrm{~h}$.

Ergebnisse. Innerhalb der ersten Stunde konnte bei allen Ferkeln der Versuchsgruppe $(n=6)$ eine Erhöhung des freien Hämoglobins beobachtet werden [49,7 mg/dl (9,0; 90,3), Mittelwert (95\% CI)]. Nach 8 h zeigte sich ein Abfall des $\mathrm{fHb}$. Allerdings war der Mittelwert weiterhin signifikant höher als bei den Kontrolltieren, welche über den Zeitraum dieselbe Menge an Fremdblut infundiert bekommen haben $[25,5 \mathrm{mg} / \mathrm{dl}$ $(8,7 ; 42,5)$ vs. $4,7 \mathrm{mg} / \mathrm{dl}(0,8 ; 8,6) ; \mathrm{p}=0,02]$. Die mediane Flussrate, der venöse Sog und die Drehzahl des Pumpenkopfs lagen bei $222 \mathrm{ml} / \mathrm{min}$ (Range: 197; 313), $-20 \mathrm{~cm} \mathrm{H} 2 \mathrm{O}$ (Range: -36 ; -6 ), und $5295 \mathrm{rpm}$ (Range: 4906; 6816). Die Spiegel für Fibrinogen und Fibrinspaltprodukte blieben sowohl bei den ECMO-Tieren als auch den Kontrollen im Normbereich.
Schlussfolgerung. Die Verwendung eines neuen diagonalen Pumpensystems für ECMO im In-vivo-Modell erzeugt einen vergleichbaren Anstieg des $\mathrm{fHb}$ wie zuvor unter In-vitro-Bedingungen beobachtet wurde.

\section{FV25}

Auswirkungen einer miniaturisierten Diagonalpumpe auf Hämolyse und Gerinnungsaktivierung bei Neugeborenen mit ECMO zur Therapie eines respiratorischen Versagens

S. Herber-Jonat', K. Förster', M. Klemme', A. Schulze', A. Flemmer'

'Dr. von Haunersches Kinderspital, Neonatologie der Kinderklinik am Perinatalzentrum Großhadern, München, Deutschland

Hintergrund. Die extrakorporale Membranoxygenierung (ECMO) bei Neugeborenen mit respiratorischem Versagen zeichnet sich durch über periphere Gefäße platzierte, kleinkalibrige Kanülen und niedrige Blutflussraten aus. In-vitro-Bench-Studien miniaturisierten Pumpsysteme mit diagonalem Blutfluss sowie der kurzfristige Einsatz dieser Systeme zum kardiopulmonalen Bypass bei pädiatrischen Patienten sind vielversprechend. Bisher existieren jedoch nur wenige Daten über die Wirkung dieser Systeme auf die Hämolyse und Gerinnungsaktivierung in der definierten Gruppe von Neugeborenen mit respiratorischem Versagen.

Studiendesign. In einer prospektiven Kohortenstudie von Neugeborenen mit venoarterieller ECMO zur Therapie des respiratorischen Versagens wurden die Systemparameter des artifiziellen Kreislaufs, das freie Hämoglobin ( $\mathrm{fHb}$ ), die Lactatdehydrogenase und die Gerinnungsaktivierung bei Verwendung der $\mathrm{DP}_{3}$ (Medos Medizintechnik AG) systematisch untersucht.

Ergebnisse. Bisher erhielten zwölf Neugeborene mit respiratorischem Versagen im Perinatalzentrum Großhadern 328o g [2700; 438o] (Median, Min-Max) eine venoarterielle ECMO mit der $\mathrm{DP}_{3}$. Verwendet wurden 8-10 Fr venöse und $8 \mathrm{~F}$ arterielle Kanülen, welche über die Halsgefäße zentral platziert wurden. Die mediane ECMO Dauer betrug 4,1 d [2;7,3 d]. Die mediane Flussgeschwindigkeit lag bei $259 \mathrm{ml} / \mathrm{min}$ [215; 314]. Der venöse Sog betrug im Median $-9 \mathrm{~cm} \mathrm{H} 2 \mathrm{O}[-50 ;-1]$ bei einer Drehzahl von $4774 \mathrm{rpm}$ [4008; 6801]. Innerhalb der ersten $24 \mathrm{~h}$ zeigten alle Patienten eine Erhöhung des fHB ( $\mathrm{fHb} 12,1 \mathrm{mg} / \mathrm{dl}$ [7,0; 70,9]) mit einem weiteren Anstieg nach 48 Stunden. Zwei Patienten entwickelten laborchemisch eine disseminierte intravasale Koagulation begleitet von einer anhaltenden Hämolyse. Zwei Patienten verstarben nach Beendigung der ECMO im Rahmen ihrer Grunderkrankung (alveolokapilläre Dysplasie, schwere Lungenhypoplasie).

Schlussfolgerung. Die Verwendung einer miniaturisierten Diagonalpumpe in Kombination mit kleinen venösen und arteriellen Kanülen im Rahmen der neonatalen ECMO erscheint sicher und wirksam. Allerdings birgt eine länger andauernde Verwendung des Systems das Risiko einer verstärkten Hämolyse und disseminierten Gerinnung.

\section{FV26}

Anwendung additiver Therapien beim schweren Lungenversagen des Neugeborenen in Deutschland

S. Demirakca', C. Patry', S. Hien', J. Reinhard', T. Schaible'

'Universitätsmedizin Mannheim, Klinik für Neonatologie, Mannheim, Deutschland

Hintergrund. Ein schweres neonatales Lungenversagen führt zum Einsatz von additiven Therapien, wie der Applikation von Surfactant, inhalativem Stickstoffmonoxid (iNO), Hochfrequenz-Oszillationsbeatmung (HFOV) oder der extrakorporalen Membranoxygenierung (ECMO). Ziel dieser Studie war es Anwendung und Effektivität dieser additiven Therapien zu dokumentieren.

Patienten und Methodik. Einschlusskriterien waren Gestationsalter $>34$ SSW, akutes Lungenversagen unter invasiver Beatmung mit $\mathrm{FiO}_{2}>0,4$ und Anwendung mindestens einer additiven Therapie. Über die Erhebungseinheit für seltene pädiatrische Erkrankungen in Deutschland (ESPED) wurden $\mathrm{n}=397$ Patienten innerhalb von 2 Jahren in die Studie 
aufgenommen. Die Effektivität der jeweiligen additiven Therapie wurde durch den behandelnden Arzt beurteilt. Vorgabe für einen positiven Effekt war eine Senkung des Oxygenierungsindex um 10 oder Anstieg der $\mathrm{PaO}_{2} / \mathrm{FiO}_{2}$ Ratio um $50 \mathrm{mmHg}$.

Ergebnisse. Die häufigste Diagnose war der Surfactantmangel mit 36,8\%, gefolgt von Pneumonie/Sepsis (16,4\%), Mekoniumsaspirationssyndrom und der kongenitalen Zwerchfellhernie. Surfactant wurde in $77,3 \%$ appliziert mit einer Effektivität von 71,6\%. 42,2\% aller Patienten wurden mit iNO behandelt, mit einer Wirksamkeit von 53,3\%. HFOV wurde bei $33,3 \%$ aller Fälle mit einer Wirksamkeit von $61,4 \%$ angewendet. Die ECMO-Therapie kam bei 14,9\% der Patienten zum Einsatz und war in $81,4 \%$ erfolgreich. Die Gesamtsterblichkeit innerhalb der Kohorte lag bei 10,3\%. Signifikante Einflussfaktoren auf das Überleben waren: die zugrundeliegende Diagnose, die Anzahl additiver Therapien und der initiale Effekt additiver Therapien ohne ECMO. 29 Patienten starben ohne ECMO-Therapie. Davon lag in 10 Fällen eine ECMO Kontraindikation vor. Die restlichen 19 Patienten, die womöglich von einer ECMO-Therapie profitiert hätten wurden nicht in ein ECMO-Zentrum überwiesen.

Schlussfolgerung. Die Anwendung und Effektivität additiver Therapien beim Lungenversagen des Neugeborenen entspricht in Deutschland den international vorliegenden Daten. Jedoch zeigt sich, dass jährlich eine bedeutende Anzahl Neugeborener mit ECMO-Indikation nicht an ein entsprechendes Zentrum verlegt wird.

\section{FV27 \\ Diagnoseprofil und aktuelle Ergebnisse der neonatalen extra- korporalen Membranoxygenierung}

S. Hien', J. Reinhard', S. Demirakca', V. Varnholt', T. Schaible'

'Klinikum Mannheim, Klinik für Neonatologie, Mannheim, Deutschland

Hintergrund. Beim Lungenversagen des Neugeborenen haben schonende Beatmung und die additiven Therapien Surfactant und Stickstoffmonoxid den Bedarf für die extrakorporale Membranoxygenierung (ECMO) reduziert. Die erworbenen Atemstörungen Mekoniumaspiration und Pneumonie/Sepsis sind dadurch beherrschbarer geworden, womit die angeborenen Atemstörungen mehr ins Visier für die ECMO treten. Die folgende Analyse soll die Frage beantworten wie das Diagnoseprofil für ein ECMO Zentrum heutzutage aussieht und ob sich dadurch die Überlebenszahlen geändert haben.

Patienten und Methodik. In den Jahren 2005-2014 wurden an unserem Zentrum 294 Neugeborene mit der ECMO behandelt. Diese Patienten wurden hinsichtlich der ursächlichen Diagnose und des Überlebens ausgewertet. Für die angeborene Zwerchfellhernie wurde konsequent ein standardisierter Therapiealgorithmus angewendet.

Ergebnisse. 219 von 294 ECMO Patienten (74,5\%) haben überlebt und konnten nach Hause entlassen werden. Damit hat sich die Gesamtüberlebensrate im Vergleich zu früheren Daten und den Daten der Extracorporeal Life Support Organisation (ELSO) leicht verbessert. Und das obwohl die angeborene Zwerchfellhernie (CDH) mit 200 Fällen noch deutlicher zur führenden Diagnose geworden ist. Die Überlebensrate der $\mathrm{CDH}$ liegt mit 75,5\% sogar minimal über dem Durchschnitt und deutlich über den Ergebnissen der ELSO. Es zeigt sich die Besonderheit unseres Zentrums mit der Spezialisierung auf die Diagnose CDH mit durchschnittlich 2o ECMO Fällen bei dieser Diagnose pro Jahr, wobei ca. ein Drittel der prä-und postnatal verlegten CDH-Kinder mit ECMO behandelt werden. Aus allen anderen Diagnosegruppen ergaben sich zusammen durchschnittlich 1o ECMO Fälle pro Jahr. Hierbei erfüllten zwei Drittel der seit 2005 abgeholten Neugeborenen die ECMO-Kriterien und ein Drittel konnte mittels Optimierung der Therapie stabilisiert werden konnten. Die Mekoniumaspiration (MAS) ist mit 16,3\% der ECMO Fälle die zweithäufigste Diagnose nach der $\mathrm{CDH}$ und hierbei überlebten $96 \%$ der Patienten. Deutlich schlechtere Überlebensraten zeigten die Diagnosen primäre persistierende pulmonale Hypertension (PPHN) $[8,2 \%$ der Patienten] mit nur $38 \%$ und die Pneumonie/ Sepsis $[6,2 \%]$ mit $55,5 \%$. Von 4 Kindern mit sonstigen Diagnosen überlebten 3. Gerade bei der PPHN fällt der mit durchschnittlich 3 Lebenstagen relativ späte Verlegungszeitpunkt auf.
Schlussfolgerung. Ein optimierter Therapiealgorithmus und die hohe Spezialisierung mit hohen Fallzahlen haben bei der Diagnose $\mathrm{CDH}$ zu deutlich verbesserten ECMO- Überlebensraten geführt. Bei allen anderen Diagnosen sind die Fallzahlen für ECMO rückläufig und es zeigt sich, dass die Therapieabläufe für die PPHN verbessert werden sollten.

\section{Neonatologie: NIDCAP}

\section{FV28}

Fünf Jahre psychologisch-sozialmedizinische Versorgung an einem Perinatalzentrum - II. Auswirkungen auf die stationären Behandlungskosten von frühgeborenen Kindern

J. Reichert', M. Schemken ${ }^{2}$, R. Manthei' ${ }^{2}$, J. Schmitt ${ }^{3}$, M. Rüdiger ${ }^{1}$

${ }^{1}$ Medizinische Fakultät Carl Gustav Carus an der TU Dresden, Klinik und Poliklinik für Kinder- und Jugendmedizin, Neonatologie und Pädiatrische Intensivmedizin, Dresden, Deutschland, ${ }^{2} \mathrm{AOK}$ Plus Sachsen und Thüringen, Dresden, Deutschland, ${ }^{3}$ Medizinische Fakultät Carl Gustav Carus an der TU Dresden, Zentrum für Evidenzbasierte Gesundheitsversorgung, Dresden, Deutschland

Hintergrund. Eine adäquate psychologisch-sozialmedizinische Versorgung von Familien Frühgeborener bereits während des initialen stationären Aufenthalts soll dazu beitragen, geburtsbedingte Anpassungs- und Belastungsstörungen der Eltern zu vermeiden, den Eltern-Kind-Bindungsprozess zu unterstützen sowie die medizinischen Behandlungserfolge langfristig zu sichern (Reichert \& Rüdiger. Psychologisch-sozialmedizinische Versorgung von Eltern Frühgeborener. Stuttgart: Ligatur 2012). Mit diesen Zielstellungen werden auch Auswirkungen auf stationäre Behandlungskosten insbesondere in Bezug auf stationäre Wiederaufnahmen im 2. Lebensjahr erwartet.

Zielstellung. Analyse stationärer Behandlungskosten der ersten beiden Lebensjahre nach Einführung einer strukturierten Psychologisch-sozialmedizinischen Versorgung von Familien Frühgeborener (PSV) am Perinatalzentrum Dresden im Jahr 2009 im Vergleich zu anderen Zentren ohne PSV.

Material und Methode. Erhoben wurden die stationären Behandlungskosten für die ersten beiden Lebensjahre aller Kinder der Geburtsjahrgänge 2008 und 2010, die im Freistaat Sachsen geboren wurden $(\mathrm{N}=34.411$ bzw. $\mathrm{N}=35.091)$ und bei der AOK Plus versichert waren $(\mathrm{N}=14.723,42,8 \% ; \mathrm{N}=14.984,42,7 \%)$. Kinder mit unterbrochenem Versicherungsverlauf wurden von der Analyse ausgeschlossen.

Ergebnisse. Von den 13.259 eingeschlossenen Kindern des Geburtsjahrganges 2008 wurden 573 (darunter 35 VLBW- und 88 LBW-Kinder) im Perinatalzentrum Dresden und 12.575 (103 bzw. 658) in anderen Zentren versorgt. Für 2010 mit 13.705 eingeschlossenen Kindern ergaben sich für Dresden 687 (44 bzw. 83) und für die anderen Zentren 13.018 Fälle (124 bzw. 723). Während in den anderen Zentren die stationären Behandlungskosten für VLBW-Kinder in den ersten beiden Lebensjahren im Vergleich der Geburtskohorten 2008 und 2010 um insgesamt $15.138 €$ je Kind anstiegen $(t=2,670, p=0,01)$, sanken sie am Perinatalzentrum Dresden um $7924 €$, resultierend vor allem aus Einsparungen im 2. Lebensjahr (ca. $2000 € \mathrm{t}=2,263, \mathrm{p}=0,03$ ). Für die LBW-Kinder anderer Einrichtungen ergaben sich von 2008 zu 2010 keine Kostendifferenzen; für die am Perinatalzentrum Dresden versorgten LBW-Kinder wurden $1770 €$ Kosteneinsparungen ermittelt.

Diskussion. Nach der PSV-Einführung am Perinatalzentrum Dresden im Jahre 2009 wurden für die hier versorgten VLBW- und LBW-Kinder deutliche bzw. messbare Kosteneinsparungen für stationäre Behandlungen innerhalb der ersten beiden Lebensjahre festgestellt, die sich von Kostenentwicklungen anderer Einrichtungen unterscheiden. Die Daten können eine verbesserte und längerfristig gesicherte gesundheitliche Entwicklung der Kinder anzeigen, zu der eine adäquate PSV ihrer Familien beigetragen haben kann. 
Schlussfolgerung. Die Ergebnisse stützen die Annahme positiver und an den Kosten messbarer Wirkungen einer PSV von Familien Frühgeborener. Allerdings wird deren Beitrag vor dem Hintergrund der Veränderungen in der medizinischen Behandlung Frühgeborener noch zu prüfen sein.

\section{Pädiatrische Intensivmedizin: Folgen von Sedierung und Narkose}

\section{FV29 \\ Risikofaktoren für die Entstehung eines Delirs bei pädiatrischen Intensivpatienten}

\section{J. Meyburg', M. Dill', S. Picardi', J. Westhoff', W. Springer ${ }^{3}$, C. Traube ${ }^{4}$, R. von Haken $^{5}$}

'Zentrum für Kinder- und Jugendmedizin, Allgemeine Pädiatrie, Heidelberg, Deutschland, ${ }^{2}$ Universitätsklinikum Heidelberg, Klinik für Anästhesiologie, Heidelberg, Deutschland, ${ }^{3}$ Universitätsklinik Heidelberg, Klinik für Kinder-Jugendmedizin, Heidelberg, Deutschland, ${ }^{4}$ Weill Cornell Medical College, Pediatric Critical Care, New York, USA, ${ }^{5}$ Universitätsklinikum Heidelberg, Klinik für Anästhesiologie, Heidelberg, Deutschland

Hintergrund. Als Delir wird ein komplexes neurologisches Krankheitsbild mit Bewusstseins-, Wahrnehmungs- und/oder kognitiven Störungen bezeichnet, das beim erwachsenen Intensivpatienten eine große Bedeutung besitzt. Inzidenz und Risikofaktoren für die Entstehung bei Kindern sind noch weitgehend unbekannt.

Fragestellung. Welche Faktoren erhöhen das Risiko für ein Delir bei pädiatrischen Intensivpatienten?

Material und Methoden. Im Rahmen einer prospektiven Studie wurden 93 Patienten [3,5 $\pm 4,7$ Jahre (o-17), m:w=47:46] untersucht, die nach einer geplanten Operation auf die pädiatrische Intensivstation aufgenommen wurden. Sobald die Patienten ansprechbar waren (RASS $>-3$ ), wurde über 5 Tage 2-mal täglich ein Delirscore (deutsche Version des Cornell Assessment of Pediatric Delirium) erhoben. Aufgrund der Ergebnisse wurden die Patienten in drei Gruppen eingeteilt (kein, leichtes oder schweres Delir).

Ergebnisse. Insgesamt wiesen 61 Patienten (66\%) ein Delir auf, davon 30 (32\%) ein leichtes und 31 (33\%) ein schweres. Das Geschlechterverhältnis war in allen Gruppen ausgeglichen. Jüngere Kinder entwickelten häufiger ein Delir und die Symptome waren ausgeprägter (Säuglinge: leichtes Delir 43\%/schweres Delir 43\%; Kleinkinder: 20\%/37\%; Schulkinder: $33 \% / 11 \%$; Jugendliche: $33 \% /-)$. Im Gegensatz zu Erwachsenen stellten Anzahl vorausgegangener Operationen oder eine positive Deliranamnese keine Risikofaktoren dar. Eine total intravenöse Anästhesie (TIVA) ging mit einem niedrigeren Risiko für ein Delir einher als eine balancierte Anästhesie $(\mathrm{p}<0,05)$, Hypothermie, Blutverlust oder extrakorporaler Kreislauf hatten diesbezüglich keine Auswirkungen. Bei vergleichbarer Narkosedauer wurden Patienten mit Delir signifikant länger nachbeatmet $(\mathrm{p}<\mathrm{0}, \mathrm{O01})$. Deutliche Unterschiede fanden sich zwischen Patienten mit leichtem und schweren Delir sowie im Vergleich zur Kontrollgruppe in der kumulativen Dosis zahlreicher Medikamente. Beispielsweise gingen hohe Dosen von Sevofluran und länger wirksame Sedativa wie Midazolam und Fentanyl mit einer erhöhten Delirinzidenz einher, bei kürzer wirksamen Substanzen wie Propofol oder Sufentanil war dies nicht zu beobachten $(\mathrm{p}<\mathrm{0}, \mathrm{o01})$. Die Anzahl der Fremdkörper korrelierte signifikant mit den Delirscores, wobei bestimmte Fremdkörper (CPAP, Magensonde, ZVK, Arterie, Blasenkatheter) schlechter toleriert wurden als andere (Drainagen, Cystofix, EVD, PVK, $\mathrm{p}<\mathrm{o}, \mathrm{o1}$ ). Infektionen erhöhten das Delirrisiko signifikant $(\mathrm{p}<\mathrm{0}, 001)$.

Schlussfolgerung. Die systematische Untersuchung lässt auch bei pädiatrischen Intensivpatienten eine hohe Delirinzidenz erkennen. Kinder mit einem Delir hatten einen erhöhten Pflegeaufwand sowie signifikant verlängerte Aufenthalte auf der Intensivstation sowie im Krankenhaus insgesamt. Nachbeatmung, Medikamente, Fremdkörper und Infektio- nen sind die wichtigsten Risikofaktoren für die Ausbildung eines Delirs. Unsere Daten können als Grundlage für zukünftige Präventionsund Behandlungsstrategien dienen.

\section{Kreislauftherapie}

\section{FV30 \\ Evaluation und Weiterentwicklung eines klinischen Scores zur Diagnose des hämodynamisch bedeutsamen persistierenden Ductus arteriosus (PDA) bei Frühgeborenen $<\mathbf{3 4 + 0}$ SSW}

A. Kindler', B. Seipolt'², A. Heilmann', J. Dinger'², M. Rüdiger', S. Hofmann²

'Universitätsklinikum Carl Gustav Carus Kinderklinik, Dresden, Deutschland, ${ }^{2}$ Univ. Klinikum Carl Gustav Carus, Klinik und Poliklinik für Kinder- und Jugendmedizin, Neonatologie, Pädiatrische Intensivmedizin, Dresden, Deutschland

Hintergrund. Der Ductus arteriosus Botalli stellt in den ersten 2-3 Lebenstagen bei gesunden Neugeborenen eine noch physiologische Shuntverbindung dar. Der postpartale Anstieg des Sauerstoffpartialdruckes und ein Absinken von Prostaglandin E1 (PGE1) führt zur Konstriktion der duktalen Gefäßmuskulatur. Bei Frühgeborenen (FG) ist die Sensitivität für Sauerstoff verringert und für PGE1 bzw. Stickstoffmonoxid (NO) erhöht. Die physiologische Abnahme des pulmonalen Widerstandes führt innerhalb der ersten Lebensstunden zu einer vermehrten Lungendurchblutung und zu vermehrtem Links-Rechts-Shunt (LRS) über den PDA. Dieser kann folgende hämodynamische Auswirkungen haben: pulmonale Überflutung, Minderperfusion der vom Systemkreislauf versorgten Organe sowie eine kardiale Belastung.

Fragestellung. Evaluierung eines klinischen PDA-Scores, der von Kabus M 1991 beschrieben und in unserer Klinik in den letzten Jahren weiterentwickelt wurde, im Hinblick auf seine Vergleichbarkeit zur Aussagefähigkeit der Echokardiographie hinsichtlich der hämodynamischen Relevanz des PDA.

Methodik. Das Patientenkollektiv umfasst 154 Frühgeborene mit einem Gestationsalter von $<34+0$ SSW. Der Erfassungszeitraum der Daten erstreckt sich von Oktober 2008 bis September 2010. Die Auswertung basiert auf einem im Vergleich zu Kabus M (1991) vereinfachten PDA-Score. Täglich wird ab dem zweiten Lebenstag bis zum zehnten Lebenstag der PDA-Score erhoben, wobei definierte klinische Parameter beurteilt werden: Herzgeräusch, sichtbare präkordiale Pulsationen, Herzfrequenz $>160 / \mathrm{min}$ (ohne Hypovolämie), Apnoen oder Respiratortherapie, kräftige periphere Pulse, Hepatomegalie, metabolische Azidose, respiratorische Verschlechterung. Es wird pro positivem Merkmal ein Punkt im Score-System vergeben. Bei Erreichen eines Ductus-Scores von $\geq 2$ Punkten wird eine echokardiographische Untersuchung (als Goldstandard in der Diagnostik des PDA) initiiert.

Ergebnisse. Das mittlere Gestationsalter der Studiengruppe beträgt 28,11 SSW ( $\sigma 2,23$ SSW). Das mittlere Geburtsgewicht liegt bei 1052,05 g $(\sigma 373,70 \mathrm{~g})$. Die Gruppe setzt sich zusammen aus 59,7\% männlichen sowie 40,3\% weiblichen Neonaten. Um die einzelnen Parameter des vereinfachten PDA-Scores zu wichten, wurden mittels Kreuztabellen die klinischen Bestandteile des Scores jeweils einzeln der Entwicklung eines echokardiogaphisch bestätigten hrPDA gegenübergestellt. Durch die Ermittlung des angepassten Residuums, sowie der exakten Signifikanz mittels Fisher-Test konnten die signifikanten, statistisch stärksten Einzelparameter ermittelt werden. Ein Versuch der Zusammenfassung der klinischen Merkmale mittels logistischer Regressionsanalyse konnte einzelne Kernmerkmale, wie metabolische Azidose, präkordiale Pulsationen, periphere Pulse sowie Apnoe bzw. Respiratortherapie (pulmonale Verschlechterung) herauskristallisieren. Am dritten und vierten Lebenstag korrelieren diese Parameter am besten mit der Entwicklung eines echokardiographisch bestätigten hrPDA.

Schlussfolgerung. Der von uns getestete klinische PDA-Score ist in der Anwendung einfach, nichtinvasiv und schnell durchführbar. In 
Kombination mit der kontinuierlichen Erfassung dieses klinischen PDA-Scores lässt sich die Echokardiographie gezielter einsetzen und gegebenenfalls die Anzahl der notwendigen echokardiografischen Untersuchungen reduzieren.

\section{FV31}

\section{Selektive $\beta 1$-Blockade bei Akzeptoren eines schweren,} chronischen fetofetalen Transfusionssyndromes (FFTS)

\section{K. Gründler', W. Baden', K. Kagan'3 , C. Poets', A. Franz'}

'Eberhard-Karls-Universität, Universitätsklinik für Kinderheilkunde und Jugendmedizin, Neonatologie, Tübingen, Deutschland, ${ }^{2}$ Eberhard-Karls-Universität, Universitätsklinik für Kinderheilkunde und Jugendmedizin, Kinderkardiologie, Tübingen, Deutschland, ${ }^{3}$ Eberhard-Karls-Universität, Universitätsklinik für Frauenheilkunde, Perinataldiagnostik, Tübingen, Deutschland

Hintergrund. In 10-15\% aller monochorialen Zwillingsschwangerschaften kommt es über plazentare Anastomosen zur Entwicklung eines unbalancierten, chronischen Bluttransfers zwischen den Zwillingen. Dies ist mit hoher perinataler Mortalität und Morbidität assoziiert. Um Überleben und Langzeitprognose zu verbessern, scheint auch die Therapie der kardialen Funktionsstörung des Akzeptors wichtig zu sein, in deren Mittelpunkt eine Myokardhypertrophie aufgrund erhöhter Vorlast durch chronische Hypervolämie und erhöhter Nachlast durch gesteigerten arteriellen Blutdruck steht.

Methodik. Wir beschreiben zwei aus unserer Sicht typische postnatale Verläufe von FFTS-Akzeptoren mit Myokardhypertrophie, diastolischer Funktionsstörung und Entwicklung eines Low-cardiac-outputSyndromes.

Ergebnisse. Bei unseren Patienten handelt es sich um eutrophe Zwillingsfrühgeborene aus 26+o bzw. 25+2 Schwangerschaftswochen (SSW). Im ersten Fall überlebte der hypotrophe Donor, im zweiten Fall starb der Donor sub partu. Echokardiografisch zeigte sich am 4. respektive 10. Lebenstag (LT) das Vollbild der kardialen Funktionsstörung mit biventrikulärer Myokardhypertrophie, systolisch kollabierenden Ventrikeln und linksintraventrikulärer (LV) Stenose.

Fall 1. Neben Volumengaben vom 1.-3. LT mit im Mittel $30 \mathrm{ml} / \mathrm{kg} / \mathrm{d}$ erfolgte vom 2.-8. LT die Kreislaufunterstützung mit Noradrenalin. Nach Beginn der $\beta 1$-selektiven Therapie mit Esmolol in aufsteigender Dosierung ab dem 4. LT waren am 6. LT bei maximaler Dosis $(50 \mu \mathrm{g} / \mathrm{kg} / \mathrm{min})$ zusätzliche Volumengaben bis maximal $63 \mathrm{ml} / \mathrm{kg} / \mathrm{d}$ bei schlechter Herzfüllung notwendig. Ab dem 7. LT keine zusätzliche Flüssigkeitssubstitution mehr aus kardialem Anlass. Wechsel auf Metoprolol Tartrat ab dem 17. LT, ab dem 42. LT schrittweise Dosisreduktion bei nicht mehr bestehender LV-Ausflussbahnobstruktion bis zum 63. LT.

Fall 2. Vom 1.-4. LT zusätzliche Volumengaben von im Mittel $71 \mathrm{ml} /$ $\mathrm{kg} / \mathrm{d}$ und vom 6.-12. LT von im Mittel $74 \mathrm{ml} / \mathrm{kg} / \mathrm{d}$ notwendig, zudem vom 1.-4. LT, 6.-15. LT und 17.-20. LT Kreislaufunterstützung mit Noradrenalin. Nach Beginn von Esmolol ab dem 11. LT keine zusätzliche Volumengabe mehr ab dem 13. LT. Wechsel auf Metoprolol Tartrat ab dem 27. LT, ab dem 58. LT schrittweise Dosisreduktion bei regredienter LV-Hyperkontraktilität und Hypertrophie bis zum 69. LT.

Schlussfolgerung. Die Behandlung der kardialen Funktionsstörung der Akzeptoren des FFTS mit hypertroph-obstruktiver Kardiomyopathie mit selektiven $\beta_{1}$-Rezeptorantagonisten und intermittierend Noradrenalin zur Steigerung des Blutdruckes erschien, gemessen an der nachfolgenden Regredienz des Flüssigkeitsbedarfes und der echokardiografischen Befunde, erfolgreich. Neben der seriellen Überwachung des linksventrikulären Auswurfs anhand des "velocity time intergral" (VTI) über der Aortenklappe sollten zukünftig auch die echokardiografischen Parameter der diastolischen Funktion seriell überwacht werden.
FV32

Effekt von Thrombozytentransfusionen auf den Verschluss eines offenen Duktus arteriosus bei Frühgeborenen

V. Wiebking', A. Florian' ${ }^{2}$, M. Sperandio 3 , A. Flemmer ${ }^{2}$

${ }^{1}$ Klinikum der Universität München, Dr. von Haunersches Kinderspital, München, Deutschland, ${ }^{2}$ Klinikum der Universität München, Neonatologie der Kinderklinik am Perinatalzentrum Großhadern, München, Deutschland, ${ }^{3}$ Ludwig-Maximilians-Universität München, Walter-Brendel-Zentrum für Experimentelle Medizin, München, Deutschland

Hintergrund. Der Verschluss des Ductus arteriosus bei reifen Neugeborenen ist mit der Bildung eines stabilen Thrombus assoziiert. Bei Frühgeborenen unter $1500 \mathrm{~g}$ Geburtsgewicht verschließt sich ein offener Duktus arteriosus (PDA) häufig nicht spontan, so dass bei hämodynamischer Relevanz ein medikamentöser oder operativer Verschluss notwendig werden kann. Es ist bisher unklar, ob die postnatale Transfusion von adulten Thrombozyten den spontanen oder medikamentös induzierten Verschluss des PDA beeinflusst.

Fragestellung. In einer retrospektiven Datenbankanalyse wurde untersucht, ob die Transfusion adulter Spender-Thrombozyten innerhalb der ersten Lebenstage den Verschluss eines hämodynamisch relevanten persistierenden Duktus arteriosus (HRPDA) bei Frühgeborenen mit extrem geringem Geburtsgewicht und bereits begonnener Indomethacin-Therapie begünstigt.

Material und Methoden. Frühgeborene mit einem Geburtsgewicht unter $1500 \mathrm{~g}$ und einem Gestationsalter von bis zu 32 Wochen, die zwischen 1998 und 2013 geboren wurden, wurden retrospektiv untersucht ( $n=1209 ;$ Ausgangspopulation). Für 866/1209 (72\%) lagen alle erforderlichen Daten für eine Analyse vor. Wir definierten eine Thrombozytenzahl unter 150 G/l als Thrombozytopenie. Die Dateneingabe sowie die statistische Auswertung wurden mittels Excel 2010 (Microsoft Corp., USA) und SPSS 22 (IBM Inc., USA) durchgeführt.

Ergebnisse. Von den 866 Kindern entwickelten 291 (33,6\%) einen HRPDA und erhielten eine Therapie mit Indomethacin. Zehn dieser Kinder verstarben postnatal $(3,4 \%)$. Es bestand kein Zusammenhang zwischen der Schwere der Thrombozytopenie und dem Auftreten eines HRPDA. Der erfolgreiche medikamentöse Verschluss eines HRPDA war unabhängig von einer postnatal beobachteten Thrombozytopenie $(27.8 \%$ vs. $34.8 \%$, mit/ohne Thrombozytopenie, $\mathrm{p}=0,325)$. Die Transfusion von adulten Thrombozyten hatte keinen Einfluss auf die Notwendigkeit einer Duktusligatur (36,4\% mit vs. $32,4 \%$ ohne Transfusionen, $p=0,699)$. Eine Analyse nur der überlebenden Kinder erbrachte ein ähnliches Ergebnis (42,1\% mit vs. $32,8 \%$ ohne Transfusionen; $p=0,408)$. Wesentlicher prädiktiver Faktor für das Versagen einer medikamentösen Duktus-Therapie war das postmenstruelle Alter bei Geburt $(\mathrm{p}<\mathrm{0}, \mathrm{O0})$.

Diskussion. Unsere Daten zeigen, dass eine postnatale Transfusion von adulten Thrombozyten keinen Effekt auf den Erfolg oder Misserfolg einer Indomethacin-Therapie bei HRPDA hat. Unsere Daten widerlegen außerdem frühere Beobachtungen, die einen Zusammenhang einer Thrombozytopenie mit dem Auftreten eines HRPDA implizierten. Eine Limitation dieser retrospektiven Analyse ist, dass ein signifikanter Unterschied zwischen thrombozytopenen und nicht-thrombozytopenen Kindern hinsichtlich wesentlicher klinischer Parameter (Gestationsalter, Geburtsgewicht, Hirnblutungsrate und Mortalität) bestand, so dass anhand der vorgelegten Daten keine abschließenden Schlüsse gezogen werden können. 


\section{FV33}

Photoplethysmography Imaging (PPGI) - eine kontaktlose Messmethode zur funktionellen Erfassung der lokalen Hämodynamik des Frühgeborenen

\begin{abstract}
K. Heimann', N. Blanik², C. Pereira², M. Wester', V. Blazek², T. Orlikowsky ${ }^{3}$
'Universitätsklinikum Aachen, Sektion Neonatologie, Klinik für Kinder- und Jugendmedizin, Aachen, Deutschland, ${ }^{2} \mathrm{Helmholtz}$ Institut der RWTH Aachen, Lehrstuhl für Medizinische Informationstechnik, Aachen, Deutschland, ${ }^{3}$ Universitätsklinik für Kinder- und Jugendmedizin, Sektion für Neonatologie, Aachen, Deutschland
\end{abstract}

Einleitung. Die Registrierung der lokalen hämodynamischen Verhältnisse bei Frühgeborenen mit konsekutiver Änderung der Oxygenierung ist ein Bestandteil der neonatologischen Forschung, um Zustandsveränderungen (Sepsis, Zentralisierung bei NEC etc.) möglichst früh zu erfassen. Die bisher zur Verfügung stehenden Messmethoden sind entweder invasiv- (pVK A. rad.) oder nichtinvasiv (PI, NIRS), benötigen aber alle eine direkte Verbindung zum Patienten. Mittels PPGI wird kontaktlos funktionell die Durchblutung der Hautschichten erfasst. Dieses Verfahren misst ortsaufgelöst in Abhängigkeit vom lokalen Blutvolumen mittels einer hochempfindlichen CCD Kamera inkl. Beleuchtungseinheit die optische Dämpfung der Haut. Dieses Messprinzip wurde bisher nur bei erwachsenen Patienten angewendet.

Ziel. Ist die kontaktlose ortsaufgelöste Erfassung der lokalen Durchblutung und Analyse der Methode an mehreren peripheren und zentralen Körperarealen im Inkubator in Bauch- und Rückenlage bei Frühgeborenen möglich?

Patienten und Methoden. Bei 10 spontanatmenden Frühgeborenen [Gestationsalter median bei Geburt 26 SSW (24-28); Biolog. Alter $35 \mathrm{~d}$ (8-41), Gewicht 960 g (670-1290)], 5 männlich, Messung der Durchblutung mittels PPGI (Amplitude) an 3 Körperarealen. Arm (A), Kopf (K) und Thorax (T). 3 Messpunkte, alle 10 Sekunden registriert, Korrelation mit Herzfrequenz (EKG, Pulsoxymetrie). Versuchsanordnung mit 2 Phasen à 5 Minuten: Bauchlage mit Wechsel nach standardisierter Umlagerung Rückenlage. Vor jeder Aufzeichnung Washout-Zeit von max. 1o Minuten (Minimierung Carryover-Effekte). Statistische Auswertung mittels Korrelationskoeffizient nach Lin.

Ergebnisse. Sowohl in Bauch- als auch in Rückenlage zeigt sich in allen 3 Körperarealen jeweils eine signifikante Korrelation zwischen dem Referenzsignal und der PPGI (jeweils $\mathrm{p}<0,05)$. Dabei lag die höchste Korrelation im Bereich des Kopfes (93 bzw. 97\%), gefolgt vom Thorax (83 bzw. 93\%) und der Peripherie (Arm: 67 bzw. 83\%).

Schlussfolgerung. Die PPGI eignet sich zur ortsaufgelösten kontaktlosen Erfassung der Durchblutung in unterschiedlichen Körperregionen bei Frühgeborenen. Die Ergebnisse können eine Basis zur diagnostischen Erweiterung der Beurteilung der Hämodynamik Frühgeborener sein.

\section{FV34}

Photoplethysmography Imaging (PPGI) und Infrarotthermographie (IRT) als kontaktlose Messmethoden zur kombinierten Erfassung der lokalen Hämodynamik in Abhängigkeit von der Temperatur des Frühgeborenen

\section{K. Heimann', N. Blanik', C. Pereira'2, M. Wester', V. Blazek², S. Leonhardt', T. Orlikowsky ${ }^{4}$}

'Universitätsklinikum Aachen, Klinik für Neugeborenen- und Konservative Kinderintensivmedizin, Aachen, Deutschland, ${ }^{2}$ Helmholtz Institut der RWTH Aachen, Lehrstuhl für Medizinische Informationstechnik, Aachen, Deutschland, ${ }^{3}$ Universitätsklinikum Aachen, Sektion Neonatologie, Klinik für Kinderund Jugendmedizin, Aachen, Deutschland, ${ }^{4}$ Universitätsklinik für Kinder- und Jugendmedizin, Sektion für Neonatologie, Aachen, Deutschland

Einleitung. Die PPGI eignet sich zur ortsaufgelösten kontaktlosen Erfassung der Durchblutung in unterschiedlichen Körperregionen bei Frühgeborenen (Abstract: 307). Mittels Infrarotthermographie (IRT) kann man Oberflächentemperaturen und deren Verteilungsmuster in
Abhängigkeit von der Lagerungsform messen. Die aktive Thermoregulation über Vasodilatation und -konstriktion ist beim Erwachsenen zehnmal so effektiv wie die passive Diffusion von Wärme über die einzelnen Hautschichten.

Ziel. Ist eine kontaktlose ortsaufgelöste Erfassung der lokalen Durchblutung in Abhängigkeit von der Temperaturverteilung an mehreren peripheren und zentralen Körperarealen im Inkubator in Bauch- und Rückenlage bei Frühgeborenen möglich?

Patienten und Methoden. Bei 7 spontanatmenden Frühgeborenen [Gestationsalter median bei Geburt 25 SSW (24-26); Biolog. Alter $38 \mathrm{~d}$ (29-41), Gewicht 950 g (670-1198)], 3 männlich, Messung der Durchblutung mittels PPGI (Amplitude) und der Temperaturverteilung (IRT) in 3 Körperarealen: Arm (A), Kopf (K) und Thorax (T). 3 Messpunkte, alle 10 Sekunden registriert, Korrelation mit Herzfrequenz (EKG). Versuchsanordnung mit 2 Phasen à 5 Minuten: Bauchlage (BL) mit Wechsel nach standardisierter Umlagerung zu Rückenlage (RL). Vor jeder Aufzeichnung Washout-Zeit von max. 10 Minuten (Minimierung Carryover-Effekte). Statistische Auswertung mittels Korrelationskoeffizient nach Lin.

Ergebnisse. IRT: Unabhängig von der Lagerungsform war die zentralen Körperregion jeweils um $1^{\circ} \mathrm{C}$ wärmer als die Peripherie $(\mathrm{p}<0,05)$. Dagegen war die Temperatur in ein und demselben Areal in Bauch- und Rückenlage nahezu identisch. PPGI: In sämtlichen Regionen war die Amplitude nahezu konstant mit einem Wert $<1,0$ ( $p>0,05)$. Dies jeweils unabhängig von der Lagerungsform. Es gab keinerlei Korrelation zwischen IRT und PPGI in den einzelnen Körperarealen in Bauch- oder Rückenlage ( $p>0,05)$.

Schlussfolgerung. Trotz unterschiedlicher Temperaturen in den einzelnen Körperregionen blieb die lokale Durchblutung unabhängig vom gemessenen Areal nahezu konstant. Dies könnte als Hinweis dafür gewertet werden, dass beim Frühgeborenen die Hauttemperatur weniger durch Perfusion als durch andere Mechanismen wie z. B. eine passive Wärmediffusion bestimmt wird.

\section{FV35}

Messung des echokardiographischen Flussprofils im rechtventrikulären Ausflusstrakt zur Risikoeinschätzung bei Kindern mit angeborener Zwerchfellhernie.

\section{F. Kipfmueller', L. Schröder', K. Heindel', P. Bartmann', A. Müller'}

'Zentrum für Kinderheilkunde, Universität Bonn, Neonatologie und Pädiatrische Intensivmedizin, Bonn, Deutschland

Einleitung. Ein wesentlicher prognoseentscheidender Faktor bei Kindern mit angeborener Zwerchfellhernie (CDH) ist das Ausmaß der pulmonalen Hypertonie (PH). Die transthorakale Echokardiographie ist die am häufigsten genutzte Methode zur Schweregradeinteilung einer $\mathrm{PH}$. In der Regel erfolgt dies mittels der Geschwindigkeit einer Trikuspidalinsuffizienz (TI), der Flussrichtung im Ductus arteriosus (DA) oder der Ventrikelmorphologie. Allerdings sind Quantifizierungen über TI und DA nicht bei allen Patienten möglich.

Fragestellung. Untersucht wurde der prognostische Stellenwert des Verhältnisses der Doppler-echokardiographisch gemessenen Accelerationszeit (TPV) zur gesamten rechtsventrikulären Ejektionsdauer (RVET) im rechtsventrikulären Ausflusstrakt (RVOT) in Bezug zum Vorliegen einer schweren $\mathrm{PH}$ und der Notwendigkeit einer ECMOTherapie.

Methodik. Prospektive Beobachtungsstudie bei Kindern mit CDH die zwischen Februar 2013 und Dezember 2014 in unserer Abteilung behandelt wurden. Im Rahmen der ersten Echokardiographie wurden TI, DA-Fluss und TPV/RVET gemessen. Das behandelnde Team war bzgl. der TPV/RVET Messungen verblindet. Zur Beurteilung einer PH wurden folgende Faktoren verwendet: TI, DA-Fluss, Ventrikelmorphologie und persistierende prä- und postduktale Sättigungsdifferenzen. Es erfolgte die Einteilung in folgende Gruppen: (1) ECMO; (2) Schwere PH ohne ECMO; (3) Kontrollgruppe (Keine bzw. max. moderate PH). 
Ergebnisse. Während des Beobachtungszeitraums wurden 41 Kinder mit $\mathrm{CDH}$ in unserer Abteilung behandelt. Davon konnten 35 Patienten mit einer mittleren observed-to-expected lung-to head ratio von $38,5 \%(16-60 \%)$ in die Analyse eingeschlossen werden. Zuordnung der Patienten: Gruppe 1: 13 Kinder; Gruppe 2: 7 Kinder; Gruppe 3: 15 Kinder. TPV und RVET-Ratio waren bei $100 \%$ der Kinder messbar, eine valide TI bei $62,9 \%$ und ein DA bei $94,3 \%$. Bei Patienten mit PH war eine TI in 70\% vorhanden In der ECMO-Gruppe bei 61,5\%. Die mittlere TPV/RVET lag in Gruppe 1 (ECMO) bei 0,228 , in Gruppe 2 bei 0,241 und in Gruppe 3 bei 0,323 . Als optimaler Cut-off-Wert für eine ECMO-Therapie ergab sich 0,264, für das Vorliegen einer schweren $\mathrm{PH}$ o,294. Sensitivität, Spezifität, positiver prädiktiver Wert (PPV) und negativer prädiktiver Wert (NPV) für die Notwendigkeit einer ECMO waren $76,9 \%, 72,7 \%, 62,5 \%$ und $84,2 \%$. Für den kombinierten Endpunkt schwere PH/ECMO ergab sich eine Sensitivität von 80\%, eine Spezifität von $73,3 \%$, ein PPV von 80\% und ein NPV von $73,3 \%$.

Schlussfolgerung. Die TPV/RVET-Ratio ist ein einfach und konsistent zu gewinnender Parameter, der neben TI und DA-Fluss relevante Informationen liefert. Hoch-Risikopatienten könnten frühzeitig identifiziert und in ein ECMO-Zentrum verlegt werden. Unsere Daten bestätigen publizierte Studien, dass die TI als Stratifizierungsparameter bei Kindern mit CDH häufig ungeeignet ist.

\section{Infektiologie}

\section{FV36 \\ Molekulare Detektion von Erregern mittels SeptiFast-PCR als Entscheidungshilfe bei sehr kleinen Frühgeborenen mit Sepsis- verdacht - Resultate und Relevanz einer multizentrischen Studie}

B. Tröger', C. Härtel', J. Buer ${ }^{3}$, M. Dördelmann ${ }^{4}$, B. Hadzik', N. Hepping ${ }^{6}$, G. Hillebrand ${ }^{7}$, A. Kribs ${ }^{8}$, R. Laux 9 , D. Olbertz ${ }^{10}$, J. Siegel ${ }^{11}$, J. Steinmann ${ }^{12}$, W. Göpel ${ }^{13}$, E. Herting $^{14}$, U. Felderhoff-Müser ${ }^{15}$, A. Stein ${ }^{16}$

'Universitätsklinikum Schleswig Holstein, Kinderklinik, Lübeck, Deutschland, ${ }^{2}$ Universitätsklinikum Schleswig Holstein/Campus Lübeck, Klinik für Kinder- und Jugendmedizin, Lübeck, Deutschland, ${ }^{3}$ Institut für Medizinische Mikrobiologie der Universität, Essen, Deutschland, ${ }^{4}$ Medizinische Hochschule Hannover, Abteilung pädiatrische Pulmonologie und Neonatologie, Hannover, Deutschland, ${ }^{5}$ Med. Einricht. d. Universität Kinderklinik, Abteilung für Pädiatrische Intensivmedizin und Neonatologie, Düsseldorf, Deutschland, ${ }^{6}$ St. Marien-Hospital Bonn, Neonatologie, Bonn, Deutschland, ${ }^{7}$ Kreiskrankenhaus, Kinderabteilung, Itzehoe, Deutschland, ${ }^{8}$ Universitätskinderklinik Köln, Neonatologie und Pädiatrische Intensivmedizin, Köln, Deutschland, ${ }^{9} \mathrm{AK}$ Barmbek, Neonatologie, Hamburg, Deutschland, ${ }^{10} \mathrm{Klini}-$ kum Südstadt Rostock, Abteilung für Neonatologie und Neonatologische Intensivmedizin/Kinderschlafmedizin, Rostock, Deutschland, "Kinderkrankenhaus auf der Bult, Neonatologie, Pädiatrie I, Hannover, Deutschland, ${ }^{12}$ Universitätsklinik Essen, Medizinische Mikrobiologie, Essen, Deutschland, ${ }^{13}$ Universitätsklinikum Schleswig-Holstein, Klinik für Kinder- und Jugendmedizin, Lübeck, Deutschland, ${ }^{14}$ Universitätsklinikum Schleswig-Holstein Campus Lübeck, Direktor der Klinik für Kinder- und Jugendmedizin, Lübeck, Deutschland, ${ }^{15}$ Universitätsklinikum Essen, Direktorin der Klinik für Kinderheilkunde I, Zentrum für Kinder- und Jugendmedizin, Essen, Deutschland, ${ }^{16} \mathrm{Klinik}$ für Kinder und Jugendmedizin der Universität, Klinik für Kinderheilkunde I, Essen, Deutschland

Einleitung. 30\% der VLBW-Frühgeborenen, die im GNN geboren werden, erleiden während des stationären Aufenthalts eine klinische Sepsis. 95\% dieser Frühgeborenen werden mindestens einmal mit Antibiotika behandelt. Bei ca. 10\% der Frühgeborenen wird eine Sepsis mit Erregernachweis in der Blutkultur - als Goldstandard der Diagnostik mit entsprechenden Einschränkungen - bestätigt. Inwieweit kulturunabhängige Verfahren (z. B. Multiplex-PCRs für häufige Sepsiserreger, SeptiFast $\left.{ }^{\star}\right)$ die Diagnostik der Sepsis bei Frühgeborenen verbessern können bzw. eine klinische Entscheidungshilfe darstellen, ist bislang nicht hinreichend geklärt.
Methoden. In dieser prospektiven, multizentrischen GNN-Begleitstudie wurde bei $n=147$ Frühgeborenen mit $n=232$ klinischen Verdachtsepisoden auf eine late onset Sepsis (LOS) neben der Standarddiagnostik eine Multiplex-PCR aus $100 \mu \mathrm{l}$ EDTA-Vollblut durchgeführt. Dabei wurde der LightCycler SeptiFast MGRADE Test (Roche Diagnostics, Penzberg, Germany) eingesetzt. Dieser ermöglicht die Detektion von DNA der 26 häufigsten Sepsiserreger (Bakterien und Pilze). Neben klinischen und laborchemischen Kriterien wurde zudem die Bedeutung des PCR-Ergebnisses für die Wahl der Antibiotika und Dauer der Therapie dokumentiert.

Ergebnisse. In 50\% der Episoden gelang der molekulare Nachweis von Erreger-DNA mittels SeptiFast, während in 25\% der Episoden Erreger mittels Blutkultur detektiert werden konnten. Dabei war das Ergebnis des SeptiFast-Tests und der Blutkultur in 54,3\% übereinstimmend. Die Ergebnisse lagen bei SeptiFast an Wochentagen nach $24 \mathrm{~h}$ vor, während die Blutkulturergebnisse frühestens nach $48 \mathrm{~h}$ verfügbar waren. Die Mehrzahl der mittels SeptiFast detektierten Erreger stellten S. epidermidis (31\%) dar. In <3\% der Fälle fanden sich im Septifast-Test Enterococcus faecalis und Enterobacter cloacae, E. coli, S. aureus, Klebsiellen und Streptokokken. In der Blutkultur gelang in $14 \%$ der Nachweis von S. epidermidis. In unter $3 \%$ zeigten sich E. coli, S. aureus, S. haemolyticus, Enterococcus faecalis, Corynebacterien, Bifidobacterien und S. capitis in der Blutkultur. In 26 Episoden (12\%) wurde trotz positivem SeptiFast-Ergebnis die Diagnose Sepsis revidiert. In 16\% der Episoden hatte das SeptiFast-Ergebnis einen Einfluss auf die Wahl der Antiinfektiva, in 21\% auf die Dauer der antimikrobiellen Therapie.

Schlussfolgerung. Das SeptiFast-Ergebnis war in etwa einem Drittel der Verdachtsepisoden als Entscheidungshilfe bedeutsam. Der wesentliche Vorteil von SeptiFast gegenüber der Blutkultur liegt im schnelleren Erregernachweis aus Mikroblutproben. Als Nachteile gegenüber der Blutkultur müssen die relativ hohen Kosten, das limitierte Erregerspektrum, das höhere Kontaminationsrisiko und die fehlende Resistenztestung erwähnt werden. Daher kann die Multiplex-PCR im Wesentlichen als Add-on Diagnostik im Rahmen schwerer Infektionen dienen, z. B. bei Frühgeborenen, die unter Antibiotikatherapie keine klinische Besserung zeigen.

\section{FV37}

Fakultatives Infektions-Screening in der Schwangerschaft und Frühgeburtlichkeit: Daten aus der SNiP-Studie

\section{A. Lange', J. Olthoff', T. Ittermann ${ }^{2}$, R. Thyrian ${ }^{2}$, W. Hoffmann ${ }^{2}$, M. Heckmann ${ }^{3}$}

'Ernst-Moritz-Arndt Universität, Kinderklinik, Greifswald, Deutschland, ${ }^{2}$ Community Medicine, Universität Greifswald, Greifswald, Deutschland, ${ }^{3}$ Klinik und Poliklinik für Kinder und Jugendmedizin, Neonatologie u. Päd. Intensivmedizin, Greifswald, Deutschland

Einleitung. Infektionen in der Schwangerschaft stellen ein hohes Risiko für Mutter und Kind dar. Systemische mütterliche Infektionen können zu Abort, Tod- oder Frühgeburt führen. Die Mutterschaftsrichtlinien beinhalten Screeninguntersuchungen zu Röteln, Lues und Chlamydien-Antigen bei der Erstuntersuchung und Hepatitis B ab der 32 SSW. Dagegen werden Untersuchungen zu Infektionen mit B-Streptokokken (GBS) und Mykoplasmen nur bei Indikation oder als privat zu zahlende Gesundheitsleistung (IGeLeistung) angeboten.

Fragestellung. Wie unterscheidet sich die Screeninginzidenz von gesetzlich vorgeschriebenen und fakultativen Infektionsuntersuchungen in der Schwangerschaft? Liegt in dem fakultativen GBS- und Mykoplasmen-Screening die Gefahr potenziell vermeidbarer infektionsassoziierter Komplikationen.

Material und Methoden. Im Rahmen des populationsbasierten Survey of Neonates in Pommerania (SNiP-Study) wurden zwischen 1/2003 und 11/2008 bei 5269 Müttern und ihrer Neugeborenen Daten zu Chlamydien-, HBsAg, Röteln, Lues, Mykoplasmen/Ureaplasmen und GBS-Untersuchungen erhoben. Die Frühgeborenenrate $(\mathrm{GA}<37 \mathrm{SSW})$ lag bei $\mathrm{n}=653 / 5269(12,4 \%)$.

Ergebnisse. Im Untersuchungszeitraum fanden sich bei $n=229 / 4803(4,8 \%)$ Schwangeren ein positiver Chlamydienabstrich, $\mathrm{n}=28 / 5064(0,53 \%)$ waren HBsAg positiv, bei $n=4415 / 5268(83,8 \%)$ lag eine Rötelnimmunität 
vor (92,2\% gescreent) und ein Lues-Suchtest erfolgte bei $n=4990 / 5269$ (94,7\%). Ein fakultatives Screening auf Mykoplasmen/Ureaplasmen (positiv 111/508) wurde bei 9,64\% und auf GBS (positiv $n=105 / 513$ ) bei 9,75 der Schwangeren durchgeführt. Getestet wurden $16,5 \%$ der FG vs.8,8\% der Termingeborenen(TG). Ein positiver materialer Nachweis von Chlamydien zeigte sich bei $n=40 / 108$ (37\%)FG vs. $n=189 / 437$ (43\%) TG, von Mykoplasmen/Ureaplasmen bei $n=36 / 111$ (33\%) FG vs. $n=75 / 437 \quad(17 \%)$ TG ( $\mathrm{p}<0,01)$ und von GBS bei $\mathrm{n}=25 / 110$ (23\%) FGvs. $\mathrm{n}=8 \mathrm{o} / 437$ (18\%) TG $(\mathrm{p}<\mathrm{o}, 01)$. Bei den Neugeborenen wurden $\mathrm{n}=2$ Chlamydienkonjunktividen (ICD. A 74.0), $n=1$ Chlamydieninfektion (ICD A74.9), n=4GBS-Pneumonien (ICD P23.3), $n=1$ GBS Infektion (ICD B95.1) und $n=1$ Mykoplasmenpneumonie dokumentiert, sowie $n=182$ Infektionen die für die Perinatalperiode spezifisch sind (ICD P35-39).

Schlussfolgerung. In unserer populationsbasierten Untersuchung wurde ein fakultatives Mykoplasmen- oder GBS-Screening nur bei jeder zehnten Frau durchgeführt während die Screening-Inzidenz der gesetzlich vorgeschriebenen Infektionen bei $>90 \%$ lag. Bei Frühgeborenen lag häufiger ein positiver maternaler Mykoplasmen- und GBS-Nachweis vor als bei Geburt zum Termin. Auch wenn die fakultativen Untersuchungen häufiger bei Schwangeren mit FG durchgeführt wurden, wurden über $80 \%$ der Schwangeren mit FG nicht getestet. Weitere Untersuchungen müssen klären, ob ein generelles Screening auf Mykoplasmen und GBS die Frühgeborenenrate und die damit verbundenen Komplikationen zu senken vermag.

\section{FV38}

Wirksamkeit und Machbarkeit der CMV-Inaktivierung durch Kurzzeitpasteurisierung von Muttermilch auf neonatologischen Intensivstationen

\section{S. Bapistella', K. Hamprecht' ${ }^{2}$, W. Thomas' ${ }^{3}$, C. Speer', C. Poets', R. Goelz'}

'Eberhard-Karls-Universität Universitätsklinik für Kinderheilkunde und Jugendmedizin, Neonatologie, Tübingen, Deutschland, ${ }^{2}$ Universitätsklinikum Tübingen, Konsiliarlabor für kongenitale Virusinfektionen, Tübingen, Deutschland, ${ }^{3}$ Mutterhaus d. Borromäerinnen, Kinderklinik, Trier, Deutschland, ${ }^{4}$ Direktor der Universitäts-Kinderklinik und Poliklinik, Würzburg, Deutschland

Hintergrund und Fragestellung. In einer prospektiven Studie wurde untersucht, ob durch Kurzzeitpasteurisierung der Muttermilch (MM) die postnatale laktogene CMV-Infektion von Frühgeborenen (FG) verhindert werden kann.

Material und Methoden. In zwei neonatologischen Zentren wurden prospektiv vom 1.6.2010 bis 31.1.2012 FG $<1500$ g Geburtsgewicht oder $<32$ Schwangerschaftswochen eingeschlossen, die mit MM ernährt wurden und deren Mütter CMV-IgG-positiv waren. Eine kongenitale CMV-Infektion wurde ausgeschlossen [Urin und Speichel CMV negativ in PCR und Mikrokultur (MIK)]. Wöchentlich wurde MM und zweiwöchentlich Urin auf CMV getestet (PCR und MIK). Zielgröße war der letzte CMVUrinbefund (PCR und MIK) des FG vor Entlassung. Die MM wurde bei allen FG ab Tag 4 mit einem für diesen Zweck konstruierten Inaktivator kurzzeitpasteurisiert $\left(62^{\circ} \mathrm{C}, 5 \mathrm{~s}\right.$ lang). Die Pasteurisierung wurde beendet, wenn die MIK aus der MM zweimal hintereinander CMV-negativ, oder wenn die Entlassung des FG in den nächsten 7 Tagen zu erwarten war. Im einen Zentrum wurde die MM zentral in der Milchküche von einer Person pasteurisiert, im anderen dezentral von der jeweils betreuenden Pflegekraft.

Ergebnisse. Es konnten insgesamt 110 FG in die Auswertung eingeschlossen werden. Bei 107 (97,3\%) war die letzte CMV Mikrokultur des Urins negativ, und somit eine Infektion ausgeschlossen. Bei 3 FG $(2,7 \%)$ war die Urinkultur CMV-positiv und bewies damit eine postnatale Infektion. Bei 2 FG wurden einzelne MM-Portionen akzidentell nicht pasteurisiert, beim dritten FG ist die CMV-Quelle unklar, da in der Phase der Virolaktie die Pasteurisierung lückenlos dokumentiert war. Alle Transmissionen fanden im Zentrum mit dezentraler Pasteurisierung statt.

Schlussfolgerung. Die CMV-Inaktivierung erscheint wirksam, denn die Transmissionsrate konnte im Vergleich zu einer publizierten Kohorte von $23 \%$ [bei Ernährung mit roher MM (1)] auf 2,7\% gesenkt werden.
Sie ist im Klinikalltag auch machbar, allerdings ist die Inaktivierung an zentraler Stelle durch eine Person offenbar weniger fehleranfällig. Die Studie wurde unterstützt von: MWFK Baden Württemberg, Max-Biedermann-Institut.

1. Hamprecht $\mathrm{K}$ et al (2001) Lancet

\section{FV39}

Risikofaktoren für Late-onset-Sepsis bei VLBW-Frühgeborenen $<32$ Schwangerschaftswochen - Daten des Deutschen Frühgeborenennetzwerks

K. Hanke', B. Tröger ${ }^{2}$, A. Stein ${ }^{3}$, C. Gille ${ }^{4}$, T. Orlikowsky', C. Wieg ', E. Herting', W. Göpel ${ }^{2}$, C. Härtel'

'Universitätsklinikum Schleswig Holstein, Campus Lübeck, Klinik für Kinder-und Jugendmedizin, Lübeck, Deutschland, ${ }^{2}$ Universitätsklinikum Schleswig-Holstein, Klinik für Kinder- und Jugendmedizin, Lübeck, Deutschland, ${ }^{3}$ Klinik für Kinder und Jugendmedizin der Universität, Klinik für Kinderheilkunde I, Essen, Deutschland, ${ }^{4}$ Eberhard-Karls-Universität Universitätsklinik für Kinderheilkunde und Jugendmedizin, Neonatologie, Tübingen, Deutschland, ${ }^{5}$ Universitätsklinik für Kinder- und Jugendmedizin, Sektion für Neonatologie, Aachen, Deutschland, ${ }^{6}$ Klinikum Aschaffenburg, Neonatologie und pädiatrische Intensivmedizin, Aschaffenburg, Deutschland, ${ }^{7}$ Universitätsklinikum Schleswig-Holstein Campus Lübeck, Direktor der Klinik für Kinder- und Jugendmedizin, Lübeck, Deutschland

Fragestellung. Viele endogene und exogene Einflussfaktoren tragen zum hohen Risiko von VLBW-Frühgeborenen für Late-onset-Infektionen bei. Gegenwärtig wird kontrovers diskutiert, ob eine frühe Infektion $(<72 \mathrm{~h})$ das Risiko für eine Late-onset-Sepsis (LOS) erhöht oder durch „Training“ der Immunantwort einen protektiven Wert hat.

Material und Methoden. In unsere Analyse wurden 6817 VLBW-Frühgeborene (davon 862 mit LOS) mit einem Geburtsgewicht $<1500$ g und einem Gestationsalter $<32$ SSW aus dem Deutschen Frühgeborenennetzwerk (Geburtsjahrgänge 2009-2013) einbezogen. In einer multivariablen logistischen Regressionsanalyse wurden Einflussfaktoren auf die Entwicklung einer LOS mit positivem Erregernachweis in der Blutkultur untersucht (kovariate Faktoren: Gestationsalter, Geburtsgewicht, Geschlecht, antenatale Steroide, antenatale Antibiotika, Amnioninfektionssyndrom als Geburtsursache, frühe klinische Sepsis $<72 \mathrm{~h}$ ohne Erregernachweis, Early-onset-Sepsis mit Erregernachweis in der Blutkultur, ZVK, tracheale Beatmung).

Ergebnisse. Neben bekannten Risikofaktoren wie Gestationsalter $(\mathrm{OR}=0,83,95 \% \mathrm{CI} 0,78-0,89 ; \mathrm{p}<0,001)$ und invasive Beatmung $(\mathrm{OR}=1,52$, 95\% CI 1,25-1,84; $\mathrm{p}<0,001)$ trugen Kinder mit EOS ein deutlich erhöhtes LOS-Risiko (OR=15,60, 95\% CI 10,22-23,92; $\mathrm{p}<0,001)$. Diejenigen Kinder, die eine frühe, ausschließlich klinische Infektion hatten, waren unabhängig von anderen Risikofaktoren vor LOS geschützt $(\mathrm{OR}=0,54,95 \%$ $\mathrm{CI} 0,42-0,70 ; \mathrm{p}<0,001)$. In einer Subgruppe von Frühgeborenen mit Informationen zur stationären Ernährung ( $\mathrm{n}=2864$, davon 341 LOS) hatte Muttermilchernährung keinen signifikanten Einfluss auf das LOS-Risiko $(\mathrm{OR}=0,82,95 \% \mathrm{CI}$ o,62-1,09; $\mathrm{p}=0,18)$.

Schlussfolgerung. Die frühe klinische Infektion mit nachfolgender Antibiotikatherapie scheint in der untersuchten Kohorte das LOS-Risiko zu beeinflussen. Zukünftige Studien müssen diese epidemiologische Hypothese anhand von Modellen zur Ontogenese des frühkindlichen Immunsystems und dessen Modulation (z. B. „trainierte Immunität“) prüfen. 


\section{FV40}

Entwicklung von immunmodulatorischen Strategien im Amnioninfektionsmodell für Frühgeborene

\section{H. Hudalla', J. Pöschl', K. Buschmann', D. Frommhold" \\ 'Universitätskinderklinik Heidelberg, Klinik Neonatologie, Heidelberg, Deutschland}

Fragestellung. Gerade bei extrem Frühgeborenen ist das Amnioninfektionssyndrom (AIS) durch eine systemische fetale Immunantwort (FIRS - Fetal Inflammatory Response Syndrome) mit hoher Morbidität und Mortalität assoziiert. Grundlegende Mechanismen und potenzielle Behandlungsansätze werden dringend benötigt. Unter Nutzung eines neuen Amnioninfektionsmodelles können immunmodulatorische Strategien untersucht werden.

Material und Methoden. Zur Induktion einer FIRS ähnlichen Amnioninfektion wurde schwangeren Mäuse zwischen Gestationsstadium E13 und E18 (entsprechend einem Gestationsalter von 24-36 Wochen beim Menschen) $2 \times 0,25 \mu \mathrm{g} / \mathrm{g}$ LPS intraperitoneal innerhalb von 16 Stunden verabreicht. Nach Anästhesie und Uterotomie wurde die Leukozytenrekrutierung mittels intravitaler Immunfluoreszenzmikroskopie in vivo gemessen. Als transgene Mauslinien dienten LysEGFP- und zur Untersuchung der ontogenetischen Regulierung des CD95-Systems und dessen Rolle in der unreifen Immunantwort LysYFP-CD95-/--Mäuse. Organe der Feten sowie die Plazenta wurden für die histologische Auswertung der Organinfiltration verwendet.

Ergebnisse. In Übereinstimmung mit unseren Voruntersuchungen reifen die Leukozytenrekrutierungsvorgänge im AIS-Modell im beobachteten Zeitraum der fetalen Entwicklung graduell aus, was sich in der histologisch beschriebenen Leukozyteninfiltration fetaler Organe (Lunge, Gehirn, Niere) widerspiegelt. Eine relevante Chorioamnionitis als histologisches Korrelat eines AIS zeigte sich in allen Entwicklungsstadien. Erste Ergebnisse deuten darauf hin, dass das CD95/Apo/Fas-System in frühen Entwicklungsstadien einen hohen regulatorischen, proinflammatorischen Effekt hat. Knockout des CD95-Receptors auf fetalen Leukozyten hemmt Rekrutierungsvorgänge über die bestehende Unreife hinaus. Ferner gelang eine erste Testung immunologisch wirksamer Substanzen. Diskussion. Die Schwierigkeit der Immunität des Fetus bzw. des extrem Frühgeborenen besteht in sich ständig wandelnden, ontogenetischen Expressionsmustern pro- und anti-inflammatorischer Faktoren. Ein Modell zur Untersuchung immunologischer Prozesse in vivo in allen Entwicklungsstadien ist folglich von hoher wissenschaftlicher Bedeutung. Wir zeigen hier an unserem Model erste Ansätze zur Untersuchung dieser ontogenetischen Expressionen am Beispiel des CD95Systems. Ferner ist an diesem Modell eine gute präklinische Testung bekannter und neuer Substanzen zur Immunmodulation (wie z. B. Steroide, Coffein, Macrolide) möglich. Dies erleichtert auch die gezielte Entwicklung vielversprechender therapeutischer Optionen zur Behandlung immunologischer Störungen bei Frühgeborenen.
Schlussfolgerung. Unser Amnioninfektionsmodel eignet sich zur Untersuchung ontogenetisch regulierter inflammatorischer Faktoren. Die Wirksamkeit potentieller Immunmodulatoren kann dadurch gestationsalterspezifisch untersucht werden.

\section{FV41}

Risikoprofile für invasive Pilzinfektionen und empirischen Einsatz von Antimykotika bei sehr kleinen Frühgeborenen mit einem Geburtsgewicht unter $1500 \mathrm{~g}$

P. Paul', A. Hartz', J. Pagel', F. Pulzer', A. Simon', A. Kribs', A. Stein', M. Vochem", A. Müller', C. Wieg ${ }^{8}$, E. Herting', W. Göpel', C. Härtel'

'Universitätsklinikum Schleswig-Holstein, Klinik für Kinder- und Jugendmedizin, Lübeck, Deutschland, ${ }^{2}$ Universitätsklinikum Leipzig, Abteilung Neonatologie, Leipzig, Deutschland, ${ }^{3}$ Universitätsklinikum des Saarlandes, Klinik für Pädiatrische Onkologie und Hämatologie, Homburg, Deutschland, ${ }^{4}$ Universitätskinderklinik Köln, Neonatologie und Pädiatrische Intensivmedizin, Köln, Deutschland, ${ }^{5}$ Klinik für Kinder und Jugendmedizin der Universität, Klinik für Kinderheilkunde I, Essen, Deutschland, ${ }^{6}$ Olgahospital, Neonatologie, Stuttgart, Deutschland, ${ }^{7}$ Zentrum für Kinderheilkunde am Universitätsklinikum Bonn, Neonatologie, Bonn, Deutschland, ${ }^{8}$ Klinikum Aschaffenburg, Neonatologie und pädiatrische Intensivmedizin, Aschaffenburg, Deutschland

Hintergrund. Sehr kleine Frühgeborene mit einem Geburtsgewicht $<1500 \mathrm{~g}$ (,very low birth weight“, VLBW) unterliegen einer ausgeprägten Vulnerabilität für Infektionen, die mit intravenösen Antibiotika und zudem häufig mit systemischen Antimykotika behandelt werden. Ziel unserer Untersuchung war die Charakterisierung der klinischen Einflussgrößen für invasive Pilzinfektionen und Erfassung der Behandlung mit Antimykotika in einer großen Kohorte von VLBW-Frühgeborenen. Methoden. Datenanalyse von n=6102 VLBW-Frühgeborenen des Deutschen Frühgeborenennetzwerkes (GNN) der Jahre 2009 bis 2012.

Ergebnisse. Während systemische Antimykotika deutlich häufiger eingesetzt wurden $(n=214$ Fluconazol, $n=73$ Amphothericin $B, n=14$ Voriconazol, $n=13$ Caspofungin), zeigten nur 26/6102 (o,4\%) der VLBWFrügeborenen eine mittels Blutkultur bestätigte Candida-Sepsis. Als patientenbezogene Risikofaktoren für die Pilzinfektion/Behandlung mit Antimykotika wurden Gestationsalter und Geburtsgewicht determiniert. Besonders vulnerabel war die Subgruppe der $<27$ SSW Frühgeborenen, bei der mit intravenösen Antimykotika behandelte Kinder $(11,4 \%, 225 / 1969)$ jünger und leichter als die unbehandelten Kinder waren $(24,7$ vs. 25,4 SSW, $\mathrm{p}<0,001 ; 630$ g vs. $736 \mathrm{~g}, \mathrm{p}<0,001)$. Die behandlungsbezogenen Risikofaktoren beinhalteten ZVK, Dauer des stationären Aufenthalts, Vorerkrankung, Blutkultur-positive Sepsis, Gabe von Cephalosporinen und Carbapenemen. Zudem zeigte sich, dass die Pilzinfektion/Behandlung mit Antimykotika mit signifikanter Langzeitmorbidität assoziiert ist (Tab. FV41).

Tab. FV41 Outcome von Frühgeborenen <27 SSW in Abhängigkeit von der Notwendigkeit intravenöser Antimykotika bzw. Nachweis einer invasiven Candida-Infektion

\begin{tabular}{|lllll} 
& $\begin{array}{l}\text { Keine i.v. Antimykotika } \\
\mathbf{n}=\mathbf{1 5 9 3}\end{array}$ & $\begin{array}{l}\text { i.v. Antimykotika } \\
\mathbf{n}=\mathbf{2 2 8}\end{array}$ & $\begin{array}{l}\text { Keine Candida-Infektion } \\
\mathbf{n}=1730\end{array}$ & $\begin{array}{l}\text { Candida-Infektion } \\
\mathbf{n}=19\end{array}$ \\
\hline PVL (\%) & 4,8 & 5,9 & 4,8 & 5,6 \\
\hline BPD (\%) & 31,3 & $49,8(\mathrm{p}<0,001)$ & 33,5 & 33,8 \\
\hline Tod (\%) & 9,5 & 11,4 & 9,1 & $26,3(\mathrm{p}=0,01)$ \\
\hline Auffälliges Hörscreening (\%) & 13,9 & $23,2(\mathrm{p}<0,001)$ & 14,8 & $45,5(\mathrm{p}=0,005)$ \\
\hline Brille (F/U, \%) & 13,1 & $28,6(\mathrm{p}=0,007)$ & 14,8 & n.a. \\
\hline Freies Laufen (F/U, \%) & 90,3 & $75,0(\mathrm{p}=0,001)$ & 88,7 & n.a. \\
\hline MDI (F/U; Median, IQR) & $96(80-106)$ & $78(64-96)$ & $94(78-106)$ & n.a. \\
\hline P-Wen & &
\end{tabular}

p-Werte Fisher's exact test bzw. Mann-Whitney U-Test; PVL periventrikuläre Leukomalazie, BPD bronchopulmonale Dysplasie, F/U Follow-up im Alter von $21-24$ Monaten, MDI Median Developmental Index, IQR Interquartile Range, n.a. nicht angegeben. 
Diskussion. Die invasive Pilzinfektion ist ein seltenes Ereignis bei VLBW-Frühgeborenen in der erfassten GNN-Kohorte. Eine erhebliche Anzahl von Kindern, insbesondere $<27$ SSW, wird empirisch mit Antimykotika behandelt. In Anbetracht des ungünstigen Risikoprofils der behandelten Kinder und der unzulänglichen Datenlage zur Pharmakodynamik müssen weitere Studien den Nutzen der Antimykotika prüfen.

\section{Neonatologie: Ernährung}

\section{FV42}

\section{Validation of NIR milk analyzer for pasteurized and native milk}

G. Fusch', A. Choi', C. Kwan', D. Choi', S. Huang', N. Rochow', C. Fusch'

'Pediatrics, McMaster University, Hamilton, Ontario, Canada

Background. Reliable milk analysis is a prerequisite to implement the concept of target fortification in the NICU. We recently showed that this concept is feasible (Rochow 2013) but that IR milk analyzers need to be evaluated. We proposed equations to improve accuracy for fat and protein analysis that need to be validated (Fusch 2014). Additionally, we study the impact of pasteurization on milk analysis.

Objective. 1) To validate published correction algorithms for fresh or frozen breast milk, 2) to assess the impact of pasteurization on IR analysis (Unity SpectraStar) and chemical analysis

Methods. Pooled breast milk samples $(\mathrm{N}=50)$ were divided into control and Holder pasteurized. 1) validation (only fat and protein): correlation analysis of 20 unpasteurized and 10 corresponding pasteurized samples measured with IR analyzer and chemical reference methods. 2) For pasteurization (F, P and lactose): correlation analysis of unpasteurized and pasteurized samples measured with (1) IR analyzer ( $\mathrm{n}=50$ each) and (2) chemical reference methods $(\mathrm{n}=10)$.

Results. Validation shows reliability of proposed correction algorithms (Abb. FV42.1). Pasteurization has no influence on IR and chemical method readouts (Abb. FV42.2).

Conclusions. Published algorithms can be used to correct for fat and protein readouts of the IR analyzer. Pasteurized milk can be rapidly assessed and target fortified which is important to identify low protein donor milk.

1. Fusch C et al (2014) Clin Nutr. doi: 10.1016/j.clnu.2014.05.005

2. Rochow N et al (2013) J Pediatr 163(4):1001-1007

\section{Hygiene}

\section{FV43 \\ Ergebnisse des mikrobiellen Kolonisationsscreenings auf einer neonatologischen Intensivstation (2014)}

\section{F. Pulzer', N. Lippmann'², A. Behne', A. Bläser', M. Knüpfer', C. Gebauer', L.} Wronski-Löffelbein', T. Wallborn', U. Thome'

'Universitätskinderklinik, Neonatologie, Leipzig, Deutschland, ${ }^{2}$ Uniklinikum Leipzig, Institut für medizinische Mikrobiologie und Infektionsepidemiologie, Leipzig, Deutschland, ${ }^{3}$ Institut für Hygiene/ Krankenhaushygiene, Leipzig, Deutschland

Einleitung. Die Inzidenz an Infektionsausbrüchen durch multiresistente gramnegative Erreger (MRGN) scheint auch bei Früh- und Neugeborenen zu steigen. Wir berichten über unsere Erfahrungen mit der Umsetzung des mikrobiologischen Kolonisationsscreenings, welches durch die Kommission für Krankenhaushygiene und Infektionsprävention (KRINKO) empfohlen wird.

Methodik. Während einer 12-monatigen Beobachtungszeit wurden 733 Patienten auf unsere neonatologische Intensivstation übernommen. Die Ergebnisse der bei stationärer Aufnahme und in Folge wöchentlich durchgeführten Rachen- und Stuhlabstriche zur Detektion von MRGN wurden analysiert.

Ergebnisse. Bei den 733 stationär betreuten Patienten (23.2-42.4 SSW) wurden 2967 Rachen- und Stuhlabstriche sowie 170 Blutkulturen (BK) gewonnen. Hierbei gelang der Nachweis von MRGN bei 65 Patienten (Rachen und Stuhl). Das Aufnahmescreening war in 7 Fällen positiv. Wir konnten eine Häufung an Besiedelungen mit 2MRGN Acinetobacter baumannii (14 Patienten) und 2MRGN Enterobacter cloacae (15 Patienten) sowie 3 MRGN E. coli bei 24 Patienten feststellen. In 4 Fällen lag eine Doppelbesiedelung mit diesen Erregern vor, in einem Fall eine positive BK durch 3 MRGN E. coli. 23 von 24 Patienten wiesen eine sehr hohe genomische Ähnlichkeit der 3 MRGN E. coli Isolate auf. Des Weiteren wurden bei einzelnen Patienten auch 2- bzw. 3MRGN Klebsiella spp., VRE Enterococcus faecalis, MBL bzw. 4MRGN E. coli sowie Serratia marcescens und Citrobacter freundii (jeweils 2 MRGN) nachgewiesen. MRSA positive Abstriche wurden nicht gefunden. Das empirische Antibiotikaregime wurde an das jeweilige Resistenzmuster angepasst. Umgebungsuntersuchungen waren nicht wegweisend. Schwere Erkrankungen bzw. Todesfälle in Zusammenhang mit einer Besiedelung/Infektion wurden nicht beobachtet.

Schlussfolgerung. Durch das mikrobielle Routinescreening und die Erregertypisierung konnten eine Häufung an Besiedelungen mehrerer Patienten mit MRGN beobachtet und spezielle hygienische Maßnahmen ergriffen werden. Konsequente Screeninguntersuchungen sowie Präventionsmaßnahmen und eine Anpassung der empirischen Antibiotikatherapie sind wichtig, um Infektionsausbrüche zu verhindern.

\section{FV44}

Besiedelung von beatmeten pädiatrischen Intensivpatienten mit multiresistenten Enterobakterien: epidemiologische Daten und Risikofaktoren

L. Stoll', H. Renk', F. Neunhoeffer', M. Kumpf', M. Hofbeck', D. Hart ${ }^{2}$

'Universitätsklinik für Kinderheilkunde und Jugendmedizin/EberhardKarls-Universität/Kinderheilkunde II (Kardiologie, Pulmologie, Intensivmedizin), Tübingen, Deutschland, ${ }^{2}$ Universitätsklinik für, Kinder- und Jugendmedizin, Tübingen, Deutschland

Hintergrund. Die Prävalenz Gram-negativer Infektionen wird für pädiatrische Intensivstationen zunehmend relevant. Multiresistente Gramnegative Erreger stellen Pflegekräfte, Ärzte und Krankenhausökonomie vor hygienische, therapeutische und finanzielle Probleme.

Fragestellung. Ziel dieser Studie war es, die Besiedelung mit Enterobakterien bei pädiatrischen Intensivpatienten in den Jahren 2005-2013 zu ermitteln. Zusätzlich sollten prädiktive Faktoren für eine Kolonisation von beatmeten Patienten mit multiresistenten Enterobakterien bestimmt werden.

Methoden. Von 2005 bis 2013 wurde eine retrospektive Single-centerAnalyse der Enterobakterien Isolate aller pädiatrischen Intensivpatienten (o-18 Jahre) durchgeführt. Epidemiologische und klinische Daten wurden aus der mikrobiologischen Datenbank der Universitätsklinik Tübingen, sowie aus den elektronischen Krankenakten der intubierten Kinder mit Nachweis von Enterobakterien im Trachealsekret, erhoben. Relevante Faktoren für die Besiedelung mit multiresistenten Enterobakterien wurden mittels univariater und mulitvariabler Regressionsanalyse ermittelt.

Ergebnisse. Die Inzidenz der Enterobakterien-Besiedelung von pädiatrischen Intensivpatienten verdoppelte sich während des Untersuchungszeitraums von 5,6\% im Jahr 2005 auf 10,1\% im Jahr 2013. Im gleichen Zeitraum stieg der Anteil multiresistenter Gram-negativer Isolate um das Vierfache (von 0,5\% auf 2,2\%). Der größte Anteil an Enterobakterien (58\%) wurde aus Material des unteren Respirationstrakts isoliert. Die häufigste Spezies war E. coli (30.8\%), gefolgt von Enterobacter (30,1\%) und Klebsiellen (25,6\%). Während der Studienperiode wurden bei 110 intubierten pädiatrischen Intensivpatienten 156 Enterobakterien Nachweise aus Trachealsekret erbracht. 52 (33\%) dieser Isolate waren multiresistent. Als signifikante Risikofaktoren für eine Kolonisation mit multi- 

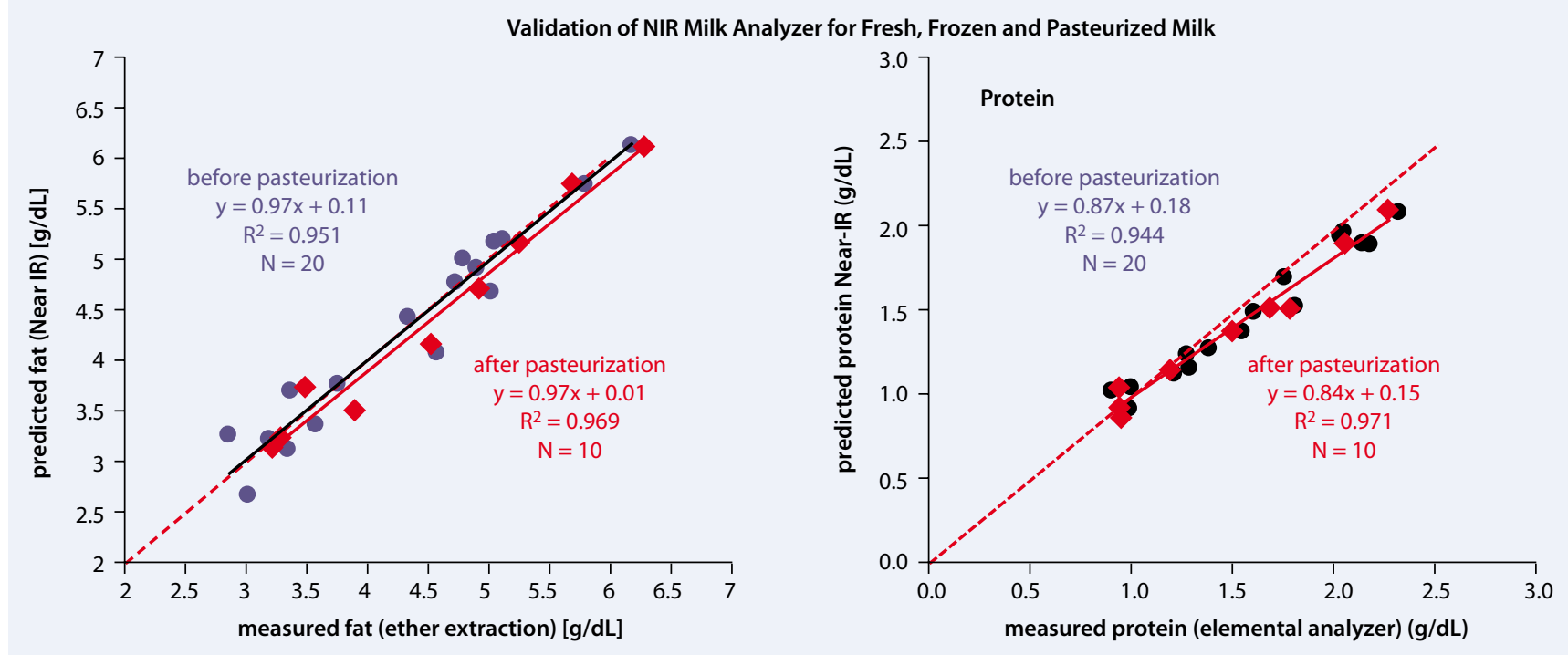

Abb. FV42.1 1

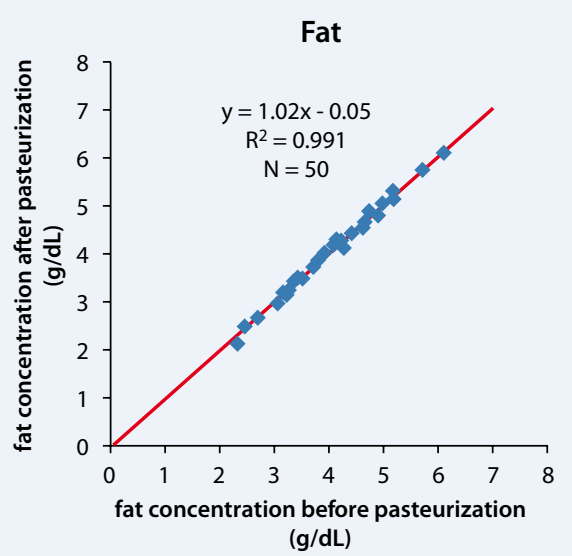

Unity SpectraStar IR Milk Analyzer
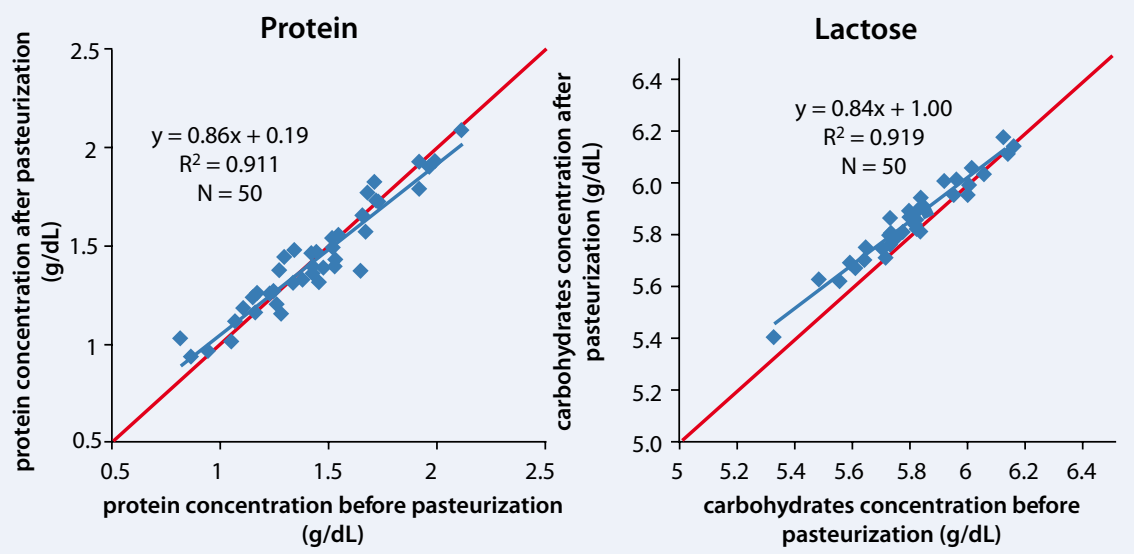

Validated Wet Lab Methods
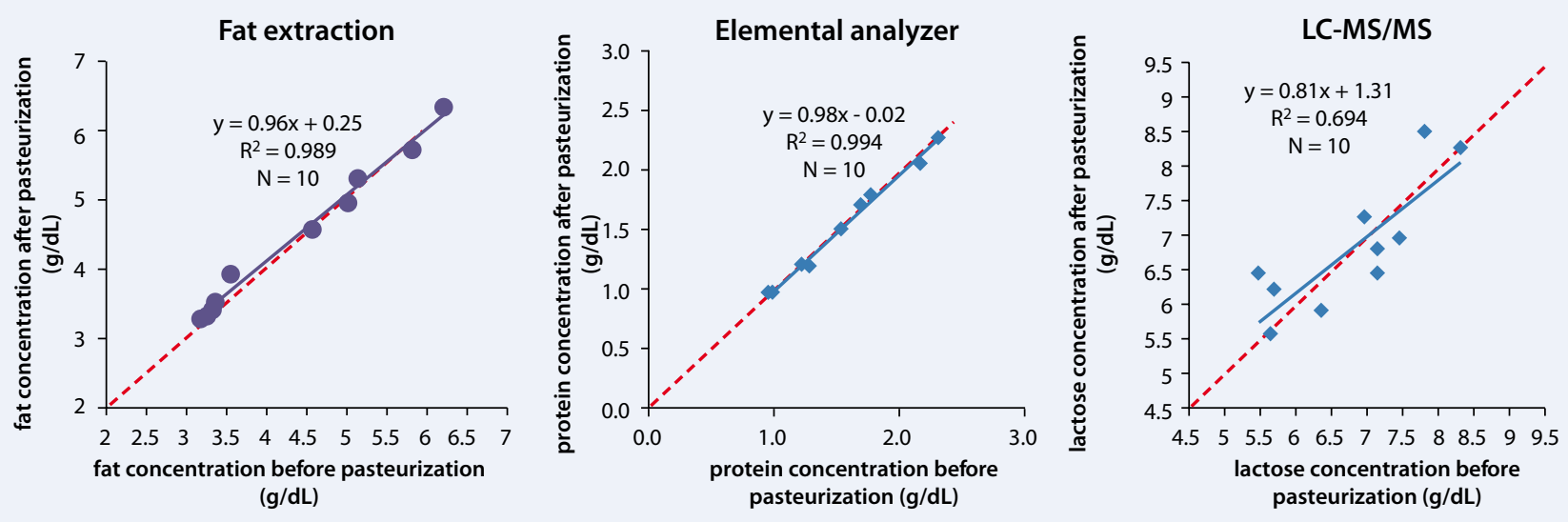

Abb. FV42.2 1 
resistenten Enterobakterien fanden wir die Besiedelung mit E. coli (OR $5,6,95 \%$ CI 2,7-11,8) und das Vorliegen einer gastrointestinalen Komorbidität (OR 2,6, 95\% CI 1,3-5,4). Schwächere Assoziationen wurden zwischen multiresistenten Enterobakterien und einem vorhandenen ZVK, den Beatmungstagen und der Dauer der antibiotischen Behandlung vor Kultur beobachtet.

Schlussfolgerung. Von 2005-2013 verdoppelte sich die Inzidenz der Enterobakterien-Besiedelung auf unserer pädiatrischen Intensivstation. Multiresistente Organismen nahmen um ein Vierfaches zu. Die Isolation von E. coli und gastrointestinale Komorbiditäten stellten sich als relevante Risikofaktoren für eine Besiedelung des unteren Respirationstraktes mit multiresistenten Erregern heraus.

\section{FV45}

\section{Auftreten und Übertragung von Enterobacter gergoviae auf einer neonatologischen Intensivstation}

\section{A. Kirste', H. Weiskopf', C. Gille', J. Liese ${ }^{1}$}

'Universitätsklinikum Tübingen, Institut für Med. Mikrobiologie und Hygiene, Tübingen, Deutschland, ${ }^{2}$ Eberhard-Karls-Universität Universitätsklinik für Kinderheilkunde und Jugendmedizin, Neonatologie, Tübingen, Deutschland

Hintergrund. Für Frühgeborene ist die Gefahr einer nosokomialen Sepsis während des stationären Aufenthaltes sehr hoch. Ausgangspunkt ist häufig das Mikrobiom der Haut und des Gastrointestinaltrakts, das wiederum stark von der patientennahen Umgebung beeinflusst wird Seit einiger Zeit wird für intensivmedizinisch behandelte Früh- und Neugeborene ein wöchentliches mikrobiologisches Screening empfohlen, um auf eine Besiedlung mit potentiell pathogenen Erregern im Fall einer Infektion schnell reagieren zu können und um Übertragungen rasch zu erkennen. Im Sommer 2014 fiel im Rahmen des Screenings in unserer Institution eine Häufung von Nachweisen von Enterobacter (E.) gergoviae bei verschiedenen Patienten auf einer neonatologischen Intensivbehandlungsstation auf. Dieser zu den Enterobacteriaceae zählende Erreger wird üblicherweise sehr selten nachgewiesen. Die Isolate zeigten keine ungewöhnlichen Resistenzen gegen die getesteten Antibiotika.

Fragestellung. Es wurde ein monoklonaler Ausbruch mit E. gergoviae vermutet und versucht, die Übertragungswege nachzuzeichnen sowie eine potentielle Quelle des Erregers zu identifizieren.

Methoden. Die mikrobiologischen Screening-Ergebnisse der Intensivbehandlungsstation (BHS) und der Intensivüberwachungsstation (ÜWS) wurden über einen Zeitraum von 4 Monaten auf das Vorliegen von E. gergoviae untersucht und mit den Patientenbelegungsdaten korreliert. Durch mehr als 40 krankenhaushygienische Umgebungsuntersuchungen wurde nach einer möglichen Keimquelle gesucht.

Ergebnisse. Während des Beobachtungszeitraums fand sich E. gergoviae bei 15 Kindern in mindestens einem Screeningabstrich, wobei bis zu 10 Kinder zeitgleich besiedelt waren. Durch Verlegung breitete sich der Erreger von der BHS auf die ÜWS aus. Neubesiedlungen fanden ganz überwiegend in Patientenzimmern mit einem bereits besiedelten Kind statt. Infektionen mit E. gergoviae wurden nicht beobachtet. Das Ausbruchsgeschehen endete mit Entlassung des letzten besiedelten $\mathrm{Pa}-$ tienten. Der einzige Nachweis von E. gergoviae in der unbelebten Umgebung erfolgte von einem patientenbezogen verwendeten Stethoskop. Alle anderen Untersuchungen insbesondere von Pflegeutensilien (Öle) und Diagnostikzubehör (Ultraschallgel) blieben negativ.

Diskussion. Die Abwesenheit einer exogenen Infektionsquelle unterstreicht die Bedeutung der kolonisierten Patienten als Erregerreservoir für Kontaktübertragungen, die im Wesentlichen durch das Personal vermittelt werden, und verdeutlicht die Wichtigkeit der Basishygienemaßnahmen auch bei nicht resistenten Keimen. Das hier beschriebene Ausbruchsgeschehen veranschaulicht das hohe Verbreitungspotential von Enterobacteriaceae insbesondere auf Intensivstationen. Beim gehäuften Auftreten ungewöhnlicher Erreger sollten sofort ein Ausbruch in Erwägung gezogen und mögliche Übertragungswege analysiert werden.

\section{FV46}

Barrierepflege bei Frühgeborenen mit einem Geburtsgewicht unter 1500 Gramm (VLBWI) und Besiedelung mit Methicillinsensiblen S. aureus (MSSA). Auswirkung auf Kolonisations- und Sepsisrate

\section{W. Lindner', H. Hummler', H. von Baum ${ }^{3}$}

'Universitätsklinikum Ulm, Klinik für Kinder- und Jugendmedizin, Sktion Neonatologie und Pädiatrische Intensivmedizin, Ulm, Deutschland, ${ }^{2}$ Klinik für Kinder- und Jugendmedizin, Universitätsklinikum Ulm, Sektion Neonatologie und Pädiatrische Intensivmedizin, Ulm, Deutschland, ${ }^{3}$ Universitätsklinikum Ulm, Institut für Medizinische Mikrobiologie und Hygiene, Ulm, Deutschland

Hintergrund. Zusätzlich zu dem bei VLBWI empfohlenen Screening auf multiresistente Erreger können Pathogene ohne Multiresistenz wie MSSA in den Screeningproben differenziert werden. Im Screeningzeitraum 7/2012 bis 3/2014 war MSSA der häufigste Erreger Blutkultur positiver nosokomialer Sepsen in einem universitären Perinatalzentrum Level III in Deutschland.

Ziel. Reduktion von MSSA Kolonisations- und Sepsisraten durch hygienische Barrieremaßnahmen.

Methodik. Intervention: Ab 3/2014 Beginn der Barrierepflege (Kittelund Handschuhpflege) von VLBWI bei Nachweis einer MSSA Kolonisation. Vergleich der Zeiträume vor (19,5 Monate) und während Intervention (10 Monate) in Bezug auf MSSA Kolonisations- und Sepsisraten. Ergebnisse. Im Zeitraum 7/2012 bis 12/2014 wurden 270 VLBWI [Reifealter 28,1 (22,3-37 SSW, Geburtsgewicht. 980 (300-1490) g, 11235 Surveillancetage] untersucht. 91 (34\%) VLBWI waren mit MSSA besiedelt. Vor Intervention gab es $10(5,6 \%)$ VLBWI mit MSSA Sepsis im Alter von 11,5 (6-36) d (Inzidenzdichte 1,27/100o Surveillancetage). Mit Beginn der Barrieremaßnahmen (9 Monate, 93 VLBWI) gab es Trends zu einer Abnahme der MSSA-Kolonisierungsrate, sowie zu späterer Kolonisierung (Tab. FV46). Bei dem Sepsis-Kolonisation-Verhältnis vor Intervention $(1 / 6,5)$ wären 4 VLBWI mit MSSA-Sepsis zu erwarten gewesen. Es trat keine MSSA-Sepsis mehr auf ( $\mathrm{p}=0,047)$.

Schlussfolgerung. Durch klinikhygienische Barrieremaßnahmen können die MSSA Kolonisations- und Infektionsraten von VLBWI reduziert werden.

\section{FV47}

Kolonisation von Frühgeborenen mit einem Geburtsgewicht $<1500 \mathrm{~g}$ (VLBWI) mit multiresistenten Erregern (MRE) in Abhängigkeit von der mütterlichen und neonatalen Antibiotikaexposition

\section{W. Lindner', F. Reister ${ }^{2}$, H. Hummler ${ }^{3}$, A. Essig ${ }^{4}$, H. von Baum ${ }^{4}$}

'Universitätsklinikum Ulm, Klinik für Kinder- und Jugendmedizin, Sektion Neonatologie und Pädiatrische Intensivmedizin, Ulm, Deutschland, ${ }^{2}$ Universitätsklinikum Ulm, Universitätsfrauenklinik, Ulm, Deutschland, ${ }^{3} \mathrm{Klinik}$ für Kinder- und Jugendmedizin, Universitätsklinikum Ulm, Sektion Neonatologie und Pädiatrische Intensivmedizin, Ulm, Deutschland, ${ }^{4}$ Universitätsklinikum Ulm, Institut für Medizinische Mikrobiologie und Hygiene, Ulm, Deutschland

Hintergrund. Mikrobiologisches MRE-Screening wird bei VLBWI seit 2012, bei Schwangeren mit drohender Frühgeburt seit 2013 empfohlen. Ziel. Untersuchung der Auswirkung von prä- und postnataler Antibiotikaexposition auf die MRE-Kolonisation von Müttern und VLBWI. Methodik. Auswertung von präpartalen mütterlichen mikrobiologischen Befunden, neonatalen Screeningdaten und prä- und postnatalen Antibiotikatagen aller VLBWI, die im Zeitraum $7 / 2012$ bis 6/2014 in einem universitären Perinatalzentrum Level III in Deutschland geboren wurden.

Ergebnisse. 206 VLBWI [Reifealter 28 (22,3-37 SSW, Geb.-Gew. 950 (300-1490) g, 9298 Surveillancetage] von 175 Müttern wurden untersucht. Bei $117 / 175$ (67\%) Müttern gab es präpartale Vaginalabstriche. 
Tab. FV46 Demographische Daten und Angaben zu MSSA-Kolonisations- und Infektionshäufigkeit vor und während Barrierepflege

bei MSSA-kolonisierten FG

\begin{tabular}{|llll}
\hline & Vor Intervention & Während Intervention & p-Wert \\
\hline N, FG & 177 & 93 & 0,98 \\
\hline Gestationsalter (SSW) & $28,3(22,3-37,0)$ & $27,9(23,1-36,0)$ & 0,74 \\
\hline Geburtsgewicht (g) & $950(310-1490)$ & $1030(300-1490)$ & \\
\hline Surveillancetage (d) & 7863 & 3372 & 0,19 \\
\hline MSSA-Kolonisation, N (\%) & $65(37)$ & $26(28)$ & 0,09 \\
\hline Alter bei Erstnachweis (d) & $14(2-68)$ & $19(3-51)$ & 0,32 \\
\hline MSSA als erster Leitkeim & $23 / 65(35)$ & $6 / 26(23)$ & 0,047 \\
\hline MSSA-Sepsis, N (\%) & $10(5,6)$ & 0 & 0,057 \\
\hline N, Sepsis/N, Kolonisation & $1 / 6,5$ & $0 / 26$ & \\
\hline
\end{tabular}

Tab. FV47 Demographische Daten und Angaben zu MRE Kolonisation und Antibiotikatagen bei VLBWI

\begin{tabular}{|llll}
\hline & MRE-Kolonisation & & p-Wert \\
\hline & Ja & Nein & \\
\hline VLBWI (N) & 28 & 178 & 0,001 \\
\hline Gestationsalter (SSW) & $25,8(23-30,4)$ & $28,4(22,3-37,0)$ & 0,002 \\
\hline Geburtsgewicht (g) & $733(450-1380)$ & $983(300-1490)$ & 0,0001 \\
\hline Surveillancetage (d) & $62(25-99)$ & $39(10-109)$ & 0,36 \\
\hline Alter bei MRE-Erstnachweis (d) & $34(1-67)$ & $150(84)$ & 0,005 \\
\hline Antibiotika gesamt, N (\%) & $26(93)$ & $5(0-80)$ & 0,57 \\
\hline Tage & $11(0-112)$ & $69(39)$ & \\
\hline Präpartal N (\%) & $13(46)$ & & 0,43 \\
& & & 0,18 \\
\hline Tage & & & 0,001 \\
\hline Postnatal N (\%) & & 0,07 \\
\hline Tage & $0(0-80)$ & $143(80)$ & 0,26 \\
\hline V. a. konnatale Infektion, N (\%) & $26(93)$ & $3(0-33)$ & 0,006 \\
\hline Tage & $7,5(0-55)$ & $126(71)$ & 0,002 \\
\hline V.a. nosokomiale Infektion, N (\%) & $25(89)$ & $3\left(0-21^{*}\right)$ & $68(38)$ \\
\hline Tage & $3(0-4)$ & $0(0-32)$ & \\
\hline *21 Tage Antibiotika bei konnataler Listerieninfektion. & $19(68)$ & & \\
\hline
\end{tabular}

8/117 (7\%) dieser Mütter und 28/206 (14\%) VLBWI waren MRE-kolonisiert (Mütter: 3MRGN, N=3; 2MRGN, N=5. VLBWI: 3MRGN, N=3; 2MRGN, $\mathrm{N}=21$; MRSA, $\mathrm{N}=4$ ). Eine vertikale Transmission fand bei $5 / 9$ VLBWI der MRE positiven Mütter statt (prä-/peripartal, N=2; im Verlauf, $\mathrm{N}=3$ ).

Präpartale Antibiotikaexposition. Es gab 1008 präpartale Antibiotikatage. 95 Feten von $82 / 175$ (47\%) Schwangeren waren an 6 (1-80) Tagen antibiotikaexponiert. 21 Schwangere erhielten präpartal Antibiotika $>14 \mathrm{~d}$ bei vorzeitigem Blasensprung. Die $8 \mathrm{MRE}$ positiven Mütter erhielten präpartal Antibiotika für 26 (o-80) Tage. Die Dauer der präpartalen Antibiotikagabe war bei den Müttern mit präpartalen Vaginalabstrichen signifikant mit der mütterlichen MRE-Kolonisation assoziiert $(\mathrm{p}=0,008)$. Mütter mit Antibiotikatherapie $>14 \mathrm{~d}$ waren öfter mit MRE kolonisiert (5/21, 23,8\%) wie Mütter mit weniger Antibiotikatagen (2/50, $4 \%),(p=0,022)$. Eine von 46 (2,2\%) Müttern ohne Antibiotikatherapie war MRE positiv.

Postnatale Antibiotikaexposition. Es gab 1412 postnatale Antibiotikatage (V. a. konnatale und nosokomiale Infektion: 595 und 817 d). Die Antibiotika-Anwendungsraten lagen unter den 25\%-Quantilen der NeoKISS
Referenzwerte. Bei V.a. konnatale Infektion erhielten 151 (73\%) VLBWI für 3 (1-21) d Antibiotika. Bei V. a. nosokomiale Infektion erhielten 87 (42\%) VLBWI für 8 (1-52) d Antibiotika. Die höchste Rate an MREKolonisation (10/30, 33\%) fand sich in der Gruppe der VLBWI, die präpartal und postnatal bei V. a. konnatale und nosokomiale Infektionen antibiotikaexponiert waren. Weitere Daten zu Antibiotikatagen und MRE-Kolonisation (Tab. FV47). Bei multivariater Analyse der signifikanten Größen (Gestationsalter, Geburtsgewicht, Surveillancedauer, Antibiotikatage: gesamt, postnatal und nosokomial) blieb nur das Gestationsalter als signifikante Einflussgröße. Es gab eine Pneumonie mit ${ }_{3}$ MRGN-E. coli.

Schlussfolgerung. Bei präpartaler Antibiotikagabe steigt das Risiko der mütterlichen MRE-Kolonisation mit vertikaler Transmission. In einer Einrichtung mit sehr niedriger Antibiotika-Anwendungsrate war die postnatale MRE-Kolonisierung der VLBWI signifikant mit dem Gestationsalter (= längere Surveillancedauer) aber nicht mit der prä- oder postnatalen Antibiotikaexposition assoziiert. Dies spricht für horizontale Transmission und unterstreicht die Bedeutung adäquater klinikhygienischer Maßnahmen bei der Betreuung von VLBWI. 


\section{FV48}

Mikrobiologische Screeningdaten zeigen typische Besiedlungsabläufe bei Früh- und Neugeborenen und eine große Variabilität im Auftreten potentiell pathogener Keime

\section{Gille', J. Arand', A. Kirste', M. Marschal', C. Poets', I. Autenrieth', J. Liese ${ }^{2}$}

'Eberhard-Karls-Universität Universitätsklinik für Kinderheilkunde und Jugendmedizin, Neonatologie, Tübingen, Deutschland, ${ }^{2}$ Universitätsklinikum Tübingen, Institut für Med. Mikrobiologie und Hygiene, Tübingen, Deutschland

Hintergrund. Das seit 2012 für neonatologische Intensivstationen wöchentlich empfohlene Besiedlungsscreening mittels Rachen- und Analabstrich soll potentiell pathogene Besiedlung einzelner Patienten ermitteln und nosokomiale Übertragungen aufdecken. Bisher ist wenig über die physiologische postnatale Besiedlung neonatologischer Intensivpatienten sowie die Auftretenshäufigkeit potentiell pathogener Keime bekannt.

Ziel. (1) Longitudinale Auswertung von Besiedlungsdaten zur Erfassung der physiologischen postnatalen Besiedlung und deren Stabilität. (2) Berechnung der Prävalenz potentiell pathogener Keime über den Beobachtungszeitraum.

Methoden. Analyse wöchentlicher mikrobiologischer Screeningabstriche aller Patienten der neonatologischen Intensivbehandlungsstation (BHS) und -überwachungsstation (ÜWS) der Abteilung, die im Zeitraum von Juli 2012 bis Juni 2014 geboren wurden. Zur Berechnung der longitudinalen Besiedlungskinetik wurden nur im Zentrum geborene Kinder berücksichtigt und der Prozentsatz an besiedelten Kindern über die Lebenszeit in Wochen berechnet. Die Prävalenz potentiell pathogener Keime ohne und mit erhöhter Antibiotikaresistenz wurde tagesgenau als Prozentsatz positiver Patienten pro Anzahl aller Patienten auf Station berechnet und dann wochenweise zusammengefasst.

Ergebnisse. Im erfassten Zeitraum wurden 3540 Rachen- und 2847 Analabstriche von insgesamt 1146 Patienten untersucht. Insgesamt wurden 50 verschiedene Erreger im Rachen und 55 in den Analabstrichen auf Speziesebene identifiziert. Während der ersten drei Lebenswochen (LW) waren Koagulase-negative Staphylokokken (KNS) die im Rachen am häufigsten nachgewiesenen Keime. Die Kolonisation mit vergrünenden Streptokokken erfolgte langsamer, ab der 4. Lebenswoche waren dies jedoch die meistisolierten Erreger. Im Analbereich waren Enterokokken und KNS in je ca. 40\% der Abstriche vorhanden. Die Besiedlung mit Enterokokken nahm jedoch rasch zu, so dass nach 5 Wochen bei 90\% der Patienten diese Keime nachweisbar waren, während die Kolonisation mit KNS über die Zeit zurückging. Die Besiedlung mit Enterobacteriaceae erfolgte langsam von $20 \%$ in LW 1 bis $80 \%$ in LW 12 zunehmend. Die Prävalenz potentiell pathogener Keime zeigte starke kurz- und längerdauernde Schwankungen. Enterobacteriaceae waren auf der ÜWS häufiger nachweisbar, während Staphylococcus (S.) aureus und S. haemolyticus auf der IBS eine höhere Prävalenz hatten. Escherichia coli wurde im Durchschnitt bei 36,2 (7,0-76,0)\% der ÜWSPatienten nachgewiesen, bei IBS-Patienten bei $15,2(0-51,7) \%$. Insgesamt wurden 57 (5,0\%) Patienten mit 2MRGN und 8 (0,07\%) Patienten mit 3MRGN erfasst. Die vorgeschriebenen Isolationsmaßnahmen führten zu 104 (1,8\%) gesperrten Bettentagen auf der IBS und 332 (6\%) auf der ÜWS (nur 2014).

Diskussion. Die Analyse der wöchentlich vorgeschriebenen Screeningabstriche gibt einen Einblick in die physiologische Rachen- und Rektal-Besiedlung. Während bis ca. 4 Wochen nach Geburt KNS in beiden Bereichen dominiert, bildet sich im Weiteren eine standortdifferenzierte Keimflora aus. Die Häufigkeit des Nachweises potentiell pathogener Keime ist stark schwankend, so dass die Erstellung von Normwerten schwierig erscheint. Der Nachweis von MRGN ist selten, kann aber zu erheblichen Beeinträchtigungen in der Zahl belegbarer Betten führen.

\section{Neonatologie: Sauerstofftherapie}

\section{FV49}

Fully automated predictive intelligent control of oxygenation (PRICO) in resuscitation and ventilation of preterm lambs

M. Hütten', T. Goos' ${ }^{2}$ D. Ophelders ${ }^{3}$, M. Nikiforou' ${ }^{3}$, E. Kuypers ${ }^{3}$, M. Willems ${ }^{3}$, H. Niemarkt ${ }^{4}$, J. Dankelman ${ }^{5}$, T. Mohns ${ }^{6}$, P. Andriessen ${ }^{6}$, T. Orlikowsky', I. Reiss ${ }^{8}$, B. Kramert

${ }^{1}$ Neonatologie, Klinik für Kinder- und Jugendmedizin, Universitätsklinikum, RWTH Aachen, and Department of Pediatrics, Maastricht University Medical Centre, Maastricht, The Netherlands, Aachen, Deutschland, ${ }^{2}$ Department of Pediatrics, Division of Neonatology, Erasmus Medical Centre, Rotterdam, and Department of Biomechanical Engineering, Delft University of Technology, Delft, NL, Rotterdam, Niederlande, ${ }^{3}$ Department of Pediatrics, Maastricht University Medical Centre, Faculty of Health, Medicine and Life Sciences, Maastricht, The Netherlands, Maastricht, Niederlande, ${ }^{4}$ Maastricht University Medical Center, Department of Pediatrics, Maastricht, Niederlande, ${ }^{5}$ Department of Biomechanical Engineering, Delft University of Technology, Delft, NL, Delft, Niederlande, ${ }^{6}$ Máxima Medical Center, Veldhoven, Neonatology, Veldhoven, Niederlande, ${ }^{7}$ Universitätsklinik für Kinder- und Jugendmedizin, Sektion für Neonatologie, Aachen, Deutschland, ${ }^{8}$ Erasmus MC-Sophia Children's Hospital, Dept. of Pediatric Surgery - Intensive Care, Rotterdam, Niederlande

Background. Hyperoxia and hypoxia influence morbidity and mortality of preterm infants. Therefore oxygen supplementation during resuscitation and ventilation of preterm infants has to be controlled within narrow saturation $\left(\mathrm{SpO}_{2}\right)$ targets. Automated closed-loop control of the fraction of inspired oxygen $\left(\mathrm{FiO}_{2}\right)$ has been shown to facilitate this difficult task in the NICU.

Hypothesis. We hypothesized that fully automated $\mathrm{FiO}_{2}$ control based on predefined $\mathrm{SpO}_{2}$ targets is applicable in both resuscitation and ventilation of preterm infants.

Study design. Twenty-two preterm lambs were operatively delivered and intubated in a modified EXIT procedure. They were randomized to receive standardized resuscitation with either automated or manual $\mathrm{FiO}_{2}$ control, targeting $\mathrm{SpO}_{2}$ according to the Dawson curve in the first 10 minutes and $\mathrm{SpO}_{2}$ 90-95\% hereafter. Starting $\mathrm{FiO}_{2}$ was either 0.3 or o.6. Automated $\mathrm{FiO}_{2}$ control was applied during surfactant replacement therapy and subsequent ventilation for 3 hours.

Results. Time within target range did not differ significantly between manual and automated $\mathrm{FiO}_{2}$ control during resuscitation, however automated $\mathrm{FiO}_{2}$ control significantly avoided hyperoxia (Abb. FV49.1). Automated $\mathrm{FiO}_{2}$ control was feasible during surfactant replacement and kept $\mathrm{SpO}_{2}$ within target range significantly better than manual control during subsequent ventilation (93.2\% [80.6-98.9\%] vs. $84.0 \%$ [63.8-89.4\%], p<0.05).

Comment. In our model, fully automated $\mathrm{FiO}_{2}$ control was applicable in rapidly changing physiologic conditions during postnatal resuscitation and surfactant replacement therapy and in stable conditions during subsequent ventilation. Although oxygenation in the first minutes of
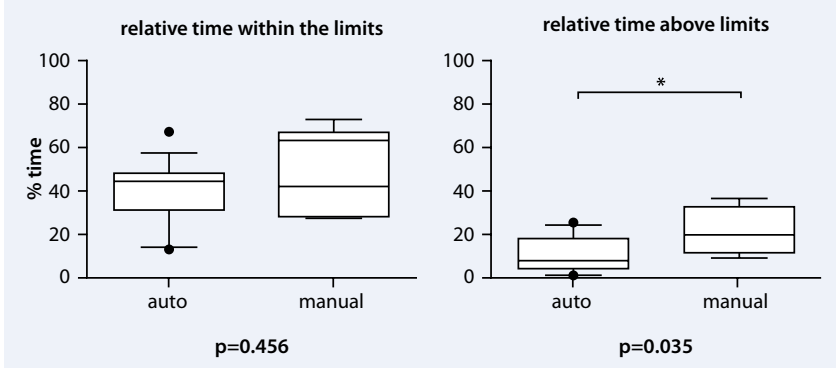

Abb. FV49.1 
life is influenced by a multitude of parameters which need to be managed by experienced personnel, automated $\mathrm{FiO}_{2}$ control might support preterm resuscitation.

\section{Lunchsymposium V: Tropenmedizin LAERDAL/CHIESI}

\section{FV50 \\ Ärzte ohne Grenzen - wer wir sind, was wir machen \\ N.N. ${ }^{?}$ \\ 'Ärzte ohne Grenzen e.V., Berlin}

„Ärzte ohne Grenzen“ ist eine private, internationale, medizinische Hilfsorganisation. Die Organisation hilft Menschen, die durch (Bürger-)Kriege oder Naturkatastrophen in Not geraten. „Ärzte ohne Grenzen" gewährt diese Hilfe allen Opfern, ungeachtet ihrer ethnischen Herkunft, politischen oder religiösen Überzeugung. Im Namen der universellen medizinischen Ethik und des Rechts auf humanitäre Hilfe arbeitet „Ärzte ohne Grenzen“ neutral und unparteiisch und fordert ungehinderte Freiheit bei der Ausübung ihrer Tätigkeit. Darüber hinaus engagiert sich die Organisation als Sprachrohr für Menschen in Not. Die Mitarbeiter beziehen öffentlich Stellung, wenn sie selbst Zeugen von massiven Menschenrechtsverletzungen oder schweren Verstößen gegen das humanitäre Völkerrecht werden. Jährlich arbeiten etwa 2000 internationale und rund 20.500 nationale Mitarbeiter von „Ärzte ohne Grenzen“ in mehr als 65 Ländern. Ihre Kompetenz und ihre Einsatzbereitschaft für Menschen in Not wurden 1999 mit dem Friedensnobelpreis geehrt.

Die Aktivitäten sind dabei sehr vielfältig: Wiederaufbau und Inbetriebnahme von Krankenhäusern oder Gesundheitszentren, mobile Kliniken zur Versorgung von ländlichen Gebieten, Impfprogramme, medizinische Versorgung in Flüchtlingslagern, psychologische Betreuung, Aufbau von Ernährungszentren, Wasser- und Sanitärprojekte sowie Gesundheitsversorgung von besonders gefährdeten Gruppen (z. B. Straßenkinder, Slumbewohner). Wir arbeiten in allen Projekten mit nationalem Personal zusammen und legen Wert auf die Fort- und Weiterbildung der einheimischen Mitarbeiter.

Ansprechpartner: Ärzte ohne Grenzen, Am Köllnischen Park 1, 10179 Berlin, Tel.: 030-700 130 o, E-Mail: office@berlin.msf.org, www.aerzteohne-grenzen.de

\section{Poster}

\section{Posterwalk 1 - Freie Themen 1}

\section{P01-01 \\ Schwere obere gastrointestinale Blutungen durch Ulzera bei gesunden Reifgeborenen}

\section{Krüger', S. Schweizer', G. Damm}

'St. Franziskus Hospital, Klinik für Kinder- und Jugendliche, Ahlen, Deutschland

Einführung. Gastrointestinale (GI) Blutungen in den ersten Lebenstagen sind eher selten, am ehesten treten sie bei schwer kranken Früh- und Neugeborenen auf. Wir berichten über 2 gesunde, männliche Neugeborene mit ausgeprägten Blutungen im oberen Gastrointestinaltrakt am ersten Lebenstag.

Kasuistiken. Die Neugeborenen wurden in der 40. bzw. 41. SSW (GG $4220 \mathrm{~g} / 3150 \mathrm{~g})$ spontan bzw. per Sektio nach normalem Geburtsverlauf (jeweils APGAR 9/10/10; NS-pH 7,35/7,37) geboren. Sie erhielten Vitamin K oral und wurden nach Geburt gestillt. Die Mutter des ers- ten Kindes war gesund und hatte in der Schwangerschaft nur Antazida wegen Sodbrennen erhalten. Die Mutter des zweiten Kindes hatte einen Gestationsdiabetes und eine behandelte Hypothyreose. 18 Stunden nach Geburt erbrach Kind 1 massiv frisches Blut und setzte wenig später frisch-blutigen Stuhl ab. Der Blutverlust erforderte eine Transfusion. Kind 2 erbrach 12 Stunden nach Geburt viel frisches Blut, der Stuhl war makroskopisch normal (okkultes Blut positiv). Die Hämoglobinkonzentration blieb stabil. Eine Sepsis oder Gerinnungsstörung lag bei beiden nicht vor. Bei V. a. eine obere GI-Blutung erhielten die Kinder Pantoprazol o,5 mg/kg i.v. 2-mal täglich. Bei der Gastroskopie am 2. Behandlungstag waren keine aktiven Blutungen mehr zu sehen, jedoch Ulzera im distalen Ösophagus und Fundusbereich (Kind 1) bzw. eine schwere ulzerierende Ösophagitis im distalen Ösophagus und eine ausgeprägte Gastritis (Kind 2) als wahrscheinliche Blutungsquellen. Im Stuhl konnte Helicobacter-pylori(H.p.)-Antigen nur bei Kind 1 gefunden werden, nicht aber in dessen histologischen Proben (PCR ebenso negativ). Bei der Mutter dieses Kindes wurde eine H.p.-assoziierte Gastritis diagnostiziert. Die Pantoprazol-Therapie bei Kind 1 wurde über insgesamt 4 Wochen durchgeführt, eine Kontrollendoskopie zeigte eine völlige Abheilung. Bei Kind 2 wurde Pantoprazol über 8 Wochen gegeben, da eine Kontrollendoskopie nach 5 Wochen noch Reste der Gastritis zeigte. Die weitere Entwicklung der Kinder war normal.

Schlussfolgerung. Auch bei gesunden Neugeborenen kann es in den ersten Lebenstagen zu schweren GI-Blutungen kommen, ohne dass offensichtliche maternale oder neonatale Risikofaktoren vorliegen. Bei unseren Patienten lag an in der Literatur beschriebenen Risikofaktoren bei Kind 1 nur eine Antazida-Einnahme in der Schwangerschaft, bei Kind 2 eine Kaiserschnittentbindung vor. Die H.p.-Besiedlung bei Kind 1 muss als nicht pathogen gewertet werden. Beide wurden gestillt, was als protektiver Faktor angesehen wird. Eine Endoskopie sollte stets angestrebt werden. Die Blutungen werden, wie hier berichtet, in der Regel durch Ulzera in Ösophagus und Magen verursacht. Eine Protonenpumpenhemmer-Therapie ist regelhaft indiziert, weitere supportive Maßnahmen (z. B. Transfusion) können notwendig werden. Die Prognose ist exzellent. Fall-Kontroll-Studien in einer großen Population, z. B. über eine ESPED-Erhebung, werden notwendig sein, um die Ursachen besser verstehen zu lernen.

\section{P01-02 \\ Leistenhernien bei Frühgeborenen - gibt es einen optimalen Zeitpunkt für die Operation?}

P. Franck', J. Rädecke ${ }^{2}$, R. Hentschel'

'Universitätsklinikum, Zentrum für Kinder- und Jugendmedizin, Neonatologie/Intensivmedizin, Freiburg, Deutschland, ${ }^{2}$ Universitätsklinikum, Chirurgische Klinik, Kinderchirurgie, Freiburg, Deutschland

Hintergrund. Der beste Zeitpunkt für die elektive OP einer Leistenhernie (LH) bei Frühgeborenen (FG) ist umstritten. Der Sorge vor einer pulmonalen Verschlechterung, einem schlechteren OP-Ergebnis und einem höheren Narkoserisiko bei einer zu frühen OP steht das Risiko einer Inkarzeration durch zu langes Warten gegenüber.

Methode. Retrospektive Datenerfassung aus dem Krankenhausinformationssystem über 10 Jahre (2004-2013): Alle stationären Fälle im ersten Lebensjahr mit den ICD-Codes Po7.3 (FG) und K40. (LH), die am Universitätsklinikum Freiburg operiert wurden. Analyse von OPZeitpunkt im ersten oder in einem Folgeaufenthalt, perioperativer Verlegung auf die Intensivstation, Beatmung, Beatmungsintensität, Inkarzeration, Inkarzerationsfolgen $u$. a.

Ergebnisse. Patientenkollektiv: 106 konsekutive LH-OP-FG mit einem mittleren GA von 28 SSW (22-36), GG von $1060 \mathrm{~g}$ (430-2150), 88\% männlich. Anteil BPD 30\%, komplizierende chirurgische oder neurologische Diagnosen $28 \%$. OP-Zeitpunkt: $35 \%$ im ersten, $65 \%$ in einem Folgeaufenthalt. Mittleres Alter bei OP 3,8 Monate (0,5-20), mittleres Gewicht $3200 \mathrm{~g}$ (1340-7900). Perioperativ auf Intensivstation (IS): $28 \mathrm{~Pa}$ tienten lagen post-op. auf der IS, davon waren 21 beatmet. Indikationen für IS: OP von der IS aus $(\mathrm{N}=17$, davon $3 \mathrm{LH}-\mathrm{OPs}$ als Teil komplexerer 
OPs), elektive Narkoseausleitung ( $\mathrm{N}=11$, davon 7 nach Inkarzeration). Nur in einem Fall Verlegung auf IS bei elektiver OP im ersten Aufenthalt (CPAP und HFNC für 24 Stunden). Inkarzerierte LH (ink.LH): 23 (22\%), davon 16 (70\%) während des ersten Aufenthalts. OP-Zeitpunkt bei ink. LH zu 61\% (14/23) außerhalb der Regeldienstzeit (bei den von zuhause kommenden Fällen zu 86\%). 5 der ink. LH ließen sich nach Narkosebeginn reponieren. Folgen der ink. LH: 78\% Ödem, 16\% livider Hoden, 13\% Anzeichen von Darmperfusionsstörung, 15\% (Sub-)Ileus. Kein Fall einer Darmresektion. Spätere OP auf der Gegenseite: 2 Rezidive, 9 spätere OPs auf der Gegenseite. Zweit-OP auf der Gegenseite v.a. nach Primär-OP einer ink. LH (ink. LH bei Kindern mit Zweit-OP $89 \%$, vs. $15 \%$ im Kollektiv ohne Zweit-OP) und bei jungem OP-Alter $(1,4$ Monate, vs. 4,o Monate in der Gruppe ohne Zweit-OP).

Schlussfolgerung. Die elektive LH-OP bei anderweitig stabilen FG am Ende des ersten Klinikaufenthalts scheint ohne zusätzliche Risiken durchführbar. Die Mehrzahl der Inkarzerationen trat unter stationärer Überwachung während des Primäraufenthalts auf. Alle Fälle von Inkarzeration blieben ohne schwerere Organfolgen. Bei früher elektiver oder ink. LH-OP ist das Risiko einer späteren Zweit-OP auf der Gegenseite zu beachten. Eine individuelle Entscheidung nach Abwägung der Risiken, u.a. auch unter Berücksichtigung der Wohnortnähe zur Klinik, ist nötig.

\section{P01-03}

\section{Der Einfluss des Gestationsalters auf das Outcome von Kindern} mit Gastroschisis

\section{Aydin', S. Filippidou'², D. Katzer ${ }^{3}$, A. Heydweiller', A. Mueller , S. Bagci}

'Zentrum für Kinderheilkunde, Universität Bonn, Bonn, Deutschland, ${ }^{2}$ Zentrums für Geburtshilfe und Frauenheilkunde, Bonn, Deutschland, ${ }^{3}$ Zentrum für Kinderheilkunde, Universität Bonn, Neonatologie und Pädiatrische Intensivmedizin, Bonn, Deutschland, ${ }^{4}$ Chirurgische Klinik, Universität Bonn, Kinderchirurgie, Bonn, Deutschland

Hintergrund. Der Einfluss des Gestationsalters auf das Outcome sowie der optimale Entbindungszeitpunkt bei Kindern mit Gastroschisis (GS) werden zur Zeit kontrovers diskutiert. Die Hypothese ist, dass durch die Langzeitexposition der frei flottierenden Darmschlingen im Fruchtwasser in der Spätschwangerschaft mit zunehmender Schwangerschaftsdauer ein erhöhtes Risiko für entzündliche Darmwandschädigungen besteht. Eine vorzeitige Entbindung vor der 36. SSW könnte diese Gefahren verhindern.

Fragestellung. Einfluss des Gestationsalter auf das Outcome von Kindern mit Gastroschisis (GS).

Methodik. Retrospektive Analyse von 52 Kindern mit GS. Als Outcome-Parameter wurden der Tag des ersten Stuhlgangs (eSG), der Beginn des oralen Nahrungsaufbaus mit Milch (bNA), die Dauer des enteralen Nahrungaufbaus [mehr als $100 \mathrm{ml} / \mathrm{kg} / \mathrm{d}$ (NA10o) und die Vervollständigung des oralen Nahrungsaufbaues (vNA)], die Dauer des Krankenhausaufenthalts (KA), das Gestationsalter bei Entlassung (eGA), sowie die postoperativen Komplikationen während des stationären Aufenthaltes analysiert.

Ergebnisse. Das mediane Gestationsalter (GA) (min.-max.) betrug 35,7 SSW $(32,6-38,1)$. Statistisch zeigte sich eine signifikante negative Korrelation zwischen dem Gestationsalter (GA) und den Parametern KA $(p<0,001), e G A(p=0,016), N A 100(p=0,008)$, sowie vNA $(p<0,001)$. Zwischen dem GA und den Parametern eSG $(p=0,218)$ und bNA $(p=0,878)$ konnte hingegen keine signifikante Korrelation gezeigt werden. Das Auftreten einer postoperativen Sepsis korrelierte invers mit dem GA ( $\mathrm{p}=\mathrm{o}, \mathrm{o19}$ Im Vergleich mit den Kindern ohne Komplikation und/oder Sepsis waren GA $(p=0,049), K A(p<0,001), e G A(p<0,001)$, NA10o $(\mathrm{p}=\mathrm{o}, \mathrm{oo2})$, sowie vNA ( $\mathrm{p}<0,001)$ bei Kindern mit Komplikation und/ oder Sepsis statistisch signifikant länger/höher.

Schlussfolgerung. Unsere Ergebnisse zeigen, dass ein früherer Entbindungszeitpunkt von Kindern mit GS mit einem längeren Krankenhausaufenthalt, protrahierten Nahrungsaufbau und einer höheren Sepsisrate verbunden ist. Der in der Literatur häufig empfohlene frühere
Entbindungstermin bei Kindern mit GS kann durch unsere Daten nicht unterstützt und sollte überdacht werden.

\section{P01-04}

Neonatale Nierenvenenthrombose bei diabetischer Fetopathie, Vitamin-B12-Mangel und MTHFR-Mutation

\section{Henn', D. Wolf', H. Schneider', P. Freisinger ${ }^{2}$}

'Klinikum am Steinenberg Reutlingen, Klinik für Kinder- und Jugendmedizin, Reutlingen, Deutschland, ${ }^{2}$ Kreiskliniken Reutlingen, Klinik für Kinderund Jugendmedizin, Reutlingen, Deutschland

Einleitung. In der Neonatalperiode ist die Nierenvenenthrombose die zweithäufigste thrombembolische Erkrankung. Insgesamt wird von einer Inzidenz von 2,2/1000oo Lebendgeburten ausgegangen. Als wichtigster Risikofaktor wird mit 53\% das Vorliegen eines prothrombotischen Faktors (z. B. Protein C- und S-Mangel, Faktor-V-Leiden und Prothrombinmutationen) beschrieben. Agravierend hierfür sind eine peripartale Asphyxie, maternaler Diabetes mellitus, Sepsis und Nabelvenenkatheter. Wir berichten über ein Neugeborenes mit Nierenvenenthrombose, diabetischer Fetopathie und MTHFR-Mutation.

Fallbeschreibung. Das Kind wurde in der 40. Schwangerschaftwoche als zweites Kind einer 25-Jährigen spontan nach Einleitung bei silentem CTG und Polyhydramnion geboren. NapH 7,28, Apgar 4/7/9, GG 4430 g (100 g >97 P). Postpartal erfolgte eine CPAP-Beatmung. Bei $\mathrm{CO}_{2}$-Retention, metabolisch und respiratorischer Azidose Intubation und Surfactantgabe (FiO2 max. 100\%). Initial bestanden bei klinischem Bild einer diabetischer Fetopathie auch Hypoglykämien (min. $22 \mathrm{mg}$ / dl). Laborchemisch zeigten sich stark erhöhte D-Dimere (>5000 ng/ $\mathrm{ml}$ ), eine LDH von $663 \mathrm{U} / \mathrm{l}$ sowie eine Makrohämaturie. Sonographisch Nierenvenenthrombose rechts mit vergrößerter inhomogener Niere, erhöhter Echogenität und verminderte kortikomedulläre Differenzierung. Im Thrombophilie Screening zeigte sich eine heterozygote MTHFR-Mutation $\left.\left(\mathrm{C}_{67} \mathrm{~T}\right) \mathrm{T}\right)$ mit Homocysteinämie $(21,5 \mu \mathrm{mol} / \mathrm{l}$ - NB <12,4 $\mu \mathrm{mol} / \mathrm{l}$ ) mit einem Vitamin-B12-Spiegel von $88 \mathrm{pg} / \mathrm{ml}(\mathrm{NB}$ 190-980 pg/ml) bei manifestem alimentärem Vitamin-B12-Mangel der Mutter. Der Vitamin-B12-Mangel führt zusätzlich zu einer Erhöhung von Homocystein. Behandelt wurde leitliniengerecht mit niedermolekularem Heparin (NMH - Clexane $2 \times 1,5 \mathrm{mg} / \mathrm{kg} / \mathrm{d})$, unter anti FaktorXa Monitoring. Eine antithrombotische Sekundärprophylaxe erfolgte im Anschluss. Im Alter von 6 Monaten fand sich eine Schrumpfniere rechts, linke Niere kompensatorisch vergrößert. Auf die antithrombotische Prophylaxe konnte, bei geringer Wahrscheinlichkeit für Appositionsthromben, verzichtet werden. Bei MTHFR- Mangel empfahlen wir auch im Kindesalter die Gabe von NMH bei Immobilisation. Auch sollte im weiteren Verlauf die Entwicklung einer arteriellen Hypertonie beachtet werden.

Schlussfolgerung. Bei unserer Patientin zeigt sich eine Nierenvenenthrombose bei heterozygoter MTHFR-Punktmutation, diabetischer Fetopathie sowie einem Vitamin-B12-Mangel. Man findet eine MTHFR-Mutation bei ca. $10 \%$ der Normalpopulation. Bei Koinzidenz mit weiteren prothrombotischen Faktoren, insbesondere bei präexistentem Vitamin-B12-Mangel potenziert sich das Risiko für neonatale thrombembolische Ereignisse. Weiter zeigten Koch et al ein signifikant erhöhtes Thromboserisiko bei Vorliegen einer CT-Variante der Mutation (hier C677T-Mutation). 


\section{P01-05}

\section{Cellular and humoral coagulation profiles and occurrence of IVH in VLBW and ELWB infants}

\section{P. Duppré', L. Gortner', S. Wagenpfeiß', J. Geisel', S. Gottschling', S. Meyer' ${ }^{1}$ Universitätsklinikum des Saarlandes, Klinik für Allgemeine Pädiatrie und Neonatologie, Homburg, Deutschland, ${ }^{2}$ Unversitätsklinik für Kinder und Jugendmedizin Gebäude ${ }^{9}$, Homburg, Deutschland, ${ }^{3}$ Institut für Epidemio- logie, Biometrie und Medizinische Informatik, Homburg, Deutschland, ${ }^{4}$ Universitätsklinikum des Saarlandes, Zentrallabor, Homburg, Deutschland, ${ }^{5}$ Universitätsklinikum des Saarlandes, Klinik für Kinder- und Jugendmedi- zin, Zentrum für Kinderschmerztherapie und Palliativmedizin, Homburg, Deutschland}

Background. Intraventricular hemorrhage (IVH) is a major complication in preterm neonates with significant long-term morbidity and an increased mortality rate. The role of the immature coagulation system in the pathogenesis in these infants is still under debate. The aim of this study was to provide reference values for coagulation studies within the first 24 hours of life, and to relate these findings to the incidence of IVH of any grade.

Patients and methods. In this retrospective study, a total of 250 (male: 123/female: 127) VLBW (150) and ELBW (100) infants were included over a 4-year-period. Coagulation studies were performed within the first $24 \mathrm{~h}$ of life in all infants. Multiple regression analysis was employed to demonstrate a potential association between IVH and a number of known risk and protective factors for IVH (antenatal steroids, birth weight/gestational age, gender, IUGR, APGAR score at 10 minutes, platelet count, INR, PTT and fibrinogen).

Results. Mean birth weight was 1047,9 $\pm 305,6$ (range: 320-1490 g). Both cellular (platelets, nucleated red blood cells) and plasmatic coagulation parameters (INR, fibrinogen and antithrombin III) were dependent on birth weight. Moreover, INR levels $(\mathrm{p}<0,05)$ were significantly lower in neonates with IVH of any grade. Also, INR was positively correlated with the severity of IVH (Spearman's correlation coefficient: 0,193; $\mathrm{p}=\mathrm{o}, \mathrm{oO} 3$ ).

Conclusions. Our data provide a robust set of reference values for both cellular and humoral coagulation studies in VLBW and ELBW infants for the first 24 hours of life. The results of our study indicate that abnormal INR levels are significantly associated with the occurrence of IVH of any grade in this susceptible cohort.

\section{P01-06 \\ Hämolytische Anämie beim Neugeborenen: verlässliche Diagnosestellung einer hereditären Sphärozytose durch Kombi- nation moderner Analysemethoden}

\section{O. Andres', S. Eber', C. Speer}

'Universitäts-Kinderklinik Würzburg, Neonatologie und Pädiatrische Intensivmedizin, Würzburg, Deutschland, ${ }^{2} S$ chwerpunktpraxis für Neonatologie und Pädiatrische Hämatologie, München, Deutschland, ${ }^{3}$ Universitäts-Kinderklinik Würzburg, Würzburg, Deutschland

Hintergrund. Die hereditäre Sphärozytose ist die häufigste angeborene chronische hämolytische Anämie in Mitteleuropa. Wegen defekter struktureller Membranproteine ist sie durch eine erhöhte osmotische Fragilität und einen erhöhten Membranverlust der Erythrozyten gekennzeichnet. Da der Nachweis von Sphärozyten im Blutausstrich eines Neugeborenen physiologisch ist und neonatale Erythrozyten generell eine leicht erhöhte osmotische Resistenz aufweisen, wird die Diagnosestellung einer hereditären Sphärozytose direkt postnatal als nicht verlässlich, häufig sogar als nicht möglich angesehen. Eine frühe postnatale Diagnosestellung kann jedoch zur Identifizierung der Patienten beitragen, die durch eine verstärkte oder verspätete indirekte Hyperbilirubinämie, einen Kernikterus oder eine rasch einsetzende Transfusionsbedürftigkeit besonders gefährdet sind.
Fragestellung. Lässt sich die hereditäre Sphärozytose mit modernen Analysemethoden im Neugeborenenalter verlässlich stellen? Patienten und Methoden. In unserem spezialisierten Erythrozytenlabor haben wir seit 2010 mehr als 500 Blutproben zur Abklärung einer hereditären Sphärozytose mittels morphologischer Beurteilung des Blutausstrichs, dem photometrischen Acidified Glycerol Lysis Test (AGLT) und dem durchflusszytometrischen Eosin-5'-Maleimid-Test (EMA) analysiert. Für 37 Einsendungen von Neugeborenen und jungen Säuglingen bis zum Alter von sechs Wochen haben wir das durchflusszytometrische Verfahren an das erhöhte mittlere korpuskuläre Volumen (MCV) neonataler Erythrozyten angepasst. Von elf Patienten erhielten wir Blutproben aus der ersten Lebenswoche, von einer Patientin erst nach erfolgter Erythrozytentransfusion. Bei 14 Patienten war ein Elternteil an der meist autosomal-dominant vererbten Erythrozytenanomalie erkrankt.

Ergebnisse. Durch Kombination der beiden modernen Messmethoden AGLT und EMA sowie der Beurteilung der Erythrozytenmorphologie gelang es, die Verdachtsdiagnose einer hereditären Sphärozytose bei 12 Patienten, darunter der Patientin nach Transfusion, eindeutig zu sichern und bei 21 Patienten klar auszuschließen. Bei zwei Patienten konnten andere seltene Diagnosen morphologisch gestellt werden. Bei vier weiteren Patienten mit inkongruenten Testergebnissen nach verlängertem Probentransport ergab die empfohlene spätere Wiederholungsuntersuchung einen Ausschluss der Erkrankung oder den Nachweis einer milden Form. Durch Vergleich der automatisiert erhobenen Erythrozytenindizes konnte der Anteil der hyperdensen Zellen als hochsignifikanter Parameter zur Mitbeurteilung einer hereditären Sphärozytose ermittelt werden.

Schlussfolgerung. Die Diagnose einer hereditären Sphärozytose kann im Neugeborenenalter durch Kombination der Analyseverfahren AGLT und EMA auch aus kleinen Blutmengen (0,5 ml EDTA-Blut) und nach raschem Versand verlässlich gestellt werden. Unsere Daten belegen, dass eine Blutprobe schon bei Verdacht auf eine erythrozytäre Erkrankung zum frühestmöglichen Zeitpunkt entnommen und einer spezialisierten Erythrozytendiagnostik zugeführt werden sollte.

\section{P01-07 \\ Kurzfristige Auswirkungen von Bluttransfusionen auf die Hepcidin-Konzentration bei Frühgeborenen}

K. Müller', L. Lorenz', C. Poets' ${ }^{1}$ A. Peter', G. Olbina', M. Westerman ${ }^{4}$, A. Franz ${ }^{1}$

'Eberhard-Karls-Universität Universitätsklinik für Kinderheilkunde und Jugendmedizin, Neonatologie, Tübingen, Deutschland, ${ }^{2}$ Laboratoriumsmedizin, Klinische Chemie und Pathobiochemie, Medizinische Klinik, Universität Tübingen, Tübingen, Deutschland, ${ }^{3}$ nntrisic LifeSciences, La Jolla, USA, ${ }^{4}$ Intrinsic LifeSciences, Tübingen, Deutschland

Hintergrund. Hepcidin, ein Regulator der Eisenhomöostase, könnte in der Zukunft ein wichtiger nicht-invasiver Parameter zur Überwachung der Eisensupplementierung bei Frühgeborenen sein. Um Hepcidin richtig zu interpretieren, müssen Faktoren, die die Hepcidinkonzentrationen beeinflussen, in Betracht gezogen werden. Ziel war, zu evaluieren, ob die Transfusion von Erythrozytenkonzentrat (EK) einen nachweisbaren Effekt auf die Konzentrationen von Hepcidin im Serum $(\operatorname{Hep}(\mathrm{S}))$ und im Urin $(\mathrm{Hep}(\mathrm{U}))$ zeigt.

Methoden. Dies ist eine prospektive Beobachtungsstudie an Extremfrühgeborenen, die eine EK-Transfusion benötigten. Vor und nach EK-Transfusion wurde die Konzentration der 25-Aminosäuren langen Form von Hepcidin im Serum und Urin mit Hilfe eines ELISAs gemessen. Zeitgleich wurde auch ein Blutbild bestimmt.

Ergebnisse. Die Studie wurde von Mai 2009 bis September 2010 an der Universitätsklinik Tübingen durchgeführt. 20 Frühgeborenen mit einem mittleren Gestationsalter von 26+6/7 Schwangerschaftswochen (Interquartilsabstand $24+6 / 7$ bis $27+3 / 7$ ) erhielten insgesamt 27 EK-Transfusionen im korrigierten Alter von $31+3 / 7$ Schwangerschaftswochen [Interquartilsabstand 29+6/7-34+5/7)]. Nach Transfusion [im Mittel 10 Stunden (Standardabweichung (SD): 6] stiegen die Hämatokritwerte von 


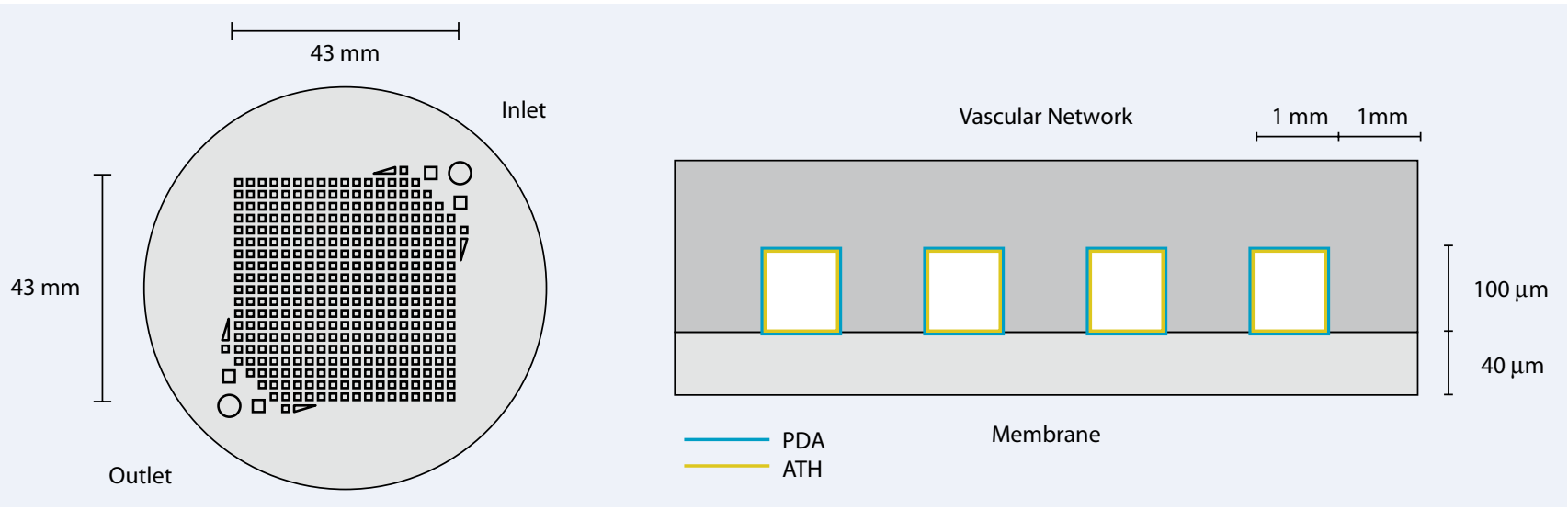

Abb. P01-08.1 Schematic of an SOU 4

im Mittel 26,6\% (SD 2,8) auf 40,9\% (SD 3,2; $\mathrm{p}<0,0001)$. Auch zeigte sich ein signifikanter Anstieg der Hep(S) [geometrisches Mittel: 44,3 ng/mL (95\% Konfidenzintervall: $30,8-63,8)$ vs. 58,0 ng/ml (95\% Konfidenzintervall: 35,7-94,3; $\mathrm{p}<0.05)$ ]. Im Urin konnte kein Anstieg der Hepcidinkonzentration nach Transfusion nachgewiesen werden.

Schlussfolgerung. Die Daten zeigen einen kurzfristigen Effekt von EKTransfusionen auf die Serum Hepcidinkonzentration bei Frühgeborenen. Um aber die Dynamik der Hepcidinregulation bei Frühgeborenen vollständig zu verstehen sind weitere Untersuchungen notwendig.

\section{P01-08}

Artificial placenta: surface modification of single oxygenator units (SOUs) using polydopamine (PDA) as bio-glue for anticoagulant antithrombin-heparin (ATH) complex

D. Sandejas', R. Cornelius', L. Berry', N. Rochow', H. Matharoo', G. Fusch', H. Atkinson ${ }^{6}$, A. Chan ${ }^{3}$, R. Selvaganapathy ${ }^{5}$, C. Fusch ${ }^{7}$, J. Brash ${ }^{8}$

${ }^{1}$ Biomedical Engineering, McMaster University, Hamilton, Hamilton, Kanada, ${ }^{2}$ Chemical Engineering, McMaster University, Hamilton, Kanada, ${ }^{3}$ Pediatrics, McMaster University and Thrombosis and Atherosclerosis Research Institute, Hamilton, Kanada, ${ }^{4}$ Pediatrics, McMaster University, Hamilton, Kanada, ${ }^{5}$ Mechanical Engineering, McMaster University, Hamilton, Kanada, ${ }^{6}$ Thrombosis and Atherosclerosis Research Institute, Hamilton, Kanada, ${ }^{7}$ Pediatrics, McMaster University and Biomedical Engineering, McMaster University, Hamilton, Kanada, ${ }^{8}$ Biomedical Engineering, McMaster University and Chemical Engineering, McMaster University, Hamilton, Kanada

Background. Our "artificial placenta" neonatal lung assist device is a stacked array of microfluidic polydimethylsiloxane (PDMS) SOUs (Abb. Po1.08.1; Schematic of a SOU). Blood contacting surfaces within the SOUs need to be hemocompatible, A method using PDA as a 'bioglue' to attach a covalent ATH complex, a potent anticoagulant, has been developed to achieve hemocompatibility.

Objective. To quantify ATH binding and bioactivity on PDA modified SOUs.

Methods. Quantification and evaluation of surface bound ATH was carried out through blood exposure. Two sets of oxygenators ( $\mathrm{n}=6$ total) were incubated in dopamine hydrochloride $(1 \mathrm{mg} / \mathrm{mL}$ in $\mathrm{PBS}, \mathrm{pH} 8.5$, $24 \mathrm{hrs}$ ) which oxidizes to PDA. Subsequent ATH incubation formed the PDMS-PDA-ATH complex (o.1 mg/mL in PBS, pH 7.4, $24 \mathrm{hrs).} 125 \mathrm{I}-\mathrm{la}-$ beled ATH was used as a tracer. One set of SOUs $(n=3)$ was then exposed whole blood (hematocrit 0.5) for 2 days. The heparin component of $\mathrm{ATH}$, if active, selectively binds antithrombin (AT); therefore, the anticoagulant activity of ATH modified SOUs was evaluated by measuring AT uptake from plasma ( $3 \mathrm{hrs}, \mathrm{n}=6)$ to PDMS-PDA-ATH. 125I-labeled AT was added to plasma as a tracer.

Results. Initially, $0.23 \mu \mathrm{g} / \mathrm{cm} 2$ of ATH was bound to the PDMS-PDA oxygenators after 24 hours. This level suggests monolayers were formed.

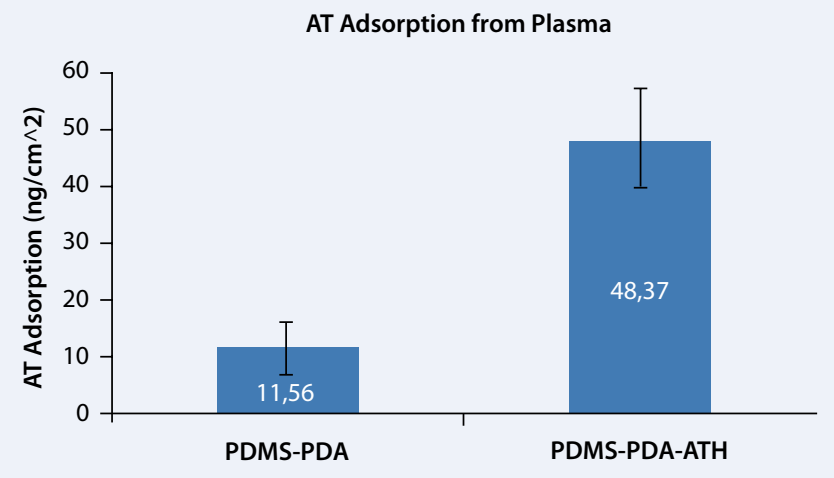

Abb. P01-08.2 Bioactivity of PDMS-PDA and PDMS-PDA-ATH surfaces $\triangle$

Subsequent exposure to blood removed $35 \%$ after 48 hours, with $0.15 \mu \mathrm{g} /$ $\mathrm{cm} 2$ of ATH remaining on the surface. This indicates the binding of ATH to PDMS-PDA was relatively stable. Abb. Po1-08.2 shows that PDMS-PDA-ATH SOUs bound $48.4 \mathrm{ng} / \mathrm{cm} 2$ of AT from plasma, significantly higher than the precursor PDMS-PDA, at $0.15 \mathrm{ng} / \mathrm{cm} 2$. This demonstrates that the anticoagulant activity of heparin in ATH remains active when attached through PDA.

Conclusions. PDA, used as an adhesive agent to attach ATH to PDMS microfluidic SOUs, provides high ATH surface density, increased stability, and increased anticoagulant activity.

\section{P01-09}

Artificial placenta: postnatal dilatation of umbilical cord vessels and its impact on wall integrity

J. Peng', N. Rochow', J. Jansen', B. DeFrance ${ }^{3}$, S. Lee', C. Fusch'

${ }^{1}$ Pediatrics, McMaster University, Hamilton, Kanada, ${ }^{2}$ Pathology and Molecular Medicine, McMaster University, Hamilton, Kanada, ${ }^{3}$ McMaster University, Obstetrics and Gynocology, Hamilton, Kanada

Introduction. In Neonatal Intensive Care Units, respiratory insufficiency among low-birth weight infants has been a major cause of mortality. For preterm infants, central umbilical cannulation has been widely performed for administering antibiotics, electrolytes, and nutrients. These umbilical vessels can become a source of vascular access to connect the preterm infant to an artificial placenta (AP) that provides sufficient gas exchange to overcome respiratory distress. The concept of an AP requires large bore access via umbilical vessels. In utero, umbilical artery (UA) and vein (UV) have wide diameters (3-5 and 6-10 $\mathrm{mm}$, respectively). After birth, these vessels constrict, and need to be re-expanded to maintain high extracor- 


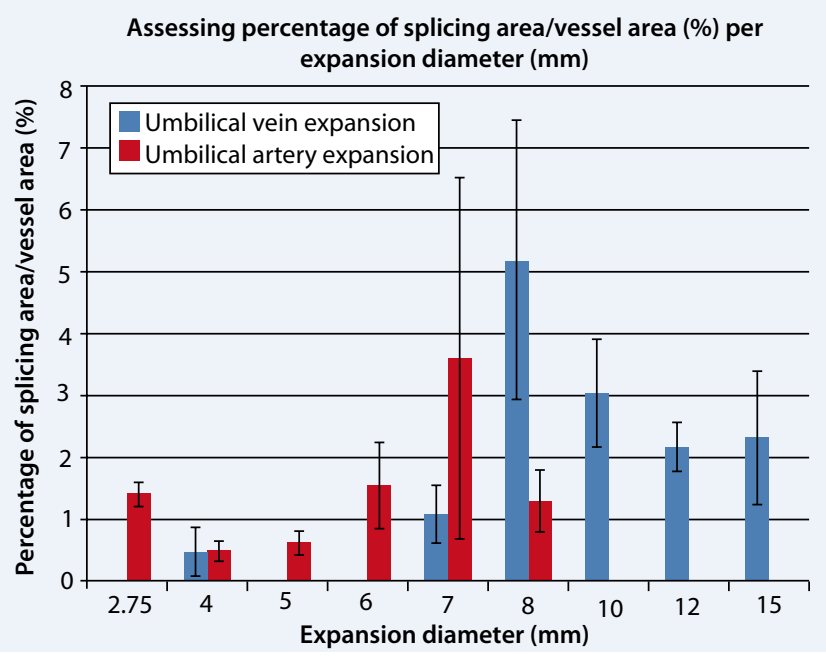

Abb. P01-09.1 4

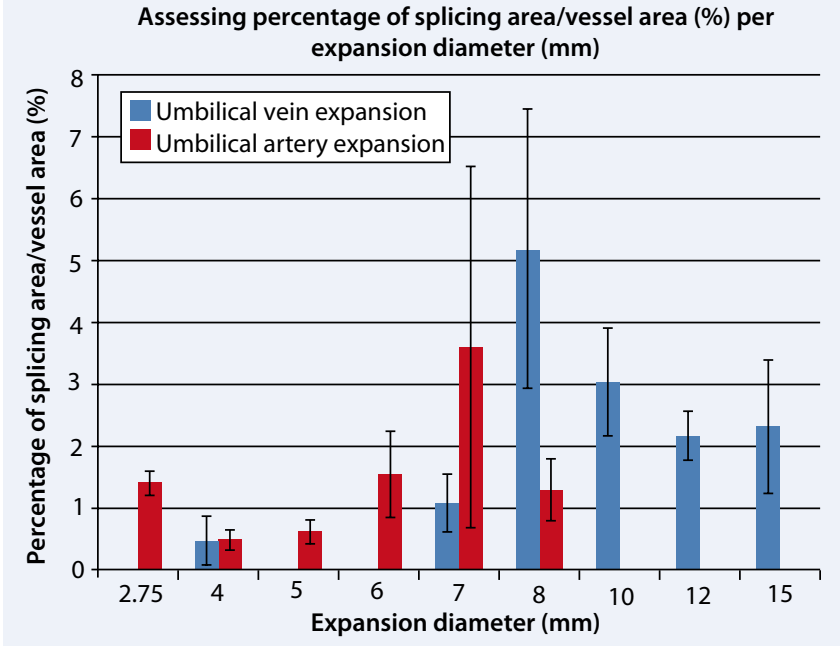

Abb. P01-09.3 $\triangle$
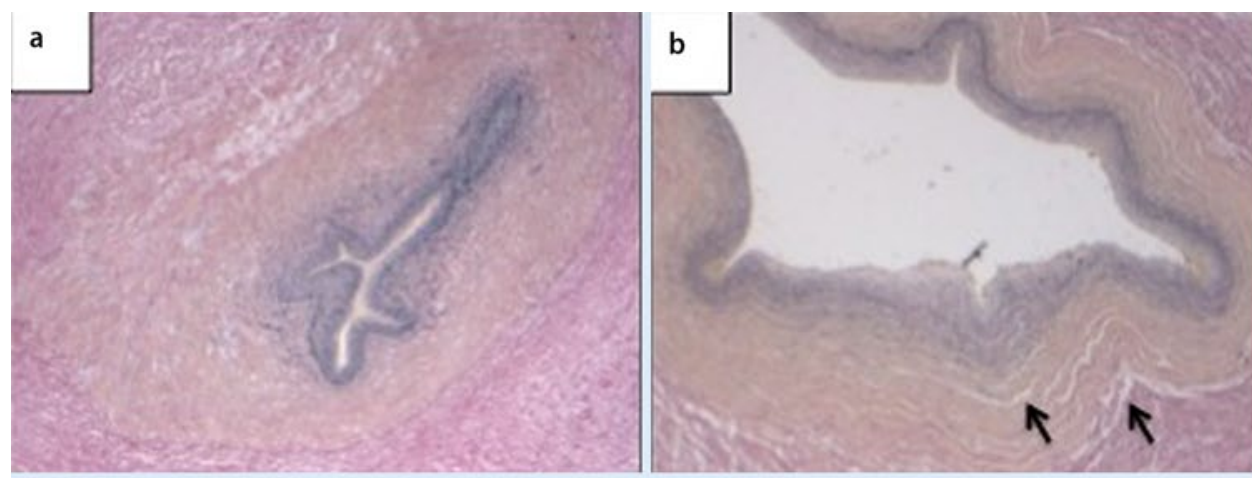

Umbilical artery dilatations

a) Control b) $5 \mathrm{~mm}$
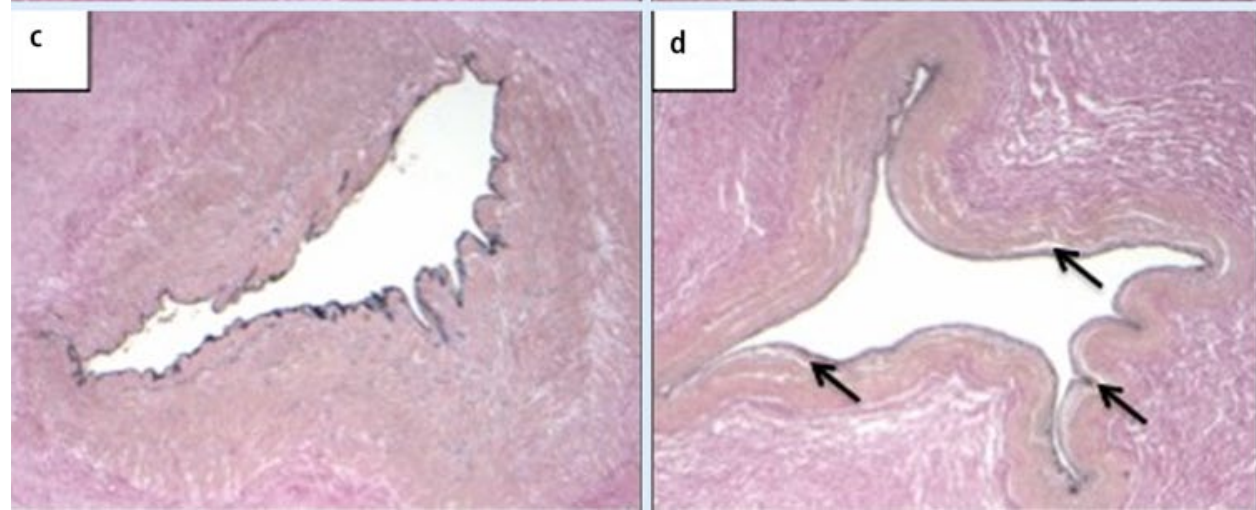

Umbilical vein dilatations

c) Control d) $12 \mathrm{~mm}$

Black arrows indicate splicing

Abb. P01-09.2 $\triangle$

poreal flow rate for successful application of an AP. However, re-opening of the UA and UV via catheterization may compromise vessel wall integrity. Objective. To study the impact of expansion on the vascular integrity of the UA and UV and identify a threshold diameter for safe expansion.

Methods. Umbilical cords were collected from term pregnancies $(\mathrm{N}=12)$. Cannulation and dilatation were performed in UA and UV from cord sections by inflating percutaneous transluminal angioplasty (PTA) catheters (3-8 $\mathrm{mm}$ for UA and 4-15 $\mathrm{mm}$ for UV). Upon $30 \mathrm{~s}$ dilatation, cord sections were harvested. Paraffin-embedded transverse sections ( $4 \mu \mathrm{m}$ thickness) were HE \& Van Gieson stained. Areas of damage, shown by splicing, were measured using ImageJ, and the ratio of splicing area to vessel area was compared among dilatations (Abb. Po1-09.2).

Results. Umbilical vessel expansion led to parallel splicing, shown by areas devoid of extracellular matrix (ECM) and nuclei, in the tunica intima and media. Abb. Po1-09.1 demonstrates these parallel splicing (indicated by black arrows) in dilated UA and UV in comparison to control, non-dilated vessels. In all vessel expansions, no vertical splicing was observed. Results suggest a threshold expansion of UA $6 \mathrm{~mm}$ and UV $7 \mathrm{~mm}$, as maximal vessel damage was observed above this threshold $(3.6 \pm 2.9 \%$ for UA $7 \mathrm{~mm}$ and $5.2 \pm 2.3 \%$ for UV $8 \mathrm{~mm}$ expansions). Abb. Po1-09.3 shows the percentage of splicing area per vessel for increasing expansion diameter in UA and UV. Conclusion. Loss of vessel integrity may have led to fewer splicing past expansions where maximum splicing was observed (UA $7 \mathrm{~mm}$ and UV $8 \mathrm{~mm}$ ). The thresholds that are suggested for safe expansions (UA $6 \mathrm{~mm}$ and $\mathrm{UV} 7 \mathrm{~mm}$ ) are similar to in utero conditions. Results demonstrate proof of concept for attaining large bore access for the AP. 


\section{Posterwalk 2 - Erstversorgung}

\section{P02-01 \\ Successful treatment of neonatal respiratory transitional disor- der with Pulmo/Vivianit comp. in two cases}

\section{B. Huber'}

'Klinik für Neonatologie, Universitätsspital Zürich, Zürich, Schweiz

Background. Respiratory adaptation comprises the fundamental transition from a hydrospheric to an atmospheric environment at birth, realized by different, highly regulated physiologic changes in the cardiorespiratory system. If these are disturbed or delayed, cardiorespiratory disorders occur, frequently appearing as respiratory distress in the first hours of life. The management of respiratory transitional disorders is focused mainly on respiratory support. In addition to conventional therapies, anthroposophically extended medicine offers the possibility of treatment options based on an integrative approach to man and nature, to illness and healing. The anthroposophic medication Pulmo/Vivianit comp. proved to be effective in the treatment of pulmonary disorders in children and adults without indication of safety problems. Therefore, we hypothesized a possible effect in neonatal respiratory transitional disorder.

Case report 1. A newborn girl presented progressive signs of respiratory distress after birth. Pregnancy, delivery by elective cesarean section at a gestational age of $394 / 7$ weeks and primary adaptation had been normal. After repetitive application of Pulmo/Vivianit comp. orally signs of respiratory distress disappeared within minutes and the girl could stay with the mother under continuous monitoring, that did not reveal abnormal findings until discharge on day five.

Case report 2. Twin boys were delivered by cesarean section at $355 / 7$ weeks of gestation. Only the second twin developed signs of respiratory distress including the need for supplemental oxygen. To support the child's own effort in managing the respiratory transition, Pulmo/ Vivianit comp. was given orally. Within minutes this was followed by a gradual improvement of respiratory symptoms. The sustainable effect was confirmed by normal findings during monitoring and regular examinations until discharge on day seven.

Discussion. Respiratory transitional disorders have a high incidence and there is an urgent need for preventive and therapeutic interventions. Based on the anthroposophic knowledge of human, nature and substance, the treatment with Pulmo/Vivianit comp. supports specifically the transitional changes in the respiratory system at birth. The rapid and sustainable improvement of respiratory symptoms and the overall functional state of the babies in both cases following the application of Pulmo/Vivianit comp. gives reason to be attributed to this specific treatment. No adverse effects could be observed. The idea of specifically supporting the infants own regulative forces in managing the disorder is confirmed by the fact, that the observable development after application of the medication resembles exactly the natural course of the disease, however in an accelerated mode.

Conclusion. According to the encouraging results presented here, the treatment of respiratory tranistional disorders with Pulmo/Vivianit comp. merits further attention in clinical practice and research.

\section{P02-02 \\ Einflussfaktoren auf den PEEP bei der Beutel-Beatmung Neu- geborener}

J. Hartung', G. Schmalisch', C. Roehr ${ }^{2}$

'Charité Universitätsmedizin Berlin, Neonatologie, Berlin, Deutschland, ${ }_{2}^{2}$ John Radcliffe Hospital, Oxford University Hospital Trust, Dept. Neonatology, Oxford, United Kingdom

Hintergrund. Internationale Reanimationsrichtlinien empfehlen bei der manuellen Beatmung Neugeborener PEEP anzuwenden. Bei Verwendung eines selbst-entfaltenden Beatmungsbeutels (SIB) zur Neugebo- renenreanimation sollte daher immer ein PEEP-Ventil angeschlossen sein. Verschiedene Studien konnten jedoch zeigen, dass PEEP-Ventile den eingestellten PEEP nur unzuverlässig aufbauen.

Fragestellung. Ziel der Studie war es, zu untersuchen, welchen PEEP Einweg-PEEP-Ventile verschiedener Hersteller aufbauen können und welchen Einfluss der dabei verwendete Spitzendruck (PIP) und die Atemfrequenz (RR) auf den PEEP haben.

Material und Methoden. Zehn neue Einweg-PEEP-Ventile von 5 verschiedenen Herstellern (je 2 Ventile von Medisize, The Bag, DROH, Vital Signs, Ambu) wurden an einen elektromechanisch angesteuerten SIB angesteckt um ein Modellpüppchen mit einem integrierten Lungenmodell, entsprechend einem $1 \mathrm{~kg}$ Frühgeborenen, zu beatmen. Standardeinstellungen waren: $\mathrm{PIP}=2 \mathrm{O} \mathrm{cmH}_{2} \mathrm{O}, \mathrm{RR}=6 \mathrm{o} / \mathrm{min}$, flow $=8 \mathrm{~L} /$ min, $\mathrm{PEEP}=5$ und $10 \mathrm{cmH} \mathrm{H}_{2} \mathrm{O}$. Um den Einfluss verschiedener Parameter zu testen, wurden außerdem Messungen mit $\mathrm{PIP}=4 \mathrm{O} \mathrm{cmH}_{2} \mathrm{O}$ und $\mathrm{RR}=4 \mathrm{O} / \mathrm{min}$ durchgeführt. Alle Messungen wurden 5-mal wiederholt. Je Messung wurden 10 konsekutive Atemhübe analysiert. Der mit den verschiedenen Einstellungen aufgebaute PEEP wurde mittels eines Beatmungsmonitors ( $\left.\mathrm{CO}_{2} \mathrm{SMO}\right)$ gemessen und anschließend ausgewertet. Ergebnisse. Der Variationskoeffizient der 10 analysierten Atemhübe pro Messung betrug $<2 \%$. Mit den Standardeinstellungen lieferten die PEEP-Ventile im Median (IQR) einen PEEP von 3,12 $(1,63) \mathrm{cmH} 2 \mathrm{O}$ bei Einstellung auf $5 \mathrm{cmH}_{2} \mathrm{O}$ und $5,26(3,46) \mathrm{cmH}_{2} \mathrm{O}$ bei Einstellung auf $10 \mathrm{cmH}_{2} \mathrm{O}$. Die Ventile eines Herstellers konnten nur sehr unzureichend PEEP aufbauen und wurden daher nicht in die folgenden Berechnungen einbezogen. Zwischen den Ventilen der anderen 4 Hersteller gab es statistisch signifikante, aber keine klinisch relevanten Unterschiede im aufgebauten PEEP. Bei Erhöhung des PIP von 20 auf $40 \mathrm{cmH} 2 \mathrm{O}$ nahm der PEEP im Median (IQR) von 2,3 $(0,92)$ auf 3,4 $(1,07)$ $\mathrm{cmH}_{2} \mathrm{O}$ bei einem eingestellten PEEP von $5 \mathrm{cmH}_{2} \mathrm{O}$ bzw. von $4,4(1,54)$ auf $6,1(2,15) \mathrm{cmH}_{2} \mathrm{O}$ bei eingestellten $10 \mathrm{cmH}_{2} \mathrm{O} \mathrm{zu}(\mathrm{p}<0,001)$. Wurde die RR von 40 auf $60 / \mathrm{min}$ erhöht, hatte dies einen Anstieg des PEEP von $2,8(1,16)$ auf $3,4(1,07) \mathrm{cmH}_{2} \mathrm{O}\left(5 \mathrm{cmH}_{2} \mathrm{O}\right.$ eingestellt) bzw. 5,0 $(2,76)$ auf $6,1(2,15) \mathrm{cmH}_{2} \mathrm{O}\left(10 \mathrm{cmH}_{2} \mathrm{O}\right.$ eingestellt) zur Folge $(\mathrm{p}<0,05)$.

Schlussfolgerung. Einweg-PEEP-Ventile am SIB bauen den eingestellten PEEP nicht zuverlässig auf. Der gelieferte PEEP ist u.a. abhängig von der angewandten RR und dem PIP. Anwender sollten Einweg-PEEPVentile vor dem klinischen Einsatz testen und die Variation des PEEP in Abhängigkeit von PIP und RR berücksichtigen.

\section{P02-03}

\section{Die EXIT-Prozedur - eine Option bei der Erstversorgung von Kindern mit Hydrops fetalis mit beidseitigem Hydrothorax}

\section{S. Koch', J. Essers', O. Beringer', F. Reister', H. Hummler', A. Moewes ${ }^{3}$}

'Klinik für Kinder- und Jugendmedizin, Universitätsklinikum Ulm, Sektion Neonatologie und Pädiatrische Intensivmedizin, Ulm, Deutschland, ${ }^{2}$ Universitätsklinikum Ulm, Universitätsfrauenklinik, Ulm, Deutschland, ${ }^{3} \mathrm{St}$. Christopher Hospital of Children, Department of Neonatology, Philadelphia, USA

Hintergrund. Die EXIT(EX utero Intrapartum Treatment)-Prozedur ist ein etabliertes Verfahren der Atemwegssicherung, angewendet bei der Entbindung von Feten mit angeborenen oberen Atemwegsobstruktionen. Sie beinhaltet die teilweise Entwicklung des Kindes durch eine Sectio bei erhaltener Nabelschnurversorgung im geöffneten Uterus. Ziel ist die Aufrechterhaltung der plazentaren Versorgung des Feten bis eine kardiorespiratorische Stabilisierung erreicht ist. In der Literatur ist bisher ein Fallbericht über die Durchführung einer EXIT-Prozedur bei Kindern mit beidseitigen Hydrothoraces beschrieben. Die Sicherung der Atemwege und Entlastung der Pleurahöhlen innerhalb der ersten Lebensminuten ist dabei für den Gasaustausch essentiell. Wir stellen unsere Erfahrungen mit zum Teil adaptiertem EXIT-Verfahren bei 5 Frühgeborenen mit schwerem Hydrops fetalis mit ausgeprägtem beidseitigem Hydrothorax vor.

Fallvorstellungen. Fünf Frühgeborene mit einem Gestationsalter von 29 bis 34 SSW mit ausgeprägtem Polyhydramnion und generalisiertem 
Hydrops fetalis (GG 100-1500 g >97. Perzentile) wurden mit einer EXITProzedur versorgt. In vier Fällen lag ein Chylothorax vor. In vier Fällen erfolgte eine vollständige EXIT-Prozedur mit Intubation und Pleurapunktion unter kontinuierlicher Überwachung durch Pulsoxymetrie bzw. Palpation der Nabelschnur. Die Abnabelungszeiten lagen zwischen 20 und 27 min. In einem Fall erfolgte die Intubation und Abnabelung nach $7 \mathrm{~min}$. Ein Kind starb infolge einer schweren Gasaustauschstörung bei Lungenhypoplasie nach wenigen Lebensstunden. Ein Kind konnte nach 4 Wochen mit unauffälligem Untersuchungsbefund entlassen werden, die übrigen 3 Kinder sind derzeit noch in unserer stationären Behandlung.

Diskussion. Bei ausgeprägtem Hydrops fetalis mit beidseitigem Hydrothorax sind aufgrund massiver Ödeme relevante Schwierigkeiten bei der Atemwegssicherung zu erwarten. Durch Intubation und Entlastung der Pleurahöhlen unter erhaltener uteroplazentarer Perfusion kann eine postnatale Hypoxämie des Neugeborenen vermieden werden. Voraussetzung ist die Aufrechterhaltung der uterofetalen Perfusion durch ein multidisziplinäres Team aus Neonatologie, Geburtshilfe und Anästhesie. Hauptrisiko für die Mutter ist eine Hämorrhagie infolge vorzeitiger Plazentalösung. Eine EXIT-Prozedur kann bei der Erstversorgung von Kindern mit ausgeprägtem Hydrops mit zu erwartenden Schwierigkeiten bei der Sicherung der Atemwege unter Beachtung der damit verbundenen Risiken für Mutter und Kind erwogen werden.

\section{P02-04}

\section{Entwicklung und praktische Umsetzung eines simulations- basierten interdisziplinären Ausbildungskonzeptes in der Neugeborenenreanimation}

\section{Mileder', J. Pansy', M. Pocivalnik', W. Raith', B. Urlesberger' \\ 'Univ.-Klinik für Kinder- und Jugendheilkunde, Medizinische Universität Graz, Klinische Abteilung für Neonatologie, Graz, Österreich}

Hintergrund. Die umfassende kardiopulmonale Reanimation von Neugeborenen ist ein seltenes, jedoch hochkritisches Ereignis. Aufgrund der limitierten klinischen Ausbildungs- und Trainingsmöglichkeiten empfehlen internationale Reanimationsrichtlinien daher die Nutzung simulationsbasierter Ausbildungsmethoden.

Fragestellung. Zielsetzung waren Konzeption und praktische Umsetzung einer eintägigen Fortbildungsveranstaltung in der Neugeborenenreanimation für das medizinische Personal einer geburtshilflichen Privatklinik

Material und Methoden. Das Ausbildungskonzept wurde nach folgenden didaktischen Gesichtspunkten entwickelt:

1) Adaptierung der Inhalte an die Bedürfnisse des Zielpublikums (GynäkologInnen, AnästhesistInnen, Hebammen, Diplompflegepersonal), 2) Maximierung der individuellen praktischen Trainingsmöglichkeiten im interdisziplinären Team durch Trainer-Trainee-Verhältnis von maximal 1:5,

3) repetitive Anwendung aktueller Reanimationsrichtlinien und

4) hohe Realitätsnähe des Trainings durch Nutzung lokaler Ressourcen. Als Surrogatparameter für die Effektivität der Fortbildungsveranstaltung wurde nach Absolvierung die Zufriedenheit der TeilnehmerInnen mittels eines Paper-and-pencil-Evaluierungsbogens (geschlossene Fragen mit 3-teiligen Skalen und Freitextkommentare) erhoben.

Ergebnisse. Die insgesamt 7,5-stündige Fortbildungsveranstaltung setzte sich wie folgt zusammen:

- 2 interaktive Vorträge über Grundlagen und spezielle Situationen der Neugeborenenreanimation (Gesamtdauer 6o Minuten)

- 1 Workshop zu Fertigkeiten des Atemwegsmanagements (u. a. Indikation, Beatmung mit Beatmungsbeutel/Beatmungsgerät mit T-Stück, Intubation, Sauerstofftherapie; Gesamtdauer 45 Minuten)

- 1 Workshop zu Gefäßzugängen (intravenöser/intraossärer Zugang, Nabelvenenkatheter) und Medikamenten (Gesamtdauer 45 Minuten)

- 4 Simulationseinheiten, im Rahmen derer 16 klinische Szenarien praxisnahe trainiert wurden (Gesamtdauer: 18o Minuten).
Workshops und Simulationseinheiten fanden in Kleingruppen zu jeweils maximal sechs TeilnehmerInnen statt. Sechzehn Personen (9 ÄrztInnen, 6 Hebammen, 1 Diplompflegefachkraft) nahmen an der Fortbildungsveranstaltung teil. Vierzehn Evaluierungsbögen $(87,5 \%)$ wurden retourniert. Die allgemeine Zufriedenheit mit der Fortbildungsveranstaltung war sehr hoch (Bewertung „sehr gut“: n=14/14). Der am häufigsten genannte positive Aspekt waren der Praxisbezug bzw. das intensive praktische Training $(\mathrm{n}=9 / 14)$. Die regelmäßige Wiederholung der Fortbildungsveranstaltung wurde als häufigster Verbesserungsvorschlag angeführt $(\mathrm{n}=8 / 9)$.

Diskussion. Das präsentierte Ausbildungskonzept konnte erfolgreich umgesetzt werden und kann als Vorlage für die simulationsbasierte Aus- und Weiterbildung von interdisziplinären medizinischen Teams in der praktischen Durchführung der Neugeborenenreanimation dienen. Die inhaltliche und didaktische Konzeption ermöglichen die repetitive praktische Anwendung des in prägnanten theoretischen Einheiten erworbenen Wissens in realitätsnaher Umgebung.

\section{P02-05}

Einfluss der Hand(schuh)größe des medizinischen Personals und der Beatmungstechnik (2-Finger- vs. 5-Finger-Technik) auf das Tidalvolumen bei Beutelmaskenbeatmung am Säuglingsphantom

\section{H. Kilbertus', B. Urlesberger', B. Schwaberger}

'Medizinercorps Graz, Österreichisches Rotes Kreuz Landesverband Steiermark, Klinische Abteilung für Neonatologie, Universitätsklinik für Kinderund Jugendheilkunde, Medizinische Universität Graz und, Graz, Österreich, ${ }^{2}$ Medizinische Universität Graz, Klinische Abteilung für Neonatologie, Universitätsklinik für Kinder- und Jugendheilkunde, Graz, Österreich

Hintergrund. Internationale Leitlinien zur Reanimation von Säuglingen betonen die Wichtigkeit einer effektiven Beutelmaskenbeatmung. Die Effektivität soll dabei durch die Beobachtung von Thoraxexkursionen, die Überwachung der Herzfrequenz und Sauerstoffsättigung sowie durch Auskultation von Atemgeräuschen erfolgen. Es werden keine Angaben darüber gemacht, mit welcher Technik man effektive Tidalvolumen erzielen kann. Der Einfluss der Hand- bzw. Handschuhgröße des Beatmenden auf das abgegebene Tidalvolumen wurde bisher nicht untersucht.

Fragestellung. Haben die Hand- bzw. Handschuhgröße des medizinischen Personals oder die Beatmungstechnik (2-Finger- vs. 5-FingerTechnik) einen Einfluss auf das Tidalvolumen bei der Beutelmaskenbeatmung am Säuglingsphantom?

Material und Methode. 40 Probanden aus medizinischen Berufsgruppen wurden rekrutiert (20 Rettungssanitäter und 20 diplomierte Kinderkrankenschwestern). Ein Säuglingsphantom (CPR Resusci Baby, Laerdal; Norwegen) wurde so adaptiert, dass eine leckfreie Testlunge (Dräger 0,5 L) in die Puppe eingebaut wurde. Die Probanden wurden aufgefordert, das Säuglingsphantom mit Beatmungsbeutel (Ambu Baby-R, Ambu; Dänemark) und einer Silikonmaske rund Größe o/1 (Laerdal; Norwegen) effektiv zu beatmen. Zur Beurteilung der Suffizienz der Beatmung fungierten die Thoraxexkursionen der Reanimationspuppe. Pro Studienteilnehmer wurden zwei Messdurchgänge zu 9o Sekunden durchgeführt: 2-Finger-Technik (Daumen und Zeigefinger) und 5-Finger-Technik. Tidalvolumen und Maskenleck wurden mit einem Florian Respiratory Function Monitor (Acutronic; Schweiz) gemessen.

Ergebnisse. Hand- bzw. Handschuhgröße der Probanden korrelierten nicht mit dem applizierten Tidalvolumen $(p=0,70)$. Das mittlere abgegebene Tidalvolumen bei 2- bzw. 5-Finger-Technik zeigte keinen signifikanten Unterschied $(\mathrm{p}=\mathrm{o}, 22)$; das mittlere Maskenleck war allerdings bei der 5 -Finger-Technik signifikant höher $(\mathrm{p}=0,02)$. Zwischen Rettungssanitätern und diplomierten Kinderkrankenschwestern bestanden bei der Beutelmaskenbeatmung hinsichtlich der abgegebenen Tidalvolumen $(\mathrm{p}=0,55)$ und des Maskenlecks $(\mathrm{p}=0,32)$ keine signifikanten Unterschiede; Rettungssanitäter führten die Beatmung mit einer signifikant niedrigeren Frequenz durch $(\mathrm{p}<0,001)$. 
Schlussfolgerung. Bei medizinischem Personal ist eine effektive Beutelmaskenbeatmung am Säuglingsphantom sowohl mit 2- als auch mit 5-Fingertechnik möglich und ist unabhängig von der Hand(schuh)größe des Beatmenden. Beide Erkenntnisse könnten zukünftig in Reanimationsleitlinien erwähnt werden, um diesbezügliche Unsicherheiten bei der Beutelmaskenbeatmung zu beseitigen.

\section{P02-06}

\section{Videoanalyse der Erstversorgung von Frühgeborenen}

\section{Konstantelos', J. Dinger', M. Mögel', S. Ifflaender', M. Rüdiger'}

'Medizinische Fakultät Carl Gustav Carus an der TU Dresden, Fachbereich Neonatologie und Pädiatrische Intensivmedizin, Dresden, Deutschland

Hintergrund. In den vergangenen Jahren fokussierte sich die Forschung bezogen auf die Erstversorgung Frühgeborener auf die Wirksamkeit einzelner Interventionen. Wenig Beachtung fand dabei die die Prozessqualität der gesamten Erstversorgung. Zielstellung. Detaillierte Analyse des Status quo der Erstversorgung Frühgeborener in einem Perinatalzentrum Level 1 unter besonderer Berücksichtigung der Beachtung interner Leitlinien und Variationen im Management der einzelnen Patienten.

Material und Methode. Die Erstversorgung per Sectio Frühgeborener wird im Frühdienst routinemäßig im Rahmen eines Qualitätssicherungsprojektes videographiert. Die in den Jahren 2012 und 2013 aufgezeichneten Videos wurden mittels Interact-Software wie folgt ausgewertet: Anzahl, Zeitpunkt, Dauer und Reihenfolge verschiedener medizinischer Interventionen.

Ergebnisse. 111 Erstversorgungen [ $33+5$ SSW $(26+1-36+6)$, Mediane Video-Dauer: 24, Range: 10-57 Minuten] wurden analysiert, 90\% der Frühgeborenen wurden nach der Erstversorgung auf die neonatologische Intensivstation verlegt. Alle Kinder wurden auskultiert [Start nach 8 (2-93) Sek.] und pulsoximetrisch überwacht [Start 37 (12-123) Sek.]. $91(83 \%)$ der Neugeborenen erhielten eine Atemunterstützung [Start 28 Sek. (2-1461), Dauer 10 (0,9-40) Minuten]. 51\% der Neugeborenen wurden für 8 (2-20) Sekunden gebläht [Start 0,2 (1,9-7,3) Minuten]. 23 Frühgeborene wurden nach 9 (1-50) Minuten intubiert und 17 erhielten Surfactant, 29\% wurden anschließend extubiert. Die Dauer der Intubationen lag bei 47 (7-125) Sekunden. Ein IV-Zugang wurde nach 15 (0,5-41) Minuten gelegt, die dafür benötigte Zeit betrug 3 (1-7) Minuten. Diskussion. Die Mehrzahl der Frühgeborenen erhielt unmittelbar nach der Geburt eine Atemunterstützung; sowohl Dauer als auch Startpunkt der einzelnen Maßnahmen zeigten große Unterschiede innerhalb der untersuchten Patientenpopulation. Das Monitoring der Kinder erfolgte entsprechend der lokalen Therapieempfehlungen.

Schlussfolgerung. Die Videoanalyse als Instrument der Qualitätssicherung eignet sich zur Beschreibung klinischer Behandlungsprozesse; interindividuelle Unterschiede und Abweichungen von internen Therapieempfehlungen können aufgezeigt werden. Diese Daten können Ansätze für gezielte Qualitätsverbesserung aufzeigen und für ein Benchmarking mit anderen Einrichtungen genutzt werden.

\section{P02-07 \\ Video Apgar Trial: Vergleich des Vorgehens bei der Erstversor- gung in 3 europäischen Ländern}

S. Walter', S. Burkhard', J. van Vonderen ${ }^{3}$, D. Konstantelos' ${ }^{4}$, A. te Pas ${ }^{3}$, H. Küster ${ }^{1}$ Neonatologie, Universitäts-Kinderklinik, Göttingen, Deutschland, ${ }^{2}$ Kinderklinik Feldkirch, Feldkirch, Österreich, ${ }^{3}$ Neonatology, University Medical Center Leiden, Leiden, Niederlande, ${ }^{4}$ Fachbereich Neonatologie und Pädiatrische Intensivmedizin, TU Dresden, Dresden, Deutschland

Hintergrund. Die Erstversorgung im Kreißsaal unterliegt seit einigen Jahren einem erheblichen Wandel. Es gibt sowohl lokale wie nationale Leitlinien, die - teils auf wissenschaftlicher Basis - eine Vereinheitlichung des Vorgehens zum Ziel haben.
Ziel. Objektive Erfassung und Beschreibung des Vorgehens bei der Erstversorgung von Frühgeborenen mit einem Geburtsgewicht $<1500 \mathrm{~g}$ oder $<32$ SSW (VLBW) in 4 Perinatalzentren in 3 europäischen Ländern. Methodik. Im Rahmen des prospektiven Video Apgar Trial wurden in großen Level 1 Perinatalzentren anhand eines standardisierten Protokolls Videos von Erstversorgungen aufgenommen. Jetzt konnten Videos von 4 Perinatalzentren von einer Person, die in keinem dieser Zentren in die Versorgung der Frühgeborenen involviert war, anonym nach einem standardisierten Schema ausgewertet werden. 48 Parameter wurden zwischen den Zentren verglichen und mit dem Outcome korreliert.

Ergebnisse. Bis jetzt wurden 137 Videos ausgewertet (26/42/43/27). Das mittlere Gestationsalter der gefilmten Frühgeborenen betrug 29 SSW, das mittlere Geburtsgewicht $1231 \mathrm{~g}$. Die Zeit im Erstversorgungsraum variierte signifikant zwischen den Zentren (25/25/13/28 Minuten). Während Absaugen in einem Zentrum deutlich häufiger beobachtet wurde als in den anderen (Mittel o,4/2,5/o,6/1,5-mal pro Kind), war die Dauer der einzelnen Absaugungen vergleichbar (Mittel 10 s). Die Zeit zwischen Anlegen des Sättigungssensors und dem ersten korrekten Signal unterschied sich deutlich (44/20/22/61 s). Die Folie, in die das Frühgeborene eingewickelt war, wurde im Mittel 13/10/5/14-mal rearrangiert. Nur 29/27/44/20\% der Zeit im Erstversorgungsraum wurde an dem Frühgeborenen keine Manipulation beobachtet. Auch bei der Häufigkeit der Temperaturmessung oder der Dauer und Häufigkeit von Atemhüben und Stimulation fanden sich zwischen den Zentren Unterschiede. Über die Dauer der Studie war eine Verbesserung der Versorgungsqualität zu erkennen im Sinne eines zielgerichteten und einheitlichen Vorgehens. Eine Korrelation zwischen Vorgehen im Kreißsaal und den typischen neonatologischen Outcomeparametern bestand nicht.

Schlussfolgerung. Videoaufnahmen im Kreißsaal sind ein wirkungsvolles Werkzeug zur Vereinheitlichung des Vorgehens bei der Erstversorgung und schon allein dadurch auch zur Qualitätsverbesserung. Manche Ergebnisse der Auswertung waren für alle Beteiligten überraschend, insbesondere die Häufigkeit an Manipulationen. Auch wenn das Vorgehen durch lokale Gegebenheiten und Grundvorstellungen beeinflusst wird, finden sich zwischen den Zentren Unterschiede im Vorgehen, die damit allein nicht erklärt werden können. Nicht an allen Stellen sind Empfehlungen und Leitlinien umgesetzt, diese sind aber bezüglich des Zeitablaufs auch unrealistisch.

\section{P02-08 \\ Vergleich prä-und postnataler Prognoseparameter bei angebo- rener Zwerchfellhernie}

\section{T. Schaible', S. Reschke', W. Neff', S. Hien ${ }^{4}$, R. Schaffelder', L. Wessel ${ }^{4}$}

${ }^{1}$ Klinikum Mannheim, Klinik für Neonatologie, Mannheim, Deutschland, ${ }^{2}$ Universitätsklinikum Mannheim, Institut für Klinische Radiologie, Mannheim, Deutschland, ${ }^{3}$ Klinikum Mannheim, Kinderklinik, Mannheim, Deutschland, ${ }^{4}$ UMM Mannheim, Kinderchirurgie, Mannheim, Deutschland

Hintergrund. Die kernspintomografische Messung des relativen fetalen Lungenvolumens (rFLV) ist ein potenter pränataler Prognoseparameter hinsichtlich des postnatalen Outcomes bei der angeborenen Zwerchfellhernie. Ob die Wertigkeit postnataler Prognosekriterien in einem homogenen Kollektiv mit standardisierter Versorgung an einem hochspezialisierten Zentrum die Prognoseprädiktion bestätigt, war Gegenstand der vorliegenden Analyse.

Patienten und Methodik. In den Jahren 2008 bis 2012 wurden bei 100 $\mathrm{CDH}$ Patienten das rFLV und 3 postnatale Prognosefaktoren hinsichtlich der Outcomeparameter Mortalität, Bedarf für eine Behandlung mittels der extracorporalen Membranoxygenierung (ECMO) und des Auftretens einer mittleren oder schweren chronischen Lungenerkrankung (CLD) ausgewertet. Ausgeschlossen wurden Frühgeborene unter $34+0$ Schwangerschaftswochen sowie Patienten mit schweren assoziierten Fehlbildungen oder Zustand nach fetaler Intervention. Postnatal wurde der SNAPPE II Score in den ersten 6-12 Lebensstunden, die Wilford Hall /Santa Rosa prediction formula (WHSR) bestehend aus 
dem besten präduktalen Blutgasparametern $\mathrm{paO}_{2}$ minus $\mathrm{paCO}_{2}$ in den ersten 6-12 Stunden sowie der einfache CDH Study Group Score (CDHSG), welcher nur Reife, Geburtsgewicht und den 5 min APGAR misst. ermittelt.

Ergebnisse. Die Mortalität betrug lediglich 11\%. Hierfür fanden sich für alle Scores signifikante Unterschiede im Vergleich zu den Überlebenden. Die pränatal exzellente Prädiktion des rFLV mit einer AUC von o,82 wurde vom postnatalen SNAPPE II mit einer AUC von 0,85 und vom einfachen CDHSG Score mit o,87 übertroffen. 36 Patienten erhielten eine ECMO Therapie. Hinsichtlich des ECMO Bedarfs fand sich die beste AUC mit 0,89 für die WHSR, der CDHSG Score ist nicht zur Vorhersage einer ECMO geeignet (AUC 0,56). Auch die CLD kann mit allen Scores relativ gut vorhergesagt werden, die AUCs bewegen sich hierbei zwischen 0,76 und o,84 mit der WHSR Formel als bestem Prädiktor. Schlussfolgerung. Unter dem Setting eines spezialisierten Zentrums mit einem standardisierten Therapiealgorithmus bestätigen und ergänzen die postnatalen Prognosescores die pränatale Vorhersage mit großer Genauigkeit. Noch vor einer ECMO Therapie und vor einer Operation besteht somit eine größere Therapiesicherheit für die CDH Patienten

\section{P02-09}

\section{Lungenbelüftung bei den ersten Atemzügen in einem Kanin- chenmodell der Zwerchfellhernie}

\section{A. Flemmer', M. Wallace ${ }^{2}$, M. Kitchen ${ }^{3}$, M. Thio Lluch', C. Roehr ${ }^{5}$, J. Jani ${ }^{6}$, M. Siew', K. Lee', G. Buckley', N. Yagi ${ }^{8}$, K. Uesugi ${ }^{8}$, S. Hooper ${ }^{9}$}

'LMU-München, Neonatologie der Kinderklinik am Perinatalzentrum Großhadern, München, Deutschland, ${ }^{2}$ The Ritchie Centre, Monash Institute of Medical Research, Melbourne, Australien, ${ }^{3}$ School of Physics, Monash University, Melbourne, Australia, Melbourne, Australien, ${ }^{4}$ The Royal Women's Hospital, Melbourne, Australien, Division of Neonatal Research, Parkville $\mathrm{VIC}^{3052}$, Australien, ${ }^{5}$ Charité Medical Center Berlin, Dept. Neonatology, Berlin, Deutschland, ${ }^{6}$ University Hospital Brugmann, Brussels, Belgium, Fetal Medicine Unit, Brüssel, Belgien, ${ }^{7}$ School of Physics, Monash University, Melbourne, Australia;, Melbourne, Australien, ${ }^{8}$ Spring $-{ }^{8}$ Synchrotron, JASRI, Hyogo, Japan, Hyogo, Japan, ${ }^{9}$ Department of Physiology, Monash Institute for Medical Resarch, Monash University, Melbourne, Australien

Hintergrund. Die kongenitale Zwerchfellhernie (DH) kommt bei ca. 1:400o Neugeborenen vor und ist auch heute noch mit einer hohen Mortalität bel astet, wenn die durch die Herniation von Bauchorganen induzierte Lungenhypoplasie ausgeprägt ist. Die aktuellen Empfehlungen zum initialen respiratorischen Management dieser Kinder basieren im Wesentlich auf Expertenmeinungen und es existieren weder klinische noch experimentelle Daten, die den Effekt der initialen Beatmung auf die Gasverteilung in der inhomogenen Lunge untersuchen. Ziel dieser Studie war es, die Gasverteilung während der ersten Atemzüge in einem Kaninchenmodell der Zwerchfellhernie zeitlich und räumlich zu untersuchen.

Methode. Fetale Kaninchen wurden am 25/31 Gestationstag an der uteroplazentaren Perfusion exponiert und in einer Ex-utero-Operation eine linksseitige Zwerchfellhernie angelegt. Im Anschluss wurde die Schwangerschaft bis zum Termin erhalten und Hernien-Feten (DH; $\mathrm{n}=6)$ sowie nicht operierte Kontrollen $(\mathrm{Ko} ; \mathrm{n}=8)$ per Sektio in Vollnarkose entbunden. Vor dem ersten Atemzug wurden die Feten in den Strahlengang eines Synchrotron-Röntgen Detektors (Spring-8, Osaka, Japan) in einem Körperplethysmographen eingebracht. Die ersten Atemzüge wurden volumenkontrolliert $(5 \mathrm{ml} / \mathrm{kg}$ ) bei einem PEEP von $5 \mathrm{cmH}_{2} \mathrm{O}$ appliziert. Die Luftfüllung der Lungen wurde mit Hilfe der Phasenkontrast Röntgen Technik $(25 \mathrm{keV})$ mit $5 \mathrm{~Hz}$ bei $20 \mathrm{~ms} /$ frame aufgezeichnet und hinsichtlich räumlichen und zeitlichen Verlauf ausgewertet. Am Ende der Beatmung wurde die Lungen-Körper-Ratio (LBWR) bestimmt.

Ergebnisse. Die Überlebensrate der operierten Feten lag bei 58\%. Es zeigte sich kein Unterschied zwischen DH- und Ko-Feten hinsichtlich des Körpergewichtes. Die LBWR war bei DH-Feten signifikant geringer

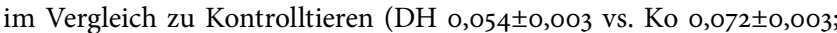

$\mathrm{p}<0,0005)$. In DH-Feten füllte sich die rechte Lunge dreimal schneller im Vergleich zur linken Lunge $(1,083 \pm 0,388$ vs. $0,378 \pm 0,192 \mathrm{ml} / \mathrm{min}$; $\mathrm{p}<0,05)$. Die Luftfüllung der rechten $\mathrm{DH}$-Lunge war geringer und langsamer im Vergleich zu Ko-Lungen. Bei DH-Feten wurde die FRC rechts schneller als links erreicht $(0,747 \pm 0,116 \mathrm{ml}$ vs. $0,423 \pm 0,057 \mathrm{ml} ; \mathrm{p}<0,05)$ und lang insgesamt unter den Ko-Lungen.

Schlussfolgerung. Bei inhomogenen DH-Lungen besteht ein erhöhtes Risiko der Überblähung der kontralateralen Lunge bei noch atelektatischen Lunge der ipsilateralen Seite. Unsere Daten zeigen, dass mittels Echtzeit-Phasenkontrast-Röntgen die regionale und zeitliche Belüftung der Lunge in den ersten Atemzügen bei Zwerchfellhernie dargestellt und ausgewertet werden kann. Damit kann diese Methode neue Erkenntnisse in Hinblick auf die optimale initiale Beatmung von Kindern mit $\mathrm{CDH}$ erbringen.

\section{P02-10}

Zentrale und periphere Hauttemperatur am ersten Lebenstag im Vergleich zwischen konventioneller Bettung mit Säuglingsoberbett zusätzlich zum Schlafsack und Bettung mit Schlafsack bei eutrophen Neugeborenen

\section{Hoffmann', A. Stein', U. Felderhoff-Müser ${ }^{2}$}

'Klinik für Kinder und Jugendmedizin der Universität, Klinik für Kinderheilkunde I, Essen, Deutschland, ${ }^{2}$ Universitätsklinikum Essen, Direktorin der Klinik für Kinderheilkunde I, Zentrum für Kinder- und Jugendmedizin, Essen, Deutschland

Einleitung. Zur Prophylaxe des Plötzlichen Kindstodes (SIDS) wird zur Bettung Neugeborener ein Schlafsack empfohlen. In der direkt postnatalen stationären Betreuung wird dies in vielen Kliniken aus Sorge vor Hypothermie nicht umgesetzt. Dies erschwert das elterliche Verständnis für eine konsequenten Umsetzung der empfohlenen Schlafumgebung nach Entlassung.

Ziel. Vergleich zweier Bettungsmethoden bei reifen eutrophen Neugeborenen am ersten Lebenstag mit dem Ziel, das tatsächliche Risiko einer Hypothermie am ersten Lebenstag aufgrund der alleinigen Anwendung eines Schlafsacks sowie das Risiko einer Hyperthermie durch Verwendung einer Bettdecke zu evaluieren.

Methoden. Randomisierte prospektive Fall-Kontroll-Studie bei 40 eutrophen gesunden Neugeborenen in einer Universitätsklinik. Es wurden Hauttemperaturen an drei Messpunkten in zwei Gruppen (alleiniger Schlafsack versus Bettdecke) über einen Zeitraum von mindestens 8 Stunden sowie eine abschließende Rektaltemperatur erhoben mit einer Subgruppenanalyse in verschiedenen Gewichtsklassen - leicht (10.-30. Perzentile), mittel (30.-70. Perzentile) und schwer (70-90. Perzentile). Ergebnisse. Bei den im Median gemessenen Hauttemperaturen an Stirn, Sternum und Hand bestehen signifikante Differenzen zwischen Bettung im Schlafsack (S) oder mit Decke (D). Während im Schlafsack an Stirn und Sternum höhere Temperaturen erreicht werden (Stirn $31,9 \pm 0,1^{\circ} \mathrm{C}$ vs. $31,3 \pm 0,4^{\circ} \mathrm{C} ; \mathrm{p}=0,000$; Sternum $36,0 \pm 0,1^{\circ} \mathrm{C}$ vs. $35,8 \pm 0,1^{\circ} \mathrm{C}$; $\mathrm{p}=0,000)$ liegen die an der Hand gemessenen Temperaturen bei Lagerung mit Bettdecke höher $\left(26,9 \pm 0,4^{\circ} \mathrm{C}\right.$ vs. $\left.28,7 \pm 0,4^{\circ} \mathrm{C} ; \mathrm{p}=0,000\right)$. Die erreichten Minimal- und Maximaltemperaturen unterscheiden sich an allen 3 Messpunkten nicht signifikant. Auch die am Ende der Messdauer erhobenen Rektaltemperaturen unterscheiden sich nicht signifikant

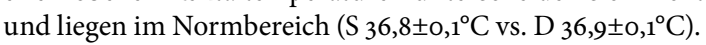

Schlussfolgerung. Trotz teilweise signifikanter Unterschiede zwischen den im Median erreichten Hauttemperaturen bei unterschiedlichen Bettungsmethoden und Gewichtsklassen trat bei keinem Kind eine rektal gemessene Hypo- oder Hyperthermie auf. Unsere Studie konnte die Annahmen widerlegen, dass eine zusätzliche Bettdecke automatisch zu höheren Temperaturen führt. Lediglich die Hände, die meist unter der Decke lagen, wiesen eine höhere Temperatur auf. Somit kann die risikoärmere Bettung im Schlafsack von Geburt an für reife eutrophe Neugeborene empfohlen werden. 
P02-11

Die Aufnahmetemperatur bei sehr unreifen Frühgeborenen ist assoziiert mit der Sterblichkeit: Ergebnisse der EPICE-Studie

R. Maier', E. Wilson'2, M. Norman², B. Misselwitz'3 , J. Zeitlin', A. Edstedt Bonamy² 'Universitätsklinikum Marburg, Klinik für Kinder- und Jugendmedizin, Marburg, Deutschland, ${ }^{2}$ Karolinska Institutet, Solna/Stockholm, Deutschland, ${ }^{3}$ Geschäftsstelle Qualitätssicherung Hessen, Eschborn, Deutschland, ${ }^{4}$ Inserm, Paris, Frankreich

Hintergrund. Eine Unterkühlung gilt seit langem als Risikofaktor für eine ungünstige Prognose bei Frühgeborenen. Strategien zur Verhinderung einer Unterkühlung, wie der Gebrauch von Plastikbeuteln oder Wärmematten, haben sich in randomisierten kontrollierten Studien als wirksam erwiesen.

Hypothese. Eine Hypothermie bei Aufnahme auf eine neonatologische Intensivstation kommt bei sehr unreifen Frühgeborenen in Europa immer noch häufig vor und ist mit einer erhöhten Sterblichkeit assoziiert. Methodik. Im Rahmen des EPICE-Projektes wurden in den Jahren 2011 bis 2012 über einen Zeitraum von 12 Monaten in 19 Regionen in 11 europäischen Ländern alle lebendgeborenen Kinder mit einem Gestationsalter von 22+o bis $31+6$ SSW erfasst. Der Zusammenhang zwischen der Temperatur bei Aufnahme auf eine neonatologische Intensivstation und der Mortalität wurde mittels logistischer Regression analysiert. Das endgültige logistische Regressionsmodell war adjustiert für Gestationsalter, Geburtsgewicht, Apgar $<7$ Punkte mit 5 Minuten, Geschlecht und Geburtsregion. Eine Hypothermie wurde definiert als Körpertemperatur $<36,0^{\circ} \mathrm{C}$.

Ergebnisse. Von den 7483 auf eine neonatologische Intensivstation aufgenommenen Frühgeborenen wurde bei 6561 Kindern die Aufnahmetemperatur dokumentiert. Bei 1656 (25\%) Kindern bestand eine Hypothermie, wobei die regionalen Unterschiede in der Rate unterkühlter Kinder erheblich waren. Die Aufnahmetemperatur war negativ korreliert mit der Mortalität. Im Vergleich zu Frühgeborenen mit einer Aufnahmetemperatur von $36,5-37,4^{\circ} \mathrm{C}$ war die Odds Ratio (95\% Konfidenzintervall) für Mortalität $5,88(4,52-7,65)$ bei Frühgeborenen mit einer Temperatur $<35,0^{\circ} \mathrm{C}, 3,25(2,46-4,30)$ bei Kindern mit $35,0-35,4^{\circ} \mathrm{C}$ und $1,73(1,32-2,27)$ bei Kindern mit $35,5-35,9^{\circ} \mathrm{C}$. Auch im adjustierten Modell war eine Aufnahmetemperatur $<35,5^{\circ} \mathrm{C}$ assoziiert mit erhöhter Sterblichkeit: OR 2,09 $(1,48-2,94)$ bei Kindern mit einer Temperatur $<35,0^{\circ} \mathrm{C}$ und OR $1,89(1,32-2,72)$ bei Kindern mit 35,0-35, $4^{\circ} \mathrm{C}$, jeweils im Vergleich mit normothermen Kindern. Ein Zusammenhang zwischen Hypothermie und Sterblichkeit bestand sowohl in der ersten Lebenswoche als auch in der weiteren Neonatalperiode.

Schlussfolgerung. Trotz der bekannten Risiken einer Hypothermie und trotz vorhandener Strategien zu ihrer Verhinderung ist die Hypothermie bei sehr unreifen Frühgeborenen in Europa immer noch ein häufiges Phänomen und ist assoziiert mit erhöhter Sterblichkeit. In weiteren Studien sollte untersucht werden, inwieweit die Evidenz-basierten Strategien zur Verhinderung einer Hypothermie auch tatsächlich eingesetzt werden.

Mit Unterstützung der Europäischen Union (7. Rahmenprogramm).

\section{Posterwalk 3 - Immunologie}

P03-01

Clinoleic hemmt die entzündungsbedingte Leukozytenrekrutierung und verbessert das Überleben in der LPS-induzierten Endoxämie

K. Buschmann', J. Pöschl', N. Braach', N. Kuss', H. Hudalla' , D. Frommhold'

'Universitätsklinikum Heidelberg, Zentrum für Kinder- Jugendmedizin, Klinik Kinderheilkunde IV/Neonatologie/ Angelika-Lautenschläger-Klinik, Heidelberg, Deutschland

Hintergrund. Parenterale Ernährung beeinflusst die unspezifische Abwehr und die Funktion von neutrophilen Granulozyten. In den letzten Jahren wurden insbesondere fischöl- und olivenölhaltigen Lipidkompositionen antiinflammatorische Eigenschaften zugeschrieben.

Fragestellung. Es wurde die immunmodulatorische Wirkung von mittel- und langkettigen Fettsäuren (MCT bzw. LCT), fisch- und olivenölhaltigen Lipidinfusionen auf die Leukozytenrekrutierung in verschiedenen Entzündungsmodellen der Maus untersucht.

Material und Methoden. Nach intravenöser Applikation wurde intravitalmikroskopisch der Einfluss von Lipofundin (je 50\% LCT/MCT), Smof-Lipids ( $15 \% \mathrm{O}_{3}$ Fettsäuren, je 30\% MCT/LCT, 25\% Olivenöl) und Clinoleic (je 50\% Olivenöl/LCT) auf die Trauma- und LPS-induzierte Leukozytenadhäsion untersucht. Zudem wurde der Einfluss der genannten Fette im LPS-induzierten Endotoxinämie-Modell evaluiert. Diese Versuche wurden durch die Untersuchung der Leukozytenadhäsion in dynamischen Flusskammern und Expressionsanalysen von Adhäsionsmolekülen ergänzt.

Ergebnisse. Nach intravenöser Gabe von $1 \mathrm{~g} / \mathrm{kg}$ Clinoleic wurden die Trauma- und die LPS- induzierte Leukozytenrekrutierung signifikant gehemmt. Dieser antiinflammatorische Effekt war in geringerem Maße auch nach Infusion von Smof-Lipids, nicht allerdings von Lipofundin zu verzeichnen. Mit Clinoleic behandelte Mäuse zeigten ferner einen signifikanten Überlebensvorteil im LPS-SIRS- Modell. Die LPS-induzierte Leukozytenadhäsion in der Mikroflusskammer konnte ebenfalls durch Clinoleic, jedoch nicht durch Smof-Lipids bzw. Lipofundin reduziert werden. In diesem Zusammenhang beobachteten wir durchflusszytometrisch eine endotheliale Downregulation von VCAM-1 nach Clinoleic und Smof-Lipids. Die leukozytäre Expression von CXCR2 sank deutlich nach Clinoleic und Smof-Lipids, während die PSGL1-Expression ausschließlich nach der Applikation von Clinoleic reduziert wurde. Die $\beta 2$-Integrinexpression blieb von der Lipidbehandlung weitestgehend unbeeinflusst, wobei eine vermehrte Expression von MAC1 nach der Applikation von Lipofundin zu beobachten war.

Diskussion. Das Olivenöl-haltige Clinoleic entfaltete am stärksten antiinflammatorische Effekte im Sinne einer Hemmung der Leukozytenrekrutierung und Verbesserung des Überlebens im LPS-SIRS Modell. Während nach Lipofundin keine Auswirkungen auf die Leukozytenadhäsion beobachtet wurden, zeigte sich nach Smof-Lipids ein moderater antiinflammatorischer Effekt. Clinoleic erschien hierbei den Smof-Lipids überlegen. Die weitere mechanistische Abklärung durch Flusskammerexperimente und FACS-Analysen deutet auf eine Clinoleic-induzierte Downregulation sowohl leukozytärer als auch endothelialer Adhäsionsmoleküle hin. Künftige Studien sollten klären, ob die Gabe von Clinoleic bei kritisch kranken Patienten als supportive Therapie entzündlicher Erkrankungen sinnvoll erscheint.

Schlussfolgerung. Auf LCT und Olivenöl basierendes Clinoleic bewirkt in verschiedenen Maus-Entzündungsmodellen einen signifikanten antiinflammatorischen Effekt. 


\section{P03-02}

Progesteron steigert die suppressive Aktivität myeloider Suppressorzellen (MDSC) an der fetomaternalen Grenzschicht

\section{A. Ostermeir' , C. Poets' , B. Spring ' , C. Gille' , N. Köstlin}

'Eberhard-Karls-Universität Universitätsklinik für Kinderheilkunde und Jugendmedizin, Neonatologie, Tübingen, Deutschland

Hintergrund. Frühgeburtlichkeit ist die Hauptursache für perinatale Morbidität und Mortalität. Immunreaktionen an der fetomaternalen Grenzschicht sind dabei eine mögliche Ursache. MDSC sind CD33-positive, myeloide Vorläuferzellen, die im Blut von Schwangeren, im Nabelschnurblut und in der Plazenta in erhöhter Zahl vorkommen und T-Zellantworten effektiv hemmen. Wie es zu dieser Akkumulation von MDSC in Schwangerschaft und Fetalzeit kommt ist bisher noch unklar. Hypothese. Die schwangerschaftsassoziierten Hormone Progesteron, Östrogen und humanes Choriongonadotropin (HCG) führen zur Akkumulation von MDSC und steigern ihre T-Zell-suppressive Aktivität.

Methoden. Zur Induktion von MDSC wurden periphere mononukleäre Zellen (PBMC) aus dem Blut von Erwachsenen isoliert, über sieben Tage mit unterschiedlichen Konzentrationen von Progesteron $(0,05-5 \mu \mathrm{g} / \mathrm{ml})$, Östrogen $(0,02-2 \mu \mathrm{g} / \mathrm{ml})$ und HCG $(0,05-5 \mathrm{IE} / \mathrm{ml})$ kultiviert und der Anteil an $\mathrm{CD}_{33}+$ Zellen durchflusszytometrisch bestimmt. Als Positivkontrolle diente die Zugabe von GM-CSF (1 ng/ml). Zur Bestimmung der T-Zell-Suppression wurden MDSC mittels magnetic activated cell sorting aus der Plazenta isoliert und im Verhältnis 1:2 zu CFSE-markierten und mit IL-2 und OKT3 stimulierten PBMC gegeben. Die Hormone Progesteron, Östrogen und HCG wurden in den oben genannten Konzentrationen hinzugegeben. Als Kontrolle dienten PBMC, denen nur die Hormone, nicht aber MDSC zugegeben wurden. Nach 96 Stunden wurde die T-Zellproliferation durchflusszytometrisch ermittelt.

Ergebnisse. Die Zugabe von Progesteron, Östrogen und HCG führte nicht zu einer Zunahme des Anteils an $\mathrm{CD}_{33}+$ Zellen in PBMC verglichen mit PBMC ohne Hormonzugabe. In hohen Konzentrationen $(5 \mu \mathrm{g} /$ $\mathrm{ml})$ steigerte Progesteron die T-Zell-suppressive Wirkung von MDSC. Dieser Effekt war bei Östrogen und HCG nicht zu beobachten. Die Zugabe von Progesteron, Östrogen und HCG zu PBMC ohne zugegebene MDSC beeinflusste die T-Zell-Proliferation nicht.

Schlussfolgerung. Weder Progesteron, noch Östrogen oder HCG alleine scheinen MDSC induzieren zu können. Progesteron, nicht aber Östrogen oder HCG kann in hohen Konzentrationen die T-Zell-suppressive Wirkung von MDSC steigern. Vermutlich sind weitere Faktoren bzw. ein Zusammenwirken verschiedener Faktoren für die Akkumulation von MDSC in der Schwangerschaft verantwortlich.

\section{P03-03}

Die verstärkte Induktion von Fox-P3 in T-Zellen Neugeborener ist abhängig vom Polarisationszustand infizierter Makrophagen

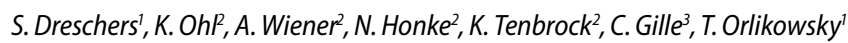
'Universitätsklinik für Kinder- und Jugendmedizin, Sektion für Neonatologie, Aachen, Deutschland, ${ }^{2}$ Kinder- und Jugendmedizin, Grundlagenforschung AG PD Dr. Tenbrock, Aachen, Deutschland, ${ }^{3}$ Eberhard-KarlsUniversität Universitätsklinik für Kin derheilkunde und Jugendmedizin, Neonatologie, Tübingen, Deutschland

Hintergrund. Sind Bakterien durch Phagozytose eliminiert, schließt sich eine Phase an, die die Entzündungsreaktionen beendet. Dieser Übergang ist kritisch und kann bei Frühgeborenen zur verlängerten Inflammationsreaktion („sustained inflammation“) führen. Die Polarisierung von Makrophagen in pro- und anti-inflammatorische Subtypen (M1, M2) hat einen Einfluss auf die funktionelle Reifung von T-Zellen: Regulatorische T-Zellen (T Regs) unterdrücken entzündliche Reaktionen und werden durch das Gleichgewicht von B7-Molekülen (u. a. CD8o, CD86) auf Makrophagen beeinflusst: Hohe Expression von CD86 führt zur Aktivierung von T Regs, d.h. Effektor T Zellen werden hinsichtlich Proliferation und Zytokinsekretion supprimiert. Hohe Expression von CD8o führt zur Suppression von T Regs mit Effektor T Zellaktivierung.
Makrophagen Neugeborener sind im Vergleich zu Makrophagen Erwachsenen in-vitro eingeschränkt in M1 und M2 polarisierbar.

Hypothese. Die Phagozytose von Bakterien durch polarisierte Makrophagen von Erwachsenen führt zu einer geringeren Induktion von Fox$\mathrm{P}_{3}+\mathrm{CD}_{4}+$ Lymphozyten (T Regs) als bei Neugeborenen.

Material und Methoden. Monozyten von Erwachsenen und aus Nabelschnurblut wurden durch M-CSF und IL- 4 bzw. IFN- $\gamma$ für 5 Tage polarisiert (PBMphi und CBMphi) und mit GFP-E. coli infiziert. Ermittlung der Zahl von Fox-P $3+C D 4+$ T Zellen sowie CD8o und CD 86 auf Makrophagen.

Ergebnisse. Nicht infizierte Proben (M1 und M2) enthielten 10,3 $\pm 2,7 \%$ Fox- $\mathrm{P}_{3}+\mathrm{CD}_{4}+\mathrm{T}$-Zellen in Nabelschnurblut und $15,7 \pm 0,1 \%$ im Blut von Erwachsenen. Nach Infektion stieg der prozentuale Anteil Fox-

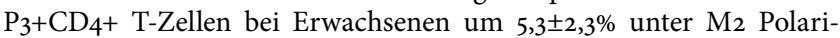

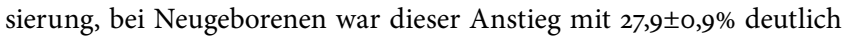
höher $(\mathrm{p}<0,05)$. Beide Gruppen zeigten keinen Anstieg unter Mi Polarisierung. CD8o wurde nach Infektion von CBMphi signifikant höher exprimiert als von PBMphi (3121,2 \pm 64 MFI vs. 1354,5 $\pm 288,7$ MFI), wobei die Hoch-Regulation von CD 86 sich nicht signifikant unterschied.

Schlussfolgerung. Unter M2-Polarisierung ist die Anzahl an induzierten Fox- $\mathrm{P}_{3}+\mathrm{CD}_{4}+\mathrm{T}-\mathrm{Zellen}$ bei Neugeborenen größer als beim Erwachsenen. Bei M2-polarisierten infizierten Makrophagen von Neugeborenen ist das Verhältnis der B7 Moleküle zur Expression von CD8o, eines reprimierenden Moleküls, verschoben. Durch die Hemmung von $\mathrm{T}$ Regs ist damit eine verstärkte Effektor T-Zellantwort im Sinne einer Inflammation möglich.

\section{P03-04}

Monozyten bleiben wachsam: periphagozytäre Reaktionen nach einer zweiten Infektion

\section{S. Dreschers', J. Kerth', C. Platen', C. Gille', T. Orlikowsky'}

'Universitätsklinik für Kinder- und Jugendmedizin, Sektion für Neonatologie, Aachen, Deutschland, ${ }^{2}$ Eberhard-Karls-Universität Universitätsklinik für Kinderheilkunde und Jugendmedizin, Neonatologie, Tübingen, Deutschland

Hintergrund. In vitro wird nach einer bakteriellen Infektion Phagozytose-induzierter Zelltod (PICD) von Monozyten ausgelöst, der beim Erwachsenen deutlicher ausgeprägt ist als beim Neugeborenen. Überlebende Monozyten können pro-inflammatorische Zytokine sezernieren und somit inflammatorische Vorgänge in Gang halten („sustained Inflammation").

Hypothese. Kommt es zu einer länger anhaltenden Infektion oder kurzfristigen Re-Infektion, wird eine zweite Phagozytose und die Produktion von pro- bzw. anti-inflammatorischen Zytokinen (TNF-alpha, IL-10) und reaktiven Sauerstoffspezies (ROS) vermindert. Bei Erwachsenen tritt diese Verminderung deutlicher auf als bei Neugeborenen.

Material und Methoden. In-vitro-Infektion mit unterschiedlich fluoreszierenden E. coli (GFP, ds-RED, Nil-Blau) von Monozyten aus Nabelschnurblut (CBMO) und Blut von Erwachsenen (PBMO) im 24 Stunden Intervall (do: Erst-Infektion; d1: Re-Infektion; MOI (multiplicity of infection=10). 30 Stunden nach Erst-Infektion Analyse von ROS-Produktion, phagozytären Indices, Apoptose-Induktion mittels FACS Analyse. Zytokinsekretion (TNF- $\alpha$, IL-10) wurde mittels ELISA quantifiziert. Ergebnisse. Die Re-Infektion führte bei PBMO und CBMO zu identischen Phagozytose-Indices wie bei Erstinfektion, jedoch war der Prozentsatz an PBMO, welche E. coli doppelt phagozytierten größer als bei CBMO $(\mathrm{p}<0,05)$ und der Anteil an „naiven“ CBMO, welche bei der Erst-Infektion nicht phagozytiert hatten, konsekutiv höher $(\mathrm{p}<0,05)$. Die ROS Produktion war bei PBMO und CBMO in der Re-Infektion im Vergleich zur Erst-Infektion nicht verringert. Nach Re-Infektion war die IL-10-Sekretion bei CBMO geringer ausgeprägt als bei PBMO $(\mathrm{p}<0,05)$. Nach der Erst-Infektion wurde TNF-avon CBMO geringer sezerniert als von PBMO $(\mathrm{p}<0,05)$. Nach Re-Infektion fand sich dieser Unterschied jedoch nicht mehr, d.h. sezernierte TNF- $\alpha$-Konzentrationen von PBMO und CBMO waren identisch. PICD war bei Erstinfek- 
tion bei PBMO deutlicher ausgeprägt als bei CBMO; bei Re-Infektion fanden wir keine signifikanten Unterschiede, sowohl im phagozytierenden als auch nichtphagozytierenden (Bystander-)Anteil.

Schlussfolgerung. PBMO und CBMO sind dazu in der Lage, bei einer Re-Infektion identische Prozentsätze an Bakterien zu phagozytieren, jedoch ist der Anteil an Monozyten, welche zweimal phagozytieren, bei PBMO höher. Während PBMO bei Erst-Infektion deutlich höhere Konzentrationen an TNF- $\alpha$ im Vergleich zu CBMO sezernieren, zeigt sich bei früher Re-Infektion von CBMO eine identische TNF- $\alpha$-Konzentration und reduzierte IL-10-Sekretion, die eine proinflammatorische Immunantwort induzieren. Dieser Umstand kann bei anhaltender Infektion, bzw. früher Re-Infektion von Frühgeborenen hinsichtlich inflammatorischer Organschäden von Bedeutung sein.

\section{P03-05 \\ Lactoferrin attenuates the early inflammatory response of neo- natal macrophages}

L. Wisgrill', E. Herndl', K. Sadeghi', A. Spittler', H. Helmer', P. Husslein ${ }^{3}$, E. Förster-Waldl', A. Berger'

${ }^{1}$ Medical University of Vienna, Department of Pediatrics and Adolescent Medicine, Division of Neonatology, Pediatric Intensive Care and Neuropediatrics, Vienna, Österreich, ${ }^{2}$ Medical University of Vienna, Department of Surgery and Core Facility Flow Cytometry, Vienna, Österreich, ${ }^{3}$ Department of Obstetrics and Gynecology, Vienna, Österreich

Introduction. Necrotizing enterocolitis, an idiopathic inflammatory bowel necrosis, is a common gastrointestinal emergency in very low birth weight premature neonates. Neonatal intestinal macrophages progressively acquire a gestational age- dependent non-inflammatory profile. Thus, the higher proinflammatory activity of premature intestinal macrophages might contribute to inflammatory mucosal injury leading to NEC. Lactoferrin, a mammalian milk glycoprotein, has various effects on the host innate immune defense and showed promising results in clinical studies to prevent late-onset sepsis and NEC in premature infants. Therefore, the aim of the study was to investigate the effect of human lactoferrin on LPS and LTA stimulated cord blood monocyte-derived macrophages of term and preterm neonates compared to healthy adults. Methods. Cord blood and peripheral blood monocytes were differentiated in the presence or absence of Lactoferrin and then stimulated with the TLR2 agonist LTA or the TLR4 agonist LPS. The surface expression of TLR2/TLR4, TLR- signaling, intracellular TNFa production and secretion were evaluated by flow cytometry.

Results. Lactoferrin attenuates in a dose-dependent manner the TNFa production of monocyte-derived macrophages among the age groups. This effect is mediated by a decreased TLR- expression of lactoferrin treated macrophages and resulted in diminished TLR-signaling of $\mathrm{p}_{3} 8$ and ERK $1 / 2$ upon stimulation with LPS and LTA.

Conclusion. In summary, human lactoferrin attenuates the proinflammatory response upon TLR2/TLR4 activation of neonatal macrophages. These data show that lactoferrin mediates anti-inflammatory effects by down-regulation of TLR 2 and TLR 4 expression on neonatal macrophages. We thus conclude that these anti-inflammatory properties might be a potential mechanism contributing to the preventive effects of lactoferrin in the premature gut.

\section{P03-06 \\ MDSC aus dem Nabelschnurblut polarisieren T-Zellen in Rich- tung TH2-Antworten und hemmen die Reifung von Monozyten}

N. Köstlin' ', B. Spring', A. Leiber', T. Orlikowsky'2, C. Poets', C. Gille'

'Eberhard-Karls-Universität Universitätsklinik für Kinderheilkunde und Jugendmedizin, Neonatologie, Tübingen, Deutschland, ${ }^{2}$ Universitätsklinik für Kinder- und Jugendmedizin, Sektion für Neonatologie, Aachen, Deutschland

Hintergrund. Neonatale Infektionen sind die wichtigste Ursache für perinatale Morbidität und Mortalität. Das Immunsystem des Neuge- borenen zeigt im Unterschied zum Immunsystem des Erwachsenen eine verminderte Fähigkeit zur Infektabwehr. Die Ursachen hierfür sind noch weitgehend unverstanden. Die neonatale T-Zell-Antwort ist charakterisiert durch das Überwiegen einer $\mathrm{TH}_{2}$-Antwort und einer verminderten TH1-Antwort. Außerdem scheint die costimulatorische Aktivität von Antigen-präsentierenden Zellen wie Monozyten beim Neonaten vermindert zu sein. Myeloide Suppressorzellen (MDSC) sind myeloide Vorläuferzellen, welche Funktionen anderer Immunzellen hemmen und im Nabelschnurblut gesunder Neugeborener in erhöhter Zahl vorkommen.

Hypothese. Nabelschnurblut-MDSC (NSB-MDSC) führen zu einer TZell-Polarisierung in Richtung $\mathrm{TH}_{2}$-Antworten und verändern die costimulatorische Aktivität von Monozyten.

Methoden. NSB-MDSC wurden aus Nabelschnurblut mittels „magnetic activated cell sorting" angereichert und im Verhältnis 1:2 zu mononukleären Zellen (PBMC) aus dem Blut gesunder Erwachsener gegeben. Nach fünf Tagen in Zellkultur erfolgte eine Oberflächenfärbung für CD4, CCR4, CCR6, CXCR3, CD14, HLA-DR, CD8o und CD86, sowie eine Intrazellulärfärbung für Interleukin 4 (IL-4) und Interferon? (IFN?). Als Kontrolle dienten PBMC ohne Zugabe von MDSC und PBMC, welche mit reifen Granulozyten kultiviert wurden.

Ergebnisse. Der Anteil an CD4+/CXCR3-/CCR4+/CCR6- TH2-Zellen war nach fünftägiger Co-Kultur von PBMC mit NSB-MDSC im Vergleich zur Kultur von PBMC alleine signifikant erhöht, während der

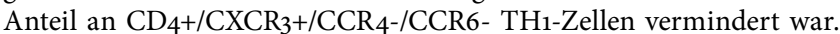
Ebenso war der Anteil an IL-4-produzierenden T-Zellen erhöht und der Anteil an IFN?-produzierenden T-Zellen vermindert. Auf Monozyten zeigte sich nach fünftägiger Co-Kultur mit NSB-MDSC eine verminderte Expression der co-stimulatorischen Moleküle HLA-DR und CD8o, während die Expression von CD86 unverändert blieb. Die Zugabe von reifen Granulozyten zu PBMC zeigte diese Effekte nicht.

Schlussfolgerung. NSB-MDSC bewirken eine Polarisierung von T-Zellen in Richtung $\mathrm{TH}_{2}$-Antworten und verringern die Expression costimulatorischer Moleküle auf Monozyten. Diese Ergebnisse deuten darauf hin, dass NSB-MDSC eine Bedeutung für die Immunregulation des Neugeborenen haben. Eine Beeinflussung der MDSC-Funktion könnte eine Möglichkeit sein, neue Ansätze in der Behandlung neonataler Infektionen zu entwickeln.

\section{P03-07}

\section{Hypoxien, Bradykardien und Impfantwort bei Frühgeborenen nach 6+1-fach-Impfung}

S. Vogel', A. Leiber', C. Gille', A. Bialkowski', K. Büchner', C. Poets'

'Eberhard-Karls-Universität Universitätsklinik für Kinderheilkunde und Jugendmedizin, Neonatologie, Tübingen, Deutschland

Hintergrund. Hypoxien und Bradykardien sind typische Begleitsymptome im Rahmen der Grund-Immunisierung Frühgeborener, was dazu führte, dass bei Frühgeborenen (FG) $<29$ Wochen die 1. Impfung nur noch unter stationären Bedingungen empfohlen wird. Daten von Erwachsenen zeigten, dass Impfreaktion und -erfolg tageszeitlichen Einflüssen unterliegen. Als Ursache wird die zirkardiane Regulation des Immunsystems angenommen.

Fragestellung. Untersucht werden sollte, ob es bei ehemals sehr unreifen FG Unterschiede in der Hypoxie-/Bradykardiehäufigkeit nach Impfung morgens vs. abends gibt.

Material und Methoden. In die Pilotstudie wurden zehn Frühgeborene zwischen $26+0$ und 30+6 Schwangerschaftswochen eingeschlossen. Davon wurden 4 morgens und 6 abends geimpft. Die Randomisierung erfolgte mittels verschlossener Umschläge, welche am Tag vor der Impfung gezogen wurden. Rekrutiert wurde aus dem Patientenkollektiv der Neonatologie der Universitäts-Kinderklinik Tübingen. Zur Detektion von Hypoxien und Bradykardien wurden im Zeitraum 24 Stunden vor bis 48 Stunden nach Impfung Pulsoxymetrie-Daten aufgezeichnet. Zudem wurde ein Schlaftagebuch geführt. Ausgewertet wurden nur Hypoxien und Bradykardien während des Schlafs. 24 Stunden 
nach Impfung wurden Interleukin-6 (IL-6) und C-reaktives Protein (CrP) als Marker für eine Immunreaktion bestimmt. Ausgeschlossen wurden schwer beeinträchtigte Kinder (BPD, PVL, IVH >2, schwere Fehlbildungen), sowie Kinder HBV-positiver Mütter. Zur Kontrolle des Impferfolges wurden vor Impfung sowie beim Kontrolltermin in der neonatologischen Ambulanz im korrigierten Alter von vier Monaten Impftiter für Pertussis und Haemophilus influenzae B bestimmt.

Ergebnisse. Hypoxien und Bradykardien traten in den ersten 24 Stunden nach abendlicher Impfung signifikant seltener auf als nach morgendlicher Impfung $(5,5 \pm 6,2$ vs. 19 $\pm 7,2$ Ereignisse; $p<0,05)$ bei ähnlichem Ausgangsniveau. Nahezu alle Frühgeborenen zeigten nach Impfung tageszeitunabhängig einen Temperaturanstieg sowie einen Anstieg der laborchemischen Entzündungsparameter IL-6 und CrP. Im korrigierten Alter von 4 Monaten zeigten 5 von 10 Kindern einen ausreichenden Impfschutz für Bordetellapertussis (FHA IgG $>38 \mathrm{IU} / \mathrm{ml}$ ). Alle hatten ihre erste Impfung abends erhalten. Ein Impfschutz gegen Haemophilus influenzae Typ B war in 3 von 10 Fällen vorhanden (IgG $>1 \mu g$ / $\mathrm{ml}$ ), auch hier war die Impfung immer abends erfolgt.

Schlussfolgerung. Hypoxie- und Bradykardiehäufigkeit nach Impfung sowie der Impferfolg scheinen vom Tageszeitpunkt der Impfung beeinflusst zu sein. Eine abendliche Impfung scheint bei besserem Impferfolg weniger Hypoxien und Bradykardien zu verursachen. Derzeit wiederholen wir die Untersuchung, um zu prüfen, ob sich diese überraschend eindeutigen Ergebnisse bestätigen lassen.

\section{P03-08 \\ Hypoxie führt zu einer erhöhten Expression von DMBT1 bei kultivierten Lungenepithelzellen sowie im Lungengewebe von Früh- und Neugeborenen}

\section{H. Müller', M. Renner', U. Felderhoff-Müser ${ }^{3}$, J. Mollenhauer ${ }^{4}$}

'Universitätsklinikum Essen, Klinik für Kinderheilkunde I/Perinatalzentrum, Essen, Deutschland, ${ }^{2}$ Institut für Pathologie, Universitätsklinikum Heidelberg, Heidelberg, Deutschland, ${ }^{3}$ Universitätsklinikum Essen, Klinik für Kinderheilkunde I/Perinatalzentrum, Essen, Deutschland, ${ }^{4}$ Lundbeckfonden Center of Excellence NanoCAN and Molecular Oncology, University of Southern, Odense, Dänemark

Hintergrund. Das Protein Deleted in Malignant Brain Tumor 1 (DMBT1) wird in der Lunge von Früh- und Neugeborenen sowie von Erwachsenen exprimiert. Bei Frühgeborenen steigt die Expression mit zunehmendem Gestationsalter. Bei allen Altersgruppen ist im Rahmen von pulmonalen Infektionen eine erhöhte DMBT1-Expression im Lungengewebe zu finden. Dabei kann DMBT1 zu einer Inaktivierung von Surfactant führen.

Fragestellung. Es wurde untersucht, ob Hypoxie einen Einfluss auf die Expression von DMBT1 in der Lunge hat.

Material und Methoden. Es wurde die Expression von DMBTi bei kultivierten Lungenepithelzellen (A549-Zellen) unter hypoxischen und normoxischen Bedingungen mittels RT-PCR untersucht. Im Lungengewebe von totgeborenen Kindern nach ausgeprägter fetaler Hypoxie (chronische Plazentainsuffizienz mit multiplen Infarkten, Nabelschnurtorsionen; jeweils kein Amnioninfektionssyndrom) wurde die DMBT1-Expression im Lungengewebe mittels Immunhistochemie detektiert. Um potentielle Bindungsstellen im DMBT1-Promotor zu finden, wurde das Programm Genomatix (www.genomatix.de) verwendet. Ergebnisse. DMBT1 wird im Rahmen einer Hypoxie $\left(1 \% \mathrm{O}_{2}, 5 \% \mathrm{CO}_{2}\right.$, 94\% N2) bei den kultivierten Lungenepithelzellen im Vergleich zu normoxischen Bedingungen hochreguliert. Bei nach fetaler Hypoxie totgeborenen Kindern ist die DMBT1-Expression stark erhöht. Mithilfe des Programms Genomatix ließ sich im Promotor von DMBT1 eine Bindungsstelle für HIF-1 finden. Dies lässt vermuten, dass HIF-1 bei der gesteigerten DMBT1-Expression unter Hypoxie eine wesentliche Rolle spielen könnte.

Schlussfolgerung. DMBT1 wird unter hypoxischen Bedingungen im Lungengewebe vermehrt exprimiert. Dabei könnte die Bindungsstelle für HIF-1 im DMBT1-Promotor wichtig sein.

\section{P03-09}

\section{Erkrankungen des Darmtraktes führen zu einer gesteigerten} DMBT1-Expression bei Früh- und Neugeborenen

H. Müller', M. Renner', B. Helmke', J. Mollenhauer', U. Felderhoff-Müser ${ }^{5}$ 'Universitätsklinikum Essen, Klinik für Kinderheilkunde I/Perinatalzentrum, Essen, Deutschland, ${ }^{2}$ Institut für Pathologie, Universitätsklinikum Heidelberg, Heidelberg, Deutschland, ${ }^{3}$ Institut für Pathologie, Elbe Kliniken Stade-Buxtehude, Stade, Deutschland, ${ }^{4}$ Lundbeckfonden Center of Excellence NanoCAN and Molecular Oncology, University of Southern, Odense, Dänemark, ${ }^{5}$ Universitätsklinikum Essen, Klinik für Kinderheilkunde I/Perinatalzentrum, Essen, Deutschland

Hintergrund. Das Protein Deleted in Malignant Brain Tumor 1 (DMBT1) hat Funktionen bei der angeborenen Immunabwehr und der epithelialen Differenzierung. Bei Erwachsenen konnte eine erhöhte Expression sowie eine wesentliche Funktion von DMBT1 bei chronisch entzündlichen Darmerkrankungen nachgewiesen werden.

Fragestellung. Es wurde die Expression von DMBT1 im Darmgewebe von Früh- und Neugeborenen mit nekrotisierender Enterokolitis, intestinaler Perforation und Volvulus untersucht und mit der intestinalen DMBT1-Expression von Feten, die ein vergleichbares Gestationsalter aufwiesen, verglichen.

Material und Methoden. Untersucht wurde die DMBT1-Expression in fetalen Geweben unterschiedlichen Gestationsalters, die von der Firma Biocat käuflich erworben wurden, sowie in den Geweben von Totgeburten und verstorbenen Früh- und Neugeborenen und in bei Operationen entfernten Darmgeweben, die durch das Pathologische Institut der Universität Heidelberg untersucht wurden. DMBT1 wurde in den Geweben mittels Immunhistochemie und RNA-in-situ-Hybridisierung detektiert.

Ergebnisse. DMBT1 wird im Darm des humanen Feten sehr früh exprimiert und ist bereits in der 14. Schwangerschaftswoche nachweisbar. Im Rahmen einer nekrotiserenden Enterokolitis, einer intestinalen Perforation oder eines Volvulus kommt es zu einer Induktion der DMBT1Expression, die deutlich über der intestinalen DMBT1-Expression des jeweiligen Gestationsalters liegt.

Schlussfolgerung. DMBT1 wird schon früh im sich entwickelnden Darmtrakt des humanen Feten exprimiert und bei neonatalen Erkrankungen des Darms, die mit einer Entzündung assoziiert sind, hochreguliert. DMBT1 scheint deshalb auch bei Neu- und Frühgeborenen eine Funktion bei der lokalen angeborenen Immunabwehr des Darms zu haben.

\section{Posterwalk 4 - Seltene Erkrankungen 1}

\section{P04-01}

Schwere arterielle Hypotonie als klinisches Symptom einer genetisch oder medikamentös bedingten renal tubulären Dysgenesie

J. Pansy', G. Pichler', H. Hubmann'2, E. Karpf', C. Bergmann 4 , C. Mache², B. Acham-Roschitz' ${ }^{2}$ E. Ring ${ }^{2}$

${ }^{1}$ Universitäts Klinik für Kinder- und Jugendheilkunde Graz, Medizinische Universität Graz, Klinische Abteilung für Neonatologie, Graz, Österreich, ${ }^{2}$ Universitätsklinik für Kinder-und Jugendheilkunde Graz, Medizinische Universität Graz, Klinische Abteilung für Allgemeinpädiatrie, Graz, Österreich, ${ }^{3}$ Institut für Pathologie, Medizinische Universität Graz, Graz, Österreich, ${ }^{4}$ Zentrum für Humangenetik, Bioscientia Klinik, Ingelheim, Deutschland

Hintergrund. Die renal tubuläre Dysgenesie (RTD) ist ein meistens letales autosomal-rezessives Krankheitsbild mit histologisch fehlender oder verminderter Ausbildung des proximalen Tubulus der Nieren. Ursächlich sind Mutationen im Renin-Angiotensin-Systems (RAS). Die histologisch idente fetal erworbene Form der RTD ist mit der Einnahme mütterlicher Medikamente (speziell ACE-Inhibitoren, AT-II-RezeptorAntagonisten), dem fetofetalen Transfusionssyndrom (Spenderzwilling 
betroffen), schweren kardialen Fehlbildungen oder schweren Lebererkrankungen (Hämochromatose) assoziiert. In allen Fällen kommt es zu einer Blockade des RAS mit renaler Minderperfusion und folgendem klinischen Bild: fetales Oligo- oder Anhydramnion bei sonographisch normal großen, vielfach echodichten Nieren, neonataler Niereninsuffizienz, schwere arterielle Hypotonie und Beatmungsprobleme infolge einer Lungenhypoplasie.

Fallberichte. Fallbericht 1 - erworbene RTD: Ein weiblicher Säugling wurde bei Oligohydramnion mittels Sectio in der 32. SSW geboren. Postnatal bestand eine Kreislaufhypotonie mit hohem Katecholaminbedarf. Sonographisch bestanden echodichte, normal große Nieren. Bei oligoanurischem Nierenversagen wurde am 5. Lebenstag eine Peritonealdialyse begonnen und nach 8 Tagen bei suffizienter Diurese beendet. Auch die Kreislaufunterstützung konnte beendet werden. Die bei Verdacht einer RTD durchgeführte genetische Untersuchung war unauffällig. Wiederholte Befragungen der Mutter ergaben schließlich die Einnahme von Candesartan in der 26.-27. SSW (16 mg/Tag, kumulative Dosis $250 \mathrm{mg}$ ). Bei der letzten ambulanten Kontrolle mit 48 Monaten zeigte sich das Mädchen normal entwickelt, mit sonographisch kleinen, echodichten Nieren, ohne kortikomedulläre Differenzierung, Kreatinin $0,54 \mathrm{mg} / \mathrm{dl}$, Harnstoff $23 \mathrm{mg} / \mathrm{dl}$, Cystatin C mit 1,24 mg/l erhöht. Fallbericht 2 - angeborene RTD: Ein Frühgeborenes der 33. SSW mit Anhydramnion bei normal großen, echodichten fetalen Nieren ohne Harnstauung wurde spontan geboren. Postnatal wurde das Frühgeborene bei IRDS III ${ }^{\circ}$ intubiert. Trotz Surfactantgabe musste die Beatmung auf Hochfrequenzoszillation und NO-Therapie umgestellt werden. Ein NO-Auslassversuch scheiterte, sodass von einem persistierendem pulmonalen Hypertonus und einer grenzwertigen Lungenhypoplasie ausgegangen werden musste. Weiters bestanden eine ausgeprägte arterielle Hypotonie (trotz mehrmaliger Volumengabe, drei Katecholaminen und Solucortef) und ein anurisches Nierenversagen (Serumkreatinin 2,72 mg/dl, Harnstoff $92 \mathrm{mg} / \mathrm{dl}$, Kalium 7,9 mmol/l). Die Patientin verstarb am 3. Lebenstag. Die genetische Untersuchung bei Verdacht einer RTD ergab eine neue homozygote Mutation im ACE-Gen (Chromosom 17q23; Exon 9, c.1487G>A (p.Arg496Gln).

Schlussfolgerung. Die Kombination eines renalen Oligo- oder Anhydramnions mit sonographisch normal großen, nicht gestauten Nieren und postnatale Niereninsuffizienz, therapierefraktäre Hypotonie und Lungenhypoplasie kann wegweisend für eine RTD sein. Die exakte Evaluation der mütterlichen Medikamenteneinnahme (insbesondere Antihypertensiva) ist neben der genetischen Evaluation wichtig. Jede medikamentöse Therapie in der Schwangerschaft muss gut überlegt werden, aber ACE-Inhibitoren oder AT-II-Rezeptorblocker sollten in der Schwangerschaft obsolet sein.

\section{P04-02 \\ Brachytelephalangic chondrodysplasia punctata with a new hemizygous missense mutation in a neonate. A rare cause for severe respiratory distress in a newborn}

\section{S. Meyer', M. Gencik2 ', C. Wollny³, B. Oehl-Jaschkowitz², L. Gortner ${ }^{3}$}

'Universitätsklinikum des Saarlandes, Klinik für Allgemeine Pädiatrie und Neonatologie, Homburg, Deutschland, ${ }^{2}$ Biomedizinisches Zentrum, Gemeinschaftspraxis für Humangenetik, Homburg/Saar, Deutschland, ${ }^{3}$ Unversitätsklinik für Kinder und Jugendmedizin Gebäude ${ }^{9}$, Homburg, Deutschland

Background. Chondrodysplasia punctata (CDP) is a heterogenous group of skeletal dysplasias. The hallmark of CDP is aberrant bone mineralization, manifesting radiologically as epiphyseal stippling. Among this group of skeletal dysplasia, brachytelephalangic CDP (BCDP) is probably under-reported. Here, we report on a neonate with BCDP with a new hemizygous missense mutation (c.332G $>$ A p.Arg11His in Exon 5 of the ARSE-gene) and severe spinal compression leading to quadriplegia. Results. A 3,080-g infant boy was born at 372 weeks' gestation to a gravida III, para II mother by spontaneous vaginal delivery (unrelated parents). The pregnancy was uneventful except for polyhydramnios and absent fetal hiccups, but normal fetal movements. The neonate had a moderately depressed midface with a hypoplastic nose with barely patent nares. The child was initially flaccid (APGAR scores of 2 and 5 at 5 and 10 minutes) with absent spontaneous movements. On chest X-ray pulmonary hypoplasia and bilateral pneumothoraces were seen. Later in the course, slightly improved motor activity of both upper and lower extremities was noted. A skeletal survey demonstrated changes with multiple punctate calcifications of the epiphyses and vertebrae. The infant remained respirator-dependent. On ultrasonography, paresis of the diaphragm was demonstrated. On cerebral MRI and 3-dimensional reconstruction, severe compression of the cervical spine and subluxation of $\mathrm{C}_{1}$ and $\mathrm{C}_{2}$ was noted. On day 27 of life, spinal decompression just below the foramen magnum and stabilization of $\mathrm{C}_{1}$ and $\mathrm{C} 2$ was performed. Following surgery, the infant remained ventilator-dependent and a tracheostomy was performed. The infant is alive on a home-respirator without deterioration of neurologic symptoms. In our patient, a new hemizygous missense mutation c.332 $\mathrm{G}>\mathrm{A}$ (p.Arg11His) could be identified in Exon 5 of the ARSE-gene.

Conclusions. It is important to take into consideration rare causes for respiratory failure. In children with $\mathrm{BCDP}$, respiratory failure may be due to severe cervical spinal compression.

\section{P04-03}

Vena-galeni-Malformation - ungewöhnliche Ursache eines Hydrops fetalis bei einem Frühgeborenen

\section{S. Baranowski', J. Essers', B. Schmitz², H. Hummler'}

${ }^{1} K$ linik für Kinder- und Jugendmedizin, Universitätsklinikum Ulm, Sektion Neonatologie und Pädiatrische Intensivmedizin, Ulm, Deutschland, ${ }^{2}$ Klinik für Diagnostische und Interventionelle Radiologie, Sektion Neuroradiologie, Universitätsklinikum Ulm, Günzburg, Deutschland

Hintergrund. Die Differentialdiagnose des Hydrops fetalis ist vielfältig. Neben immunologischen kommen auch nichtimmunologische Ursachen wie Infektionen oder kardiale Ursachen unterschiedlicher Genese in Betracht.

Fallbericht. Bis 28 SSW unauffällige Vorsorge, mit $32+1$ SSW erstmals sonographisch Aszites und Pleuraergüsse, pathologisches CTG, pathologischer Doppler („reverse flow“), daher Indikation zur Sectio. Entbindung eines männlichen Frühgeborenen mit ausgeprägten generalisierten Ödemen, APGAR 3/5/6, Intubation im Kreissaal, Stabilisierung erst mittels HFO-Beatmung. Bei Hydrops fetalis Durchführung einer Echokardiographie mit Nachweis eines sehr dilatierten rechten Ventrikels und Zeichen einer relevanten pulmonalen Hypertonie. Schädelsonographisch Nachweis einer großen Vena galeni Malformation mit Hydrocephalus internus und periventrikulärer Zystenbildung bds. Im Verlauf Multiorganversagen. Eine neuroradiologische Intervention bei sehr instabilem kardiorespiratorischem Status (Bicêtre-Score von 4) erschien nicht sinnvoll. Es erfolgten ausführliche Gespräche mit den Eltern. Bei zunehmender Kreislaufinsuffizienz (MAD 18-19 mmHg) und progredienter Laktatazidose (zuletzt $29 \mathrm{mmol} / \mathrm{l}$ ) wurde die Intensivtherapie beendet.

Diskussion. Die Vena-galeni-Malformation ist die häufigste vaskuläre Fehlbildung des Gehirns bei Neugeborenen. Häufig wird diese AVMalformation schon bei den sonografischen Screening-Untersuchungen in der Schwangerschaftsvorsorge diagnostiziert. Neugeborene mit großen AV-Malformationen fallen häufig postpartal durch eine nicht beeinflussbare Herzinsuffizienz und/oder Flow-bedingter pulmonaler Hypertonie auf, die durch den ausgeprägten Links-Rechts-Shunt über die AV-Malformation zustande kommt. Dies kann bis zum Vollbild eines Hydrops fetalis führen, was bisher nur in wenigen Einzelfallberichten beschrieben ist. Die interventionelle Therapie (Embolisation) ist mit erheblichen Risiken assoziiert. Die Indikation kann anhand des „Bicêtre neonatal evaluation Scores" abgeschätzt werden. Dabei werden neben der kardialen $u$. a. auch die zerebrale und pulmonale Situation berücksichtigt. Bei einem Score $<8$ bestehen wie bei unserem Patienten keine sinnvollen Therapiemöglichkeiten. 
P04-04

\section{Das Long-QT-Syndrom, eine seltene aber lebensbedrohliche Ursache fetaler Arryhthmien - ein Fallbericht}

H. Schützle', R. Gebauer', A. Heilmann', J. Weichsel', R. Lachmann', S. Brenner' 'Universitätsklinikum Carl Gustav Carus Kinderklinik, Neonatologie, päd. Intensivmedizin, Dresden, Deutschland, ${ }^{2}$ Herzzentrum Leipzig, Kinderkardiologie/Rhythmologie, Leipzig, Deutschland, ${ }^{3}$ Universitätsklinikum Carl Gustav Carus Kinderklinik, Kinderkardiologie, Dresden, Deutschland, ${ }^{4}$ Universitätsklinikum Carl Gustav Carus Kinderklinik, Gynäkologie und Geburtshilfe, Dresden, Deutschland

Einleitung. Das Long-QT-Syndrom (LQTS) stellt eine Gruppe genetisch bedingter Störungen verschiedener Ionenkanäle des Herzmuskels dar, die mit einer Verlängerung des QT-Intervalls im EKG einhergehen. Dadurch wird das Auftreten tachykarder, oft selbstlimitierender Herzrhythmusstörungen (Torsades de Pointes) mit konsekutiver Synkope oder plötzlichem Herztod (ca. 10\% aller plötzlichen Säuglingstodfälle) begünstigt. Die Inzidenz des LQTS liegt bei ca. 1:2500 Lebendgeborenen. Pränatal fallen betroffene Patienten häufiger durch eine grenzwertig niedrige Herzfrequenz (110-120/min) und seltener durch Tachyarrhythmien oder Herzinsuffizienzzeichen auf.

Fallbericht. Bei unserem Patienten traten pränatal Tachyarrhythmien, insbesondere gehäufte Extrasystolen auf. Am Geburtstag zeigten sich sonographisch erstmals Zeichen einer fetalen kardialen Dekompensation in der Tachykardie. Dies war die Indikation zur primären Sectio mit 35+2 SSW. Postnatal bestand CPAP-Bedarf. Ein Pleura- oder Perikarderguss lagen nicht vor. Die Diurese war regelrecht, das Laktat lag im Normbereich. Auskultatorisch fiel eine Arrhythmie mit stark schwankenden Herzfrequenzen auf. Das EKG zeigte wiederholt auftretende selbstlimitierende ventrikuläre Tachykardien sowie Torsades de Pointes Salven bei einer QTc-Zeit >500 ms und pathologischer Repolarisationsmorphologie. In der invasiven Blutdruckmessung sowie echokardiographisch zeigte sich ein insuffizienter Auswurf in den Arrhythmiephasen ohne Anhalt für ein Vitium cordis. Unter EsmololDauerinfusion zeigte sich keine Besserung der tachykarden Episoden. Bei drohender kardialer Dekompensation erfolgte die Verlegung ins Herzzentrum Leipzig noch am 1. Lebenstag. Bei Verdacht auf ein LQTS Typ 3 erfolgte eine Bolusgabe Lidocain, gefolgt von einer Lidocaindauerinfusion. Dadurch konnte ein stabiler Sinusrhythmus etabliert werden. Vorübergehend wurde zur Stabilisierung des Rhythmus auch eine transösophageale kardiale Stimulation angewandt. Das Ansprechen auf die Lidocaintherapie unterstützte die Verdachtsdiagnose eines LongQT-3-Syndromes. Nach Stabilisierung der kardialen Funktion wurde die Therapie auf Propranolol und Mexiletin p.os. umgestellt, worunter keine ventrikulären Tachykardien mehr auftraten.

Ergebnisse. Soweit bislang beurteilbar ist die neurologische Entwicklung des Jungen unbeeinträchtigt. Beide Eltern haben ein normales Ruhe-EKG und sind klinisch asymptomatisch. Die molekulargenetische LQTS-Diagnostik ist ausstehend.

Schlussfolgerung. Der Fall zeigt, wie wichtig eine frühe Diagnosestellung und adäquate Therapie zur Vermeidung einer kardialen Dekompensation bis hin zum plötzlichen Herztod bei Patienten mit einem Long QT Syndrom ist. Vermutlich ist auch die Inzidenz des Long-QTSyndromes bei Kindern, die an einem plötzlichen Säuglingstod versterben höher als bislang vermutet.
P04-05

Body-stalk-Anomalie: Omphalozele, Thoraxhypoplasie, Blasenextrophie, Analatresie, Spinalrohrdefekt und Klumpfüße eines monochorioten, diamniotischen weiblichen Zwillings

S. Breindl', K. Gloning ${ }^{2}$, K. Haselwarter ${ }^{3}$, S. Seeliger ${ }^{4}$

${ }^{1}$ Kliniken St. Elisabeth, Klinik für Kinder und Jugendliche, Neuburg a.d. Donau, Deutschland, ${ }^{2}$ Pränatalmedizin München, München, Deutschland, ${ }^{3}$ Klinikum Ingolstadt, Klinik für Gynäkologie und Geburtshilfe, Ingolstadt, Deutschland, ${ }^{4}$ Kliniken St. Elisabeth, Klinik für Kinder und Jugendliche, Neuburg a.d. Donau, Deutschland

Einleitung. Die Body-stalk-Anomalie ist ein sporadisch auftretendes letales Fehlbildungssyndrom mit einer Inzidenz von ca. 1:14.00o Geburten. In der Literatur sind 6 Fälle bei monchorioter Zwillingsschwangerschaft beschrieben. Die Ursache wird in einer frühen embryonalen Entwicklungsstörung vermutet. Exogene Ursachen und genetische Veränderungen sind bis dato nicht beschrieben, so dass ein Wiederholungsrisiko fast vollständig ausgeschlossen werden kann. Auf Grund der Schwere der Fehlbildungen und der infausten Prognose verbietet es sich ethisch mit intensivmedizinischen Therapien zu beginnen.

Fallbericht. Bei vorzeitigem Blasensprung und ansteigenden Entzündungsparametern des führenden Zwillings mit präpartal beschriebener Body-stalk-Anomalie wird aus geburtshilflicher Sicht, um den zweiten gesunden Zwilling vor einer Infektion zu schützen, in der 30+4 Schwangerschaftswoche der Entschluss zur Sektio gestellt. Entbunden wird ein 1155 g schweres weibliches Frühgeborenes, Länge $32 \mathrm{~cm}$, Kopfumfang $26 \mathrm{~cm}$, APGAR 2/1/1, Ns-pH art. 7,38 mit Omphalocele, Blasenextrophie, Analatresie, schwerer lumbosakraler Kyphose, Meningomyelozele, Klumpfüßen beidseits, Arnold-Chiari-Malformation und Thoraxhypoplasie. Nach primärer palliativer Versorgung in warmen Tüchern wird das Kind den Eltern übergeben, wo es 45 Minuten später an der respiratorischen Insuffizienz verstirbt. Der zweite weibliche Zwilling ist gesund und benötigt in den ersten Lebenstagen zur Atemunterstützung CPAP-Atemhilfe.

Schlussfolgerung. Body-stalk-Anomalie, auch bekannt als Syndrom der kurzen Nabelschnur, ist ein sehr selten auftretendes Ereignis. Auf Grund der guten pränatalen Diagnostik kann dieses schwere Fehlbildungssyndrom früh erkannt werden, um die Eltern gut zu beraten und zu begleiten. Im Vorfeld sollte die infauste Prognose thematisiert werden, da Früh- und Neugeborene Atemanstrengungen aufweisen und auch über fast eine Stunde Zeichen des Lebens zeigen können.

\section{P04-06}

Kongenitaler FLIT („fetal lung interstitial tumor“) - eine neue Tumorentität als seltene Ursache einer schweren perinatalen Asphyxie

I. Pilz-Klement', S. Horsch', A. Höck', A. Retzlaff', M. Schönbeck', B. Fleige², E. Harps ${ }^{1}$

'Helios-Klinikum Berlin Buch, Neonatologie, Berlin Buch, Deutschland, ${ }^{2}$ Helios-Klinikum Berlin Buch, Institut für Gewebediagnostik Berlin, Berlin, Deutschland

Hintergrund. Angeborene Raumforderungen der Lunge sind selten. Differentialdiagnostisch kommen verschiedenste pulmonale Malformationen sowie seltene Neoplasien (z. B. pleuropulmonales Blastom) in Frage. Eine relativ neue Tumorentität stellt der FLIT („fetal lung interstitial tumor") dar. Bisher sind nur wenige Fälle beschrieben. Wir präsentieren hier den Fall eines kongenitalen FLIT, der pränatal unerkannt, aufgrund der Lungen- und Mediastinalverlagerung zu einer schweren perinatalen Asphyxie führte.

Falldarstellung. Das männliche Neugeborene wurde nach unauffälliger Schwangerschaft per Notsectio bei fetaler Bradykardie in einem peripheren Krankenhaus geboren. Postnatal entwickelte er eine schwere respiratorische Anpassungsstörung, Nabelschnur-pH 6,8; APGAR 3/3/2. Nach Reanimation und Stabilisierung wurde das Kind in kritischem 

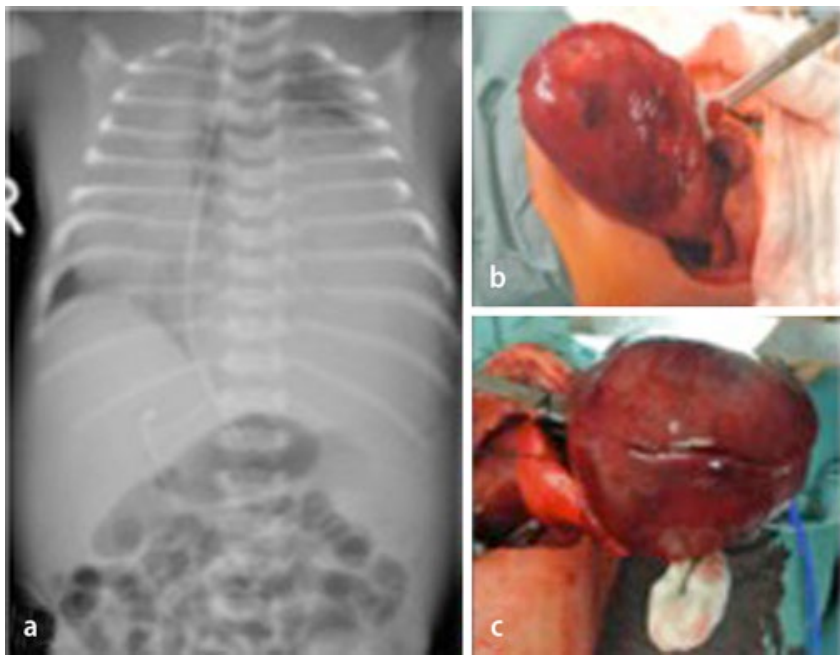

Abb. P04-06 $\triangle$

Zustand, intubiert in die Kinderklinik übernommen. Das Röntgenbild des Thorax zeigte eine massive Raumforderung, die den gesamten linken Thorax einnahm, zur Verdrängung von Mediastinum, Leber und Magen sowie zu Atelektasen der kontralateralen Lunge führte (Abb. Po4-06 a). Bei persistierender fetaler Zirkulation wurde iNO erfolgreich eingesetzt. Ein CT bestätigte den Befund einer abgegrenzten, teils zystisch imponierenden Raumforderung. Differentialdiagnostisch wurde an ein Neuroblastom oder pleuropulmonales Blastom gedacht. Am 2. Lebenstag erfolgte die vollständige operative Entfernung des makroskopisch scharf begrenzten Tumors (80×65×50 mm; Abb. Po4-06 b,c). Histopathologisch konnte ein FLIT nachgewiesen werden. Der postoperative Verlauf gestaltete sich durch eine peristierende pulmonale Hypertonie, die sich unter iNO, Inotropika und Hochfrequenzoszillation langsam besserte, und rezidivierende linksseitige Pneumothoraces, die insgesamt eine Drainage über 11 Tage notwendig machten, zunächst schwierig. Am 7. postoperativen Tag konnte der Patient extubiert werden und ab dem 13. postoperativen Tag benötigte er keine Atemhilfe mehr. Diskussion. FLIT stellt eine eigene Entität des pleuropulmonalen Blastoms dar und präsentiert sich charakteristischerweise als solider oder gemischt solider/zystischer Lungentumor. Er besteht aus unreifem Mesenchym und irregulären, alveolar-ähnlichen Strukturen die dem unreifen, kanalikulären Stadium der fetalen Lunge (20-24 SSW) ähneln. In den wenigen bisher beschriebenen Fällen traten nach vollständiger Entfernung des Tumors keine Lokalrezidive oder Metastasen auf, so dass wir bei unserem Patient von einer vollständigen Heilung ausgehen. Die Kenntnis dieses Krankheitsbildes und eine idealerweise pränatal gestellte Diagnose ermöglichen eine optimierte, interdisziplinäre Versorgung in einer Klinik mit Neonatologie und Kinderchirurgie.

\section{P04-07}

\section{Erster Fall einer Bronchitis fibroplastica bei Morbus Hodgkin}

\section{K. Alfen', C. Dohna-Schwake'}

\section{'Universitätsklinikum Essen, Kinderheilkunde ', Essen, Deutschland}

Einleitung. Die Bronchitis fiibroplastica ist eine seltene Erkrankung, bei der es zu einem Verschluss von Bronchien durch einen aus Schleim, Fibrinfäden, Epithel- und Entzündungszellen bestehenden zähen Pfropf kommt, der zur Atelektase des betroffenen Lungenabschnittes führt. Auslöser sind meist Atemwegserkrankungen wie Pneumonien, Asthma und Tuberkulose. Im Kindesalter ist sie vor allem als Komplikation nach operativer Korrektur komplexer Herzfehler, v. a. nach FontanOperation bekannt.

Fallbericht. Wir beschreiben eine 17-jährige Patientin, die sich mit seit zwei Wochen bestehendem Reizhusten und nächtlicher Dyspnoe vorstellte. Radiologisch zeigten sich ein Mediastinaltumor im mittleren Mediastinum sowie eine subtotale Atelektase der linken Lunge. Nach Lymphknotenbiopsie konnte histologisch die Diagnose eines klassischen Hodgkin-Lymphoms gestellt werden. Es wurde eine Polychemotherapie gemäß GPOH-HD-Register begonnen. Innerhalb weniger Tage verschlechterte sich die pulmonale Situation bis hin zur respiratorischen Insuffizienz und der Notwendigkeit der Beatmung. Bronchoskopisch zeigte sich eine komplette Verlegung des linken Hauptbronchus durch einen zähen, langstreckigen Sekretpfropf. Nach bronchoskopischer Entfernung konnte die Beatmung schrittweise deeskaliert werden. Es folgten mehrfache Rezidive mit der Notwendigkeit der bronchoskopischen Entfernung von neuen Sekretpfröpfen. Als Rezidiv-Prophylaxe begannen wir eine Inhalationstherapie mit Alteplase (rekombinanter gewebespezifischer Plasminogenaktivator, rt-PA). Darunter verflüssigten sich die zähen Pfröpfe, ließen sich leichter konventionell absaugen und bildeten sich schließlich nicht mehr neu. Eine erneute Bronchoskopie war nicht notwendig, und die Patientin konnte nach einer Woche problemlos extubiert werden.

Schlussfolgerung. Zusammenfassend handelt es sich um die Erstbeschreibung einer Bronchitis fibroplastica bei M. Hodgkin. Als Auslöser ist zu diskutieren, dass es durch die ungewöhnliche Lokalisation im mittleren Mediastinum zu einem Abpressen von Lymphflüssigkeit und gleichzeitig Verengung des linken Hauptbronchus gekommen ist. Die Inhalation mit Alteplase ist eine wirkungsvolle Therapie dieser seltenen Komplikation.

\section{P04-08}

\section{Morbus Sly - eine seltene Ursache für einen Hydrops fetalis. Fall-} beschreibung und Literaturreview

\section{Blaßfeld', S. Horsch'1, M. Schönbeck'1, A. Retzlaff', A. Höck', E. Harps²}

${ }^{1}$ Helios-Klinikum Berlin Buch, Neonatologie, Berlin, Deutschland, ${ }^{2}$ HeliosKlinikum Berlin Buch, Neonatologie, Berlin Buch, Deutschland

Hintergrund. Die Ursachen eines nichtimmunologischen fetalen Hydrops (NIHF) sind vielfältig und bleiben häufig ungeklärt. Eine seltene Diagnose ist die Mukopolysaccharidose Typ VII (Morbus Sly), eine lysosomale Speicherkrankheit (Inzidenz 1:250.0oo) verursacht durch eine $\beta$-Glucuronidase-Defizienz. Wir stellen hier den Fall eines Frühgeborenen mit NIHF vor, bei dem eine rasche Diagnose des M. Sly gelang. Fallvorstellung. Aufgrund eines zunehmenden Hydrops fetalis wurde das weibliche Frühgeborene bei $32+2$ SSW per Sectio cesarea geboren. Postnatal erfolgten simultan die primäre Intubation mit Surfactantgabe bei respiratorischer Insuffizienz, sowie die Anlage einer Aszitesund einer Pleuradrainage. Unter der Beatmung mit einem max. $\mathrm{FiO}_{2}$ von 0,4 und intravenöser Gabe von Volumenboli und FFP kam es zu einer raschen kardiorespiratorischen Stabilisierung. Die Extubation erfolgte bereits an Tag 3 mit anschließender Atemhilfe bis Tag 7. Die Pleura- und Aszitesdrainage konnten an Tag 4 und 5 entfernt werden. Bei einem Geburtsgewicht von $2830 \mathrm{~g}$ waren die Gewebsödeme unter Diuretikatherapie und Eiweißsubstitution rasch regredient mit einem Minimalgewicht von $1770 \mathrm{~g}$ (Tag 8). Eine voll orale Ernährung war ab Tag 9 möglich. Die Untersuchungen hinsichtlich einer infektiösen oder kardiovaskulären Genese, sowie die laborchemische Basisdiagnostik inklusive einer Chromosomen-Analyse und des erweiterten Stoffwechselscreenings blieben ohne pathologischen Befund. Die ergänzenden Sonographien ergaben keinen Anhalt für Fehlbildungen oder Tumore. Mit dem wegweisenden Befund einer Defizienz der $\beta$-Glucuronidase stellten wir schließlich an Tag 15. die Diagnose des M. Sly.

Diskussion. Der Morbus Sly ist eine seltene und vermutlich unterdiagnostizierte Erkrankung. Schwere neonatale Formen verlaufen letal, aber auch mildere Verläufe mit komplikationsarmer Neonatalperiode werden beschrieben. Die Prognoseeinschätzung bleibt bei geringen Fallzahlen und breitem phänotypischem Spektrum schwierig. Unsere Patientin konnte trotz des schweren Hydrops fetalis gut stabilisiert werden. Bei dieser Patientengruppe könnte es bei frühzeitiger Diagnose in naher Zukunft neue Therapieoptionen geben. In Tiermodellen werden 
derzeit retrovirale Gentherapien sowie Enzymersatztherapien (ERT) untersucht. Eine klinische Studie zur ERT mit rekombinanter humaner $\beta$-Glucuronidase läuft derzeit in Großbritannien. Erste Therapieversuche zeigten in den USA bereits deutliche klinische Besserungen. In Deutschland werden Therapieversuche in Einzelfällen diskutiert, aktuell wird neben der symptomatischen Therapie unter Risikoabwägung nur die Stammzelltransplantation bei frühzeitiger Diagnosestellung praktiziert.

\section{P04-09}

\section{Normale Fruchtwassermenge trotz Nierenagenesie?!}

S. Kunzmann', I. Klaassen², T. Diehl'², W. Diehl³, U. Gottschalk', J. Oh', H. Schäfer ${ }^{6}$, D. Singer ${ }^{2}$

'Universitäts-Kinderklinik, Würzburg, Deutschland, ${ }^{2} U n i v .-K i n d e r k l i n i k$ Eppendorf, Sektion Neonatologie/Päd. Intensivmedizin, Hamburg, Deutschland, ${ }^{3}$ Klinik für Geburtshilfe und Pränatalmedizin, UKE, Hamburg, Deutschland, ${ }^{4}$ Klinik für Kinderkardiologie, Universitäres Herzzentrum, Hamburg, Deutschland, ${ }^{5}$ Univ.-Kinderklinik Eppendorf, Hamburg, Deutschland, ${ }^{6}$ UKE, Institut für Pathologie, Hamburg, Deutschland

Hintergrund. Eine veränderte Fruchtwasser(FW)-Menge besitzt eine hohe perinatologische Relevanz. Die genaue Regulation der FW-Produktion und -Resorption ist bisher nicht hinreichend verstanden, weswegen eine intrauterine medikamentöse Beeinflussungsmöglichkeit der FW-Produktion derzeit nicht zur Verfügung steht.

Fallbericht. Hypotrophes männliches Frühgeborenes von $33+1$ SSW (GG 1870 g) mit einem komplexen Fehlbildungssyndrom. Pränatal bestand neben einem schweren Herzfehler (Ebstein-Anomalie) der V. a. auf eine Blasenekstrophie bei fehlender Darstellbarkeit der Harnblase. Zusätzlich V. a. auf eine intestinale Obstruktion, Darstellung einer Lippenspalte und Nachweis einer deutlichen Wachstumsretardierung. Während der gesamten Schwangerschaft konnte stets eine unauffällige Fruchtwassermenge nachgewiesen werden. Nach der Geburt (Apgar 4/7/8) bestand zunächst ein klinisches Kombinationsbild aus RDS und PPHN. Unter symptomatischer Therapie (Surfactant, HFOV, NO, Katecholamine) war eine kardiorespiratorische Stabilisierung möglich. Eine Blasenekstrophie bestätigte sich nicht. Im Verlauf jedoch Entwicklung einer schweren Niereninsuffizienz bei Anurie, sonographisch (ebenso wie post mortem) kein Nachweis von Nierengewebe, Ureteren oder einer Harnblase. Zusätzlich Bestätigung einer Dünndarmobstruktion. Mit den Eltern wurde ein palliatives Vorgehen besprochen, der Junge verstarb am 7. Lebenstag in der progredienten Niereninsuffizienz.

Diskussion. Normalerweise kommt es bei fehlender intrauteriner Urinproduktion wie im Falle einer bilateralen Nierenagenesie zu einem Oligo-/Anhydramnion mit Entwicklung einer Lungenhypoplasie. Die Kombination aus einer Ebstein-Anomalie, einer intestinalen Obstruktion und einer bilateralen Nierenagenesie wurde bisher nicht beschrieben, ebenso nicht die Kombination einer bilateralen Nierenagenesie mit fehlendem Oligo-/Anhydramnion. Eine wesentliche protektive Rolle für die normale Lungenentwicklung dürfte die intestinale Obstruktion gespielt haben. Wir spekulieren daneben, dass es durch eine erhöhte Konzentration an ANP/BNP (Atrial/Brain Natriuretic Peptide) aufgrund der Dilatation des rechten Vorhofs bei Ebstein-Anomalie zu einer vermehrten Produktion von fetaler Alveolarflüssigkeit gekommen ist und so der Ausfall der renalen FW-Produktion kompensiert wurde. Schlussfolgerung. Sollte sich eine Regulation der intrauterinen FW-Produktion durch ANP/BNP bestätigen lassen, könnten sich hieraus und durch eine evtl. temporäre artifizielle intestinale Obstruktion ggf. weitreichende therapeutische Konsequenzen für die pränatale Behandlung von schweren Nierenfehlbildungen mit der Gefahr einer Lungenhypoplasie bzw. eines „dry lung syndrome“ ergeben.
P04-10

Erstbeschreibung einer PEX1 c.2226+T>C Mutation als Ursache eines Perioxisomen-Biogenesedefekts beim Zellweger-Syndrom

F. Mohr', A. Ohlenbusch², H. Rosewich², D. Horn³ , L. Garten', C. Bührer', J. Gärtner ${ }^{2}$, C. Dame ${ }^{1}$

'Charite-Universitätsmedizin, Klinik für Neonatologie, Berlin, Deutschland, 2Universitäts Klinik für Kinder-und Jugendmedizin, Neuropädiatrie, Göttingen, Deutschland, ${ }^{3}$ Charite-Universitätsmedizin, Institut für Medizinische Genetik und Humangenetik, Berlin, Deutschland

Hintergrund. Defekte der Peroxisomenbiosynthese infolge von $\mathrm{Mu}$ tationen im PEX-1 Genlocus (Peroxin 1; MIM 602136) bedingen sehr seltene, meist autosomal rezessiv vererbte metabolische Erkrankungen. Es werden zwei Gruppen der Erkrankung unterschieden: Gruppe 1, Peroxisomen-Biogenesedefekte (Zellweger-Syndrom, neonatale Adrenoleukodystrophie, infantiler Morbus Refsum), bei der die $\beta$-Oxidation überlangkettiger Fettsäuren, die Cholesterol- und Gallensäuresynthese oder der Abbau von Wasserstoffperoxiden gestört sind. Gruppe 2, bei der singuläre Enzymdefekte vorliegen (u. a. X-chromosomale Adrenoleukodystrophie).

Fallbericht. Wir stellen hier ein reifes weibliches Neugeborenes (GG 3360 g, GA 37 5/7; Apgar 3/6/6, NapH 7.23), konsanguiner türkischer Eltern $\left(\mathrm{G}_{2} / \mathrm{P}_{2}\right)$ vor. Pränatal Verdacht auf eine syndromale Erkrankung aufgrund polyzystischer Nieren. Unmittelbar postnatal generalisierte zerebrale Krampfanfälle mit zentralen Apnoen und Ateminsuffizienz. Beatmung für 2 Tage. Behandlung mit Phenobarbital, fortbestehende Anfallsaktivität im aEEG. Im cMRT-Nachweis einer bifrontoparietalen Pachygyrie mit Myelinisierungsverzögerung sowie einer Vermishypoplasie bei vergröbertem Kleinhirnrelief. Balkenhypoplasie mit fehlendem Splenium callosum bei normaler Anlage des Mesencephalons. Zusätzlich Molar-Tooth-Sign als Zeichen einer fehlgebildeten pontomesencephalen Verbindung. Klinisch korrelierten damit eine ausgeprägte generalisierte Muskelhypotonie, Schluckstörungen und zentrale Apnoen. Progrediente Niereninsuffizienz (max. Kreatinin 2,7 mg/dl) und Leberentzündung (max. GOT 2113 U/L, max. GPT 585 U/L). Deutlich erhöhte Werte für folgende Fettsäuren: 26:0 (11,9 nml/ml), 24:0/22:0 (1.85), 26:0/22:0 (o.714), fehlender Nachweis von 16:0 und 18:o Plasmalogenen in den Erythrozyten-Gesamtlipiden. Therapie-Begrenzung im Konsens mit den Eltern; das Mädchen verstarb am 29. Lebenstag.

Molekulare Diagnostik. Als molekulare Ursache des Zellweger-Syndroms wurde eine homozygote Spleiß-Mutation im Intron 13 des PEX1 Gens identifiziert (c.2226+2T>C), die bislang nicht in der Literatur beschrieben ist und zu einer Auslassung der Kodierung von Aminosäuren aus der Sequenz von Exon 13 und 14 führt (p.Ala691_Lys8o6delinsGlu). Daneben fanden sich drei bekannte homozygote Normvarianten: c.1804-144A $>\mathrm{T}$ (rs6465359), c.2331C $>\mathrm{A}$, pGly777Gly, Exon 14 (rs10278857), c.3439-59G>C (rs972923), die nicht als ursächlich anzusehen sind.

Schlussfolgerung. Im Rahmen der Syndromen-Abklärung waren die Leitsymptome für das Zellweger-Syndrom und die Stoffwechseldiagnostik für die Identifikation dieser neuen Mutation im PEX-1 Gen richtungsweisend. Die Mutation im Intron 13 führt zu einer gravierenden Störung der Kodierung im Bereich der Exone 13 und 14 und erklärt die extreme Ausprägung der Erkrankung. Basierend auf dieser Information kann den Eltern in einer folgenden Schwangerschaft eine gezielte pränatale Diagnostik angeboten werden. 


\section{Posterwalk 5 - Freie Themen 2}

\section{P05-01 \\ Beeinflusst ein Blasensprung von mindestens 7 Tagen das Outcome der betroffenen Frühgeborenen?}

T. Storbeck', N. Bruns', A. Stein', A. Köninger', U. Felderhoff-Müser', H. Müller' 'Universitäts-Kinderklinik Essen, Klinik für Kinderheilkunde I/Perinatalzentrum, Essen, Deutschland, ${ }^{2}$ Universitäts-Frauenklinik Essen, Essen, Deutschland

Hintergrund. Ein vorzeitiger Blasensprung (VBS) führt nicht selten dazu, dass die Indikation zur Sektio gestellt wird. Dies ist insbesondere der Fall, wenn die Infektionsparameter der Schwangeren ansteigen und man ein Amnioninfektionssyndrom beim Feten befürchten muss.

Fragestellung. Ziel ist es zu untersuchen, ob ein $V B S>7$ Tage ohne Hinweis auf ein Amnioninfektionssyndrom die neurologische Entwicklung der betroffenen Frühgeborenen beeinflusst.

Patienten und Methoden. Es wurden 26 Frühgeborene aus den Geburtsjahrgängen 2007-2013 untersucht, deren Mütter einen VBS von mindestens 7 Tagen aufwiesen. Diesen Kindern wurden im Rahmen einer Matched-pairs-Bildung jeweils Frühgeborene desselben Geburtsjahrgangs mit ähnlichem Geburtsgewicht und Gestationsalter, aber ohne VBS zugeordnet. Beide Gruppen (jeweils 26 Frühgeborene) wurden bezüglich der Komplikationen während des postnatalen Verlaufs sowie hinsichtlich der neurologischen Entwicklung verglichen.

Ergebnisse. Die beiden Gruppen zeigten ein identisches mittleres Geburtsgewicht [VBS-Kinder: $1358 \mathrm{~g}$ (630-2940 g); Vergleichskinder: $1353 \mathrm{~g}$ (660-2940 g)] und ein identisches mittleres Gestationsalter [VBS-Kinder: 28,8 vollendete SSW (24-36 SSW); Vergleichskinder: 28,9 vollendete SSW (24-36 SSW)]. Bei den VBS-Kindern betrug die mittlere Blasensprung-Dauer 412 Stunden (170-1248 Stunden), das entspricht 17,2 Tagen (7,1-52 Tage), und bei den Kindern der Vergleichsgruppe o,8 Stunden (o-10 Stunden). Das mütterliche CRP bei Geburt betrug im Mittel 1,47 mg/dl (o-5,4 mg/dl) bei der VBS-Gruppe und 3,6 mg/dl (o,1-24,3 mg/dl) bei der Vergleichsgruppe. Die Kinder der VBS-Gruppe hatten in den ersten 3 Lebenstagen (LT) ein mittleres maximales CRP von $0,34 \mathrm{mg} / \mathrm{dl}(0-1,5 \mathrm{mg} / \mathrm{dl})$, während es bei Vergleichsgruppe $0,8 \mathrm{mg} /$ $\mathrm{dl}(\mathrm{o}-1,4 \mathrm{mg} / \mathrm{dl})$ betrug. Allerdings wiesen die VBS-Kinder ein höheres mittleres IL-6 in den ersten 3 Tagen auf (210 pg/ml versus 23,2 pg/ml). 6 Kinder hatten jeweils eine IVH (VBS-Gruppe: 3-mal IVH 1; 2-mal IVH 2; 1-mal IVH 3; Kontrollgruppe: 4-mal IVH 1; 1-mal IVH 2; 1-mal IVH 3). Jeweils 2 Kinder zeigten in beiden Gruppen eine BPD im Alter von 36 SSW. Der Bayley-Test im korrigierten Alter von 24 Monaten betrug für die 9 getesteten VBS-Kinder im Mittel 97,1 (MDI-Score) und 97 (PDIScore) und für die 10 getesteten Vergleichskinder 100 (MDI-Score) und 84,2 (PDI-Score). Fünf Kinder der VBS-Gruppe verstarben an folgenden Ursachen: respiratorisches Versagen bei Pneumothoraces (25 SSW, 1. LT), schwere IVH (25 SSW, 4. LT), Late-onset-Sepsis (24 SSW, 14. LT), PPHN (24 SSW, 1. LT) und Lungenhypoplasie (29 SSW, 1. LT). In der Vergleichsgruppe verstarb ein Kind im unklaren kardiogenen Schock bei Verdacht auf Infektion (25 SSW, 2. LT).

Schlussfolgerung. Ein zuwartendes Verhalten bezüglich der Sektio-Indikation bei einem VBS von mehr als 7 Tagen, niedrigen maternalen Infektwerten und noch ausreichender Fruchtwassermenge ist gerechtfertigt.

\section{P05-02}

\section{Medizinische Probleme von Very-low-birth-weight-Frühgebore-} nen im Alter von 5 Jahren

\section{J. Spiegler', F. Pagel', E. Herting ${ }^{3}$, W. Göpel ${ }^{4}$}

'Universitätsklinikum Schleswig Holstein, Kinderklinik, Lübeck, Deutschland, ${ }^{2}$ Universitätsklinik Lübeck, Pädiatrie, Lübeck, Deutschland, ${ }^{3}$ Universitätsklinikum Schleswig-Holstein Campus Lübeck, Direktor der Klinik für Kinder- und Jugendmedizin, Lübeck, Deutschland, ${ }^{4}$ Universitätsklinikum Schleswig-Holstein, Klinik für Kinder- und Jugendmedizin, Lübeck, Deutschland

Hintergrund. Daten über chronische gesundheitliche Probleme von ehemaligen VLBW Frühgeborenen sind nur unzureichend vorhanden.

Fragestellung. Wir wollten wissen, ob ehemalige VLBW Frühgeborene sich im Hinblick auf chronische Erkrankungen oder Operationen von einer nationalen Kohorte (KIGGS) unterscheiden.

Material und Methoden. Im Rahmen einer multizentrischen Studie von VLBW Frühgeborenen erfolgte im Alter von 5 Jahren ein persönliches Elterninterview analog zur Durchführung in der KIGGS-Studie. 225 Kinder wurden bis Januar 2015 nachuntersucht [Geburtsgewicht in Gramm (median, Q1-Q3): 1113 (820-1380); Gestationsalter in Wochen (median, Q1-Q3): 29 (27-31)] und mit 945 Kindern im gleichen Alter aus der KIGGS-Studie verglichen.

Ergebnisse. Ehemalige Frühgeborene litten seltener unter Neurodermitis ( $8 \%$ vs. $13 \%$ ) oder allergischer Rhinitis (1\% vs. $6 \%)$, sie unterschieden sich nicht signifikant im Auftreten von Asthma (4\% vs. $3 \%$ ) oder Diabetes (0.4\% vs. 0.1\%). Es fand sich eine gering erhöhte Rate Migräne ( $1 \%$ vs. O\%), sowie deutlich erhöhte Rate an generalisierten Krampfanfällen (inklusive Fieberkrämpfen; $8 \%$ vs. $3 \%$ ), Herzerkrankungen ( $8 \%$ vs. $2 \%$ ) und obstruktiven Bronchitiden (48\% vs. 17\%). Ehemalige VLBW Früh-

Tab. P05-02 Chronische Erkrankungen und Operationen

\begin{tabular}{|llll} 
& VLBW & KIGGS & p-Wert \\
\hline N=225 & N=945 & 0,0033 \\
\hline Heuschnupfen & $3(1 \%)$ & $56(6 \%)$ & 0,0371 \\
\hline Neurodermitis & $17(8 \%)$ & $120(13 \%)$ & 0,6861 \\
\hline Asthma & $9(4 \%)$ & $32(3 \%)$ & $<0,0001$ \\
\hline Obstruktive Bronchitis & $109(48 \%)$ & $162(17 \%)$ & 0,0004 \\
\hline Herzerkrankungen & $18(8 \%)$ & $24(2 \%)$ & 0,031 \\
\hline Siabetes & $17(8 \%)$ & $28(3 \%)$ & 0,3478 \\
\hline Migräne & $1(0,4 \%)$ & $1(0,1 \%)$ & 0,0368 \\
\hline Jemals OP & $2(1 \%)$ & 0 & $<0,0001$ \\
\hline Adenotomie & $114(50 \%)$ & $306(32 \%)$ & 0,68 \\
\hline Tonsillektomie & $32(14 \%)$ & $148(16 \%)$ & 0,45 \\
\hline Paukenröhrchen & $11(5 \%)$ & $36(4 \%)$ & 0,0007 \\
\hline Appendektomie & $30(13 \%)$ & $59(6 \%)$ & 1,0 \\
\hline Zirkumzision & 0 & $4(0,4 \%)$ & $<0,0001$ \\
\hline
\end{tabular}


geborene wurden häufiger operiert ( $50 \%$ vs. $32 \%$ ), insbesondere für eine Paukenröhrchenanlage ( $13 \%$ vs. $6 \%$ ), während Zirkumzisionen (o\% vs. $7 \%$ ) seltener erfolgten (Tab. Po5-O2).

Diskussion. Im Altern von 5 Jahren litten ehemalige VLBW Frühgeborene signifikant häufiger unter obstruktiven Bronchitiden und erhielten häufiger Paukenröhrchen als Kinder gleichen Alters in der KIGGS Kohorte. Allergische Erkrankungen wie Neurodermitits und Heuschnupfen traten seltener auf.

\section{P05-03}

Potential der Beschleunigungsmessung zur Frühdiagnose von Bewegungsstörungen

C. Bultmann', M. Schoberer', S. Trepels-Kottek', C. Disselhorst-Klug², C. Wilkmann $^{2}$, D. Jesch' ${ }^{2}$, T. Orlikowsky'

'Uniklinik RWTH Aachen, Klinik für Kinder- und Jugendmedizin, Sektion Neonatologie, Aachen, Deutschland, ${ }^{2}$ Institut für Angewandte Medizintechnik der RWTH Aachen, Lehr- und Forschungsgebiet Rehabilitations- \& Präventionstechnik, Aachen, Deutschland

Hintergrund. Auf dem von Lorenz begründeten Prinzip der Gestaltwahrnehmung beruht das General Movement Assessment (GMA) nach Prechtl. General Movements (GM) sind ungerichtete Bewegungen von Kopf, Rumpf, Armen und Beinen, die zwischen 10 und 6o Wochen postmenstruellem Alter (wPMA) auftreten. Bei regelrechter psychomotorischer Entwicklung lassen sich die GM in drei Phasen unterteilen: „preterm movements" (PM, ab 10 wPMA), „writhing movements" (WM, 34-48 wPMA) und "fidgety movements" (FM, 49-60 wPMA). Mittels GMA kann in der Phase der FM die spätere Entwicklung einer Cerebralparese (CP) prognostiziert werden. Ähnlich sind selbstlernende Algorithmen geeignet, Bewegungsinformationen z. B. aus Beschleunigungsmessungen (Akzelerometrie) auszuwerten. In einer früheren Publikation konnten wir zeigen, dass auch die Akzelerometrie geeignet ist, um mit 3-5 Monaten eine $\mathrm{CP}$ vorherzusagen.

Fragestellung. Das GMA hat in der Phase der FM zwischen dem 3. und 5. Lebensmonat die höchste prädiktive Validität für eine spätere $\mathrm{CP}$, weshalb sich auch Evaluationen automatisierter Verfahren bislang auf dieses Zeitfenster konzentrieren. Ob diese Verfahren zu früheren Zeitpunkten bereits spezifische Normabweichungen detektieren können, ist nicht untersucht. Ziel dieser Studie ist es deshalb, an Late Preterm Infants (LPI) erstmalig die Bewegungsabläufe in der (früheren) Phase der WM messtechnisch zu erfassen und deren Spezifika von denen in der Phase der FM abzugrenzen.

Material und Methoden. Nach positivem Ethik-Votum führten wir eine prospektive Beobachtungsstudie an $n=23$ LPI mit komplikationslosem postnatalem Verlauf durch. (Reifealter bei Geburt: $>32$ o/7 wPMA insb. keine Asphyxie, IVH $>^{\circ}$ I, PVL, NEC, BPD). Es erfolgten Messungen mit 35, 36 (Phase der WM) sowie 52 wPMA (Phase der FM). Die Beschleunigungsmessung erfolgte mit 5 triaxialen Beschleunigungssensoren, welche über einen $\mathrm{AD}$-Wandler eingelesen, mit einem Microcontroller verarbeitet und über USB mit einem PC verbunden wurden. Pro Messtermin wurden fünf zweiminütige Messungen aufgezeichnet (insg. $\mathrm{n}=303$ auswertbare Messungen). Aus den Sensorsignalen wurden 34 Parameter extrahiert (direkte Messgrößen u. Größen der deskriptiven Statistik), die die Bewegungsabläufe quantitativ beschreiben. Die Signifikanztestung erfolgte mittels Mann-Whitney-U-Test auf einem Niveau von $\mathrm{p}<0,05$.

Ergebnisse. Es bestanden signifikante Unterschiede bei 19 von 34 Parametern. Die mediane Beschleunigung der Hände und Füße war während der FM höher als während der WM (4,5 bzw. 3,6-fach, p<o,ooo1). Zudem wies das Beschleunigungsprofil der FM's einen niedrigeren Kreuzkorrelationskoeffizienten auf. Das entspricht der Beobachtung, dass die Variabilität in den Bewegungsabläufen zwischen WM und FM zunimmt. Bereits zwischen 35 und 36 wPMA (beide WM) beobachteten wir für drei Parameter statistisch signifikante Unterschiede (Abb. Po503).
Vergleich der medianen Geschleunigung der Hände und Füße in der Phase der writhing und fidgety movements

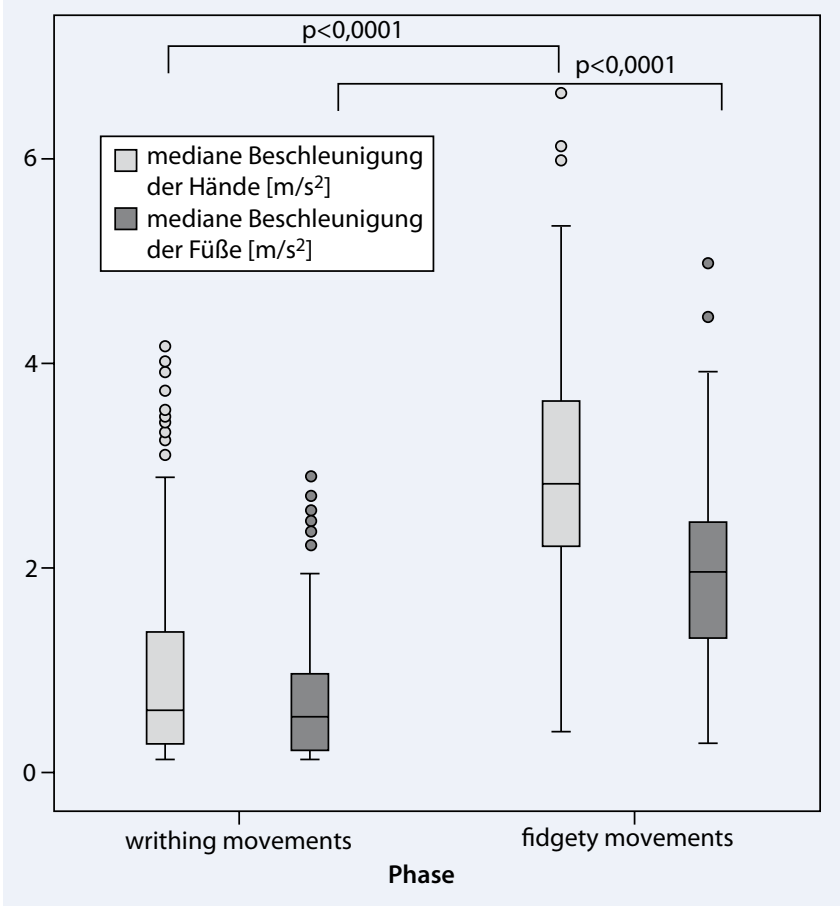

Abb. P05-03 $\triangle$

Diskussion. Messtechnische Verfahren bieten gegenüber dem GMA den theoretischen Vorteil einer höheren räumlichen und zeitlichen Auflösung, der Reproduzierbarkeit und der Beobachter-Unabhängigkeit. Die klare Abgrenzbarkeit von WM und FM in unseren Messungen bestärkt uns in der Erwartung, dass eine Diskriminierung geringerer Unterschiede schon zu diesem frühen Zeitpunkt möglich ist. Dafür könnten auch die (weniger eindeutigen) Unterschiede zwischen der 35- und 36-Wochen-Messung sprechen. Die prädiktive Validität solcher Unterschiede bedarf weiterer Untersuchungen.

\section{P05-04}

Analyse klinischer Einflussfaktoren auf die Länge der stationären Verweildauer von Frühgeborenen im Swiss Neonatal Network

S. Wellmann', C. Rüegger' ${ }^{2}$ M. Adams'2, S. Schulzke ${ }^{1}$

'Universitäts-Kinderspital beider Basel (UKBB), Basel, Schweiz, ${ }^{2}$ Klinik für Neonatologie, UniversitätsSpital Zürich, Zürich, Schweiz

Hintergrund. Je unreifer ein Kind bei der Geburt und je mehr schwere Komplikationen auftreten, desto länger ist seine stationäre Verweildauer. Erst wenn spezifische physiologische Reifekriterien erreicht sind, ist die Entlassung nach Hause möglich. Da sich die Vergütung nach Fallpauschalen nach Geburtsgewicht, operativen Prozeduren und stationärer Verweildauer richtet, ist es von zentraler Bedeutung, den Zusammenhang dieser drei Parameter zu kennen.

Fragestellung. Welchen Einfluss haben schwere Komplikationen, operative Prozeduren und Mortalität auf die stationäre Verweildauer von sehr frühgeborenen Kindern? Gibt es Unterschiede dieser Parameter zwischen den neuen Behandlungszentren in der Schweiz?

Material und Methoden. Retrospektive Analyse eines prospektiv erhobenen Datensatzes des Swiss Neonatal Network aller in der Schweiz von 2006 bis 2012 lebendgeborenen Kinder mit mindestens 24 Wochen Gestationsalter (GA) bei Geburt und einem Geburtsgewicht von kleiner $1500 \mathrm{~g}$ und/oder einem GA bei Geburt von weniger als 32 Wochen 
$(\mathrm{n}=5622)$. Multivariable lineare Regressionsanalyse der Kinder mit Überleben bis mindestens zur Entlassung nach Hause $(n=5022)$ und aller Kinder einschließlich der verstorbenen $(\mathrm{n}=5622)$ mittels kombinierter Analyse der Überlebenszeit und stationären Verweildauer nach Kaplan-Meier, multivariable Cox-Regressionsanalyse.

Ergebnisse. Im Median erfolgte die Entlassung nach Hause mit 37.9 Wochen postmenstruales Alter (PMA). Unabhängig von anderen klinischen Faktoren wie Geburtsgewicht, Gestationsalter bei Geburt und Mortalität, hatte die moderate bis schwere Bronchopulmonale Dysplasie (BPD, Diagnose mit 36 Wochen PMA, $n=487,8,7 \%$ ) den größten Einfluss auf die stationäre Verweildauer. Im Einzelnen verlängerte sich der stationäre Aufenthalt signifikant und unabhängig bei moderater bis schwerer BPD um 24 Tage, bei Herzchirurgie um 12 Tage $(n=184$, $3,3 \%)$, bei grosser abdomineller Chirurgie um 12 Tage $(n=245,4,4 \%)$, bei relevanter Nekrotisierender Enterokolitis (NEC) und/oder Fokaler intestinaler Perforation (FIP) um 7 Tage $(\mathrm{n}=190,3,4 \%)$, bei höhergradiger Hirnblutung und/oder höhergradiger Leukomalazie um 5 Tage $(n=590$, 10,5\%), bei Spätsepsis um 4 Tage $(n=464,8,3 \%)$ und bei Transfer in eine sekundäre Neonatologie um 3 Tage $(n=1886,33,5 \%)$. Zwischen den neun primären Behandlungszentren zeigten sich signifikante und unabhängige Unterschiede von bis zu 11 Tagen in der stationären Verweildauer, welche nicht durch Unterschiede in der Mortalitätsrate, sondern eher durch unterschiedliche Entlassungsstrategien erklärt werden können. Diskussion. In der Vergütung nach Fallpauschalen bekommt die Länge der stationäre Verweildauer zunehmend mehr Gewicht im Vergleich zu operativen Prozeduren. Damit werden die Vermeidung von Komplikationen wie die BPD und ein effizientes Austrittsmanagement immer wichtiger. Für eine umfassende Analyse und bessere Vergleichbarkeit zwischen den Behandlungszentren sollte in Zukunft auch die Rehospitalisation im ersten Lebensjahr erfasst werden.

\section{P05-05}

\section{Status der intestinalen Perfusion des Fetus unter der Geburt}

D. Mensinga', D. Katzer', A. Heydweiller', H. Reutter', P. Bartmann', A. Müller', S. Bagci'

'Zentrum für Kinderheilkunde, Universität Bonn, Neonatologie und Pädiatrische Intensivmedizin, Bonn, Deutschland, ${ }^{2}$ Chirurgisches Zentrum, Universitätsklinikum Bonn, Kinderchirurgie, Bonn, Deutschland

Hintergrund. Uterine Kontraktionen unter der Geburt können zu einer intermittierenden uteroplazentaren Hypoperfusion und so zu Ischämie-/Reperfusionsschäden führen. Aufgrund anatomischer Besonderheiten ist die intestinale Mukosa für hämodynamische Änderungen anfällig. Bereits kurze Phasen der Hypoperfusion führen zu einer Störung ihrer Barrierefunktion mit gesteigerter Permeabilität. Das humane intestinale Fettsäure-bindende Protein (I-FABP) ist ein zytosolisches Protein im Mukosaepithel mit niedrigem Molekulargewicht (14-15 $\mathrm{kDa}$ ). Viele Studien weisen drauf hin, dass eine erhöhte I-FABP-Konzentration im Blut/Urin ein sensitiver Biomarker für eine Schädigung der intestinalen Mukosazellen ist. Die antioxidativen Enzyme Superoxiddismutase (SOD) und Glutathion-Peroxidase (GPx) schützen die Zellen und Gewebe gegen Reperfusionsschäden durch freie Radikale.

Fragestellung. Das Primärziel dieser Studie war es, die Effekte des Entbindungsmodus auf die intestinale Perfusion des Fetus unter der Geburt zu evaluieren. Zusätzlich wurde die Beziehung zwischen den antioxidativen Enzymen SOD und GPX und intestinaler Schädigung untersucht.

Material und Methoden. Insgesamt wurden 80 gesunde Neugeborene, Gestationsalter 38 (34-42) Schwangerschaftswochen (Median, Spannweite), in die Studie eingeschlossen. Das Patientenkollektiv wurde nach Geburtsmodus [Spontanpartus (SP), $\mathrm{n}=30$, elektive Sectio ohne Wehen (eCS), n=50] eingeteilt. IFABP, 8-Hydroxydesoxyguanosin, SOD und GPx (jeweils mit ELISA), sowie arterieller $\mathrm{pH}(\mathrm{apH})$ und Base Excess (aBE), wurden im arteriellen Nabelschnurblut (NA) bestimmt.

Ergebnisse. Im Vergleich zur eCS-Gruppe fanden wir in der SP-Gruppe statistisch signifikant höhere IFABP-Konzentration [Median (Inter- quartilsabstand); $367 \mathrm{pg} / \mathrm{ml}(250-484)$ vs. $541 \mathrm{pg} / \mathrm{ml}(398-863) \mathrm{p}<0,001]$. Es bestand eine statistisch signifikante negative Korrelation zwischen IFABP-Konzentration und APGAR-Wert nach einer Minute $(\mathrm{r}=-0,284$; $\mathrm{p}=0,011)$, apH $(\mathrm{r}=-0,337 ; \mathrm{p}=0,002)$ sowie $\mathrm{aBE}(\mathrm{r}=-0,352 ; \mathrm{p}=0,001)$. Eine signifikante positive Korrelation fand sich zwischen IFABP-, SOD- $(r=0,509 ; p<0,001)$ und GPx-Konzentration $(r=0,274 ; p=0,012)$. Außerdem wurde eine signifikant negative Korrelation zwischen aBE, SOD- $(\mathrm{r}=-0,383 ; \mathrm{p}=0,001)$ und GPx-Konzentration $(\mathrm{r}=-0,323 ; \mathrm{p}=0,004)$ nachgewiesen, sowie eine signifikant positive Korrelation zwischen $\mathrm{aBE}$ und 8-OHdG-Konzentration $(\mathrm{r}=-0,265 ; \mathrm{p}=0,021)$. Es gab keine signifikante Korrelation zwischen 8-OHdG- Konzentration und IFABP$(\mathrm{r}=-0,190 ; \mathrm{p}=0,107)$, SOD- $(\mathrm{r}=-0,214 ; \mathrm{p}=0,063)$ oder GPx-Konzentration $(\mathrm{r}=-0,100 ; \mathrm{p}=0,389)$.

Schlussfolgerung. Die Ergebnisse zeigen, dass die intestinale Perfusion des Fetus unter SP mehr beeinträchtigt ist als unter eCS. Ein Anstieg der SOD- und GPx-Konzentrationen könnte einen protektiven Mechanismus für die unter Spontanpartus bevorstehenden intestinalen Perfusionsstörungen darstellen.

\section{P05-06}

Multidimensional response to vaccination pain in very preterm, moderate- to-late preterm and full-term infants at age three months

K. Mehler', L. Ulbrich', S. Börner', A. Joachim', I. Becker', B. Roth', C. Hünseler' 'University Hospital of Cologne, Department of Neonatology, Cologne, Deutschland, ${ }^{2}$ University of Cologne, Institute of Medical Statistics, Informatics and Epidemiology, Cologne, Deutschland

Background. Very early life pain exposure and stress induces alterations in the developing brain and leads to altered pain sensitivity. In premature infants with a history of numerous early postnatal adverse events, behavioral responsiveness and hypothalamic-pituitary-adrenal (HPA) axis reactivity may show alterations as well.

Aims. We compared a multidimensional response to a painful situation (vaccination) in three month old infants. The study involved very preterm, moderate to late preterm infants and full-term infants with varying exposure to pain and stress within the first weeks of life.

Study design. At the age of three months, we evaluated the infants' reactivity to intramuscular injections for immunization.

Subjects. The study included 61 very preterm infants, 30 moderate to late preterm infants and 30 full-term infants.

Outcome measures. We assessed heart rate recovery, Bernese pain Score and increase of salivary cortisol following vaccination. We also evaluated the flexor withdrawal reflex threshold as well as Prechtl's General Movements. Secondly, we assessed factors potentially influencing pain reactivity such as exposure to pain/stress, gender, use of steroids or opioids and mechanical ventilation.

Results. Very preterm, moderate to late preterm and full-term infants showed different reactivity to pain in all analyzed aspects. Very preterm infants showed a lower level of behavioral and physiologic reactivity and exposure to pain/stress predicted lower cortisol increase.

Conclusion. At three months of age, very preterm infants show an altered level of HPA axis reactivity. Efforts aiming at minimizing pain and stress in premature infants should be taken. 


\section{P05-07}

Increased rate of parental postpartum depression and traumatisation in moderate and late preterm infants is independent of the infant's motor repertoire

\section{K. Mehler', A. Mainusch', E. Hucklenbruch-Rother', M. Hahn', C. Hünseler',} A. Kribs

'University of Cologne Children's Hospital, Department of Neonatology, Cologne, Deutschland, ${ }^{2}$ University of Cologne, Institute of Medical Statistics, Informatics and Epidemiology, Cologne, Deutschland

Background. Moderately and late preterm infants represent a considerable and increasing proportion of infants cared for in neonatal departments worldwide. Preterm infants parents are at risk for postpartal depression (PPD) and traumatization (PTSD) and preterm infants are at risk for developmental impairment.

Aim. To assess the correlation of parental PPD and PTSD and infants' neurologic abnormalities and illness severity.

Subjects. We studied 60 mothers and 56 fathers of 69 preterm infants (born at 32 to 37 weeks of gestation) and 32 mothers and 29 fathers of 34 fullterm infants. We assessed parental PPD, PTSD and perceived social support and infants' neurologic development at birth, term and three months corrected age.

Results. Preterm mothers and fathers had significant higher depression scores after birth compared to fullterm parents ( $\mathrm{p}=0.03$ and o.02). Preterm fathers also had higher traumatization scores compared to fullterm fathers $(\mathrm{p}<0.01)$. Probable or possible PPD/PTSD was not correlated to infant's illness severity. No differences in neurologic development were found between preterm and fullterm infants.

Conclusion. Moderate to late preterm infants' parents are at increased risk for PPD irrespective of infants' neurologic development or illness severity.

\section{P05-08}

\section{Massive Hyperleukozytose bei einem Neugeborenen mit com- mon-B-ALL, CD 33+:}

\section{U. Baron', C. Denne', M. Walka'}

${ }^{1}$ Klinikum Ludwigsburg, Kinderklinik, Ludwigsburg, Deutschland

Anamnese und Befund. Bei reifem Neugeborenem mit silentem CTG und grünem Fruchtwasser erfolgte eine eilige Sectio. Entbunden wurde ein männlicher Säugling mit 3800 g Geburtsgewicht, schlaff, ohne Spontanatmung, mit disseminierten fleckförmigen Einblutungen.

Diagnostik. Nabelarterien-pH 6,9, BE -14. Leukozyten max 552.00o/ $\mu 1$,

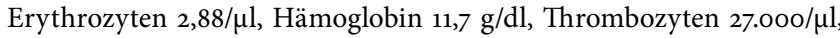
Retikulozyten 1,5\%, CRP neg., IL-6 278 pg/ml, LDH 1396 U/l, GOT 316 U/l, GPT 175 U/l, Harnsäure 11,3 mg/dl, Kreatinin 1,09 mg/dl, Quick 42\%, Blutausstrich: Zahlreiche lymphozytäre Blasten nachweisbar. Röntgen-Thorax: Herzschatten vergrößert. Teils streifige, teils flächige Verdichtung in beiden Oberfeldern. Sonographie Schädel: Generalisierte Echogenitätsvermehrung, vor allem am Interhemisphärenspalt.

Klinischer Verlauf und Therapie. Es erfolgte eine primäre Intubation und eine Therapie mit Ampicillin, Gentamicin und Cefotaxim. Der Patient erhielt 1 FFP, 1 Erythrozyten- und 2 Thrombozytenkonzentrate sowie eine Therapie mit Phenobarbital und Levetiracetam bei Krampfanfällen am ersten Lebenstag. Bei Verdacht auf konnatale Leukämie wurde der Patient in ein kinderonkologisches Zentrum verlegt. Dort erfolgte vor der Induktionstherapie eine Austauschtransfusion zur Verminderung polzytämischer Komplikationen. Trotzdem erlitt der Patient einen ischämischen Insult. Aus dem Knochenmark wurde eine common-BALL mit $\mathrm{CD}_{33}$-Koexpression diagnostiziert und eine Chemotherapie nach Interfant o6-Protokoll begonnen. Der Patient wurde wegen eines MLL-Rearrangement, hoher Leukozytenzahl und schlechten Ansprechens auf Prednisolon in die Hochrisikogruppe eingeteilt. Nachdem durch die Induktionstherapie keine Remission erreicht wurde, erfolgte im Konsens mit den Eltern im Weiteren eine palliative Therapie. Zuletzt war der Patient ohne Atemhilfe stabil. Neonatale Leukämie: Inzidenz: 1-5:1.00o.ooo Lebendgeburten. Symptome: Hyperleukozytose, Hepatosplenomegalie, Leukemia cutis, Anämie, Thrombozytopenie, Blutungsneigung, meningeale Infiltration, Krampfanfälle. Differentialdiagnosen: Konnatale Infektionen (Sepsis, TORCH), Leukämoide Reaktion (Hypoxie, Hämolyse), andere Neoplasien (transiente myeloproliferative Erkrankung, Neuroblastom, Langerhanszell-Histiozytose). Diagnostik: Differentialblutbild, Blutausstrich, Knochenmarkpunktion. Therapie: ALL nach Interfant 06-Protokoll. Prognose: Bei ALL 17\% Langzeitüberlebensrate, $87 \%$ Remission, Rückfallquote jedoch bei $73 \%$. Bei AML liegen größere Fallzahlen vor, die Überlebensrate wird auf 25\%, die Rückfallquote auf $50 \%$ geschätzt.

Schlussfolgerung. Die neonatale Leukämie ist eine sehr seltene Erkrankung mit ungünstiger Prognose. Unser Patient zeigte eine ungewöhnlich starke Hyperleukozytose mit Komplikationen. Entscheidend ist eine rasche Diagnosestellung und vitale Stabilisierung, um die Verlegung in ein kinderonkologisches Zentrum zu ermöglichen.

\section{P05-09}

Blood sampling via a peripheral artery catheter decreases cerebral oxygenation index in very low birth weight infants

\section{K. Mehler', M. Nowak ${ }^{2}$, A. Oberthuer ${ }^{3}$, M. Hahn ${ }^{4}$, B. Roth', A. Kribs'}

'University of Cologne Children's Hospital, Department of Neonatology,

Cologne, Deutschland, ${ }^{2}$ Düren Community Hospital, Department of Cardiology, Düren, Deutschland, ${ }^{3}$ University of Cologne Children's Hospital, Department of Neonatology, Cologne, Deutschland, ${ }^{4}$ University of Cologne, Institute of Medical Statistics, Informatics and Epidemiology, Cologne, Deutschland

Aim. This study evaluated the impact of blood sampling via peripheral arterial catheters on cerebral oxygenation and blood volume as a function of blood sampling velocity.

Methods. Near infrared spectroscopy was applied to 20 very low birth weight infants during peripheral arterial blood sampling. Changes in cerebral oxygenated, deoxygenated and total haemoglobin, cerebral blood volume and cerebral oxygenation index were recorded. Heart rate and oxygen saturation were measured continuously. To assess the impact of blood sampling velocity, both fast 40 second and slow 70 second sampling procedures were performed in a cross-over study design, in which the order of sampling velocities was randomised for each patient. Results. Both fast and slow blood sampling procedures resulted in a significant decrease in cerebral oxygenation index (fast, $p=0.002$, slow, $\mathrm{p}=0.008)$, and an increase in mean heart rate (both $\mathrm{p}=0.02)$ and mean blood pressure ( $\mathrm{p}=0.02$ and 0.04$)$. Oxygenated and total haemoglobin and cerebral blood volume only decreased significantly after slow blood sampling $(\mathrm{p}<0.001)$.

Conclusion. Blood sampling from peripheral arterial catheters leads to significant fluctuations in cerebral oxygenation independent of the sampling velocity. Changes are comparable to those reported from umbilical blood sampling. We advise that blood sampling should be restricted as much as possible. 
P05-10

Nahinfrarotspektroskopie (NIRS) zur Messung der cerebralen Sauerstoffsättigung bei Frühgeborenen (FG) mit persistierendem Ductus arteriosus Botalli (PDA): eine systematische Literaturübersicht

\section{Schwarz', F. Neunhoeffer'2, D. Bassler', C. Poets', A. Franz'}

'Eberhard-Karls-Universität Universitätsklinik für Kinderheilkunde und Jugendmedizin, Neonatologie, Tübingen, Deutschland, ${ }^{2}$ Eberhard-KarlsUniversität Universitätsklinik für Kinderheilkunde und Jugendmedizin, Kinderkardiologie, Pulmologie und pädiatrische Intensivmedizin, Tübingen, Deutschland, ${ }^{3}$ UniversitätsSpital Zürich, Klinik für Neonatologie, Zürich, Schweiz

Hintergrund. Der PDA bei FG ist mit erhöhter Morbidität (z. B. intraventrikulären Blutungen) und Mortalität assoziiert. Pathophysiologisch ist zu erwarten, dass der Links-Rechts-Shunt bei PDA die cerebrale Perfusion kompromittiert und zu einer reduzierten cerebralen Sauerstoffsättigung ( $\left.\mathrm{rcStO}_{2}\right)$ oder einer erhöhten Sauerstoffausschöpfung (rcFtOE) führt, welche mittels NIRS bestimmbar wäre. Die vorliegenden Definitionen eines hrPDA sind allerdings umstritten.

Fragestellung. Zeigen klinische Studien bei FG mit PDA eine verminderte $\mathrm{rcStO}_{2}$ oder erhöhte rcFtOE?

Material und Methoden. Literatursuche in Pubmed (1994 bis 03/2014) mit den Suchbegriffen „ductus“ und „spectroscopy“ (Limits: humans, infant). Die gefundenen Abstracts wurden hinsichtlich klinischer Relevanz von 2 Autoren unabhängig geprüft und bei Konsens im Volltext bewertet. Einschlusskriterien waren: Originalpublikation einer klinischen Studie (keine Reviews, keine Einzelfallbeschreibungen), Sprache Englisch oder Deutsch. Die Ergebnisse sind als Mittelwert (Standardabweichung; ggf. Spannweite) angegeben.

Ergebnisse. Von den initial 46 Treffern erfüllten 3 Beobachtungsstudien die Einschlusskriterien. Es wurden INVOS 4100 bzw. 5100 verwendet, welche relative Veränderungen, jedoch keine Absolutwerte bestimmen können. Nur in einer Studie wurde der Sensortyp berichtet. In Studie A \& C wurde der Sensor frontal, in B frontoparietal platziert. Die hrPDA Definitionen waren verschieden und wurden in $C$ nicht detailliert berichtet. A: untersuchte primär unsere Fragestellung an 24 FG mit großem PDA ( $>3 \mathrm{~mm})$ vs. 14 FG mit moderatem PDA (1,5-3 mm): $\mathrm{rcStO}_{2}$ $68, \mathrm{o}(5,8) \%$ vs. $68,3(8,1) \%$; $\mathrm{p}=\mathrm{o}, 8 \mathrm{o}$; rcFtOE o,255 (o,o86) vs. o, $263(0,107)$; $\mathrm{p}=0,83$ [Geburtsgewicht (GG) 913 (207)g; Gestationsalter (GA) 26,8 (2,o) Wochen (w); postnatales Alter von $8(7) \mathrm{d}]$. Eine Kontrollgruppe ohne PDA wurde nicht untersucht. In 2 weiteren Therapie-Studien wurden Daten zur Oxygenierung vor Intervention publiziert: B: 20 FG mit PDA vs. 20 FG ohne PDA (matched controls): $\mathrm{rcStO}_{2} 62$ (10)\% vs. 72 (11)\%; $\mathrm{p}<0,05$ und rcFtOE $0,34(0,10)$ vs. o,25 (0,10); p<0,05 [GG 1154,5 (242)g; GA 28(1,5)w; postnatales Alter 1,7 (o,71-3,17) d]. Hierbei wurde jedoch nur in $<50 \%$ der Kontrollgruppe eine echokardiografische Kontrolle durchgeführt. C: 12 FG ohne vs. 33 FG mit hrPDA: $\mathrm{rcStO}_{2} 69$ (4)\% vs. 66 (10)\% und rcFtOE 0,28 (0,04) vs. 0,30 (0,09) [GG 830 (162)g; GA 26(1) w; postnatales Alter hrPDA-Gruppe 9,7 $(7,1) \mathrm{d})$ ]. In B und $\mathrm{C}$ wurden die PDA-Dimensionen nicht angegeben bzw. in die Auswertung einbezogen. Auf eine zusammenfassende statistische Auswertung wurde bei hoher Variabilität der Studien verzichtet.

Schlussfolgerung. Auch wenn der Einfluss eines PDA auf die $\mathrm{rcStO}_{2}$ und rcFTOE zu erwarten ist, konnte diese systematische Literaturübersicht den Beleg dazu nicht erbringen. Die Standardisierung von Technik und Definition sind notwendige Voraussetzungen für zukünftige aussagekräftige Studien.

\section{Posterwalk 6 - Pulmonologie}

\section{P06-01}

Gegensätzliche Wirkung von Progesteron und Steroide auf die Surfactant Protein B (SP-B) Synthese

S. Kunzmann', M. Fehrholz', B. Kramer' ${ }^{2}$, C. Speer ${ }^{7}$

'Universitäts-Kinderklinik, Würzburg, Deutschland, ${ }^{2}$ Maastricht University Medical Center, Department of Pediatrics, Maastricht, Niederlande

Hintergrund. Progesteron besitzt eine schwangerschaftsverlängernde Wirkung und soll die perinatale Morbidität senken. Neben seiner lokalen Wirkung mit Hemmung der Cervixreifung und Reduzierung der Kontraktilität des Myometriums wird für Progesteron eine Modulation von Inflammationsmechanismen diskutiert. Der Einfluss von Progesteron auf die Surfactant Homeostase in der Lunge ist bisher nur wenig untersucht.

Methodik. Die Expression von SP-B wurde mittels real time quantitative PCR und Western-blot-Analyse in Lungenepithel Zellen (H441) untersucht, die mit Progesteron (1o $\mu \mathrm{g} / \mathrm{ml}$ ) und/oder Dexamethason (DEX; $1 \mu \mathrm{M}$ ) behandelt wurden.

Ergebnisse. DEX induzierte eine $28,8( \pm 4,5)$-fache SP-B mRNA Hochregulation, während Progesteron die SP-B mRNA Expression hemmte [0,33( $\pm 0,2)$-fold]. In Kombination, konnte die DEX-induzierte Hochregulation von SP-B mRNA durch Progesteron dosisabhängig inhibiert werden. Diese Ergebnisse konnten auf Protein-Ebene mittels WesternBlot-Analyse bestätigt werden.

Diskussion. Progesteron konnte die DEX-induzierte SP-B-Hochregula-

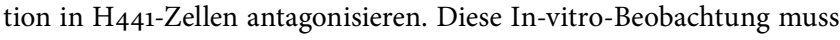
zunächst in vivo bestätigt werden. Bei gleichzeitiger antenataler Steroid- und Progesteron Applikation an die Mutter könnte eine gegensätzliche Wirkungen von Progesteron und Steroiden auf die SurfactantHomeostase von klinischer Relevanz für Frühgeborene sein.

\section{P06-02}

Gibt es eine kurative Behandlungsoption für ein Neugeborenes mit Trachealagenesie?

\section{J. Altstaedt', T. von Kalle'2, Z. el Hafid ${ }^{3}$}

'Olgahospital, Klinikum Stuttgart, Neonatologie, Stuttgart, Deutschland, 2Olgahospital, Klinikum Stuttgart, Radiologie, Stuttgart, Deutschland, ${ }^{3}$ Klinikum Stuttgart, Olgahospital, Stuttgart, Deutschland

Fragestellung. Operationen von Trachealstenosen durch Interponate und Resektionen sind heute auch schon im Neugeborenenalter möglich. Die langstreckige Trachealagenesie jedoch gilt als nicht befriedigend behandelbar. Anhand einer aktuellen Patientin haben wir die Möglichkeiten einer operativen Behandlung einer langstreckigen Trachealagenesie geprüft.

Kasuistik. Primäre Sectio mit $33+5$ SSW bei steigenden Entzündungswerten der Mutter unklarer Genese und massivem Polyhydramnion mit dringendem Verdacht auf Ösophagusatresie. Postnatal frustrane Atemanstrengungen des Kindes bei gutem Atemantrieb, keine Stimme. Unter Maskenbeatmung nur kurzfristige Stabilisierung, bei anhaltender Bradykardie Entschluss zur Intubation, Stimmritze jedoch auch mit Führungsdraht nicht passierbar. Unter medikamentöser Reanimation mit Suprarenin und erneuter Maskenbeatmung gelingt die Kreislaufstabilisierung. Bei unterstellter Trachealagenesie mit ösophagealer Fistel Intubation des oberen Ösophagus, hierunter stabile Ventilation. Durch maschinelle Atemunterstützung mittels SIMV-Beatmung über einen geblockten Tubus im Ösophagus konnte unsere Patientin stabil über die ösophagotracheale Fistel ventiliert und oxygeniert werden, um bildgebende Diagnostik durchzuführen. Das Röntgenbild des Thorax war nicht wegweisend. Im MRT zeigte sich eine langstreckige Trachealagenesie von Kehlkopfebene bis zur Carina (Typ 2 nach Floyd), kombiniert mit einer Ösophagusatresie Typ IIIc nach Vogt, sowie als 
Nebenbefund eine partielle Lungenvenenfehlmündung und ein Ventrikelseptumdefekt; unauffällige Chromosomenanalyse.

Fazit. Obwohl initial aufgrund der Kombination der Fehlbildungen eine Stabilisierung gelungen war, bestand wegen der Länge der Trachealagenesie bei vollständig fehlender Knorpelanlage sowohl nach allen bisher in der Literatur bekannten ähnlichen Fällen als auch nach Expertenmeinung keine kurative Behandlungsaussicht. Im Einvernehmen mit den Eltern wurde daher von einer Intervention abgesehen und das Kind palliativ versorgt. Die Trachealagenesie ist nach wie vor eine sehr schwierig zu therapierende kongenitale Fehlbildung. Eine überregionale interdisziplinäre Vorgehensweise kann bei extrem seltenen Krankheitsbildern helfen, individuelle Entscheidungen im Sinne des Kindes zu treffen.

\section{P06-03 \\ Mittellappenresektion als lebensrettende Maßnahme bei einem extrem kleinen Frühgeborenen mit progredient raumfordern- dem interstitiellem Emphysem}

S. Schüller', C. Lindtner', L. Wisgrill', K. Klebermass-Schrehof', N. Haiden', A. Hojreh $^{2}$, M. Metzelder ${ }^{3}$, A. Berger ${ }^{1}$

'Unversitätsklinik für Kinder- und Jugendheilkunde, MUW, Klinische Abteilung für Neonatologie, Pädiatrische Intensivmedizin und Neuropädiatrie, Wien, Österreich, ${ }^{2}$ Unversitätsklinik für Radiologie und Nuklearmedizin, MUW, Klinische Abteilung für Allgemeine Radiologie und Kinderradiologie, Wien, Österreich, ${ }^{3}$ Unversitätsklinik für Chirurgie, MUW, Klinische Abteilung für Kinderchirurgie, Wien, Österreich

Einleitung. Das pulmonale interstitielle Emphysem ist eine schwerwiegende Komplikation des neonatalen Atemnotsyndroms und gilt als Risikofaktor für das Auftreten einer Hirnblutung oder chronischer Lungenerkrankung. Therapeutische Maßnahmen beinhalten die Hochfrequenzoszillationsbeatmung, sowie bei der unilateralen Form, die selektive Beatmung der kontralateralen Lunge. Die operative Resektion der befallenen Lungenabschnitte ist Ausnahmeverläufen vorbehalten. Fallbericht. Wir berichten über den Fall eines extrem unreifen Frühgeborenen, das aufgrund eines unilateralen pulmonalen interstitiellen Emphysems mit Ausbildung verdrängender Riesenbullae zunächst konservativ therapiert wurde, bei zunehmender klinischer Instabilität schließlich durch eine operative Mittellappenresektion erfolgreich behandelt werden konnte. Ein eutrophes männliches Frühgeborenes der SSW $23+5$ wurde nach vorzeitigem Blasensprung bei unhemmbaren Wehen nach Lungenreifebehandlung per Sectio geboren. Bei deutlichen RDS-Zeichen wurde zunächst Surfactant über eine intratracheal liegende Sonde am spontan atmenden Kind verabreicht. Am 5. Lebenstag erfolgte bei steigendem $\mathrm{O}_{2}$-Bedarf am Infant Flow und zunehmenden Apnoen die Intubation. Radiologisch ließ sich ein HMD I ${ }^{\circ}$ bei sonst unauffälligem Befund nachweisen. Nach zunächst erfolgreicher Extubation musste der Patient am 12. Lebenstag im Rahmen einer Pneumonie (mit Nachweis von Staphylococcus aureus im Trachealsekret) reintubiert werden. An der konventionellen Beatmung kam es zu einer unzureichenden $\mathrm{CO}_{2}$-Elimination, sodass eine Hochfrequenzbeatmung (HFOV) begonnen wurde. Radiologisch fanden sich zunächst kleinzystische Veränderungen im Bereich des Mittellappens mit Verdrängung der restlichen rechten Lunge. Trotz erneuter Extubation und konservativen Maßnahmen (strenge Seitenlagerung, physiotherapeutisches Taping, Vermeidung erneuter mechanischer Beatmung) kam es im Verlauf durch eine sukzessive Volumenzunahme der emphysematösen Veränderungen zur fortschreitenden Kompression der restlichen Lungenabschnitte. In der Folge entwickelte sich nach insgesamt sieben Wochen konservativer Therapie eine schwere pulmonale Hypertonie und letztlich respiratorischer Insuffizienz. Bei dann neuerlich notwendiger mechanischer Beatmung kam es auch zu einer zunehmenden hämodynyamische Instabilität durch kardiale Kompression. Daher entschloss sich das betreuende Team von Neonatologie und Kinderchirurgie zu einem operativen Therapieansatz. Am 82. Lebenstag erfolgte eine Mit- tellappenresektion, welche peri- und postoperativ problemlos verlief. Der Beatmungsaufwand sank bereits unmittelbar postoperativ. Eine Woche später konnte der Patient extubiert werden, erhielt für eine weitere Woche die O2-Brille und war im Anschluss spontan atmend ohne Atemunterstützung. Der Patient konnte dann am errechneten Geburtstermin in sehr gutem Allgemeinzustand von der Neonatologie entlassen werden.

Schlussfolgerung. Das pulmonale interstitielle Emphysem mit Bildung bullöser Veränderungen kann sich beim extrem unreifen Frühgeborenen als Komplikation des neonatalen Atemnotsyndroms, nach Pneumonie sowie nach maschineller Beatmung entwickeln. Bei zunehmender klinischer Instabilität und kontinuierlich hohem Sauerstoffbedarf ist, nach Abwägung von Risiko und Nutzen ein chirurgischer Zugang zu erwägen.

\section{P06-04}

\section{Frühgeborenes mit wiederholten frustranen Extubationsversu- chen und Aspirationspneumonien}

V. Lieftüchter', S. Herber-Jonat', K. Förster', A. Schulze', I. Rost² , W. Müller-Felber $^{3}$, A. Flemmer ${ }^{1}$

'Klinikum der Universität München, Marchioninistraße ${ }^{15},{ }^{81377}$ München, Neonatologie der Kinderklinik am Perinatalzentrum Großhadern, München, Deutschland, ${ }^{2}$ Zentrum für Humangenetik und Laboratoriumsdiagnostik (MVZ), Dr. Klein, Dr. Rost und Kollegen, Martinsried, Deutschland, ${ }^{3}$ Dr. von Haunersches Kinderspital, Abteilung für Neuropädiatrie, München, Deutschland

Hintergrund. Bei sehr unreifen Frühgeborenen wird versucht, die Zeit an der invasiven Beatmung zu verkürzen oder diese ganz zu vermeiden. Wenn aber Extubationen immer wieder frustran verlaufen, muss eine systemische Grunderkrankung in die Differentialdiagnosen mit einbezogen werden.

Kasuistik. Wir berichten über ein Frühgeborenes der 27 4/7 SSW nach Entbindung durch primäre Sectio bei HELLP der Mutter. Nach Erstversorgung mit Intubation und Surfactantgabe bei initial ausgeprägtem RDS wurde der respiratorische Verlauf durch rezidivierende Aspirationspneumonien und mehrere frustrane Extubationsversuche kompliziert. Hinweise für eine laryngeale Spalte ergaben sich in der Bronchoskopie nicht. Klinisch zeigte das Frühgeborene nur wenig Spontanmotorik, eine ausgeprägte Spitzfußstellung mit muskulärer Hypertonie der distalen unteren Extremität, sowie sonographisch und radiologisch ein Zwerchfellhochstand rechts bei atemsynchroner Beweglichkeit des Zwerchfells. Phänotypisch waren, keine weiteren syndromalen Stigmata vorhanden. In der Anamnese der Mutter fielen rezidivierende Herzrhythmusstörungen auf. Klinisch zeigte sie eine $\mathrm{Fa}$ cies myopathica, eine verzögerte Muskelerschlaffung nach Händedruck und ein schleppendes Gangbild.

Laboruntersuchungen. Aufgrund des klinischen Bildes eines „floppy infant" und der mütterlichen Symptomatik veranlassten wir eine molekulargenetische Untersuchung auf Trinukleotid-Repeat-Expansion. Im DMPK-Gen wurde auf einem Allel eine pathologische CTG-Triplett-Expansion um 2500-2700 Kopien durch Southern Blot-Analyse nachgewiesen. Damit konnte die Verdachtsdiagnose einer myotonen Dystrophie Typ-1 bestätigt werden.

Schlussfolgerung. Die myotone Dystrophie ist eine autosomal-dominant vererbte Multisystemerkrankung, die bei Neugeborenen vor allem durch eine Muskelschwäche und dadurch, durch ein postnatales respiratorisches Versagen gekennzeichnet sein kann. Die myotone Dystrophie Typ 1 (Curschmann-Steinert) ist mit einer Inzidenz von 1:80oo die häufigste muskuläre Dystrophie im Erwachsenenalter. Unser Fall illustriert, dass diese Erkrankung bei Frühgeborenen mit rezidivierenden Aspirationen und frustranen Extubationsversuchen in die Differentialdiagnosen mit einbezogen werden sollte. 
P06-05

\section{Anwendung von nasalem HFOV bei Frühgeborenen: Ergebnisse einer retrospektiven Analyse}

\section{Stavropoulou', D. Klotz', H. Fuchs', R. Hentschel' \\ 'Universitätsklinikum Freiburg, Zentrum für Kinder- und Jugendmedizin, Neonatologie/Intensivmedizin, Freiburg, Deutschland}

Hintergrund. Die Vorteile der nichtinvasiven Atemunterstützung, verglichen mit der invasiven Beatmung sind in mehreren Publikationen beschrieben. Die nasale Hochfrequenzoszillation (nHFOV) stellt eine neue Form der nichtinvasiven Atemunterstützung dar, ihr wird eine effektivere $\mathrm{CO}_{2}$-Elimination und eine Vermeidung invasiver Beatmung zugeschrieben.

Fragestellung. Stellt nHFOV, verglichen mit nasalem CPAP, bei sehr kleinen Frühgeborenen eine sichere Alternative der Atemunterstützung dar? Ist die $\mathrm{CO}_{2}$-Elimination verbessert, und können dadurch Intubationen vermieden werden.

Material und Methode. Retrospektive Analyse des Verlaufs und Outcomes von 24 Frühgeborenen $<34$ SSW, die zwischen 3/2013 und 12/2014 mit nHFOV behandelt wurden.

Ergebnisse. 11 von 24 Frühgeborenen mit einem medianen Gestationsalter von $29+6 / 7$ (Spannweite 24-34) SSW wurden bei einem $\mathrm{pCO}_{2}$ von $67(83-40) \mathrm{mmHg}$ von nCPAP auf nHFOV umgestellt. Nach $2-4 \mathrm{~h}$ fie das $\mathrm{pCO}_{2}$ auf $56(40-60) \mathrm{mmHg}$ und nach $48 \mathrm{~h}$ auf $44(39-49) \mathrm{mmHg}$. Der Sauerstoffbedarf wurde unter nCPAP bei 23\% (21-30) gemessen und unter nHFOV ebenfalls bei $23 \%$ (21-28). 12 von 24 Frühgeborenen mit einem GA von 25+6/7 (22-30) SSW wurden direkt nach Extubation auf nHFOV umgestellt. Der $\mathrm{pCO}_{2}$ betrug vor Extubation $53(43-73) \mathrm{mmHg}$ Nach 2-4 h fiel das pCO2 auf 52 (41-72) mmHg und nach $48 \mathrm{~h}$ auf 45 (41-61)mmHg. Der Sauerstoffbedarf unter der Beatmung zählte $28 \%$ (21-45) und nach Extubation 30\% (21-45). Nur 1 von 12 Kindern aus der Extubationsgruppe musste innerhalb von 48 Stunden aus nHFOV aufgrund von Apnoen und Bradykardien invasiv beatmet werden. 1 Kind wurde primär nach der Erstversorgung mit nHFOV behandelt und wurde nach 10 Stunden intubiert. Es wurden keine Nebenwirkungen der Behandlung mit nHFOV, wie Pneumothorax, starke abdominelle Distension, Nahrungsunverträglichkeit, vermehrte Unruhe oder ähnliches beobachtet.

Diskussion. In dieser Kohorte war die $\mathrm{CO}_{2}$-Elimination, wie in anderen publizierten Kohorten, mit nHFOV bei nicht-invasiv beatmeten Frühgeborenen effektiv. Die Oxygenierung scheint nicht in gleichem Maße positiv beeinflussbar zu sein.

Schlussfolgerung. Um das geeignete Patientenkollektiv, Wirkmechanismen und optimale Einstellung bei der nHFOV-Beatmung zu identifizieren sind weitere randomisierte Studien erforderlich.

\section{P06-06 \\ Leckage verbessert die $\mathrm{CO}$-Elimination bei nichtinvasiver nasa- ler Hochfrequenzoszillations-Beatmung}

D. Klotz', C. Schäfer', D. Stavropoulou', S. Schumann², H. Fuchs ${ }^{1}$

'Universitätsklinikum - Zentrum für Kinder- und Jugendmedizin, Abteilung für Neonatologie und Pädiatrische Intensivmedizin, Freiburg, Deutschland, ${ }^{2}$ Klinik für Anästhesiologie und Intensivmedizin, Experimentelle Anästhesiologie, Freiburg, Deutschland

Hintergrund. Die nasale Hochfrequenzoszillation (nHFOV) ist eine neuere Form der Atemunterstützung in der Neonatologie. Über einen kontinuierlichen Gasfluss wird ein konstanter mittlerer Atemwegsdruck (MAP) aufrechterhalten, auf den eine oszillatorische Druckkurve appliziert wird. Klinische Fallserien, Observations- und Modellstudien zeigen eine signifikant bessere $\mathrm{CO}_{2}$-Elimination durch nHFOV im Vergleich zu nasalem CPAP. Ein bisher nicht untersuchter Aspekt ist der Einfluss des im Rahmen der nicht-invasiven Beatmung entstehenden Leckflusses auf die Effektivität des Gasaustausches. Wir untersuchten den tatsächlichen Einfluss eines kombinierten nasalen und oralen
Leckageflusses auf die $\mathrm{CO}_{2}$-Auswaschung unter nHFOV an einem physikalischen Lungenmodell des neonatalen respiratorischen Systems. Methoden. Ein konventioneller Respirator wurde mittels nasaler Prongs mit einem neonatalen Atemwegs- und Lungenmodell verbunden, welches bzgl. Totraum, Volumen,Resistance und Compliance einem Frühgeborenen mit Atemnotsyndrom glich. Mit Hilfe einer kontinuierlichen $\mathrm{CO}_{2}-\mathrm{Zufuhr}$ in die künstliche Lunge wurde eine konstante alveoläre $\mathrm{CO}_{2}$-Konzentration eingestellt, eine verstellbare Apparatur erlaubte es, im Äquivalent des Oropharynx eine definierte Leckage einzustellen. Unter variierenden Frequenzen, Amplituden und Leckstufen wurde simultan kontinuierlich die alveoläre $\mathrm{CO}_{2}$-Clearence bestimmt, sowie Druck-, bzw. Flussmessungen in der Leckage, pulmonal und nahe der Prongs durchgeführt und mittels einer eigens entwickelten Software aufgezeichnet.

Ergebnisse. Das beschriebene Modell lieferte valide und replizierbare Messungen. Bei maximal geöffnetem Leck unter nHFOV war keine effektive $\mathrm{CO}_{2}$-Elimination messbar. Ohne Leckfluss ging die Erhöhung der Amplitude, bzw. eine Reduktion der Frequenz, erwartungsgemäß mit einer Zunahme der $\mathrm{CO}_{2}$-Elimination einher. Bei mittlerem Leckfluss hingegen zeigte sich eine signifikant effektivere $\mathrm{CO}_{2}$-Elimination im Vergleich zur Ventilation ohne Leckage. Dieser Effekt ließ sich bei jeder verwendeten Amplitude und Frequenz reproduzieren ( $\mathrm{p}<0,001)$. Diskussion. Dies ist die erste beschriebene Untersuchung über den Einfluss einer Leckage auf die $\mathrm{CO}_{2}$-Elimination unter nichtinvasiver Beatmung. Wir konnten am ersten beschriebenen neonatalen Lungenmodell mit kontinuierlicher $\mathrm{CO}_{2}-\mathrm{Zufuhr}$ zeigen, dass eine Leckage unter nHFOV die $\mathrm{CO}_{2}$-Elimination verbessern kann. In der klinischen Praxis wird bei nichtinvasiver Beatmung versucht, eine Leckage durch verschiedene Maßnahmen (Lagerung, etc.) gering zu halten, um ein angestrebtes positives endexpiratorisches Druckniveau zu erreichen. Inwieweit nichtinvasiv beatmete neonatologische Patienten mit $\mathrm{CO}_{2}$ Retention von einem Leck profitieren können, ist bisher nicht untersucht. Unklar bleibt der Einfluss der Spontanatmung, dies ist Gegenstand unserer laufenden Untersuchungen.

\section{P06-07}

Messung generierter Spitzendrücke beim Gebrauch von High-flow-nasal-cannula-Geräten - eine In-vitro-Studie

\section{S. Dold', H. Hummler', M. Mendler}

${ }^{1}$ Klinik für Kinder- und Jugendmedizin, Universitätsklinikum Ulm, Sektion Neonatologie und Pädiatrische Intensivmedizin, Ulm, Deutschland

Hintergrund. Binasale High-Flow-Systeme sind eine zunehmend weltweit beliebte Form der nichtinvasiven Atemunterstützung bei Neonaten. Die Definition bezüglich des „high“ Flows variieren. Generell spricht man ab Flowraten $>1$ Liter/Minute von High-flow. Die gängigen High-flow-Systeme erwärmen und befeuchten das Atemgas und sind mit einem Sauerstoffmischer versehen. Diese Systeme werden als primäre Form der Atemunterstützung beim Atemnotsyndrom, zur Atemunterstützung nach Extubation, zur Therapie von Apnoen, sowie zur Entwöhnung von kontinuierlicher positiver Druckunterstützung (NCPAP) verwendet. Traditionell wurden High-flow-Systeme in der Therapie älterer Kinder eingesetzt, vor allem bei viralen Atemwegsinfektionen.

Fragestellung. Ziel der vorliegenden Arbeit ist es die Funktion und die Sicherheit verschiedener High-Flow Systeme am Modell zu untersuchen. Insbesondere wurde der maximale Druck bei Komplettokklusion an verschiedenen Stellen der Atemwege gemessen.

Material und Methoden. In einem in-vitro Modellversuch untersuchten wir die generierten Spitzendrücke unter Verwendung verschiedener Flowraten und Nasenbrillengrößen bzw. am intubierten Modell mit zwei verschiedenen High-Flow Systemen (Vapotherm \& Optiflow). Bei dem Modell handelte es sich um ein Neugeborenenmodell der Firma Laerdal, an welches zwei Testlungen der Firma Dräger angeschlossen wurden. Die Messungen erfolgten an 3 Punkten im Modell (proximal und distal eines definierten Lecks, sowie in der Testlunge) um den ma- 


\begin{tabular}{|c|c|c|c|c|c|c|}
\hline & Vor Leck $\left(\mathrm{cmH}_{2} \mathrm{O}\right)$ & Nach Leck $\left(\mathrm{cmH}_{2} \mathrm{O}\right)$ & Lunge $\left(\mathrm{cmH}_{2} \mathrm{O}\right)$ & Vor Leck (okk) $\left(\mathrm{cmH}_{2} \mathrm{O}\right)$ & Nach Lunge (okk) $\left(\mathrm{cmH}_{2} \mathrm{O}\right)$ & Lunge (okk) $\left(\mathrm{cmH}_{2} \mathrm{O}\right)$ \\
\hline 15I Vapotherm & 76 & 80 & 77 & 457 & 482 & 455 \\
\hline 6I Vapotherm & 16 & 17 & 16 & 265 & 274 & 263 \\
\hline 15I Optiflow & 19 & 22 & 20 & 49 & 49 & 28 \\
\hline $\begin{array}{l}61 \\
\text { Optiflow }\end{array}$ & 13 & 15 & 14 & 38 & 39 & 39 \\
\hline
\end{tabular}

ximalen Druck in den Atemwegen bei Komplettokklusion zu simulieren.

Ergebnisse. Wir fanden deutliche Unterschiede zwischen den beiden Geräten. Die Sicherheitsmechanismen sind offenbar gerätespezifisch und scheinen im niedrigeren Flowbereich besser zu greifen. Mit höherem Flow können sehr hohe Drucke bei Komplettokklusion auftreten (Tab. Po6-07).

Schlussfolgerung. Insbesondere unter Verwendung hoher Flowraten, sollte auf die korrekte Wahl der Nasenbrillengröße und ein ausreichend großes Leck geachtet werden. Eine Komplettokklusion ist vermutlich im klinischen Alltag sehr selten, kann aber zu relevanten Druckanstiegen im Bereich der Atemwege führen obwohl das Überdruckventil des Systems geöffnet wird.

\section{P06-08}

\section{Quantitativer Vergleich neonatologischer Respiratoren}

\section{T. Krieger', M. Wald}

'Universitätsklinik für Kinder- und Jugendheilkunde Salzburg, Division für Neonatologie, Salzburg, Österreich, ${ }^{2}$ Salzburger Universitätsklinikum, Neonatologie, Salzburg, Österreich

Hintergrund. Klinische Anwender können aus einem breiten Angebot an neonatologischen Respiratoren wählen. Viele Hersteller verwenden unterschiedliche Begriffe, um identische Beatmungsformen zu bezeichnen. Andere wiederum setzen verschiedene Sensortechnologien, Software-Strategien und Kalibrationsumgebungen ein, versprechen aber die gleiche Performance. Vor dem Hintergrund eines technologisch, terminologisch und algorithmisch gesättigten Marktes zielt diese vergleichende Anwenderstudie darauf ab, die relative Sensor- und Steuerungs-Performance von sechs gebräuchlichen Respiratoren aufzuzeigen.

Methoden. Die Atmungseigenschaften typischer neonataler Patienten wurden auf einem mechanischen, neonatalen aktiven Lungenmodell (NALM, Dr. Schaller Medizintechnik) simuliert. Hiermit konnten präzise reproduzierbare Beatmungsbedingungen für die Untersuchung von sechs neonatologischen Respiratoren (Babylog 8oooplus, Fabian, Leoni Plus, Servo-N, Sophie, Stephanie) gewährleistet werden. Auf allen Beatmungsmaschinen wurden die gleichen Beatmungsmodi eingestellt. Erhoben wurde sowohl die relative Übereinstimmung im Erreichen der Zielwerte (Tidalvolumen, Atemfrequenz etc.), als auch die Güte der Adaptation an irreguläre und apnoeische Atemmuster.

Ergebnisse. Unter der Zielvorgabe, ein Tidalvolumen von 21,00 ml zu garantieren, lag das größte erreichte mittlere Tidalvolumen relativ zum Referenzsensor 4,03 $\mathrm{ml}(21,4 \%)$ über dem kleinsten: Stephanie $(18,81 \pm 1,28 \mathrm{ml})$, Servo-N $(18,96 \pm 1,17 \mathrm{ml})$, Leoni Plus $(20,83 \pm 0,58 \mathrm{ml})$, Sophie $(21,02 \pm 0,64 \mathrm{ml})$, Babylog 8oooplus $(22,50 \pm 0,43 \mathrm{ml})$, Fabian $(22,84 \pm 1,19 \mathrm{ml})$. Eine Varianz der gleichen Größenordnung wurde für alle Zielvolumina gemessen.

Schlussfolgerung. Klinische Anwender sollten sich insbesondere bei einem Wechsel von Geräten der unterschiedlichen Respiratorenperformance bewusst sein. Die beobachtete Abweichung der produzierten Tidalvolumina betrug bis zu $21 \%$ und muss als klinisch relevant eingestuft werden. Diese Variabilität nicht zu berücksichtigen könnte schlimmstenfalls zum Setzen eines Volutraumas führen.

\section{P06-09}

Regulation von proinflammatorischen PACAP-Rezeptoren beim Respiratory-Distress-Syndrom (RDS) des Früh- und Neugeborenen

M. Müller', A. Kislat', A. Hippe', A. Poppe', S. Goldmann', T. Paul', S. Seeliger'

'Universitätsklinikum Göttingen, Abteilung für Pädiatrische Kardiologie, Intensivmedizin und Neonatologie, Göttingen, Deutschland, ${ }^{2}$ Heinrich Heine Universität, Klinik und Poliklinik für Dermatologie, Düsseldorf, Deutschland

Einleitung. Die Prävalenz des Respiratory-Distress-Syndroms (RDS) liegt bei Frühgeborenen unter 32 Schwangerschaftswochen (SSW) bei ca. 50\%. Ursache ist ein primärer Surfactantmangel. In Folge kommt es zu einer erhöhten alveolären Oberflächenspannung, zu Atelektasen/ Dystelektasen und zu einer Entzündungsreaktion der neonatalen Lunge, der wichtigste Faktor für die Entwicklung einer bronchopulmonalen Dysplasie (BPD), die 8\% der Kinder $<32$ SSW entwickeln. Als die effektivste Therapie gegen das neonatale RDS gilt die Gabe von exogenem Surfactant. Die Frage, ob exogenes Surfactant inflammatorische Prozesse triggert ist zunehmend in Vergessenheit geraten. Der Verbrauch von exogenem Surfactant hat aber gerade bei Nutzung wenig invasiver Applikationsformen (LISA) um bis zu 30\% zugenommen. In der Literatur gibt es Anhaltspunkte, dass auch Pituitary adenylate cyclase-activating polypeptide (PACAP) und seine Rezeptoren (VPAC1, VPAC2, $\mathrm{PAC} 1)$ inflammatorische, wie auch antiinflammatorische Prozesse beeinflussen. Ob die in Deutschland kommerziell erhältlichen Surfactantpräparationen eine Entzündungsreaktion im neonatalen Lungengewebe beeinflussen und eine Regulation der Entzündungsreaktion auch über PACAP erfolgt wurde in vivo und auf PBMCs ex vivo untersucht. Methode und Ergebnisse. Mittels FACS- und ELISA-Verfahren konnte erstmalig auf mit Lipopolysaccharid (LPS) aus E. coli vorstimulierten Zellen des Blutes (PBMC) gezeigt werden, dass Surfactantpräparationen zu einer deutlichen Hochregulation des pro-inflammatorischen Interleukin (IL)-8 führten. Parallel fand sich auf Zellen neonataler Trachealsekrete (Cystospinpräparate) mit Surfactant behandelter Frühund Neugeborener eine Hochregulation des proinflammatorischen Rezeptors VPAC1, was über Adhäsionsmoleküle Entzündungsprozesse unterhält.

Schlussfolgerung. Die aufsteigende Infektion der Schwangeren, die infektiöse Infiltration der Eihäute wie sie bei einem Amnioninfektionssyndrom (AIS) zu finden ist, wird als die häufigste Ursache einer Frühgeburt beschrieben. Viele dieser Kinder benötigen im klinischen Alltag aufgrund eines RDS exogenes Surfactant. Wir konnten in vitro zeigen, dass nach Vorstimulation von PBMCs und anschließender Surfactantgabe das pro-inflammatorische Signal verstärkt wird. Dies konnte an Zellen aus Trachealsekreten von Früh- und Neugeborenen bestätigt werden. Diese Befunde sollten im klinischen Alltag Anlass zur kritischen Indikationsstellung einer Surfactantapplikation geben, da Surfactant möglicherweise eine pulmonale Entzündungsreaktion triggert, und so an der Entwicklung einer bronchopulmonalen Dysplasie mitbeteiligt sein kann. 
P06-10

\section{Unterdrucklungenödem - unterdiagnostizierte Ursache für} Sauerstoffbedarf bei Kindern?

\section{Thiel', J. Paulsen', S. Mayer', C. Rasch'²}

'Sana-Klinikum Remscheid, Klinik für Kinder und Jugendliche, Remscheid, Deutschland, ${ }^{2}$ Sana-Klinikum Remscheid, Zentrum für Anästhesie, Intensiv-, Schmerz- und Palliativmedizin, Remscheid, Deutschland

Hintergrund. Passagerer Postoperativer Sauerstoffbedarf ist ein häufiges Phänomen im Kindesalter. Wir berichten über eine Ursache, die dem Kinderanästhesisten geläufiger ist als dem Pädiater.

Kasuistik. 15-jähriger Junge mit laparoskopischer Appendektomie bei phlegmonöser Appendizitis. Bei Ausleitung $\mathrm{SaO}_{2}$-Abfall nach Tubusbiss, kein Hinweis auf Aspirat oder Mageninhalt beim Absaugen, keine vorbestehende Infektion oder Atemwegsaffektion, keine Volumenüberladung oder Medikamenteninduktion. $\mathrm{SpO}_{2} 70 \%$ bei $151 \mathrm{O}_{2}$, Giemen, grobblasige RG. CPAP/ASB mit $\mathrm{FiO}_{2} \mathrm{o}, 5$, PEEP $10 \mathrm{cmH} 2 \mathrm{O}$. Radiologisch bilaterale teilweise konfluierende, flaue Verdichtungen. BGA ausgeglichen, Labor normal. Therapie: CPAP, O2. Am Folgetag Normalisierung, unauffälliges Thorax-Röntgenbild.

Diskussion. Ein Unterdrucklungenödem ist eine Komplikation akuter und chronischer Obstruktion der oberen Atemwege. Vorherrschender pathophysiologischer Mechanismus: Entwicklung eines ausgeprägten, negativen (niedriger als Umgebungsdruck) intrapleuralen Druckes während der Inspiration gegen die geschlossene Glottis (Inspiration normal: -7 bis $-8 \mathrm{cmH}_{2} \mathrm{O}$, bei Glottisverschluss: $-5 \mathrm{O}$ bis $-140 \mathrm{cmH}_{2} \mathrm{O}$ ). Dadurch erhöhter pulmonaler mikrovaskulärer Druck führt zu Flüssigkeitsübertritt in den peribronchialen und perivaskulären Raum. Die pleurale Druckverringerung bewirkt zudem eine Erhöhung des venösen Rückflusses zum rechten Herzen, während die linksventrikuläre Nachlast steigt. Erstbeschreibung beim Kind 1973, beim Erwachsenen 1977. Typ I: durch z. B. Laryngospasmus, Biss auf Tubus oder Larynxmaske oder Epiglottitis. Typ II: nach operativer Entlastung chronisch obstruierter Atemwege vor, z. B. bei chronischer Tonsillitis oder Adenoidhypertrophie. Klinik: Tachydyspnoe, inspiratorischen Stridor, Cyanose, schaumiges, hämorrhagisches Sekret, Unruhe, Agitiertheit. DD v.a. Aspiration oder Anaphylaxie. Therapie abhängig vom Schweregrad Sauerstoff, antiobstruktive Inhalationen, Beatmung mit PEEP, meistens selbstbegrenzt mit supportiver Therapie, Flüssigkeitsrestriktion und Diuretika nicht notwendig. Prophylaxe: Gute Vorbereitung der Narkoseausleitung, frühzeitiger Behebung akuter oder chronischer Atemwegsobstruktion. Trotz hoher Inzidenz von Laryngospasmen im Kindesalter postobstruktives Lungenödem selten. Der Grund ist unklar (extrem elastische Thoraxwände, Aufbau hoher negativer intrapleuraler Drücke möglich. Epiglottitis und Laryngotracheobronchitis sind zu über $50 \%$ an Fällen von Lungenödemen bei Kindern unter 10 Jahre beteiligt). Unterdrucklungenödem ist mögliche Ursache für anders nicht hinreichend erklärbaren Sauerstoffbedarf, z. B. bei Laryngitis subglottica nach Steroid/Adrenalin-Behandlung oder Fremkörperaspiration oder milden, anders nicht erklärten Sauerstoffbedarf nach Beatmung. Inzidenz 0,05-0,1\%, wahrscheinlich höher (Verschleierung durch die Aspiration von Mageninhalt, Auslöser und Klinik ähnlich; Häufigkeit oberer Atemwegsobstruktionen in der perianästhetischen Phase; falsch diagnostizierte Aspiration; manifeste Klinik in der Regel nach Beseitigung der oberen Atemwegsobstruktion oder verzögert, Zeitspanne 3 bis 150 Minuten bis zu mehreren Tagen; bei gering ausgeprägter Symptomatik unerkannt).

Schlussfolgerung. Die Kenntnis der oft harmlosen Ursache kann zur Beruhigung beitragen und das Kind vor nicht notwendiger Diagnostik und Therapie bewahren.

\section{Posterwalk 7 - Entwicklungsfördernde Pflege}

\author{
P07-01 \\ Spirituelle Bedürfnisse von Müttern von Früh- und kranken Neu- \\ geborenen \\ U. Waßermann', M. Thiel', A. Büssing ${ }^{3}$ \\ 'Gemeinschaftskrankenhaus Herdecke, Kinder- und Jugendpsychiatrie, \\ Herdecke, Deutschland, ${ }^{2}$ Sana-Klinikum Remscheid, Klinik für Kinder \\ und Jugendliche, Remscheid, Deutschland, ${ }^{3}$ Professur für Lebensquali- \\ tät, Spiritualität und Coping, Institut für Integrative Medizin, Herdecke, \\ Deutschland
}

Hintergrund. Spiritualität ist Teil basaler menschlicher Bedürfnisse. Spiritualität als Ressource zur Bewältigung schwerwiegender Lebensereignisse wird in Kliniken häufig ignoriert, da es außerhalb der professionellen Pflichten von Ärzten, Pflegenden und Psychologen liegt. Inzwischen gibt es eine wachsende Evidenz, dass auch innerhalb säkularer Gesellschaften chronisch kranke Patienten spezifische spirituelle Bedürfnisse haben. Bisher ist wenig über diese Bedürfnisse bei Müttern von Frühgeborenen und kranken Neugeborenen bekannt. Ziel der noch laufenden Studie war daher diese Bedürfnisse zu identifizieren. Langfristig sollen die Daten dazu beitragen die Unterstützung der Mütter durch das Personal der Neonatologie zu verbessern.

Methodik. Anonyme Befragung mittels standardisiertem Fragebogen von Müttern Frühgeborener und kranker Neugeborener, mit mindestens 3 Tage dauernden stationären Aufenthalt auf den neonatologischen Stationen in 2 Kliniken. Abhängig von der Schwere der kindlichen Symptomatik, repräsentiert durch die zu erwartende Dauer des stationären Aufenthaltes wurden 2 Gruppen gebildet.(„short term“ vs. „long term“). Folgende standardisierte Fragebögen kamen zur Anwendung: Spiritual needs Questionnaire (SpNQ); Spiritual Wellbeing (FACIT-Sp); Mood states (ASTS); Stress perception (Cohen's PSS); Life Satisfaction (BMLSS-10); Self Efficacy Expectation (Schwarzer's SEE).

Ergebnisse und Diskussion. Bisher wurden 106 Mütter mit einem mittleren Alter von $31 \pm 5$ Jahren eingeschlossen. 97\% lebten mit einem Partner; $67 \%$ hatten Abitur, $75 \%$ gehörten einer christlichen Religion an (4\% andere, 21\% keine Religionszugehörigkeit.); 38\% „short term“, 52\% „long term“. Psychosoziale und spirituelle Bedürfnisse. Die stärksten Bedürfnisse bestanden nach „Innerem Frieden“ und „Unterstützung durch Partner und Familie“. Daneben war es auch wichtig, dem Leben einen Sinn zu geben und zu beten (37\%). Bisher zeigen die Ergebnisse keine relevanten Unterschiede zwischen den Gruppen „short term“ vs. „long term“. Empfinden von Stress und Lebenszufriedenheit. Die Mütter beider Gruppen empfanden eine Beeinträchtigung des Alltagslebens $(56 \pm 25$ vs. $60 \pm 21$; n.s.), das Gefühl unter Druck zu stehen $(50 \pm 29$ vs. $57 \pm 24$; n.s.) bei gleichzeitig relativ hoher Lebensqualität $(80 \pm 15$ vs. $80 \pm 12 ;$ n.s). Die Dimensionen mit niedriger mit niedriger Lebenszufriedenheit wurden durch den Gesundheitszustand des Kindes, die eigene gesundheitliche Verfassung und die Fähigkeit den Alltag zu meistern bestimmt.

Korrelationsanalyse. Insbesondere das Bedürfnis nach „Innerem Frieden" war mit höherer Stressempfindung und stärkerer Beeinträchtigung des Alltagslebens assoziiert.

Schlussfolgerungen. Mütter von Frühgeborenen und kranken Neugeborenen erleben Stress und Alltagsbeeinträchtigungen. Unterstützung erfahren sie durch das neonatologische Team und durch ihren Partner. Es bestehen Bedürfnisse nach Innerem Frieden und Beziehung. Mütter Frühgeborener und kranker Neugeborener haben Bedarf an zusätzlicher Unterstützung in der durch hohe Unsicherheit gekennzeichneten Postnatalphase. Wir hoffen mit diesen Ergebnisse einen Beitrag zur Verbesserung des Coping von Müttern von Frühgeborenen und kranken Neugeborenen zu liefern. 
Tab. P07-02

Measures, parameters and outcomes

\begin{tabular}{|llll}
\hline Patient-related & Healthcare $p$ & Process of care & Administrative \\
\hline Sepsis & Bedside round length & Medical errors & Equipment breakdown \\
\hline Short-term & Stress and satisfaction & Vascular complication & NICU stay length \\
\hline Long-term & Nursing workload & Resources allocation & Bed occupancy \\
\hline $\begin{array}{l}\text { Stress and satisfaction of parents and } \\
\text { patient }\end{array}$ & & Noise level assessment & Average cost per patient \\
\cline { 2 - 4 } & & Intra-unit baby transfers & Overtime payment and sickness days \\
\hline
\end{tabular}

\section{P07-02}

Effect of cohorting patients by level of acuity to designated areas within a neonatal intensive care unit - description of the Microsystems project

\section{A. Bakry', S. Dutta', S. El-Helou', G. Fusch', L. Aliberti', C. Meyer', C. Fusch' 'Department of Pediatrics, McMaster University, Hamilton, Kanada}

Background. There are different organizational models to provide care in NICUs; however, there is no or limited data on the effect of cohorting vs. mixed acuity model. Our plan to switch from the mixed acuity model to cohorting/Microsystems model creates a unique opportunity to add some evidence by introducing it as a research project.

Objective. To measure the effectiveness, efficacy, and the impact of the introduction of Microsystems in NICU, using various domains.

Methods. On May 1, 2014, Microsystems based management was implemented at McMaster University NICU; a 47-bed level III unit with approx. 1000 admissions per year and 300 healthcare providers (HCP). The five-pod NICU was switched from a model of care with mixed acuity to one where babies are allocated according to their acuity of sickness. Before implementation of Microsystems, we had three teams "North", "South" and "Central" with similar mix of acuity, whereas; after the start of Microsystems, teams were differently re-arranged as "Acute-1", "Acute-2" and "Intermediate Care" teams. The newly developed teams comprise physicians, nurses, nurse practitioners, and respiratory therapists. The study duration includes: 1. pre-implementation phase from Jan. 2014 to Apr. 2014, 2. transition phase from May 2014 to Jul. 2014, and 3. post-implementation phase from Aug. 2014 to Jun. 2015. The implementation was prepared over a 12-month period with a weekly meeting of an interdisciplinary group including all HCP. The impact of Microsystems was assessed by 17 sub-projects from 4 domains (patient-, healthcare personnel-, process of care-, and administrative-related; Tab. Po7-02).

Results. Meetings $(\mathrm{n}=40)$ of the interdisciplinary group started in summer 2013. We successfully implemented Microsystems on May 1, 2014. Currently all pre-implementation data are collected according to plan as well as the transition phase data. First results of the subprojects are reported separately (nursing work load, noise and round length).

Conclusion. This unique opportunity will give us the chance to create scientific bases about the effect of different care models in a NICU setting. Not all babies are equally sick within NICU; their level and complexity of sickness vary, and so do their care requirements accordingly. However, the standard model of care has the extremely sick neonates fully interspersed between the less sick ones across the unit, which hinders the development of teams specialized in the care of each patient group.

Acknowledgements. The project is funded by HAHSO. The other members of the working group are: Debborah Barnard, Jennifer Callen, Joanne Doucette, Sourabh Dutta, Donna LaForce, Michael Marrin, Deb Patterson, David Pogorzelski, Karen Prim, Samira Samiee, Karen Schatthauer, Sandesh Shivananda, Sumesh Thomas, Connie Williams.
P07-03

\section{Effect of the "Microsystems" care model on noise reduction in the NICU - first results}

G. Fusch', S. Mohamed', A. Bakry', N. Al Sharari', D. Pogorzelski', C. Meyer', L. Alberti', S. El-Helou', S. Ziada', C. Fusch'

${ }^{1}$ Pediatrics, McMaster University, Hamilton, Kanada, ${ }^{2}$ Mechanical Engineering, McMaster University, Hamilton, Kanada

Background. There are different organisational models to manage a NICU. We recently introduced "Microsystems" and cohorting of patients according to acuity in our level III unit. One outcome parameter to assess the impact of this change is the noise level as this implementation will create designated areas with more and less intensive care within the NICU. We hypothesize that noise levels will be different in both areas before and after introduction.

Objectives. 1) To assess the noise level before and after implementation of Microsystems comparing day vs. night time, weekdays vs. weekends and areas of high and low acuity, 2) to investigate the effect of the implementation of Microsystems on the noise levels in the different areas of the NICU

Methods. Institution: McMaster University NICU, a 47-bed level III; 5 pods with 4 X 10 and $1 \times 7$ beds. Successful implementation of Microsystems on May 1st, 2014 after a preparatory phase of 8 months.

Data collection. Continuous 24-h noise level measurements (6o days before and 50 days after implementation) in two representative pods (pod A: increased acuity, pod D: reduced acuity after implementation) using a commercial available noise meter (Casella, CEL-246). The meter was positioned in the center of each of the five pods and moved to the next pod on a daily schedule. Noise levels were measured as LAeq in dBA in $10 \mathrm{sec}$ intervals and averaged over $5 \mathrm{~min}$. Different times were analyzed, daytime (6am to $9 \mathrm{pm}$ ) and nighttime (9pm to $6 \mathrm{am})$. Time: Data was collected during two periods: 1. pre-implementation phase from March to Apr 2014; 2. post-implementation phase from Sept. to Nov. 2014.

Results. Weekdays: Pre-implementation: Average noise levels in pod $A$ and D were $61 \pm 0.5$ (day) and $57 \pm 0.5 \mathrm{dBA}$ (night). During hand-over time of nurses (morning and evening), average noise levels reach $64 \pm 0.5$ $\mathrm{dBA}$, maximum noise levels were $72 \mathrm{dBA}$. Post-implementation. pod A (acute care area): Average noise levels unchanged (61 $\pm 0.5 \mathrm{dBA}) \mathrm{du}-$ ring the day and elevated $(58 \pm 0.5 \mathrm{dBA})$ during night potentially due to increased need of medical equipment such as ventilators. Noise peaks during hand-over of nurses $(64 \pm 0.5 \mathrm{dBA})$ are similar to before start of microsystems. Pod D (intermediate care area): average noise levels significantly reduced to $58 \pm 0.5$ and $57 \pm 0.5 \mathrm{dBA}$ during day and night, respectively. Noise peaks during hand-over have been reduced to $62 \pm 0.5$ $\mathrm{dBA}$ in the morning or disappeared completely in the evening. Weekends: Compared to weekdays, average noise levels were significantly reduced by at least $3 \mathrm{dBA}$ to $58 \pm 0.5 \mathrm{dBA}$ (day) and $55 \pm 0.5 \mathrm{dBA}$ (night). Conclusion. Measured noise levels are higher than current recommendations for NICUs (AAP: $<45 \mathrm{dBA}$ ). Noise level seems to be more affected by organisational conditions (e.g. handover, day/night and weekends). Overall, MS seems to lower the noise exposition significantly; it does not increase the noise exposition in the acute area but reduces it in the intermediate area.

Acknowledgement. The project is funded by HAHSO. 
P07-04

\section{Introduction of a "Microsystems" care model in the NICU -} impact on round length

D. Pogorzelski', A. Bakry', C. Meyer', L. Alberti', S. El-Helou', C. Fusch'

'Pediatrics, McMaster University, Hamilton, Kanada

Background. There are different organisational models to manage a NICU. We recently introduced "Microsystems" and cohorting of patients according to acuity in our level III unit. One outcome parameter to assess the impact of this introduction is the length of rounds. This implementation will create designated areas with more and less intensive care within the NICU. We hypothesize that round length will be shorter after introduction of the new model.

Objectives. To assess the round length during weekdays before and after implementation of Microsystems; to investigate the effect of the implementation of Microsystems on round length in the different areas of the NICU

Methods. Institution: McMaster University NICU, a 47-bed level III; 5 pods with $4 \times 10$ and $1 \times 7$ beds. Successful implementation of Microsystems on May 1st, 2014 after a preparatory phase of 8 months.

Data collection. Prospective collection of daily reports of round lengths (weekdays) for all three teams ( $\left.\mathrm{T}_{1}, \mathrm{~T}_{2}, \mathrm{~T}_{3}\right)$; Time: Data was collected during two periods: 1. pre-implementation phase from Jan- Apr 2014; 2. post-implementation phase from June - Sept 2014. Descriptive analysis using standard statistical methods.

Results. For the two acute teams rounds started earlier and more punctual (T1: 10.06am $\pm 10 \mathrm{~min}$; T2: 10.08am \pm 10 min compared to T1: 10:32am $\pm 20 \mathrm{~min}$; $\mathrm{T} 2: 10: 33 \pm 15 \mathrm{~min}$; $\mathrm{T}_{3}: 10.28 \mathrm{am} \pm 20 \mathrm{~min}$ ); the new intermediate care (IMC) team started later (as planned) and less punctual (not planned) $\mathrm{T}_{3}:$ 13:22 $\pm 30 \mathrm{~min}$. Round length decreased in all three teams (12, 6 and $41 \mathrm{~min}$, respectively), on average by $20 \mathrm{~min}$ per team. $\mathrm{Cu}-$ mulative gain in efficiency 1 hour per day. This translates approximately into 8 hours per day per medical staff.

Conclusion. Round length was impacted by the organisational model applied. The average reduction in cumulative rounding time with the microsystems model corresponds to one $100 \%$ FTE, or in other words would free up the work time of one FTE thereby increasing efficiency of the medical team.

Acknowledgement. The project is funded by HAHSO.

\section{P07-05}

\section{Circadianer und ultradianer Biorhythmus bei Frühgeborenen}

F. Dorn', L. Wirth', S. Gorbey², M. Wege ', M. Zemlin', B. Lemmer ${ }^{5}$, R. Maier ${ }^{6}$ ${ }^{1} K$ linik für Kinder- und Jugendmedizin der Philipps-Universität Marburg, Marburg, Deutschland, 'Institut für Exp. \& Klin. Pharmakologie \& Toxikologie Mannheim der Universität Heidelberg und CBTM, Centrum für Biomedizin und Medizintechnik Mannheim, Medizinische Fakultät Mannheim der Universität Heidelberg, Mannheim, Deutschland, ${ }^{3}$ Phillips Universität Zentrum für Kinderheilkunde, Marburg, Deutschland, ${ }^{4}$ Universitätsklinikum Gießen und Marburg, Standort Marburg, Zentrum für Kinder- und Jugendmedizin, Marburg, Deutschland, ${ }^{5}$ Institut für Exp. \& Klin. Pharmakologie \& Toxikologie Mannheim der Universität Heidelberg, Mannheim, Deutschland, ${ }^{6}$ Universitätsklinikum Marburg, Zentrum für Kinder und Jugendmedizin, Marburg, Deutschland

Hintergrund. Viele physiologische Funktionen wie Hormonsekretion, Schlaf-Wach-Rhythmus und Körpertemperatur unterliegen einem sich regelmäßig wiederholenden Muster, dem biologischen Rhythmus. Umwelteinflüsse wie Licht, Temperatur, Nahrungsaufnahme und soziale Kontakte stellen externe Zeitgeber dar, die zur Ausbildung biologischer Rhythmen beitragen und endogene Rhythmen fortwährend synchronisieren. Bereits beim Feten können circadiane Rhythmen, noch kontrolliert durch die Mutter, beobachtet werden. Nach der Geburt muss sich die ,innere Uhr“ erst entwickeln.
Fragestellung. Wie verhält sich der biologische Rhythmus von Aktivitäts-Ruhe-Mustern und Cortisolkonzentration bei Frühgeborenen in den ersten 4 Lebenswochen?

Methodik. Bei 62 stabilen Frühgeborenen (Gestationsalter $30<37$ SSW, postnatales Alter $<10$ Tage bei Studienbeginn) wurde nach Einwilligung der Eltern an den Studientagen 1, 7 und 14 jeweils über einen Zeitraum von 24 Stunden das Aktivitäts-Ruhe-Verhalten mit Hilfe eines Aktometers aufgezeichnet. Bei 20 dieser Frühgeborenen wurden zusätzlich an den 3 genannten Studientagen zu je sieben standardisierten Zeitpunkten Speichelproben für die Bestimmung (ELISA) der Cortisolkonzentration entnommen. Die Lichtverhältnisse wurden dokumentiert. Die Berechnung der Tag-Nacht-Rhythmik erfolgte mittels der Lomb-Scargle-Methode [Least Squares Spectral Analysis (LSSA)].

Ergebnisse. Eine circadiane Rhythmik für Cortisol konnte nur bei einem Frühgeborenen am ersten Studientag beobachtet werden. Am ersten Studientag war die Cortisolkonzentration im Mittel des gesamten Tages $(=24 \mathrm{~h})$ höher als an den darauffolgenden Studientagen (Gesamttag 1: Median=16,5 nmol/L, Gesamttag 7: Median=9,8 nmol/L, p<0,oo1; Gesamttag 14: Median=10,0 nmol/L, p<0,005), wobei am ersten Studientag besonders die nächtliche Cortisolkonzentration erhöht war (nachts Tag 1: Median=17,1 nmol/L; tags Tag 1: Median=12,9 nmol/L). Beim Aktivitäts-Ruhe-Verhalten dominierte ein ultradianer Rhythmus mit einer vierstündigen Periodenlänge in den ersten Lebenswochen (bei 46 von 151 erhobenen Aktivitätsaufzeichnungen). Bei 12 Kindern konnte an einzelnen Studientagen eine Periodenlänge von 24 Stunden beobachtet werden. Im Verlauf nahm der prozentuale Anteil von Ruhephasen zu (Studientag 1: 55\%; Studientag 7: 61\%, p<0,01; Studientag 14: 60\%, p<0,05). Die durchschnittliche Gesamtschlafzeit verlängerte sich von $66 \%$ an Studientag 1 auf $72 \%$ an Studientag $7(\mathrm{p}<0,05)$ bzw. 69\% an Studientag 14 .

Schlussfolgerung. In den ersten 4 Lebenswochen konnte bei Frühgeborenen noch keine stabile circadiane Rhythmik gefunden werden. Hinsichtlich des Aktivitäts-Ruhe-Musters zeigte sich eine dominierende ultradiane Rhythmik von vier bis sechs Stunden.

\section{P07-06}

\section{Videofeedback in der Neonatologie - ein stationäres Angebot} zur Stärkung der elterlichen Selbstwirksamkeit?

P. Hinner', C. Hócza', M. Sommerfeld', J. Reichert', M. Rüdiger'

'Universitätsklinikum Carl Gustav Carus, Klinik und Poliklinik für Kinderund Jugendmedizin, Fachbereich Neonatologie/Pädiatrische Intensivmedizin, Dresden, Deutschland

Hintergrund. Auf neonatologischen Stationen besteht zunehmend der Anspruch, Eltern frühzeitig Kontakt und Sicherheit im Umgang mit ihrem Kind zu vermitteln und so die Eltern-Kind-Interaktion zu befördern. Damit letzteres erreicht werden kann, ist neben der Vermittlung von Wissen und Handlungskompetenzen auch die Stärkung der elterlichen Selbstwirksamkeit von wesentlicher Bedeutung (Teti, HusseyGardner, \& Hess, 2004). Am Universitätsklinikum Dresden wird den Eltern neben der praktischen Anleitung zur Versorgung des Kindes ein Videofeedback angeboten. Dabei sollen gelungene Interaktionsmomente zwischen Eltern und Kind positiv hervorgehoben und das selbstentdeckende Lernen der Eltern bzgl. kindlicher Verhaltenssignale bestärkt werden.

Fragestellung. Wie bewerten Eltern das Angebot einer Videoaufnahme als auch -auswertung während des stationären Aufenthalts des Kindes? Material und Methoden. Ein 30-minütiges Videofeedback erfolgt während des stationären Aufenthalts anhand einer Videoaufnahme, die während der Versorgungssituation Baden zusammen mit einem Elternteil erstellt wird. Seit 2011 wird den Eltern ein Evaluationsbogen ausgehändigt. Dieser beinhaltet quantitative Items zur Bewertung des Videofeedbacks; offene Fragen ermöglichen zudem eine qualitative Inhaltsanalyse. Es wurden die Daten einer Pilotstudie aus dem Jahr 2012 ausgewertet.

Ergebnisse. Das Videofeedback wurde bisher 253-mal (Stand Januar 2015) durchgeführt. Die Frühgeborenen (Gestationsalter: Med=28+4; $\left.\mathrm{Q} 1=26+6 ; \mathrm{Q}_{3}=30+3\right)$ waren zum Zeitpunkt der Videoaufnahme im 
Median 42 Tage $\left(\mathrm{Q}_{1}=29\right.$; $\left.\mathrm{Q} 3=59\right)$, bzw. korrigiert 34+6 SSW $\left(\mathrm{Q}_{1}=33+5\right.$; $\left.\mathrm{Q}_{3}=36+5\right)$ alt. Von 70 Videofeedbacks des Jahres 2012 liegen für 48 Sitzungen Evaluationsbögen von 64 teilnehmenden Müttern und Vätern vor. Die quantitativen Items zeigen, dass $87 \%$ ihre Erwartungen an das Videofeedback vollständig erfüllt sahen, die erhaltene Rückmeldung gut anwenden können (90\%), ihr Wohlbefinden gesteigert (81\%) und die eigene Kompetenzwahrnehmung bestärkt wurde (73\%). Die Analyse der offenen Fragen lassen nach Kategorisierung zwei wesentliche Gründe für die positive Bewertung des Videofeedbacks erkennen: Die Interaktion mit dem eigenen Kind gespiegelt zu bekommen und die Auswertung als geschützten Raum zur Selbstreflektion zu erleben. Ausnahmslos würden die Evaluationsteilnehmer das Angebot auch anderen Eltern empfehlen. Diskussion. Das Angebot von Videoaufnahme und -auswertung der Eltern-Kind-Interaktion in einer Versorgungssituation findet hohe Akzeptanz bei den Eltern. Die quantitativen Items bilden durchweg eine positive Bewertung durch die Teilnehmer ab. Die qualitative Inhaltsanalyse weist darauf hin, dass das Videofeedback den Eltern eine differenzierte und positive Selbstwahrnehmung der Interaktion mit dem eigenen Kind ermöglicht.

Schlussfolgerung. Das Videofeedback erscheint als effektives Instrument um Eltern gelungene Interaktionen mit dem eigenen Kind verstärkt erfahrbar zu machen. Inwiefern dies die elterliche Selbstwirksamkeit langfristig steigern und die Qualität der Eltern-Kind-Interaktion befördern kann, ist in Längsschnitt- und Multicenter-Studien zu prüfen.

1. Teti DM, Hussey-Gardner B, Hess CR (2004) Self-efficacy and parenting of highrisk infants: The moderating role of parent knowledge of infant development. Journal of Applied Developmental Psychology 25(4):423-437

\section{P07-07}

Entlassung und Weiterbetreuung von Frühgeborenen und kranken Neugeborenen - transsektorale Bedarfsanalyse

S. Ifflaender' ${ }^{1}$ E. Anders ${ }^{2}$, J. Reichert ${ }^{1}$, M. Rüdiger' ${ }^{1}$, P. Hinner ${ }^{1}$

'Medizinische Fakultät Carl Gustav Carus an der TU Dresden, Fachbereich Neonatologie und Pädiatrische Intensivmedizin, Dresden, Deutschland, ${ }^{2}$ Praxis für Kinder- und Jugendmedizin, Freital, Deutschland

Hintergrund. Die derzeitige Situation in der Nach- und Langzeitbetreuung von Frühgeborenen und Risikoneugeborenen in Deutschland ist von der Vielfältigkeit der Angebote und Anbieter geprägt. Regionalspezifisch und ressourcenabhängig werden sowohl an das Perinatalzentrum assoziierte Angebote (Neonatologen, Neuropädiater) als auch klinikunabhängige, externe Strukturen wie niedergelassene Pädiater und Sozialpädiatrische Zentren genutzt. Eine bedarfsgerechte, evidenzbasierte und einheitliche Sekundärprävention setzt die Kenntnis der Bedarfe und Bedürfnisse beteiligter Professionen voraus.

Fragestellung. I.) Wie sollte aus Sicht niedergelassener Kinderärzte ein strukturierter Übergang in die ambulante Weiterbetreuung Frühgeborener aussehen? II.) Welchen Bedarf sehen Neonatologen bezüglich der ambulanten Weiterbetreuung dieser Kinder?

Methoden. I.) Im Jahr 2013 wurden 150 niedergelassene Pädiater im Freistaat Sachsen nach ihren Anforderungen an ein strukturiertes Entlassungsmanagement Frühgeborener befragt. Ermittelt wurden u.a. der optimale Zeitpunkt der Kontaktaufnahme mit den niedergelassenen Kollegen vor Entlassung sowie bevorzugte Medien zur Kontaktaufnahme. II.) Zusätzlich wurden im Dezember 2014 im Rahmen eines Diskussionsforums 50 in der Neonatologie tätige Ärzte und Ärztinnen aus ganz Deutschland eingeladen, Bedarfe und Bedürfnisse an die ambulante Weiterbetreuung Frühgeborener aus der Perspektive des stationären Sektors zu diskutieren.

Ergebnisse. I.) Aus 100 Antworten niedergelassener Kinderärzte ergaben sich folgende Bedarfe bezüglich der Entlassungsplanung: 68\% wünschen sich eine Kontaktaufnahme 3-4 Tage vor der Entlassung. Diese sollte durch die Eltern (66\%) und/oder die Klinik (18\%) sowie per Telefon (47\%) und/oder Fax (46\%) erfolgen. Internet und Email spielten als
Medien eine untergeordnete Rolle (5\%). Desweiteren besteht ein Bedarf an telefonischen Rücksprachemöglichkeiten zu festen Zeiten, sowie der zeitnahen Beantwortung von Rückfragen. II.) Im Rahmen des Diskussionsforums wurden folgende Ziele einer Langzeitbetreuung ehemaliger Frühgeborener identifiziert: 1) Effektive Sekundärprävention von Folgeproblemen der Frühgeburtlichkeit, 2) Kenntnis von Langzeitverläufen zum Zweck der Qualitätssicherung und Ausbildung in der Neonatologie sowie 3) Information, Unterstützung und Förderung der Selbstkompetenz von Eltern Frühgeborener.

Diskussion. Bei der transsektoralen Zusammenarbeit kommt es ambulant tätigen Kinderärzten vor allem auf kurze Kommunikationswege an. Stationär tätige Kollegen befürworten eine strukturierte, evidenzbasierte und risikoadaptierte Vorsorge, die neben der individuellen $\mathrm{Pa}-$ tientenversorgung auch der Qualitätssicherung und der Unterstützung der Familien Frühgeborener dient.

Schlussfolgerung. Eine strukturierte Weiterbetreuung beginnend beim transsektoralen Übergang muss in Absprache mit Akteuren in der Region etabliert werden. Weitere Forschung ist notwendig um eine risikoadaptierte, strukturierte Vorsorge zu entwickeln.

\section{P07-08}

\section{Endlich zu Hause?! Familiäre Anpassung als Frage mütterlicher} Selbstwahrnehmung und kindlicher Verhaltensanforderungen

P. Hinner', J. Hennig', J. Petzoldt' ${ }^{2}$, M. Rüdiger', J. Reichert ${ }^{1}$

'Universitätsklinikum Carl Gustav Carus, Fachbereich für Neonatologie/ Pädiatrische Intensivmedizin, Dresden, Deutschland, ${ }^{2}$ Institut für Klinische Psychologie und Psychotherapie, Technische Universität Dresden, Dresden, Deutschland

Hintergrund. Die Entlassung aus der Klinik ist nicht nur ein wesentlicher Schritt in der medizinischen Behandlung frühgeborener Kinder; für die Familie stellt der Übergang in das Leben zu Hause Wunsch und Herausforderung zugleich dar. Die hohe psychische Belastung während und zum Ende des stationären Aufenthalts ist bereits gut belegt; es bleibt die Frage, welche Aspekte in der ersten Zeit des Übergangs eine Rolle in der psychosozialen Anpassung der Mütter spielen. Dabei werden im Folgenden auch Verhaltensaspekte des Kindes näher betrachtet. Fragestellung. Welche Arten von Belastungen berichten Mütter vier Wochen nach Entlassung und welche Rolle spielt dabei das kindliche Regulationsverhalten (Schreien, Schlafen und Füttern)?

Material und Methoden. Mütter, deren Kinder am Universitätsklinikum Dresden vor der 37. SSW geboren wurden, erhielten 4 Wochen nach Entlassung einen Fragebogen als Online- oder Paper-pencil-Version. Erfasst wurde unter anderem das Eltern-Belastungsinventar (Tröster, 2011) und das Schreien, Schlafen und Füttern des Kindes, angelehnt an den Baby-DIPS (Schneider \& Wolke, 2007).

Ergebnisse. 49 von 61 Müttern beantworteten den Fragebogen, davon 11 Zwillingsmütter. Die Kinder wiesen im Median ein GA von 32 SSW $\left(\mathrm{Q} 1=29 ; \mathrm{Q}_{3}=34\right)$. Es zeigt sich eine hohe mütterliche Belastung aufgrund einer wahrgenommenen Distanz gegenüber dem Kind (35\%) und Selbstzweifeln, der Mutterrolle gerecht zu werden (54\%). In Bezug auf das Kind werden erhöhte Anforderungen in Betreuung und Versorgung (47\%) und Anpassungsschwierigkeiten des Kindes an den häuslichen Alltag (26\%) als belastend beschrieben. Basierend auf mütterlichen Angeben, bestehen bei $20 \%$ der Kinder Probleme beim Einschlafen und bei 31\% Probleme im Essverhalten; 5\% zeigen Hinweise auf exzessives Schreiverhalten. Aus der subjektiven Perspektive fühlen sich jeweils knapp $17 \%$ der Mütter aufgrund des Schlaf- bzw. Schreiverhaltens und $9 \%$ aufgrund des Fütterns gestresst.

Diskussion. Jede zweite Mutter äußert eine hohe Belastung aufgrund der Anforderungen des Kindes und Selbstzweifeln diesen gerecht zu werden. Demgegenüber steht das überwiegend geringe Belastungserleben im Zusammenhang mit den kindlichen Verhaltensbereichen Schreien, Schlafen und Essen. Die Selbstwahrnehmung der Mütter, insbesondere der eigenen elterlichen Kompetenz und emotionalen Nähe zum Kind, 
scheint demnach eine größere Bedeutung für das Belastungserleben zu haben, als das kindliche Regulationsverhalten.

Schlussfolgerung. Für das langfristige Gelingen der Co-Regulation zwischen Eltern und Kind spielt das elterliche Selbstvertrauen eine wesentliche Rolle (Papoušek u. Papoušek, 1987). So ergibt sich für die Nachsorge der Frühgeborenen und ihrer Familien der Anspruch, neben Entwicklungsthemen des Kindes, auch die psychische Anpassung der Eltern und insbesondere deren elterliche Kompetenzwahrnehmung in den Blick zu nehmen.

1. Papoušek H, Papoušek M (1987) Intuitive parenting: a dialectic counterpart to the infant's integrative competence. In J.D. Osofsky (Ed.), Handbook of infant development (2nd ed., pp. 669-713). New York: Wiley.

2. Schneider S, Wolke D. (2007) Diagnostic Interview to assess regulatory disorders in infancy and toddlerhood (Baby-DIPS). Basel: University of Basel.

3. Tröster H. (2011) Eltern-Belastungs-Inventar. Deutsche Version des Parenting Stress Index (PSI). Göttingen: Hogrefe.

\section{P07-09}

\section{Fünf Jahre psychologisch-sozialmedizinische Versorgung an einem Perinatalzentrum - I. Auswirkungen auf die postnatale Entwicklung von frühgeborenen Kindern}

\section{Dehmel', P. Hinner', S. Ifflaender', J. Dinger', J. Schmitt ${ }^{2}$, M. Rüdiger', J.} Reichert ${ }^{\prime}$

'Medizinische Fakultät Carl Gustav Carus an der TU Dresden, Fachbereich Neonatologie und Pädiatrische Intensivmedizin, Dresden, Deutschland, ${ }^{2}$ Medizinische Fakultät Carl Gustav Carus an der TU Dresden, Zentrum für Evidenbasierte Gesundheitsversorgung, Dresden, Deutschland

Hintergrund. Der medizinische Fortschritt ermöglicht heute immer mehr das Überleben auch sehr kleiner Frühgeborener. Neben den Anforderungen an deren medizinische Versorgung wachsen auch die Herausforderungen einer adäquaten Begleitung der Eltern. Die Stärkung elterlicher Kompetenzen hat in der Neonatologie daher an Bedeutung gewonnen. Die psychologisch-sozialmedizinische Versorgung von $\mathrm{Fa}$ milien Frühgeborener, bereits während des initialen stationären Aufenthalts, kann hierzu einen Beitrag leisten (Reichert et al. Monatsschr Kinderheilkd 2014, 162:1010-1017).

Zielstellung. Analyse der Auswirkungen einer strukturierten Psychologisch-sozialmedizinischen Versorgung von Familien Frühgeborener (PSV) fünf Jahre nach Einführung an einem Perinatalzentrum Level 1 auf die postnatale Liegezeit (LZ) sowie Entlassungsgewicht- und alter (EG, EA).

Material und Methode. In die Analyse einbezogen wurden alle Kinder der Geburtsjahrgänge 2007/2008 (prä-PSV) und 2012/2013 (post-PSV) mit einem Geburtsgewicht $\mathrm{GG}<1500 \mathrm{~g}$ und/oder einem Gestationsalter $\mathrm{GA}<33$ SSW (VLBW); sehr untergewichtige Kinder (ELBW, $\mathrm{GG}<1000 \mathrm{~g}$ ) wurden gesondert betrachtet. Ausgeschlossen waren Kinder, die während des Aufenthaltes verstarben oder in eine andere Klinik verlegt wurden; kontrolliert wurde der Gesundheitszustand (BPD, NEC, ROP, IVH).

Ergebnisse. In den Gesundheits- und Geburtsparametern unterschieden sich die Analysegruppen nicht signifikant. Von insgesamt 188 VLBW-Kindern der prä-PSV wurden 140 (74,5\%) einbezogen, die im Mittel nach $75 \mathrm{~d}$ mit einem $E G=2.410 \mathrm{~g}$ und einem $E A=39+2$ SSW entlassen wurden (69 ELBW dieser Gruppe: $\mathrm{LZ}=98 \mathrm{~d}, \mathrm{EG}=2.511 \mathrm{~g}, \mathrm{EA}=41+3$ SSW). In der Gruppe post-PSV wurden 138 von 205 VLBW-Kindern $(67,3 \%)$ berücksichtigt. Die Entlassung nach Hause erfolgte nach $65 \mathrm{~d}$ mit $\mathrm{EG}=2.421 \mathrm{~g}$ und $\mathrm{EA}=37+5 \mathrm{SSW}$ (66 ELBW dieser Gruppe: $\mathrm{LZ}=82 \mathrm{~d}$, $\mathrm{EG}=2.505 \mathrm{~g}, \mathrm{EA}=38+5 \mathrm{SSW}$ ).

Diskussion. Nach PSV-Einführung wurden VLBW-Kinder etwa 10 Tage früher $(t=2,313 ; p=0.022)$ nach Hause entlassen, was sich auch im Entlassungsalter von $37+5$ vs. $39+2$ SSW $(t=3,647 ; p=0.000)$ widerspiegelt; Unterschiede in den Entlassungsgewichten fanden sich nicht. Ähnliche Ergebnisse zeigten sich bei den ELBW-Kindern $(\Delta=16 \mathrm{~d}, \mathrm{t}=2,757, \mathrm{p}=.007$; $38+5$ vs. $41+3$ SSW, $t=3,962, p=0.000$; keine Gewichtsdifferenzen). Die Daten verweisen auf eine relative Entwicklungsbeschleunigung der
Kinder, die bei verringerter Verweildauer ähnliche Entlassungsgewichte erreichten. Hierzu könnte die Kompetenzsteigerung der Eltern durch eine adäquate PSV beigetragen haben.

Schlussfolgerung. Die Ergebnisse stützen die Annahme, eine PSV von Familien Frühgeborener wirkt sich positiv auf die unmittelbare pränatale Entwicklung der Kinder aus. Eltern pflegen und versorgen ihr Kind frühzeitig weitgehend selbstständig und sehen sich eher in der Lage, es zu Hause zu versorgen. Welchen Beitrag eine strukturierte PSV vor dem Hintergrund der medizinischen Behandlung Frühgeborener leistet, bleibt noch zu prüfen.

\section{P07-10}

Notwendige Ressourcen zur Durchführung stationärer und ambulanter psychosozialer Beratung in der Neonatologie

B. Hüning', M. Reimann², S. Sahlmen², S. Leibold'2, J. Nabring', U. FelderhoffMüsert

'Universitätsklinikum Essen, Klinik für Kinderheilkunde I, Neonatologie, Zentrum für Kinder- und Jugendmedizin, Essen, Deutschland, ${ }^{2}$ Universitätsklinikum Essen, Elternberatung „Frühstart"/Bunter Kreis am Universitätsklinikum Essen, Essen, Deutschland, ${ }^{3}$ Universitätsklinikum Essen, Medizinisches Controlling, Essen, Deutschland, ${ }^{4}$ Universitätsklinikum Essen, Direktorin der Klinik für Kinderheilkunde I, Zentrum für Kinder- und Jugendmedizin, Essen, Deutschland

Hintergrund. Die neonatologische Versorgung Frühgeborener hat sich in den letzten Jahrzehnten stark gewandelt. Mit dem GBA-Beschluss von 2013 erkennt die Politik die Notwendigkeit psychosozialer, familienzentrierter Betreuung in der Neonatologie an. Ziel dieser Untersuchung ist es, die hierfür notwendigen personellen und zeitlichen Ressourcen an einem Zentrum mit etablierter familienzentrierter stationärer und ambulanter Betreuung zu evaluieren.

Methodik. Die Elternberatung „Frühstart/Bunter Kreis“ betreut Frühgeborene und kranke Neugeborene von der Risikoschwangerschaft bis in die Häuslichkeit mit demselben interdisziplinären Team. Über einen Zeitraum von 3 Jahren wurden die einzelnen Phasen der Betreuung aller neonatologischen Fälle hinsichtlich der stattgefundenen Kontakte/ Dauer untersucht. Für die Fälle aus 2014, die noch betreut werden, wurden die Kontakte/Dauer bis zum 31.1.15 ausgewertet. Gleichzeitig wurde die personelle Ausstattung pro Quartal und Fälle, die Inanspruchnahme der Leistungen durch die Hauptzielgruppe Frühgeborene $<32$. SSW und die Finanzierung (auf der Basis sozialmedizinischer Nachsorge $\$ 43,2$ SGB V oder über private Spenden) eruiert.

Ergebnisse. Vom 1.1.2012 bis 31.12.2014 wurden 372 Fälle (1601 vollstationäre Fälle insgesamt) im Rahmen der Elternberatung betreut. Die Inanspruchnahme durch die Hauptzielgruppe (Frühgeborene $<32$ SSW) lag im Mittel bei 89\% (84-97\%). Der zeitliche Aufwand für die psychosoziale Betreuung präpartal bis ambulant ist in der Gruppe der Kinder mit angeborenen Fehlbildungen mit im Mittel (MW)13,4 Stunden (h) am höchsten; schwer kranke NG: MW 13 h, sehr kleine FG: 12,9 h. Die Phase des Übergangs in die Häuslichkeit und die ambulante Betreuung nehmen die größte Zeit in Anspruch: MW 4,16 h, wohingegen präpartal 1-2 Kontakte ausreichend waren (MW:1,8 h). Mit einem Stellenanteil von 3 Vollzeitkräften (VK), aufgeteilt auf mehrere Teilzeitkräfte, können bis zu 54 Familien im Quartal betreut werden (MW 48). Die Finanzierung der ambulanten Besuche auf Basis privater Spenden konnte von $24,1 \% 2012$ auf 5,3\% 2014 gesenkt werden, gleichzeitig wurden $95 \%$ (MW 2012/2013) der Anträge zur sozialmedizinischen Nachsorge bei den Krankenkassen erfolgreich gestellt.

Schlussfolgerung. Die psychosoziale Betreuung sehr kleiner Frühgeborener wird gut durch die Eltern angenommen und liegt zeitlich gesehen in einem überschaubaren Rahmen. Mit 115,5 Wochenstunden (3VK) Elternberatung können im Durchschnitt pro Quartal 48 Familien betreut werden. 


\section{Posterwalk 8 - Infektiologie}

\section{P08-01 \\ Granulomatosis infantiseptica: Early-onset-Listeriose eines Früh- geborenen mit kutaner Hautbeteiligung - ein Fallbericht \\ B. Bubl', M. Nelle \\ 'Medizinische Kinderklinik/Inselspital, Bern, Schweiz}

Hintergrund. Listerieninfektionen kommen hauptsächlich bei immunsupprimierten Personen und Schwangeren vor. Die Early-onset-Listeriose beim Neugeborene eine seltene aber lebensbedrohliche Erkrankung.

Fallbericht. Aufgrund eines tachykarden CTGs mit mütterlichem CRPAnstieg und vorzeitigen Wehen wurde ein weibliches Frühgeborenes in der $33+0$ SSW per eiliger Sectio zur Welt gebracht. Postnatal zeigte sich ein septisches Frühgeborenes mit muskulärem Hypotonus, Atemnotsyndrom und disseminierten Hauteffloreszenzen. Die Plazenta war grünlich und hatte belegte Eihäute. Die Laborwerte des Neugeborenen waren mit einer Linksverschiebung von $60 \%$, einem erhöhtem CRP ( $\max$. CRP $112 \mathrm{mg} / \mathrm{l}$ ) und einer Thrombozytopenie von 50 G/l nach Geburt auffällig. Aus diesen Gründen erfolgte postnatal der sofortige Beginn einer Antibiotikatherapie mit Amoxicillin und Amikacin. Der Verdacht der Sepsis wurde in der mikrobiologischen Diagnostik bestätigt. Sowohl die Blutkultur des Kindes, als auch die Plazenta wiesen Listerien (Listeria monozytogenes) auf. Die Ergebnisse der Lumbalpunktion des Frühgeborenen waren unauffällig. Die Haut wies ubiquitär Effloreszenzen mit kleinen blassen Pusteln auf, welche erythematös umrundet waren. Dies passt in das klinische Bild der seltenen kutanen Hautbeteiligung im Rahmen einer Early-Onset Listeriensepsis (Granulomatosis infantiseptica). Unter der Antibiotikatherapie über zehn Tage besserte sich der Allgemeinzustand des Kindes rasch. Weiter verschwanden sowohl die Hauteffloreszenzen, und die erhöhten Entzündungswerte normalisierten sich. Anamnestisch ließ sich bei der Mutter eruieren, dass sie in der Schwangerschaft unpasteurisierten Weichkäse $\mathrm{zu}$ sich genommen hatte.

Schlussfolgerung. Unpasteurisierte Milchprodukte sind von Schwangeren und immunsupprimierten Personen zu vermeiden. Beim septischen Neugeborenen mit pustulösem Hautausschlag sollte auch eine Listerieninfektion in Betracht gezogen werden.

\section{P08-02}

\section{Rekurrierendes Staphylococcal-Scalded-Skin-Syndrom bei einem Frühgeborenen}

\section{B. Kühne', A. Schmidt ${ }^{2}$, A. Kribs ${ }^{3}$}

${ }^{1}$ Klinik und Poliklinik für Kinder- und Jugendmedizin, Universitätsklinikum Köln, Köln, Deutschland, ${ }^{2}$ Uniklinik Köln, Klinik und Poliklinik für Kinderund Jugendmedizin, Neonatologie, Köln, Deutschland, ${ }^{3}$ Universitätskinderklinik Köln, Neonatologie und Pädiatrische Intensivmedizin, Köln, Deutschland

Einleitung. Frühgeborene zeigen neben oftmals im Vordergrund stehenden kardiorespiratorischen und gastrointestinalen Problemen aufgrund einer noch unzureichend ausgebildeten epidermalen Barriere auch eine Anfälligkeit zur Entwicklung lebensbedrohlicher Infektionen ausgehend von der Haut.

Fallbericht. Wir berichten über den Fall eines männlichen Frühgeborenen der 24+1 Schwangerschaftswoche (SSW), Geburtsgewicht $680 \mathrm{~g}$ Postnatal zeigte sich unter nicht-invasiver Atemhilfe zunächst ein unauffälliger klinischer Verlauf. Am 33. Lebenstag Entwicklung eines perioralen Erythems. Innerhalb weniger Stunden Blasenbildung vor allem an druckexponierten Stellen mit Irritabilität und septischen Krankheitsbild mit respiratorischem Erschöpfen. Notwendigkeit einer sechstägigen invasiven Beatmung sowie neuntägiger antibiotischer in travenöser Therapie; initial empirisch mit Ceftazidim und Vancomycin. Nach Nachweis von Methicillin-sensiblen Staphylococcus aureus
(S. aureus) in diversen Haut- und Schleimhautabstrichen, sowie in einem Hautblasenpunktat, erfolgte eine Umstellung von Vancomycin auf Flucoxacillin. Unter diesem Therapieregime komplette Rückbildung des Staphylococcal Scalded Skin Syndroms (SSSS). Am 87. Lebenstag erneut beginnende Hautablösungen an Finger- und Fußkuppen, Gesicht und in den Leistenregionen. Aufgrund der Anamnese und des klinischen Bildes Verdacht auf ein Rezidiv des SSSS und Start einer intravenösen antibiotischen Therapie mit Cefuroxim und Vancomycin. Bei erschöpften Venenverhältnissen Umstellung nach 3 Tagen auf Amoxicillin/Clavulansäure, mit Fortführung für weitere 20 Tage. Die Rachenabstriche der Eltern waren beide positiv für Exfoliatin bildenden S. aureus. Nachweis des gleichen S. aureus Stamms im mütterlichen und kindlichen Abstrich, sodass sich der Verdacht einer Ping-Pong-Infektion bestätigte.

Schlussfolgerung. Das SSSS, was in der Literatur auch nach den Namen der Erst- und Zweitbeschreiber Dermatitis exfoliativa neonatorum Ritter von Rittershain oder staphylogenes Lyell-Syndrom genannt wird, stellt die sekundäre Hautmanifestation einer initial lokalen Infektion beziehungsweise Besiedelung durch den S. aureus dar. Meist erkranken Neugeborene und Säuglinge. Vermutlich aufgrund ihrer noch physiologischen Unreife des Immunsystems (unter anderem Fehlen von protektiven Antitoxinen) sowie der noch verminderten renalen Toxinclearence. Darüber hinaus ist auch die Anzahl der Besiedelungen mit S. aureus wesentlich höher als im Erwachsenenalter. Bei dem von uns beschriebenen Fall gab es zu keiner Zeit Hinweise auf eine zugrundeliegende Erkrankung, welche das Immunsystem des Frühgeborenen oder seine Nierenfunktion negativ, und somit die Entstehung eines SSSS sowie dessen Verlauf beeinflussen würde. In unserem Fall erfolgte die ReExposition durch eine Ping-Pong-Infektion zwischen Mutter und Kind. Dies hebt die Bedeutung von mikrobiologischen Umgebungsuntersuchungen bei rekurrierenden Hautinfektionen hervor.

\section{P08-03}

Letal verlaufende Co-Infektion durch Influenza $A$ und PVL-MSSA

T. Ott' ${ }^{1}$, W. Beckmann', K. Wiebe' ${ }^{2}$ J. Wüllenweber', K. Masjosthusmann', E. Rieger-Fackeldey', G. Rellensmann ${ }^{5}$, H. Omran ${ }^{7}$

'Westf. Wilhelms-Univ.- Kinderklinik, Klinik für Kinder- und Jugendmedizin - Allgemeine Pädiatrie -, Münster, Deutschland, ${ }^{2}$ Westf. WilhelmsUniv.- Kinderklinik, Department für Herz- und Thoraxchirurgie - Sektion Thoraxchirurgie, Münster, Deutschland, ${ }^{3}$ Westf. Wilhelms-Univ.- Klinik, Institut für klinische Mikrobiologie, Münster, Deutschland, ${ }^{4}$ Universitätsklinikum Münster, Klinik und Poliklinik für Kinder- und Jugendmedizin, -Allgemeine Pädiatrie-, Neonatologie und Pädiatrische Intensivmedizin, Münster, Deutschland, ${ }^{5}$ Universitätsklinikum Münster, Klinik und Poliklinik für Kinderheilkunde und Jugendmedizin, Allgemeine Pädiatrie, Münster, Deutschland

Wir zeigen den Fall eines 13-jährigen Jungen ohne Vorerkrankungen mit einer Pneumonie und schwersten septischen Schock bei einer CoInfektion durch Influenza Typ A und einen PVL-bildenden S. aureus. Nach kurzem Krankheitsverlauf über 2 Tage wurde der Patient mit einem perakut verlaufenden Kreislaufversagen aufgenommen, neben einer respiratorischen Insuffizienz bestand eine ausgeprägte DIC. Die Entzündungszeichen waren massiv erhöht (PCT 2297 ng/ml). Bei schwerem ARDS wurde nach 2 Tagen eine vv-ECMO begonnen, nach 4 Tagen eine Nierenersatztherapie. Nach 9 Tagen verstarb der Patient an einem generalisierten Hirnödem mit Einklemmung. Als Erreger ließ sich Influenza Typ A $\mathrm{H}_{3}$ identifizieren, zusätzlich ein S.-aureus-Stamm, bei dem die Bildung von Panton-Valentine-Leucozidin nachgewiesen werden konnte. Eine Infektion durch Influenza-Viren mit bakterieller Superinfektion zeichnet sich häufig durch ein schwer verlaufendes Krankheitsbild aus. Insbesondere die Ko-Infektion von Influenza mit PVL-bildenden S.-aureus-Stämmen wurde wiederholt mit foudroyanten Verläufen mit hoher Mortalität assoziiert. Ein mögliches Erklärungsmodell beinhaltet $u$. a. die durch die virale Zellschädigung ausgelöste Infiltration durch Neutrophile, die durch die bakteriellen Toxine 
geschädigt werden, was im Sinne eines Toxic-shock-Syndroms zu einer massiven Freisetzung von Proteasen und Aktivierung inflammatorischer Kaskaden führt.

\section{P08-04 \\ Immunglobuline zur Therapie von Toxin-vermittelten Erkran- kungen bei durch Staphylococcus-aureus-besiedelten Frühge- borenen \\ V. Boos', A. Reinhold' , B. Piening ${ }^{3}$, P. Koehne ${ }^{4}$, C. Dame', C. Bührer', T. Schmitz ${ }^{2}$ \\ ${ }^{1}$ Charité - Universitätsmedizin Berlin, Klinik für Neonatologie, Berlin, Deutschland, ${ }^{2}$ Charite Kliniken $\mathrm{f}$. Kinderheilkunde und Kinderchirurgie, Klinik für Neonatologie, Berlin, Deutschland, ${ }^{3}$ Charité, Institut für Hygiene und Umweltmedizin, Berlin, Deutschland, ${ }^{4}$ Charité Campus Virchow-Klinik, Medizinische Fakultät der Humboldt-Universität zu Berlin, Berlin, Deutsch- land, ${ }^{5}$ Charité - Universitätsmedizin Berlin, Campus Virchow-Klinikum, Neonatologie, Berlin, Deutschland}

Einleitung. Auf neonatologischen Stationen sind Staphylokokken häufige Erreger bei Katheter-assoziierten Infektionen. Besiedlungen oder Infektionen durch Staphylococcus aureus können bei Frühgeborenen durch Bildung von Toxinen zur Ausbildung des Staphylococcus Scaled Skin Syndome (SSSS), Toxic Shock Syndrome (TSS) oder der Neonatal TSS-like Exanthematous Disease (NTED) führen.

Kasuistik. Eutrophes, männliches Frühgeborenes (23 4/7 Schwangerschaftswoche, Geburtsgewicht 588 g) mit Bronchopulmonaler Dysplasie und Frühgeborenenretinopathie. Seit dem 35. Lebenstag wiederholt Nachweis von Staphylococcus aureus im Rachenabstrich. Ab dem 71 Lebenstag rasche klinische Verschlechterung, mit Intubation und maschineller Beatmung über insgesamt 9o Stunden. Bei erhöhtem CRP (40,0 mg/L) und Verdacht auf Sepsis Beginn einer antibiotischen Therapie mit Meropenem und Vancomycin. Bei Entwicklung einer pulmonalen Hypertonie Beatmung mit iNO sowie bei weiterer dramatischer Verschlechterung und bekannter Besiedlung durch Staphylococcus aureus einmalig Gabe von Immunglobulinen (Gamunex ${ }^{\infty}$ 10\%, Fa. Grifols, Frankfurt/Main) sowie Therapieerweiterung um Fluconazol bzw. im Verlauf Amphotericin B. Das CRP stieg im Verlauf auf maximal 212,5 mg/L am 74. Lebenstag an. Am 79. Lebenstag Auftreten von zum Teil großflächigen Hautablösungen an den Finger- und Zehen, welche nach wenigen Tagen abheilten. Orale oder mukosale Läsionen traten nich auf. Nach den positiven Rachenabstrichen gelang bei dem intubierten Kind nun auch im Trachealsekret der Nachweis von Staphylococcus aureus. In der Blutkultur Nachweis von Staphylococcus capitis, bei dem der Nachweis der genetischen Determinanten für die Bildung der exfoliativen Toxine A und B (ET-A und ET-B) und des Panton-Valentine-Leukozidins (lukPV) am Nationalen Referenzzentrum (NRZ) für Staphylokokken negativ war. Die genetischen Determinanten wurden für den Staphylococcus aureus nicht bestimmt.

Diskussion. Auch wenn bei dem isolierten Staphylococcus aureus keine Toxinbildung untersucht wurde, so ist in der Gesamtschau aufgrund des raschen klinischen Verfalls des Patienten und der 8 Tage danach aufgetretenen Blasen und Schuppungen der Haut von einer durch Toxine des Staphylococcus aureus vermittelten Erkrankung auszugehen. Der in der Blutkultur isolierte Staphylococcus capitis ist eher als Kontamination zu werten, der Keim ist nicht als Toxinbildner beschrieben Die Erkrankung wurde bei unserem Patienten erfolgreich mit Antibiotika und Immunglobulinen behandelt. Da Immunglobulin-Präparate auch spezifische Antikörper gegen Staphylokokkentoxine enthalten, ist bei bekannter Besiedlung mit Staphylokokken und klinisch beginnender Sepsis die Gabe von intravenösen Immunglobulinen sinnvoll, um im Fall einer Freisetzung von Staphylokokkentoxinen diese zu binden und die Schwere der Erkrankung abzumildern. Auch eine prophylaktische Gabe bei Hochrisikopatienten ohne ausreichend Nestschutz erscheint sinnvoll. Antibiotikum der Wahl ist Clindamycin, da es im Gegensatz zu ß-Lactam-Antibiotika die Toxinproduktion der Staphylokokken hemmt.

\section{P08-05}

\section{Enterovirusinfektion - Differentialdiagnose des akuten Abdo-} mens bei einem Frühgeborenen

N. Lüsebrink', H. Buxmann', B. Wittekindt', S. Gfrörer', O. Keppler ', M. Wittschorek $^{4}$ R. Schlößer ${ }^{2}$

'Klinikum der J.W. Goethe Universität, Klinik für Kinder- und Jugendmedizin, Neonatologie, Frankfurt am Main, Deutschland, ${ }^{2}$ Klinikum der J.W. Goethe-Univ. Zentrum der Chirurgie, Kinderchirurgie, Frankfurt, Deutschland, ${ }^{3}$ Klinikum der J.W. Goethe-Univ., Institut für Medizinische Virologie, Frankfurt, Deutschland, ${ }^{4}$ Stadtkrankenhaus, Kinderklinik, Hanau, Deutschland

Einleitung. Bei einer raschen Verschlechterung des Allgemeinzustandes mit abdominellem Fokus bei Früh- und reifen Neugeborenen sind zahlreiche Differentialdiagnosen zu bedenken: Komplikationen auf der Basis angeborene Fehlbildungen des Gastrointestinaltraktes, systemische Infektionen, nekrotisierende Enterokolitis (NEK) sowie Stoffwechselerkrankungen, die teilweise spezifische Therapien erfordern.

Fallbericht. Wir berichten über ein Frühgeborenes von $35+1$ SSW, welches am Lebenstag 6 aufgrund eines akuten Abdomens mit dem initialen Verdacht auf NEK zur kinderchirurgischen Mitbetreuung in unser Klinikum verlegt wurde. In der Untersuchung zeigten sich ein druckschmerzhaftes Abdomen, Aszites einhergehend mit laborchemischen Zeichen einer Leberfunktionsstörung (erhöhte GOT 317 U/I) und pathologischen Gerinnungsparametern (TPZ 58\%, PTT 63 s), sowie eine relevante Hämolyse und Thrombopenie (Hb $10 \mathrm{~g} / \mathrm{dl}$, LDH $2020 \mathrm{U} / \mathrm{I}$, Thrombozyten 70/nl). In der Bildgebung fanden sich Nebennierenblutungen beidseits. Radiologisch bestanden keine NEK-typischen Zeichen (keine Pneumatosis, keine freie Luft oder Luft im Pfortadersystem). Aufgrund der Beteiligung diverser Organsysteme wurde eine breite internistische (Ausschluss u. a. von Stoffwechselstörungen, hämolytische Anämien) und infektiologische Abklärung initiiert. Hierbei zeigten sich unauffällige Befunde für die meisten darmpathogenen Keime (Adeno-, Astro-, Rota-, Noro-Virus, Salmonellen, Shigellen, Yersinien, Campylobacter, C. difficile/Toxin, C. perfringens, E. coli, Enterobacter, Pilze). Im Blut und Stuhl des Patienten waren jedoch Enteroviren mittels RT-PCR nachweisbar. Das Enterovirus-IgM im Serum war positiv. Die Virusisolierung ergab ECHO-11-Virus als Enterovirus-Subtyp. Unter konservativ-supportiver Therapie einschließlich der Gabe von Blutprodukten und Immunglobulinen besserte sich der klinische Zustand unseres Patienten. Ab Lebenstag 11 fanden sich stabile Blutbildwerte, ab Lebenstag 15 normale Leber- und Hämolyseparameter. Am Lebenstag 30 wurde der Patient unbeeinträchtigt nach Hause entlassen. Schlussfolgerung. Enterovirusinfektionen beim Neugeborenen können ein Hepatitis-Hämorrhagie-Syndrom verursachen, welches aufgrund einer Mortalitätsrate von $24-31 \%$ und möglichen Blutungskomplikationen eine klinisch hoch relevante Differentialdiagnose des akuten Abdomens bei Früh- und reifen Neugeborenen ist. Die Sicherung der Diagnose kann durch den serologischen und molekularbiologischen Nachweis einer akuten Enterovirus-Infektion im Blut und Stuhl des Patienten erfolgen. Eine spezifische Therapieoption besteht nicht.

\section{P08-06}

\section{Linezolid als Auslöser einer schweren metabolischen Azidose}

M. Marschallek', R. Hennrich', P. Hanff', S. Armbrust ${ }^{2}$

${ }^{1} K$ linikum Neubrandenburg, Klinik für Kinder- und Jugendmedizin, Neubrandenburg, Deutschland, ${ }^{2}$ Dietrich-Bonhoeffer-Klinikum Neubrandenburg, Klinik für Kinder- und Jugendmedizin, Cölpin, Deutschland

Einleitung. Verschiedene Ursachen können beim unreifen Neonaten zur Azidose führen. Antibiotika gehören dabei nicht zur primär verdächtigen Substanzgruppe, wobei einzelne Fälle zumeist außerhalb der Neonatologie beschrieben sind. Wir berichten erstmalig über den Fall einer schweren metabolischen Linezolid-assoziierten Azidose bei einem ELBW-Frühgeborenen. 
Fall. Frühgeborenes $24+2$ SSW, GG: 485 g, NapH 7,3, BE 2, APGAR 5/6/5. Am 16. Lebenstag klinisch krankes Kind mit ansteigenden Entzündungsparametern. Bei vorbehandeltem Kind und Nachweis von methicillinresistentem S. epidermidis Entscheidung zur antibiotischen Therapie mit Piperacillin und Linezolid ( $3 \times 6 \mathrm{mg}$, entsprechend $26 \mathrm{mg} /$ $\mathrm{kg} \mathrm{KG/d)} \mathrm{Mit} \mathrm{Beginn} \mathrm{des} \mathrm{3.} \mathrm{Behandlungstags} \mathrm{Abfall} \mathrm{des} \mathrm{Base} \mathrm{Excess,}$ Abfall des $\mathrm{pH}$ und Anstieg des Lactats. Notwendigkeit der Pufferung mit Beginn des 4. Tages, trotzdem weiter Lactatazidose. Mit Beginn des 5. Tages unter dem V. a. Linezolid-assoziierte metabolische Azidose Beendigung dieser Therapie. Normalisierung der Parameter erst nach über einer Woche.

Diskussion. 2003 wurde erstmalig eine Lactatazidose unter Linezolid bei einer lang andauernden Therapie bei einem Erwachsenen beschrieben. Es gibt anekdotische Fallbeschreibungen letaler Lactatazidosen unter Linezolid bei Hepatopathien in pädiatrischen Patienten. Als Auslöser werden mitochondriale Proteinsynthesestörungen diskutiert. Inwieweit bei unserem Patienten auch die neonatale Unreife von Leber und Niere eine Rolle spielt, bleibt offen. Die vorliegenden pharmakokinetischen Daten beruhen auf nur sehr wenigen Patienten in dieser Altersgruppe.

Schlussfolgerung. Das Lactat und der $\mathrm{pH}$ sollten beim Einsatz von Linezolid engmaschig überwacht werden. Beim Auftreten einer Lactatazidose muss Linezolid als auslösendes Agens in Betracht gezogen werden.

\section{P08-07 \\ Neonatale Encephalitis mit ausgeprägten Marklagerverände- rungen durch Parechoviren - zwei Fallbeispiele}

\section{Jaggy', T. Scheffner', H. Schneider', T. Dütting ${ }^{2}$, P. Freisinger ${ }^{1}$}

'Klinikum am Steinenberg Reutlingen, Klinik für Kinder- und Jugendmedizin, Reutlingen, Deutschland, ${ }^{2}$ Klinikum am Steinenberg, Radiologische Abteilung, Reutlingen, Deutschland

Hintergrund. Enteroviren sind bereits als Ursache einer neonatalen Encephalitis oder viralen Meningitis bekannt. Parechoviren, die ebenfalls zu den Picornaviren gehören, können seit einiger Zeit mittels PCR nachgewiesen werden. Insbesondere $\mathrm{HPeV}_{3}$ wird hierbei als Erreger neonataler Encephalitiden beschrieben. Wir berichten über zwei Neugeborene (NG) mit Encephalitis und ausgeprägten Marklagerveränderungen aufgrund einer Parechovireninfektion.

Fallbeispiel 1. Anamnese: Reifes NG, das am 7. Lebenstag (LT) durch Fieber, schlechtes Trinkverhalten und Irritabilität auffiel. Geburtsverlauf und Schwangerschaftsanamnese waren unauffällig. In der folgenden Nacht Verschlechterung des Allgemeinzustandes mit therapieresistenten Krampfanfällen. Diagnostik: Leicht erhöhtes Il-6 (125 pg/ml), unauffälliger Liquorbefund. Im MRT ausgeprägte Diffusionsstörung im Marklager frontal, okzipital, sowie im Bereich des Balkens. Im Stuh und Liquor Parechoviren (PCR) nachweisbar. Verlauf: Beatmung über 3 Tage, kurzzeitige Trapanalnarkose und antikonvulsive Therapie mit Phenobarbital, klinische Besserung nach 3 Tagen. Im MRT nach 4 Monaten keine Diffusionsstörung, diskrete Narbenbildung. Anfallsfreiheit sowie neurologisch unauffällige Entwicklung nach 1 Jahr.

Fallbeispiel 2. Anamnese: Reifes NG, am 11. Lebenstag Fieber und Irritabilität. Geburtsverlauf und Schwangerschaftsanamnese unauffällig. Der Vater des Jungen hatte einen respiratorischen Infekt, die Schwester Fieber. Am 12. LT kam es zu rezidivierenden therapieresistenten Krampfanfällen. Diagnostik: Leicht erhöhtes Il-6 (59 pg/ml), unauffälliger Liquorbefund. Im MRT deutliche Diffusionsstörungen im Marklager beidseits frontal, temporal sowie im Bereich des Balkens. Im Stuhl und Liquor Parechoviren (PCR) nachweisbar. Verlauf: Antikonvulsive Therapie mit Phenobarbital über 7 Tage sowie Levetiracetam. Langsame klinische Besserung ab dem 13. LT. Mit 1,5 Monaten im MRT keine Diffusionsstörung, frontal im Marklager beidseits porencephale Defekte. Unter Levetiracetam anfallsfrei, neurologisch keine Auffälligkeiten. Vergleich mit publizierten Fallberichten. Verboon-Maciolek et al. (2008): 10 Kindern mit neonataler Encephalitis durch HPeV Typ 3. Im MRT zeigten sich entsprechend der hier beschriebenen Fälle Diffusions- störungen der weißen Substanz. Das Outcome zeigte sich sehr unterschiedlich, bei 50\% der Kinder Restitutio ad integrum. Bei 50\% heterogenes Schädigungsmuster.

Schlussfolgerung. Das klinische Bild einer neonatalen Sepsis mit Irritabilität, schwierig zu therapierenden Krampfanfällen, unauffälligen Entzündungsparametern und fehlender Pleozytose im Liquor sollte an eine Parecho-Encephalitis denken lassen. Im MRT zeigen sich ausgedehnte Diffusionsstörungen im Marklager. Es handelt sich um eine vermutlich unterdiagnostizierte Ursache einer neonatalen Encephalitis und sollte als Differentialdiagnose von NG-Krampfanfällen bedacht werden. Der Nachweis gelingt mit einer PCR Untersuchung auf Parechoviren im Liquor.

\section{P08-08}

Disseminierte neonatale Herpes-simplex(HSV)-1-Infektion mit fehlenden HSV1-IgG-Antikörpern (AK) beim Neugeborenen und hoch positivem HSV1-IgG-AK-Nachweis bei der Mutter

H. Buxmann', K. Schäfer', B. Wittekindt', S. Hünecke' ${ }^{2}$, H. Rabenau' ${ }^{3}$, K. Hamprecht', R. Schlößer ${ }^{1}$

'Klinikum der J.W. Goethe-Univ., Klinik für Kinder- und Jugendmedizin, Neonatologie, Frankfurt am Main, Deutschland, ${ }^{2}$ Klinikum der J.W.G oetheUniv., Klinik für Kinder- und Jugendmedizin, Stammzelltransplantation und Immunologie, Frankfurt am Main, Deutschland, ${ }^{3}$ Institut für Medizinische Virologie, Frankfurt am Main, Deutschland, ${ }^{4}$ Universitätsklinikum Tübingen, Konsiliarlabor für kongenitale Virusinfektionen, Tübingen, Deutschland

Einleitung. Neonatale HSV-Infektionen sind selten (Inzidenz: o,o2o,3\%o). Die klinische Symptomatik beginnt oft unspezifisch und kann meist erst im Verlauf den drei klassischen Formen: lokale Infektion, Infektion des ZNS oder disseminierte systemische Infektion zugeordnet werden. Letztere hat eine hohe Mortalitätsrate von 20-50\%.

Fallbericht. Das männliche Neugeborene bot am Lebenstag drei Fieber, Tachydyspnoe und erhöhtes CrP ( $5 \mathrm{mg} / \mathrm{dl})$. Im Röntgen Thorax zeigten sich Infiltrate. Unter einer Therapie mit Ampicillin, Cefotaxim und Getamicin besserte sich die Symptomatik nicht, sodass ab Lebenstag 5 zusätzlich mit Fluconazol und Aciclovir behandelt wurde. Ampicillin und Cefotaxim wurden durch Vancomycin und Meropenem ersetzt. Das Neugeborene wurde am Lebenstag 8 beatmungspflichtig und entwickelte eine persistierende pulmonale Hypertonie, die eine Hochfrequenzoszillationsbeatmung mit inhalativem Stickstoffmonoxid erforderlich machte. Zu keinem Zeitpunkt bestanden Haut-Effloreszenzen. Im Blut und im Trachealsekret wurde HSV-1 mittels PCR nachgewiesen. Im kindlichen Serum fanden sich keine AK (IgG und IgM) gegen HSV, CMV und Röteln. VZV-IgG war mit $500 \mathrm{IE} / \mathrm{ml}$ nachweisbar bei negativem VZV-IgM. Das Gesamt-IgG des Neugeborenen war mit $758 \mathrm{mg} / \mathrm{dl}$ normwertig. Die Lymphzytensubpopulationen des Neugeborenen wiesen keine spezifische Pathologie auf. Weder klinisch, noch in den Liquoranalysen oder in der cMRT fanden sich Hinweise auf eine HSV-Enzephalitis. In der Familie waren keine Immundefekte bekannt. Die Mutter hatte keine vaginalen Beschwerden. Bei makroskopisch unauffälligem Lokalbefund wurde HSV-1 mittels PCR im Vaginalabstrich der Mutter nachgewiesen. Die mütterlichen AK-Titer im Serum waren: HSV 1/2 IgG > 2500 AE/ml, HSV-2 IgG negativ, HSV 1/2 IgM $120 \mathrm{AE} / \mathrm{ml}$, VZV-IgG 3300 IE/ml, VZV IgM negativ, Röteln IgG und IgM negativ, CMV IgG und IgM negativ. Der klinische Zustand des Kindes besserte sich unter Aciclovirtherapie über 21 Tage und supportiver Intensivtherapie (Katecholamine, Immunglobuline, Blutprodukte). Die Extubation war am Lebenstag 18 und die Entlassung nach Hause am Lebenstag 40 möglich. Der nun 5 Monate alte Junge hat sich altersentsprechend normal entwickelt und keine Reaktivierung der HSV-1-Infektion erlitten. Der HSV-1-IgG-Titer beträgt nun $490 \mathrm{AE} / \mathrm{ml}$, bei negativem IgM. Die Lymphozytensubpopulationen lagen innerhalb altersgematchter Referenzbereiche und es war immunphänotypsich kein Hinweis auf einen Immundefekt nachweisbar. 
Schlussfolgerung. Auch bei unauffälligem Vaginalbefund kann HSV übertragen werden und zu schweren disseminierten perinatalen HSVInfektionen führen. Im beschriebenen Fall ist sehr auffällig, dass die Mutter hohe IgG-AK-Titer gegen HSV und VZV aufwies und beim Neugeborenen keine (HSV) oder nur geringe (VZV) IgG-Titer gefunden wurden. Bei normalem Gesamt-IgG des Neugeborenen stellt sich die Frage, ob hier eine selektive plazentare Transportstörung für AK der Herpes-Gruppe vorlag.

\section{Posterwalk 9 - Ernährung}

\section{P09-01 \\ Häufigkeit und Charakteristika eines Dünndarmvolvulus ohne Malrotation bei Frühgeborenen (FG) $<1500 \mathrm{~g}$ in Deutschland - erste Daten einer ESPED-Erhebung}

\section{Y. Yarkin', A. Franz', C. Poets', C. Maas' \\ 'Universitätsklinik für Kinder- und Jugendmedizin Tübingen, Abt. Neonato- logie, Tübingen, Deutschland}

Hintergrund. Der Dünndarmvolvulus ohne Malrotation ist ein seltenes, jedoch potentiell lebensbedrohliches Ereignis, das sich v. a. bei Frühgeborenen (FG) initial nur schwer von anderen Krankheitsbildern mit akutem Abdomen unterscheiden lässt.

Fragestellung. Ziel dieser ESPED Erhebung ist die Erfassung der Inzidenz und potentieller Risikofaktoren bzw. frühzeitiger Warnsymptome dieses Krankheitsbildes bei FG $<1500$ g in Deutschland.

Methoden. Prospektive epidemiologische Studie. Datenerfassung seit 1.1.2014 anhand eines strukturierten Fragebogens über die Erhebungseinheit für seltene pädiatrische Erkrankungen in Deutschland (ESPED). Daten werden als Median und Spannweite angegeben.

Ergebnisse. 19 Fallmeldungen aus 2014 gingen ein, darunter 2 Falschmeldungen; 13 auswertbare Fragebögen liegen bislang vor. Das Gestationsalter bei Geburt (GA) betrug 25,7 (23,3-34,1) SSW. Das Geburtsgewicht lag bei 800 (490-1490) g. 8/13 betroffenen FG waren Mädchen. Das Lebensalter bei Manifestation betrug 36 (3-58) Tage, dies entspricht einem postmenstruellen Alter bei Manifestation von 31,56 (26,28-35,56) Wochen. Bei 2/13 FG kam es zu einer sehr frühen Manifestation am 3. bzw. 4. LT. Diese FG waren die reifsten der Kohorte (GA: 31,o bzw. 34,14 SSW) und zeigten intraoperativ eine anatomische Auffälligkeit (Ovarialzysten bzw. Ductus omphaloentericus). Bei einem dieser Kinder zeigten sich bereits im pränatalen Ultraschall dilatierte Darmschlingen Bei den anderen 11 FG manifestierte sich der Volvulus an LT 43 (13-58). Das postmenstruelle Alter bei Manifestation betrug $31,6(26,3-35,6)$ und das Geburtsgewicht 790 g (490-1150) [SDS: 0,01 (-1,18-0,76)]. 4/11 FG waren in den Tagen vor dem Ereignis mittels nasalem CPAP versorgt. 1/11 an der Highflow-Nasenbrille atemunterstützt. 2/11 waren spontan atmend und 4/11 über einen endotrachealen Tubus beatmet. Nasale IPPV und nasaler Hochfrequenz-CPAP kam bei keinem der Kinder zur Anwendung. Bisher konnten 7/13 FG ohne Kurzdarmsyndrom nach Hause entlassen werden. Bei 2 Kindern kam es zu einem Kurzdarmsyndrom, von 4 fehlen noch endgültige Daten zu diesen Fragen.

Schlussfolgerung. Ausgehend von ca. $7000 \mathrm{FG}<1500 \mathrm{~g}$ in Deutschland pro Jahr manifestiert sich ein Volvulus ohne Malrotation bei ca. 2-3/1000 FG $<1500$ g. $15 \%$ der betroffenen Kinder erleiden ein Kurzdarmsyndrom. Reifere FG scheinen häufiger anatomische Auffälligkeiten als Risikofaktor aufzuweisen, während die Entstehung bei den sehr unreifen FG weiterhin unklar ist. Um zufällige Schwankungen in Inzidenz und Klinik dieser seltenen Erkrankung ausschließen zu können, wurde die ESPED-Erhebung um ein weiteres Jahr verlängert. Wir danken den teilnehmenden Kliniken und bitten weiterhin um Ihre aktive Unterstützung.

\section{P09-02}

\section{Vergleich der Perzentilkurven der Geburtskörpermaße von Ein-} lingen, Zwillingen und Drillingen

M. Voigt ${ }^{1}$, N. Rochow ${ }^{2}$, H. Hagenah ${ }^{3}$, V. Hesse ${ }^{4}$, R. Hentschel, U. Wittwer-Backofen $^{6}$, D. Olbertz

'Inst. für Perinatale Wachstumsforschung, Sievershagen, Deutschland, ${ }^{2}$ Klinik für Allgemeine Kinder-und Jugendmedizin, Funktionsbereich Neonatologie/Intensivmedizin, Freiburg, Deutschland, ${ }^{3}$ Klinikum Rotenburg, Geburtshilfe, Rotenburg, Deutschland, 'Deutsches Zentrum für Wachstum, Entwicklung und Gesundheitsförderung im Kindes- und Jugendalter, Berlin, Deutschland, ${ }^{5}$ Institut/Klinik: Universitätsklinikum Freiburg, Zentrum für Kinder- und Jugendmedizin, Neonatologie/Intensivmedizin, Freiburg, Deutschland, ${ }^{6}$ Zentrum Medizin und Gesellschaft, Freiburg, Deutschland, ${ }^{7}$ Klinikum Südstadt Rostock, Abteilung für Neonatologie und Neonatologische Intensivmedizin/Kinderschlafmedizin, Rostock, Deutschland

Zielstellung. Es werden geschlechtsspezifische Perzentilkurven für die Körpermaße (Geburtsgewicht, Länge, Kopfumfang, längenbezogenes Geburtsgewicht) von Einlingen, Zwillingen und Drillingen vorgestellt und verglichen.

Material und Methode. Für die Jahre 2007-2011 standen uns Daten der deutschen Perinatalerhebung aus allen 16 Bundesländern mit $\mathrm{n}=3.187 .920$ Einlingen, $\mathrm{n}=110.313$ Zwillingen und $\mathrm{n}=369$ o Drillingen zur Verfügung. Die Daten erhielten wir vom AQUA-Institut in Göttingen. Die Berechnung der Perzentilwerte (3., 10., 25., 50., 75., 90., 97.) erfolgte mit dem Statistikprogrammpaket „SPSS“ mittels kumulativer Häufigkeiten. Es wurden manuelle Glättungen vorgenommen. Selektionen wurden nicht durchgeführt. Die Perzentilwerte für die Körpermaße der Einlinge und Zwillinge wurden bereits in der Zeitschrift „Geburtsh Neonatatol" 2014 publiziert.

Ergebnisse. Die geschlechtsspezifischen Perzentilkurtven (10.,50., 90.) für das Geburtsgewicht, die Länge, den Kopfumfang und das längenbezogene Geburtsgewicht von Einlingen, Zwillingen und Drillingen werden im direkten Vergleich präsentiert. Im Kurvenverlauf zeigen sich ganz deutliche Unterschiede zwischen Einlingen, Zwillingen und Drillingen. Die Zwillingskurven fallen gegenüber den Einlingskurven ab 31 Schwangerschaftswochen (50.Perzentile) und die Drillingskurven gegenüber den Einlingskurven schon ab 28 SSW (50. Perzentile) ab. In der 10. Perzentile werden diese Differenzen etwas später und in der 90. Perzentile schon früher sichtbar. Die 50. Geburtsgewichtsperzentilwerte für 36 SSW betragen für Einlinge $2750 \mathrm{~g}$, für Zwillinge $2430 \mathrm{~g}$ und für Drillinge $2320 \mathrm{~g}$.

Schlussfolgerung. Erstmals werden gesamtdeutsche Perzentilwerte für die Geburtskörpermaße von Einlingen, Zwillingen und Drillingen verglichen. Die Normwerte bei Geburt unterscheiden sich deutlich. Eine ausführliche Diskussion zu den Konsequenzen aus klinischer Sicht ist für die Zeitschrift „Geburtsh Neonatol“vorgesehen.

\section{P09-03}

Gastric residuals in preterm infants as predictor of tolerance to early enteral feeds (GRIP TRIAL)

B. Singh', N. Rochow', L. Chessell', K. Cunningham', J. Wilson', P. Murthy', C. Fusch', S. Thomas

'McMaster Children Hospital, Hamilton, Kanada

Introduction. Evidence is inconsistent to support checking gastric residual volumes (GRv) in predicting feeding intolerance in preterm infants. Checking of gastric residual volume remains standard practice in guiding feeding advancement in several neonatal centers. We hypothesizes that this practice delays establishment of full enteral feeding with associated complications.

Objective. The effect on time to reach full feeds $(120 \mathrm{ml} / \mathrm{k} / \mathrm{day})$ of not checking gastric residual volume in advancing feeds in preterm infants. Methods. Single Centre, unmasked, parallel armed RCT. Study site: Neonatal intensive care unit at McMaster Children Hospital. Inclusion 
criteria: Infants recruited within 48 hours of birth with BW between 1500 grams to 2000 grams. Exclusion criteria: Major congenital malformations, asphyxia and BW $<3$ rd percentile. Randomization: Variable number blocks stratified by BW. Study Intervention: Gastric residual volume assessed only with bloody aspirates or with vomiting and abnormal abdominal examination. Control: Gastric residual volume assessed routinely with feeding advancement.

Results. 87 infants were enrolled. There was no difference in time to reach full feeds in both groups. There was no difference in episodes of feeding interruptions, incidence of sepsis, reaching BW, and $120 \%$ of BW between two groups. However, two infants in the control group developed NEC. Please see table 1 and 2 for results.

Conclusion. There were no adverse events noted. The time to achieve full enteral feeds was short in both groups. In clinical practice, VLBW babies take longer time to reach full enteral feeds and gastric residuals could be a significant barrier to advancement of feeds. This study serves as a feasibility trial to do multicenter RCT to study effect of not checking gastric residual on time to reach full feeds and incidence of NEC in VLBW babies.

Acknowledgements. We wish to acknowledge the contribution of medical and nursing staff at Neonatal Intensive Care Unit at McMaster Children Hospital to conduct the study.

\section{P09-04}

\section{Application of fortifier during breastfeeding - a new perspective for the discharged premature infant?}

\section{Thanhäuser', A. Kreissl', S. Brandstetter', A. Repa', C. Binder', A. Berger', N. Haiden}

'Medizinische Universität Wien, Universitätsklinik für Kinder- und Jugendheilkunde, Abteilung für Neonatologie, pädiatrische Intensivmedizin und Neuropädiatrie, Wien, Österreich

Background. To meet the nutritional needs of preterm infants and to establish adequate growth, multicomponent fortifiers are added to expressed human milk until term or in growth restriction up to 52 weeks of gestation according to the ESPGHAN guidelines. This is in conflict with direct breastfeeding. We established a method of feeding fortifier with finger feeder during breastfeeding and investigated the impact of this new method on weight gain in preterm born infants after discharge. Furthermore, acceptance and practicability of fortification with the finger feeder were evaluated.

Materials und methods. Infants born $<34$ weeks were included in this observational study. Before discharge mothers were trained by lactation consultants to feed fortifier with finger-feeder during breastfeeding. Therefore the fortifier was dissolved in $23 \mathrm{ml}$ of warm water. The mixture was drawn up in a syringe attached to a finger-feeder and injected slowly in the mouth corner of the infant during breastfeeding. Primary outcome of the study was weight gain; secondary outcomes were acceptance and practicability of this new method.

Results. In total, 25 infants were analysed and divided into "fortifier acceptors" ( $\mathrm{n}=17)$ and "non-fortifier acceptors" $(\mathrm{n}=8)$. Demographic parameters were similar between the two groups. Weight gain per day after discharge was higher in the fortifier acceptors (median weight gain: 35,3 $\mathrm{g} / \mathrm{d}$ vs. $36,4 \mathrm{~g} / \mathrm{d}, \mathrm{p}=0,754)$ without reaching statistical significance. In $52 \%$ of the study population, the acceptance was very high; the other half reported feeding problems and irritation of the infant due to fingerfeeder use.

Discussion. Finger-feeder use for fortifier application in preterm infants enables mothers to exclusively breastfeed their baby. Weight gain of premature infants after discharge was higher in the group where mothers used this new method.

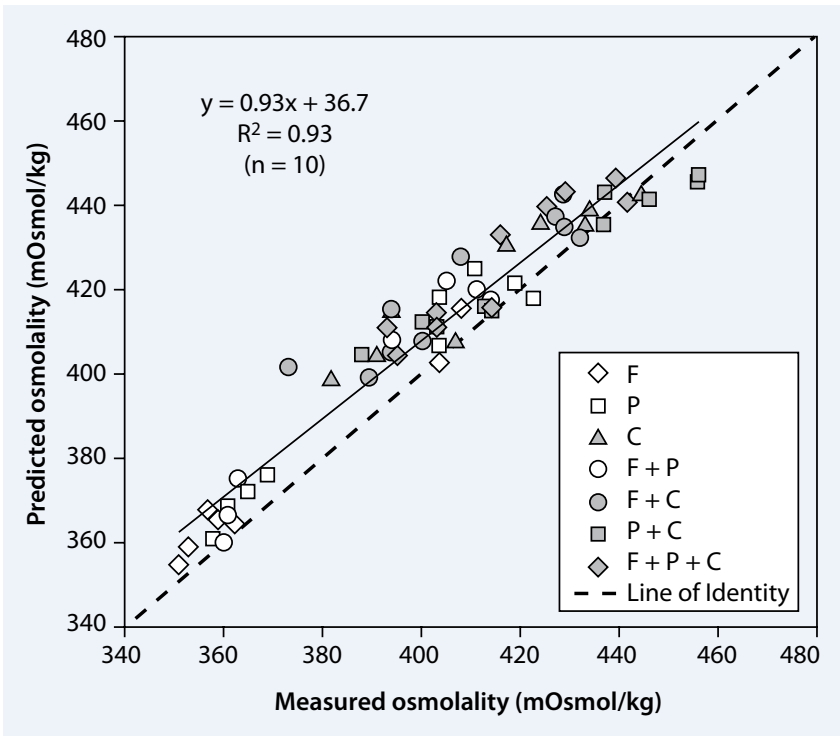

Abb. P09-05

\section{P09-05 \\ Can osmolality of target fortified breast milk for preterm infants be predicted?}

A. Choi', G. Fusch', N. Rochow', C. Fusch'

${ }^{1}$ Pediatrics, McMaster University, Hamilton, Jordan

Background. Breast milk (BM) is the best source of nutrition for preterm infants. Due to inter and intra-individual macronutrient variations in $\mathrm{BM}$, target fortification of $\mathrm{BM}$ (TFO) might become a desirable strategy to reduce macronutrient variations. However, fortifying BM with standard milk fortifier and additional macronutrients might increase osmolality. High osmolality has been implicated as potential risk factor of feeding intolerance and NEC. Thus, it would be beneficial if osmolality of fortified BM could be predicted prior to milk preparation.

Objective. 1) To establish and validate an osmolality prediction model within clinically relevant ranges for each macronutrient 2) To test feasibility using TFO samples for a post-validation in a clinical setting 3 ) To test change in osmolality after $24 \mathrm{~h}$ storage at $4^{\circ} \mathrm{C}$

Methods. 1) Prediction model: samples of pooled breast milk $(n=10)$ was gradually fortified with Polycose (P: 0.5-2.0 g), Beneprotein (B: 0.22.0 g), Microlipid (M: 0.5-4.0 g), and Aptamil protein (A: 0.2-2.0 g). A best-fit equation was generated for each component. Validation: fortified $\mathrm{BM}$ with each macronutrient $(\mathrm{n}=5)$ and combinations of macronutrients $(n=10)$ were tested in a set with separate samples. 2) Post-validation: TFO samples $(\mathrm{n}=700)$ were tested. 3$) \mathrm{BM}$ samples with $\mathrm{P}$ were stored at $4^{\circ} \mathrm{C}$ for $24 \mathrm{~h}$.

Results. 1) Prediction: linear correlation $\left(\mathrm{R}_{2}=0.89, \mathrm{R}_{2}=0.65, \mathrm{R}_{2}=0.98\right.$ for $\mathrm{P}, \mathrm{B}, \mathrm{A}$, respectively). Validation: linear correlation between measured and predicted osmolality values $\left[\left(\mathrm{R}_{2}=0.76, \mathrm{R}_{2}=0.82, \mathrm{R}_{2}=0.99\right.\right.$ for each $\mathrm{P}, \mathrm{B}, \mathrm{A}$, respectively) and $\left(\mathrm{R}_{2}=0.93\right.$ for combinations of macronutrients; Abb. Po9-05)]. 2) Predicted and measured osmolality values for TFO samples were in agreement with a precision of $\pm 10 \mathrm{mOsmol} / \mathrm{kg} .3) 24 \mathrm{~h}$ storage led to increase of average $20 \mathrm{mOsmol} / \mathrm{kg}$.

Conclusions. These models can predict osmolality of each macronutrient, combinations of macronutrients, and TFO samples and are applicable for these products above. It may be utilized as a simple quality assurance tool prior to feeding of preterm infants. 


\section{P09-06}

\section{Preterm single donor milk from mothers of preterm infants to} start enteral feedings in very low birth weight infants

A. Kreissl', E. Sauerzapf', A. Repa', C. Binder', M. Thanhäuser', B. Jilma², A. Berger', N. Haiden $^{\dagger}$

'Universitätsklinik für Kinder- und Jugendheilkunde, Medizinische Universität Wien, Abteilung für Neonatologie, pädiatrische Intensivmedizin und Neuropädiatrie, Wien, Österreich, ${ }^{2}$ Universitätsklinik für Klinische Pharmakologie, Medizinische Universität Wien, Wien, Österreich

Background. In premature infants human milk feedings are associated with a reduction of necrotizing enterocolitis (NEC), retinopathy of prematurity (ROP) and sepsis. During the first days of life breast milk is often not available and donor milk or preterm formula is recommended instead. Donor milk is always pooled and the nutritional properties are poor.

Objectives. Therefore, we implemented preterm single donor milk in our hospital and investigated the effect on time to full enteral feedings, growth and morbidity.

Material and methods. In a prospective study infants with a birth weight $<1500 \mathrm{~g}$ and a gestational age $<32$ weeks were fed with preterm single donor milk/breast milk until they were on full enteral feedings (PSDM group). These infants were compared with a historic control group receiving preterm formula/breast milk instead (Formula group). Preterm single donor milk was collected from mothers of preterm infants who donated for others.

Results. In total, 300 infants were analysed (150/group) and stratified according to birth weight in $<1001$ and $>1000$ grams. Time to full enteral feedings was shorter in the PSDM group ( 27 vs. 22 days $<1001 \mathrm{~g}$, n.s and 17 vs. 15 days $>1000 \mathrm{~g}, \mathrm{p}=0.01)$. The incidence of ROP ( $21 \%$ Formula group versus $7 \%$ PSDM group) and culture proven sepsis (37\% Formula group versus $13 \%$ PSDM group) was significantly lower in the PSDM group ( $\mathrm{p}<0.001$ for both parameters). There was no difference in gastrointestinal tolerance, weight gain and growth.

Conclusions. Feeding PSDM shortens time to full enteral feedings and reduces the incidence of ROP and culture proven sepsis especially in infants with a birth weight $<1001$ grams. Therefore, donor milk feeding and the concept "mothers of preemies donate for other preemies" should be encouraged in all NICUs.

\section{P09-07 \\ Enteral uptake of amniotic fluid, a major predictor for fetal growth during late gestation}

S. Bagci', C. Wije ${ }^{2}$, N. Roeleveld ${ }^{2}$, I. van Rooij', E. Brosen ${ }^{3}$, D. Tibboel', A. de Klein ${ }^{5}$, V. Choinitzki', J. Schumacher ${ }^{6}$, N. Zwink', E. Jenetzky', D. Katzer', J. Arand ${ }^{8}$, A. Mueller', P. Bartmann', H. Reutter

'Children's Hospital, University of Bonn, Department of Neonatology and Pediatric Intensive Care, Bonn, Deutschland, ${ }^{2}$ Radboud University Nijmegen Medical Center, Department of Epidemiology, Biostatistics and HTA ( $\left.{ }^{133}\right)$, Nijmegen, Niederlande, ${ }^{3}$ Erasmus Medical Centre,, Department of Clinical Genetics, Rotterdam, Niederlande, ${ }^{4}$ Erasmus Medical Centre Sophia Children's Hospital, Department of Pediatric Surgery, Rotterdam, Niederlande, ${ }^{5}$ Erasmus Medical Centre, Department of Clinical Genetics, Rotterdam, Niederlande, ${ }^{6}$ Institute of Human Genetics, University of Bonn, Bonn, Deutschland, ${ }^{7}$ German Cancer Research Center, Division of Clinical Epidemiology and Aging Research, Heidelberg, Deutschland, ${ }^{8}$ University Children's Hospital of Tübingen, University of Tübingen, Department of Neonatology, Tübingen, Deutschland

Background. There is good clinical evidence that in late gestation, normal fetal growth depends on a functioning fetal gastrointestinal tract to allow swallowing of amniotic fluid (AF) and nutritional uptake. There are certain lines of evidence that the inability of the fetus to swallow and/or absorb AF may lead to fetal growth retardation.
Aim. To investigate the importance of enteral uptake of AF for fetal growth during late gestation.

Materials and methods. We converted birth weight to z-scores (according to a European reference population) for each of 523 patients in a European cohort with esophageal atresia (EA) type I-III according to Vogt, excluding patients with H-type fistula. In order to account for the influence of the intestinal atresia on fetal growth per se, we also customized birth weight of each patient in a cohort of 504 patients with anorectal malformations (ARM) to the same European reference population. The data are expressed as median and interquartile range.

Results. Analysis of the complete cohort of EA and ARM patients showed: i) a significantly higher rate of SGA newborns with EA compared to ARM patients $(\mathrm{p}<0.001)$; ii) significantly lower birth weight $\mathrm{z}$-scores of EA compared to ARM newborns $(\mathrm{p}<0.001)$. Furthermore separate comparison between EA and ARM patients for term (gestational age (GA) $\geq 37$ weeks) and preterm (GA $<37$ weeks) newborns showed that $\mathrm{GA}$, birth weight, and z-scores of EA term newborns were significantly lower and rate of SGA of EA term newborns was significantly higher than those of ARM term newborns ( $p=0.002$ for GA and $p<0.001$ for others). Comparison of preterm EA and ARM newborns showed no difference for GA and SGA, while birth weight and z-scores of premature newborns were significantly lower for EA compared to ARM newborns ( $\mathrm{p}=0.002$ and $\mathrm{p}=0.004$, respectively). Z-scores of EA patients were significantly lower in term compared to preterm newborns with an inverse correlation with GA (Spearman correlation coefficient, $r=-0.171$, $\mathrm{p}<0.001$ ), whereas a positive correlation was demonstrated between GA and $\mathrm{z}$-scores for term and preterm ARM patients $(\mathrm{r}=0.104, \mathrm{p}=0.020)$. Conclusion. These results suggest that enteral uptake of AF by an intact fetal gastrointestinal tract may play a pivotal role in fetal growth and development especially during late gestation.

\section{P09-08}

\section{Muttermilch vs. Formula in der Ernährung Frühgeborener -} Outcome im Alter von ein und zwei Jahren

\section{Kirchner', N. Klambauer', R. Fuiko', S. Brandstetter'}

'Universitätsklinik für Kinder- und Jugendheilkunde, Klinische Abteilung für Neonatologie, Intensivmedizin und Neuropädiatrie, Wien, Österreich

Hintergrund. Es ist heute unumstritten, dass Muttermilch sowohl für reifgeborene als auch für frühgeborene Kinder die gesündeste Ernährung darstellt.

Fragestellung. Diese retrospektive Studie untersucht, ob es zwischen mit Muttermilch ernährten und mit Formula ernährten Frühgeborenen einen Unterschied in Bezug auf die motorische und intellektuelle Entwicklung mit ein und zwei Jahren sowie auf die anthropometrischen Daten mit einem Jahr gibt.

Patienten und Methoden. Im Studienzeitraum zwischen 01.01.200o und 31.12.2005 wurden 206 Frühgeborene mit einem Geburtsgewicht $<1500 \mathrm{~g}$ und/oder einem Gestationsalter $<29+6$ SSW retrospektiv erfasst und in zwei Gruppen aufgeteilt: 111 Frühgeborene mit Muttermilchernährung bzw. 95 Frühgeborene mit Formulaernährung in den ersten sechs Lebensmonaten. Im Alter von ein und zwei Jahren wurden die Frühgeborenen beider Gruppen hinsichtlich ihrer motorischen und intellektuellen Entwicklung (MDI = Mental- bzw. PDI = Psychomotor developmental index) verglichen. Ferner wurden die anthropometrischen Daten (Körpergewicht und Kopfumfang) mit einem Jahr zwischen den beiden Gruppen verglichen. Die Daten wurden aus den Vermont-Oxford-Erhebungsbögen und aus den Nachsorgemappen der Patienten entnommen. Als altersentsprechend galten sowohl beim MDI als auch beim PDI Werte über 84 . Alle Werte unter 85 wurden als mental bzw. psychomotorisch beeinträchtigt gewertet. Die statistische Auswertung der erhobenen Daten erfolgte mit Hilfe des Programms SPSS 22.0 für Windows.

Ergebnisse. Im Alter von einem Jahr zeigten in der Muttermilchgruppe $72,1 \%$ einen altersentsprechenden MDI, in der Formulagruppe nur $45,3 \%(\mathrm{p}=\mathrm{o}, 000)$. Im Alter von zwei Jahren hatten in der Muttermilch- 
gruppe $66,0 \%$, in der Formulagruppe nur $48,4 \%$ altersentsprechende MDI-Werte $(\mathrm{p}<0,05)$. Der PDI ergab im Alter von einem Jahr in der Muttermilchgruppe bei $61,3 \%$, in der Formulagruppe nur bei $38,3 \%$ altersentsprechende Ergebnisse $(\mathrm{p}=0,000)$. Im Alter von zwei Jahren zeigten $65,0 \%$ der Kinder in der Muttermilchgruppe und 46,2\% in der Formulagruppe altersentsprechende PDI-Werte $(\mathrm{p}=0,006)$. Die anthropometrischen Daten mit einem Jahr ergaben keinen signifikanten Unterschied zwischen den beiden Gruppen.

Schlussfolgerung. Frühgeborene, die in den ersten Lebensmonaten mit Muttermilch ernährt werden, zeigen eine bessere motorische und intellektuelle Entwicklung als Formula-ernährte Frühgeborene. In Bezug auf die körperliche Entwicklung im ersten Jahr konnte kein signifikanter Unterschied festgestellt werden.

\section{P09-09}

\section{Vitamin A administration using nasogastric tubes}

\section{S. Meyer', L. Gortner', D. Nohr', E. Maurer', D. Monz ${ }^{2}$, H. Biesalski ${ }^{3}$}

'Universitätsklinikum des Saarlandes, Klinik für Allgemeine Pädiatrie und Neonatologie, Homburg, Deutschland, ${ }^{2}$ Unversitätsklinik für Kinder und Jugendmedizin Gebäude ${ }^{9}$, Homburg, Deutschland, ${ }^{3}$ Universität Hohenheim, Stuttgart, Deutschland, ${ }^{4}$ Institut für Pharmakologie, Toxikologie und Experimentelle Pharmakotoxikologie, Homburg, Deutschland

Background. Administering oral medications to very small neonates using nasogastric tube (NGT) is often necessary because of their inability to swallow. When preparing drugs for NGT administration, the following key factors should be considered: adsorption by NGT, tube obstruction, the diluent and the volume of diluent used. To the best of our knowledge, no specific information is available in published form with regard to vitamin A application - an important growth factor in lung development and surfactant production - through an NGT.

Methods. In this experiment, 5 droplets of vitamin A solution (Vitadral', Aristo Pharma $\mathrm{GmbH}$, Germany; $1 \mathrm{ml}$ [approximately 27 droplets $=30.2$ $\mathrm{mg}$ retinol palmitate (54900 I.E.)] were mixed in $5 \mathrm{ml}$ of preterm formula (Prematil H, Milupa, Germany), and were then given slowly via a NGT (size: $\mathrm{CH} 4,5 \varnothing_{1,0 \times 1,5} \mathrm{~mm}$; Fa. Braun) over a 5-minute period. After passage through the NGT (a total of 6 runs and 6 measurements using a new NGT for each individual run), concentrations of retinol palmitate were measured. The same measurements were performed with 10 droplets of vitamin A mixed in $10 \mathrm{ml}$ of the same preterm formula feeds over a 10-minute period. These measurements were then compared to 5 and 10 droplets of vitamin A in $5 \mathrm{ml}$ and $10 \mathrm{ml}$ of preterm formula feeds without prior passage through a NGT (3 runs each). All measurements were performed in preterm formula after a run through the NGT and not in a blood sample. Retinyl palmitate was extracted from formula and oil samples (10o $\mu \mathrm{l} \mathrm{each)}$ by adding tetrahydrofuran (THF; $1,9 \mathrm{ml}$ ), vortexing and centrifugation. HPLC buffer (80\% acetonitril; $20 \%$ THF; $2 \mathrm{mg}$ butylhydroxytoluol as antioxidant) was added to supernatant of milk samples $(1,4 \mathrm{ml})$ and oil samples $(600 \mu \mathrm{l})$. Analysis was performed using standard HPLC method using UV-detection at $325 \mathrm{~nm}$.

Results. The measurements were as follows: 5 droplets of vitamin A in $5 \mathrm{ml}$ of feeds: $839 \pm 85 \mu \mathrm{M}$; 10 droplets of vitamin A in $10 \mathrm{ml}$ of feeds: $914 \pm 79 \mu \mathrm{M} ; 5$ droplets of vitamin A in $5 \mathrm{ml}$ of preterm formula without prior passage through NGT: $841 \pm 73 \mu \mathrm{M}$, and 1o droplets of vitamin A in $10 \mathrm{ml}$ of preterm feeds $946 \pm 34 \mu \mathrm{M}$. No significant differences were seen between the 3 measurements ( $\mathrm{p}>0.05$ ).

Conclusions. We conclude that vitamin A can be given as an oral solution with preterm formula feeds through a NGT, yielding similar concentrations when compared to oral administration. This finding may be of relevance to the conceptualization of future studies in the field of neonatology and pediatrics using vitamin A preparations.
P09-10

Wachstum von extrem unreifen Frühgeborenen (FG) nach schnellem Nahrungsaufbau

C. Maas', S. von Krogh', A. Bialkowski', C. Poets', A. Franz ${ }^{1}$

'Eberhard-Karls-Universität Universitätsklinik für Kinderheilkunde und Jugendmedizin, Neonatologie, Tübingen, Deutschland

Hintergrund und Fragestellung. Wir konnten zeigen, dass der enterale Nahrungsaufbau auch bei FG $<28$ SSW innerhalb von 7-10 $\mathrm{d}$ abgeschlossen werden kann (Maas et al., Neonatology 2013;103:67-73). Allerdings ging der raschere enterale Nahrungsaufbau mit einer signifikant geringeren parenteralen Eiweißzufuhr in der ersten Lebenswoche einher und war mit schlechterem Kopfwachstum assoziiert. Unter der Hypothese, dass die enterale Eiweißresorption bei diesen FG in den ersten Lebenstagen eingeschränkt ist, wurde das Ernährungskonzept für die ersten Lebenstage geändert, um eine höhere parenterale Eiweißzufuhr zu erzielen. Diese Studie soll prüfen, ob diese Veränderung zu einer Verbesserung der frühen postnatalen Gewichtsentwicklung und des Kopfwachstums von extrem unreifen Frühgeborenen führte.

Patienten und Methoden. Retrospektive Kohortenstudie. Eingeschlossen wurden $\mathrm{FG}<28$ SSW bei Geburt, die zwischen 1.1.2011 und 31.12.2013 geboren wurden und mindestens in den ersten 28 Lebenstagen in unserer Klinik behandelt wurden. Kohorte 1 (FG geboren zwischen 1.1.2011 und 30.9.2012) wurden nach dem ursprünglichen Ernährungskonzept behandelt, FG aus Kohorte 2 (geb. 1.10.2012 bis 31.12.2013) erhielten unabhängig von der enteralen Eiweißzufuhr in den ersten 3 Tagen (d) mind. $3 \mathrm{~g} / \mathrm{kg} / \mathrm{d}$ Aminosäuren intravenös (d 4 mind. $2 \mathrm{~g} / \mathrm{kg} / \mathrm{d}, \mathrm{d} 5$ mind. 1,5). Daten werden als Median (p25/p75) angegeben. Vergleiche zwischen Kohorten erfolgten mit dem Wilcoxon/Kruskal-Wallis-Test. Statistische Signifikanz war definiert als $\mathrm{p}<0,05$. Zur Beurteilung von Gewichtsentwicklung und Kopfwachstum wurde die SDS-Differenz zwischen d 28 und Geburt und zwischen Entlassung und Geburt berechnet (SDS-Berechnung mittels LMSgrowth, britische Referenzpopulation).

Ergebnisse. Es ergaben sich keine statistisch signifikanten Differenzen in Gewichtsentwicklung und Kopfwachstum bis d 28 oder Entlassung zwischen Kohorte 1 ( $\mathrm{n}=47$; Geburtsgewicht 740(650/950)g; Gestationsalter bei Geburt 26,57 $(25,43 / 27,43)$ SSW) und Kohorte 2 [n=30; $780(623-$ 980)g; 26,71(24,43-27,43)SSW]. Beide Kohorten erreichten bis zur Entlassung für Gewicht und Kopfumfang wieder ihre Geburtsperzentilen [SDS-Differenz Kopfumfang $0,28(-0,84 / 1,03)$ vs. $-0,09(-0,57 / 0,76)$, $\mathrm{p}=0,70$; SDS-Differenz Gewicht $-0,01(-0,58 / 0,49)$ vs. $-0,07(-0,54 / 0,37)$, $\mathrm{p}=0,73$ ] und erhielten bereits an $\mathrm{d} 7(6 / 16)$ bzw. $\mathrm{d} 5(4 / 7) 140 \mathrm{ml} / \mathrm{kg} / \mathrm{d}$ enteraler Nahrung $(\mathrm{p}=0,01)$.

Schlussfolgerung. Die zusätzliche parenterale Eiweißzufuhr führte im Kohortenvergleich nicht zu einer Verbesserung des Kopfwachstums, der potentielle Nutzen dieser Maßnahme sollte jedoch in einer größeren prospektiven, randomisierten Untersuchung geprüft werden. In beiden Kohorten wurde bei sehr unreifen FG ein dem intrauterinen Wachstum angenähertes frühes postnatales Wachstum erreicht.

\section{Posterwalk 10 - Patientensicherheit}

P10-01

\section{Retrospektive Analyse der TSH- und fT4-Werte einer 2-Jahres- Kohorte Frühgeborener (FG) $<32$ SSW}

A. Bialkowski', L. Schnaufer', J. Arand', A. Peter', G. Binder', C. Poets', A. Franz' 'Eberhard-Karls-Universität Universitätsklinik für Kinderheilkunde und Jugendmedizin, Neonatologie, Tübingen, Deutschland, ${ }^{2}$ Eberhard-KarlsUniversität Universitätsklinik für Kinderheilkunde und Jugendmedizin, Sektion Pädiatrische Endokrinologie, Tübingen, Deutschland

Hintergrund. Bei FG kann es zu transienten Hypothyreosen kommen, die unentdeckt und unbehandelt zu eingeschränkter neurokognitiver Entwicklung beitragen könnten. Richtlinien für eine Behandlung von 
FG mit L-Thyroxin (LT4) oder Iodid $\left(\mathrm{I}^{-}\right)$sind nicht etabliert. In unserer Klinik wurde 2004 festgelegt, dass bei FG $<32$ SSW zumindest an Tag 14 und bei Entlassung TSH und fT4 gemessen werden sollen, und dass bei isoliert erhöhtem TSH zunächst eine $\mathrm{I}^{--}$Substitution und bei vermindertem $\mathrm{fT}_{4}$ eine $\mathrm{LT}_{4}$-Substitution erfolgen soll.

Fragestellung. $\mathrm{Zu}$ prüfen, ob die lokale Praxis der Diagnose und Therapie von Schilddrüsenunterfunktion bei FG Kinder mit Verdacht auf Hypothyreose zuverlässig entdeckt.

Methode. Retrospektive Analyse der fT4 und TSH-Werte sowie der $\mathrm{I}^{-}$ und $\mathrm{LT}_{4}$-Substitution aller in unserer Klinik geborenen $\mathrm{FG}<32 \mathrm{SSW}$ der Jahre 2012-2013. Alle Daten sind als Median (Minimum-Maximum) dargestellt. Gruppenvergleiche erfolgten bei überwiegend nicht normal verteilten Daten mit dem Wilkoxon-Test.

Ergebnisse. In den Jahren 2012-2013 wurden 227 FG $<32$ SSW geboren und intensivmedizinisch versorgt. Gestationsalter bei Geburt: 293/7 (230/7-316/7) SSW, Geburtsgewicht 1215 (280-2260) g. 16 FG verstarben, davon 11 vor einer ersten TSH/fT4-Kontrolle. Bei 210 FG wurden an LT 14(9-28) erstmals TSH und $\mathrm{fT}_{4}$ bestimmt: TSH 3,85 (0,05-22,01) mU/l, fT4 16 (5-21) pmol/l. 26 FG (12,4\%) wurden daraufhin mit $\mathrm{I}^{-}$(Startdosis $25 \mu \mathrm{g})$ und $6(2,8 \%)$ mit L-Thyroxin behandelt [Startdosis: 4,25 (3,92$5,43) \mu \mathrm{g} / \mathrm{kg}$ ]. Im weiteren Verlauf wurden 7 weitere FG mit I- [ab Lebenstag 36,5 (24-63)] behandelt und 7 von $\mathrm{I}^{-}$auf LT4 umgestellt [LT 26 (2093)]. FG, die nach der ersten Kontrolle sofort mit LT 4 behandelt wurden, hatten die niedrigsten $\mathrm{fT}_{4}$-Werte, hier exemplarisch im Vergleich zu FG ohne I- oder LT4 Behandlung [7 (5-10) pmol/l vs. 16 (9-21) pmol/l; $\mathrm{p}<0,001]$. FG, die nach erster Kontrolle mit $\mathrm{I}^{-}$behandelt wurden hatten ein signifikant höheres TSH [Median 10,3 $(4,5-22,02) \mathrm{mU} / 1$ vs. 3,1 (o,578,51) $\mathrm{mU} / 1$; $\mathrm{p}<\mathrm{o}, \mathrm{oo1}$ ] als FG ohne I- oder $\mathrm{LT}_{4}$-Gaben, während die $\mathrm{fT}_{4}$ Werte ähnlich waren. FG mit LT4- bzw. I--Behandlung hatten jeweils signifikant niedrigere Gestationsalter bei Geburt als unbehandelte FG [251/7 (234/7 -271/7) bzw. 276/7 (243/7 -314/7); 303/7 (242/7-316/7) SSW, $\mathrm{p}<0,001$ für LT4 vs. keine Behandlung und $\mathrm{p}<0,001$ für $\mathrm{I}^{-}$vs. keine Behandlung]. Bei allen FG war das erste TSH-Screening unauffällig. Nur ein FG ( $\mathrm{I}^{-}$ab LT 13, dann L-T4 ab LT 24) zeigte ein auffälliges TSHScreening bei korrigiert 32 SSW.

Schlussfolgerung. Die hier untersuchten FG, v. a. diejenigen $<28$ SSW, zeigten $\mathrm{z}$. T. sehr niedrige fT4-Konzentrationen, die eine Routinekontrolle der SD-Werte (z. B. wie hier nach 14 d, vielleicht aber auch schon früher) sinnvoll erscheinen lassen. Diese retrospektive Analyse lässt keine Bewertung zu, welche Kinder von einer $\mathrm{I}^{-}$oder LT4-Gabe tatsächlich profitieren.

\section{P10-02 \\ Eine außergewöhnliche Ursache neonataler Krampfanfälle: vom Gehirn über das Herz zum NVK}

J. Kaiser', E. Salamano', K. Moser', J. Klepper², C. Wieg'

'Klinikum Aschaffenburg, Neonatologie und pädiatrische Intensivmedizin, Aschaffenburg, Deutschland, ${ }^{2}$ Klinikum Aschaffenburg, Kinderklinik, Aschaffenburg, Deutschland

Einleitung. Die Differentialdiagnosen von neonatalen Krampfanfällen sind vielfältig. Eine akribische Abklärung sollte unbedingt erfolgen. Kasuistik. Reifes weibliches Neugeborenes, Aufnahme am 10. Lebenstag, zuverlegt von einer auswärtigen Kinderklinik. Dort waren am 1. Lebenstag Neugeborenenkrampfanfälle mit generalisierten symmetrischen Kloni der Extremitäten aufgetreten. Primär dort antikonvulsiver Therapie mit Phenobarbital und Phenytoin, darunter zunächst Sistieren. Eine primäre Ursachensuche blieb ergebnislos. Nach 4 Tagen dann wiederholtes Auftreten von fokalen Krampfanfällen mit intermittierenden, spontan sistierenden Kloni im Bereich der rechten Körperhälfte, daraufhin Zuverlegung. Bei uns zunächst keine wegweisenden Befunde im EEG. Im Rahmen der Bildgebungen konnten, trotz wiederholter unauffälliger ZNS-Sonographien, im MRT-Schädel am 14. Lebenstag bilaterale subakute ischämische Infarkte im Versorgungsgebiet der A. cerebri post. bds. mit möglichem Befall der Sehrinde dargestellt werden. Bei V.a. embolische Streuung durch einen Primärthrombus wurde dieser gezielt via eine Echokardiographie und Sonographie der gr. Venen und Arterien gesucht. Im Bereich des Übergangs von der V. cava zum rechten Vorhof fand sich ein ca. $6 \mathrm{~mm}$ großer Thrombus bei Z. n. bei postnatal liegendem NVK. Eine umfangreiche ThrombophilieDiagnostik wurde eingeleitet. Es konnten neben einer 4GHomozygotie des PAI-Gens auch heterozygote Faktor-V-Leiden- Mutationen sowie des MTHFR-Gens nachgewiesen werden. Die antikonvulsive Dauertherapie wurde im weiteren Verlauf auf Levetiracetam umgestellt. Wie führten primär eine Antikoagulation mit niedermolekularem Heparin durch, darunter im Verlauf komplette Regredienz des Thrombus über 4 Wochen. In MRT-Verlaufskontrollen zeigten sich linksbetonte occipitale Parenchymdefekte.

Diskussion. Bei entsprechender Prädisposition ist in der Summe von einem deutlich erhöhten Thromboserisiko auszugehen. Bei noch offenem Foramen Ovale ist eine gekreuzte Embolie mit multiplen kleinen ZNS-Infarkte wahrscheinlich. Eine Thrombose der V. cava ist im Gegensatz zur Pfortaderthrombose nicht als NVK-assoziierte Komplikation typisch. Gekreuzte Embolien bei persistierendem Foramen ovale sind häufig mit einer schweren Morbidität wie cerebralen oder koronaren Ischämien assoziiert. Für die heterozygotze F-V-Leidenmutation sind neonatale cerebrale Infarkte gut beschrieben. Die Kombination einer Thrombose der VCI mit „neonatalem Stroke“ konnten wir in der Literatur nicht finden. In der Kombination mit anderen Thrombophiliefaktoren scheint ein zunehmendes Risiko zu bestehen.

Schlussfolgerung. Die Diagnostik bei cerebrale neonatale Infarkten sollte die Suche nach Systemthromben inkludieren. Bei einem thrombotischen Geschehen im Neugeborenenalter ist eine Thrombophiliediagnostik unbedingt indiziert.

\section{P10-03}

Hochrisikotransport Neugeborener zur ECMO-Therapie in Südbayern

M. Klemme', K. Förster', A. Schulze', S. Herber-Jonat', A. Flemmer'

'Dr. von Haunersches Kinderspital, Neonatologie der Kinderklinik am Perinatalzentrum Großhadern, München, Deutschland

Transporte Neugeborener mit akutem respiratorischem Versagen in ein ECMO-Zentrum sind durch eine deutlich erhöhte Mortalität während des Transportes gekennzeichnet. Folglich benötigt man zur Durchführung solcher Transporte ein hochspezialisiertes Team und eine hochspezialisierte technische Ausstattung. In Kooperation mit Stephan Medizintechnik wurde ein Transportsystem entwickelt, welches den hohen Anforderungen für den Transport schwerstkranker Neugeborener genügt. Das Transportsystem ist mit einem passiv erwärmten Inkubator, einem vollwertigen Patientenmonitoring und einem modernen Beatmungsgerät ausgestattet. Es stehen unterschiedliche patientengetriggerte, druck- und volumenkontrollierte Beatmungsmodi, Hochfrequenzoszillation und inhalatives Stickstoffmonoxid zur Verfügung. Das Beatmungsgas wird aktiv erwärmt und befeuchtet. Mit diesem Transportsystem wurden 28 Neugeborene mit schwerstem respiratorischem Versagen im sogenannten Prä-ECMO Zustand. (18/28 durch Hubschraubertransporte, EC-145). Alle Patienten wurden aus anderen Krankenhäusern abgeholt und durch einen erfahrenen Neonatologen und eine neonatologische Intensivschwester während des Transportes betreut. Der mittlere Transportweg betrug $72 \mathrm{~km}( \pm 49,5 \mathrm{~km}, \mathrm{SD}$; 8,4-175 km; min-max). Der mittlere Oxigenierungsindex (OI) war 37,3 (SD: 19,3; 5-100; min-max). Hochfrequenzoszillation wurde in $9 / 28$ (32\%) und inhalatives Stickstoffmonoxid in 19/28 (68\%) Neugeborenen eingesetzt (Mean iNO: 22,5 ppm, SD: 9,2 ppm; 5-40 ppm; min-max). Kein Patient verstarb während des Transportes. Insgesamt erhielten 15/28 (53\%) Patienten eine ECMO-Therapie nach Ankunft auf der Neugeborenintensivstation. 21 (75\%) Patienten überlebten und wurden nach Hause entlassen oder konnten zurückverlegt werden. Drei der 7 verstorbenen Patienten verstarben trotz ECMO-Therapie. Unser Transportsystem, welches höchsten technischen Anforderungen entspricht, kombiniert mit einem hochqualifizierten und speziell geschulten Team, 
stellt eine sichere und zuverlässige Option dar, Neugeborene mit respiratorischem Versagen oder anderen lebensbedrohlichen Erkrankungen zu transportieren.

\section{P10-04}

\section{Impact of paediatric simulation team training on the manage- ment of preterm infants}

\section{Wagner', K. Klebermass-Schrehof', F. Cardona', A. Berger', G. Schmölzer',} M. Olischar

'Department of Paediatrics and Adolescent Medicine, Medical University of Vienna, Austria, Division of Neonatology, Paediatric Intensive Care and Neuropaediatrics, Wien, Österreich, ${ }^{2}$ Department of Paediatrics, University of Alberta, Edmonton, Canada, Centre for the Studies of Asphyxia and Resuscitation, Neonatal Research Unit, Royal Alexandra Hospital, Edmonton, Canada, Edmonton, Kanada

Background and aim. Studies show that more than two thirds of perinatal death could be attributed to insufficient or ineffective team communication [1]. Therefore, it has been suggested to include simulationbased learning methods to acquire and enhance important skills for high-risk events such as neonatal resuscitation [2]. High fidelity simulation training is an ideal tool to improve team behavior [3]. We aim to examine if targeted simulation training improves skills, the postnata management of extremely preterm infants, teamwork and communication during simulated neonatal scenarios.

Methods. Physicians and nurses from several international hospitals are invited to our simulation centre. During a two-day workshop(WS), participants are exposed to different simulation scenarios. The WS includes delivery room management as well as less invasive surfactant administration. After a theoretical session, the participants are actively involved in simulation scenarios using the PremieHal ${ }^{\circ} / \mathrm{NewbornHal}^{\circ}$. All simulations are video recorded using SIMStation. The simulation room is fully equipped and resembles a Neonatal Intensive Care Unit (NICU). After each simulation, participants receive feedback using structured debriefing and video analysis. All participants have to complete a preand post-WS questionnaire. Follow-up-questionnaires after three and 12 months post-WS are sent by e-mail. These questionnaires are used to evaluate the clinical benefit of the training. The pre-WS questionnaire includes demographic data of participants (e.g. home institutional guidelines), current teamwork and communication during emergency situations in their NICU. Post-WS questionnaires assess their experience with the WS and their own learning effect. The follow-up questionnaires aim to determine whether the WS impacts the participants' approach of neonatal emergencies, teamwork, and communication or improves patient safety at the participants home NICU.

Results. This is an ongoing study. By comparing pre-, post- and followup questionnaires we can identify if the participants changed the management in their home hospitals and if these changes are related to a better outcome. Furthermore, we can identify what problems and obstacles occur during this process of change. The first analysis showed that our WS led to the introduction of regular simulation team trainings at the participant's hospital, to a change of teamwork and communication (e.g. identification of the team-leader) and to a better cooperation with the department of obstetrics and gynaecology. Also, participants wish to introduce special skills(e.g. developmental care handling procedures, less invasive surfactant administration procedure)in their NICUs.

Discussion. We employed a new approach of ongoing education and skills enhancement during the immediately newborn care. Global Rating Scales should be used for the objective measurement of teamwork before and after the WS. On-site visits after the WS for evaluating the benefit of the changes and to help introducing new methods locally would be preferable.

1. http://www.jointcommission.org/assets/1/18/SEA_30.PDF

2. Okuda Y et al (2009) The utility of simulation in medical education: what is the evidence?. Al Mt Sinai J Med

3. Thomas E J et al (2010) Team training in the neonatal resuscitation program for interns: teamwork and quality of resuscitations. Pediatrics

\section{P10-05}

Späte Auffälligkeiten im TSH-Screening (Topic: Patientensicherheit)

\section{J. Christoph', F. Guthmann}

${ }^{1}$ Kinderkrankenhaus auf der Bult, Neonatologie, Hannover, Deutschland

Hintergrund. Bei Frühgeborenen unter 32 Schwangerschaftswochen (SSW) soll gemäß den Kinder-Richtlinien ein abschließendes Zweitscreening in einem korrigierten Alter von 32 SSW erfolgen. Die GNPI empfiehlt mit Schreiben vom 13.11.2014 ein zusätzliches TSH-Screening bei Erreichen von 37 SSW bzw. vor Entlassung aus der Klinik.

Fragestellung. Diagnostizierten wir in den letzten 2,5 Jahren primäre Hypothyreosen zeitgerecht, wenn zusätzlich zu den G-BA-Vorgaben die GNPI-Empfehlungen maßgeblich gewesen wären?

Material und Methoden. Abfrage von TSH, $\mathrm{fT}_{4}$ und $\mathrm{fT}_{3}$ (Siemens Dimension EXL) in unserer Labor-EDV im Zeitraum von Juni 2012 bis Januar 2015 und Zusammenstellen mit zeitnahen TSH-Werten des Neugeborenen-Stoffwechselscreenings im Screeninglabor.

Tab. P10-05

\begin{tabular}{|c|c|c|c|c|c|c|}
\hline Gestationsalter (SSW) & Postnatales Alter (d) & fT4 (ng/dl) & $\mathrm{TSH}(\mathrm{mU} / \mathrm{l})$ & Postnatales Alter (d) & Screening-TSH (mU/l) & $\begin{array}{l}\text { Postnatales Alter bei } \\
\text { Therapiebeginn (d) }\end{array}$ \\
\hline 29 & 26 & 0,59 & 34,49 & 26 & 19,7 & 27 \\
\hline 25 & 67 & 0,97 & 14,07 & & & 101 \\
\hline 24 & 79 & 1,02 & 39,60 & 58 & $<2$ & 88 \\
\hline 25 & 90 & 0,60 & 87,79 & 90 & 58,8 & 93 \\
\hline 36 & 89 & 1,13 & 16,30 & 2 & 6 & 95, Trisomie 21 \\
\hline 37 & 113 & 1,32 & 17,28 & 12 & $<10$ & 113, Trisomie 21 \\
\hline 30 & 37 & 1,45 & 15,54 & 37 & 8,5 & \\
\hline 26 & 41 & 1,02 & 11,16 & 70 & $<2$ & \\
\hline 26 & 42 & 1,26 & 16,10 & 42 & 9,1 & \\
\hline 24 & 67 & 1,44 & 11,47 & 55 & 5,6 & \\
\hline 26 & 73 & 1,18 & 14,54 & 70 & 5,2 & \\
\hline 25 & 118 & 1,80 & 10,36 & 79 & 10,2 & \\
\hline 36 & 141 & 1,44 & 12,17 & 2 & 2,3 & 148 \\
\hline 36 & 153 & 1,52 & 11,38 & 3 & 17,6 & 9 \\
\hline
\end{tabular}


Tab. P10-06

\begin{tabular}{|lll}
\hline Parameter & 2013 & 2014 \\
\hline Anzahl (n) & 107 & 136 \\
\hline Gestationsalter (SSW) & $284 / 7( \pm 30 / 7)$ & $283 / 7( \pm 26 / 7)$ \\
\hline Geburtsgewicht (g) & $1031( \pm 283)$ & $1001( \pm 318)$ \\
\hline IVH $\left(\right.$ III $\left.{ }^{\circ}-\mathrm{IV}^{\circ}\right)$ & $15(14 \%)$ & $15(11 \%)$ \\
\hline BPD & $24(22,5 \%)$ & $32(23,5 \%)$ \\
\hline NEC & $9(8 \%)$ & $13(9,5 \%)$ \\
\hline
\end{tabular}

Ergebnisse. In 31 Monaten bestimmten wir Schilddrüsenfunktionswerte bei 453 Säuglingen im Alter von o bis 210 Tagen. 6 Neugeborene mit connataler Hypothyreose hatten TSH-Werte $>44 \mathrm{mU} / \mathrm{l}$ (im RoutineLabor) im Alter von 3 bis 6 Tagen. TSH-Werte oberhalb von $10 \mathrm{mU} / \mathrm{l}$ im Alter von 26 bis 153 Tagen hatten 14 Kinder, davon waren 10 bisher nicht auffällige Frühgeborene mit einem Gestationsalter unter 32 SSW. Eine L-Thyroxin-Therapie wurde eingeleitet bei 4 Frühgeborenen unter 32 SSW (Tab. P10-05, obere 4 Tabellenzeilen, 1-mal im Reifealter von 33 SSW, 3-mal in den letzten 3 Wochen vor errechnetem Geburtstermin) sowie bei 2 Säuglingen mit Trisomie 21 (nach dem 1. Lebensvierteljahr). Schlussfolgerung. Mit den G-BA- und GNPI-Vorgaben würden in unserer Patientengruppe alle Hypothyreosen Frühgeborener unter 32 SSW erfasst. Durch die unterschiedliche Matrix (getrocknetes Vollblut auf Screeningtestkarte versus Serum/Plasma im Routinelabor) differieren TSH-Werte derselben Blutentnahme teilweise um einen Faktor $\sim 2$. Die zusätzliche Bestimmung des freien Thyroxins kann der Einordnung/ Patientensicherheit dienen. Transiente TSH-Erhöhungen können einer Jodexposition (durch Röntgenkontrastmittel) folgen. Spätmanifestationen bei Down-Syndrom sind außerhalb der GNPI-Empfehlung zu erkennen.

\section{P10-06}

Neonatologisches Qualitätsmanagement im Rahmen einer Perinatalkonferenz nach GBA-Beschluss

\section{Mögel', A. Koch', J. Dinger', K. Nitzsche'2, M. Rüdiger'}

${ }^{1}$ Medizinische Fakultät Carl Gustav Carus an der TU, Fachbereich Neonatologie \& Pädiatrische Intensivmedizin, Dresden, Deutschland, ${ }^{2}$ Universitätsklinikum Carl Gustav Carus, Klinik und Poliklinik für Frauenheilkunde und Geburtshilfe, Dresden, Deutschland

Einleitung. Im GBA-Beschluss vom 20.06.2013 wird für Perinatalzentren eine Fallkonferenz für Frühgeborene $<1500 \mathrm{~g}$ (VLBW) gefordert diese sollte regelmäßig und strukturiert erfolgen. Bereits Hummler et al. konnten zeigen, dass durch Fallbesprechungen mit prospektivem Monitoring von Risikoindikatoren die Hirnblutungsrate reduziert und damit die Morbidität von kleinen Frühgeborenen gesenkt werden kann Methoden. Im Jahr 2014 wurde die Perinatalbesprechung entsprechend der GBA-Forderung modifiziert: seitdem wurden innerhalb von $7 \mathrm{~d}$ alle neuen Frühgeborenen $<1500$ g strukturiert in Anwesenheit von Kinderärzten, Kinderkrankenschwestern und Geburtshelfern besprochen. Dabei wurden Zielstellungen für das perinatale Management mit Fokussierung auf die IVH definiert (komplette ANS-Prophylaxe, Sectio im Frühdienst, Cord milking, Normothermie, INSURE, Vermeidung von Beatmung, Sedierung u. Katecholaminen u. a.).

Ergebnisse. 2014 wurden 136 Frühgeborene $<1500$ g am Perinatalzentrum Dresden versorgt. Davon konnten in 35 Perinatalkonferenzen 101 Frühgeborene (74\%) besprochen werden. Zur Perinatalbesprechung waren im Mittel 5 Pädiater, 1 Kinderkrankenschwester und 2 Geburtshelfer anwesend. Die mittlere Dauer der Fallkonferenz betrug 32 Minuten, im Mittel pro Frühgeborenen 13 Minuten. Die höhergradige IVH-Rate konnte etwas reduziert werden, die BPD- und die NEC-Rate blieben weitgehend konstant (Tab. P10-06). Die Rate des Cord milking als ein wichtiger Qualitätsparameter konnte im Verlauf des Jahres auf 88\% gesteigert werden.

Diskussion. Im Rahmen der Perinatalkonferenz konnte ein größerer Teil der VLBW interdisziplinär diskutiert werden. Im Vergleich zu den Ulmer Kollegen wurde die IVH-Rate durch die Fallbesprechungen bisher nur leicht gesenkt. Die Perinatalkonferenz wurde im Januar 2014 begonnen und verzeichnete im 2. Halbjahr eine Stabilisierung der Beteiligung der einzelnen Subdisziplinen. Ein wichtiges Ergebnis ist das gegenseitige Verständnis, welches nicht nur zu einer Verschiebung des Sectiozeitpunktes in den Frühdienst, sondern nahezu 90\% Cord-milking-Rate geführt hat.

Schlussfolgerung. Die geforderte Perinatalkonferenz ist ein Instrument des neonatologischen Qualitätsmanagements. Die Fortführung und Weiterentwicklung der interdisziplinären Zusammenarbeit mit Evaluation von Risikofaktoren für die perinatale Morbidität im Rahmen von Fallbesprechung ist sinnvoll und notwendig.

\section{P10-07}

\section{Kindernotarzt - professionelle Ergänzung im Rettungswesen}

M. Kuch

'Klinik für Kinder- und Jugendmedizin Karlsruhe, Karlsruhe, Deutschland

Kindernotfälle stellen mit ca. 1-3\% Anteil an Gesamtaufkommen der rettungsdienstlichen Einsätze eindeutig eine Minderheit dar. Im Umkehrschluss sind Kindernotfälle für die Mehrheit der Notärzte aber auch für das Rettungsdienstpersonal insgesamt, die am meisten gefürchtetsten Einsätze. Ursächlich dafür ist die niedrige Einsatzfrequenz und somit die fehlende Routine, andererseits ist die emotionale Belastung innerhalb des Notfallgeschehens extrem hoch. In der Praxis zeigte sich, dass bei entsprechend gelagerten pädiatrischen Notfällen das geschulte „Know-how“ eines speziellen Kindernotarztes gefragt ist. Sehr oft erfolgte der Ruf nach einem Spezialisten vor Ort zur Unterstützung des Notarztes im Nofallmanagement eines Kindernotfalls. Die Klinik hielt bis dato kein Personal für die präklinisch-pädiatrische Notfallversorgung vor. Zusätzlicher Zeitverlust entstand dadurch, dass der Spezialist erst von der Kinderklinik abgeholt und zu den jeweiligen Notfallorten gefahren werden musste. In Einzelfällen hat dies erheblichen Einfluss auf den Erfolg dieser Unternehmungen. Es ist klar, dass nur eine schnelle Alarmierung und das Vorhandensein eines eigenen Einsatzfahrzeuges die zeitlichen Voraussetzungen für ein effektives Handeln möglich machen. Aus diesen Überlegungen heraus erfolgte eine Kooperation des DRK Kreisverbandes Karlsruhe und der Kinderklinik Karlsruhe, um das Projekt eines Kindernotarzteinsatzfahrzeuges einzurichten. Neben der Grundkonzeption stellen wir im Vortrag das Team des Kindernotarztwagens Karlsruhe vor, gehen auf die speziellen Einsatzindikationen ein und werten ca. 1700 Notarzteinsätze aus. $\mathrm{Ne}$ ben Einsatzzeiten und Einsatzgebieten werden die hauptbetroffenen Altersgruppen der Patienten, das Geschlecht und die Schwere der Erkrankungen ausgewertet. Im Weiteren werden die akuten Erkrankungen, die zur notärztlichen Versorgung und stationären Aufnahme geführt haben ausgewertet und kindliche Traumaereignisse aufgearbeitet. Es erfolgt weiterhin eine Darstellung der geleisteten medizinischen Therapie (Zugänge, Intubationen, Monitoring etc.) sowie der medikamentö- 
sen Therapie. Zum Abschluss sollen folgende Schlussbemerkungen die Diskussion eröffnen:

1. Ein besonderes Rettungsmittel für Kinder fordert der Gesetzgeber nicht.

2. Bei einer Eisatzfrequenz von 17 Einsätzen im Monat versorgt der reguläre Notarzt durchschnittlich alle 1o Wochen einen pädiatrischen Notfall.

3. $70 \%$ der analysierten Kindernotfälle sind maßgeschneiderte Kindernotarzteinsätze (5\% Früh-Neugeborene und Geburten, 15\% Säuglinge, 49\% Kleinkinder bis zum Alter von 6 Lebensjahren).

4. Kinder haben einen Anspruch auf Versorgung durch speziell geschultes und spezialisiertes Fachpersonal in Arztpraxen, Kinderkliniken und Reha-Einrichtungen. Ist daraus zu folgern, dass sie auch in der Notfallsituation ein Anrecht darauf haben durch Spezialisten versorgt zu werden?

5. Der Kindernotarzt unterstützt konsiliarisch die regulären Rettungskräfte, d.h. der oft später eintreffende Kindernotarzt wird das erstversorgende Notarztteam unterstützen und evtl. die Versorgung und weitere Betreuung incl. Transport übernehmen. Er kann auch vor Ort die Funktion eines "Lehrnotarztes“ erfüllen, dient der Fortbildung am „kleinen Patienten“ für Notarzt und Rettungsdienstpersonal und letztendlich der Qualitätsverbesserung und Sicherung. Ist der Notfall durch den Regelrettungsdienst zu bewältigen, kann der Kindernotarzt abbestellt werden, er wäre für erneute Einsätze disponierbar.

\section{P10-08}

\section{Mehrsprachiger U2-Aufklärungsfilm: Gemeinschaftsprojekt der Hamburger Neonatologien}

\section{Singer ${ }^{1}$, C. Kutscha², A. Hennenberger ${ }^{3}$, L. Koch ${ }^{3}$, R. Laux ${ }^{4}$, S. Schmidtke, C. Schmitt' ${ }^{2}$ U. Thiede ${ }^{5}$, A. von der Wense ${ }^{6}$}

${ }^{1}$ Universitätsklinikum Hamburg-Eppendorf, Sektion Neonatologie und Pädiatrische Intensivmedizin, Hamburg, Deutschland, ${ }^{2}$ Helios Mariahilf Klinik, Hamburg, Deutschland, ${ }^{3}$ Katholisches Kinderkrankenhaus Wilhelmstift, Neonatologie und Pädiatrische Intensivmedizin, Hamburg, Deutschland, ${ }^{4}$ Asklepios-Klinik Barmbek, Neonatologie, Hamburg, Deutschland, ${ }^{5}$ Asklepios-Klinik Nord-Heidberg, Hamburg, Deutschland, ${ }^{6}$ Altonaer Kinderkrankenhaus, Neonatologie und Pädiatrische Intensivmedizin, Hamburg, Deutschland

Hintergrund und Zielsetzung. Besonders für Familien mit Migrationshintergrund und sprachlichen Problemen ist die Bedeutung der kinderärztlichen Neugeborenenuntersuchung $\mathrm{U}_{2}$ oft nicht ohne weiteres verständlich. Aber auch für deutschsprachige Eltern ist sie mitunter so überraschend oder „aufregend“, dass nicht alle Inhalte erfasst oder wichtige Fragen vergessen werden. Für umfassende Vorbereitungsgespräche steht im Klinikalltag jedoch nur begrenzte Zeit zur Verfügung. Durch einen im Intranet der Klinik abrufbaren Aufklärungsfilm soll daher allen Eltern die Möglichkeit gegeben werden, sich in aller Ruhe und in einer ihnen geläufigen Sprache auf die bevorstehende U2-Untersuchung ihres Neugeborenen vorzubereiten.

Material und Methoden. Die Idee des U2-Aufkärungsfilms wurde von einer lokalen gemeinnützigen Initiative (Hamburg macht Kinder gesund e.V.) gefördert und mit einer einschlägig erfahrenen Filmproduktionsgesellschaft (KS|MEDIA Produktionsgesellschaft für Film \& Content mbH, Hamburg; Frau H. Köhlert) realisiert. In Abstimmung zwischen allen Hamburger Neonatologien (Altonaer Kinderkrankenhaus, Asklepios Barmbek, Asklepios Nord-Heidberg, Helios Mariahilf, Katholisches Kinderkrankenhaus Wilhelmstift, Universitätsklinikum Eppendorf) wurden die Inhalte eines „neutralen Mittelteils“ erarbeitet, in dem der Ablauf der körperlichen Untersuchung „von Kopf bis Fuß“ - ergänzt um die Module Stoffwechselscreening, Hörtest, Pulsoximetrie und Hüftsonographie - dargestellt werden (Dauer 430"). Umgeben wird dieser gemeinsame Mittelteil von klinikspezifischen „bewegten Visitenkarten“, in denen sich die Kliniken mit ihrer individuellen „corporate identity" präsentieren, und die in Zusammenarbeit mit den jeweiligen Pressestellen gedreht wurden (Dauer 1' $44^{\prime}-25^{\prime} 5^{\prime \prime}$ ).
Ergebnisse und Diskussion. In einem ca. 15-monatigen Herstellungsprozess ist auf diese Weise ein Aufklärungsfilm entstanden, der den „idealtypischen“ Ablauf der U2-Untersuchung in leicht verständlicher Form wiedergibt und in sechs Sprachen (deutsch, englisch, französisch, türkisch, russisch, arabisch) zur Verfügung steht. Der Film ersetzt nicht die Aufklärung/Einwilligung (vor allem für das Stoffwechselscreening) im mediko-legalen Sinne, erlaubt aber auch solchen Familien eine angemessene Vorbereitung auf den ersten Kinderarztkontakt, die keinen Zugang zu entsprechenden Informationsmaterialien haben. Durch die Konstruktion aus einem „neutralen Mittelteil“ (in dem weder Örtlichkeiten noch handelnde Personen benannt werden) und „bewegten Visitenkarten" (in denen die jeweiligen Verantwortlichen in ihrem Arbeitsumfeld zu Wort kommen) lässt er sich mit geringem Aufwand an die Bedürfnisse jeder Klinik anpassen.

Schlussfolgerung. Das Projekt ist nicht zuletzt ein Beispiel für die mögliche fachliche Kooperation der neonatologischen Abteilungen einer Großstadt über die Grenzen der Kliniken bzw. Klinikträger hinweg.

\section{P10-09}

Simulation einer Meningokokken-Sepsis anhand eines „3D multitouch-Tisch-Tablet-PC": Ergebnisse einer randomisierten Interventionsstudie an Medizinstudierenden

M. Cremer', S. Buron', T. Harms'2, F. Behringer', U. von Zadow', H. Peters', K. Sostmann ${ }^{2}$

'Charité - Universitätsmedizin Berlin, Klinik für Neonatologie, Campus Mitte, Berlin, Deutschland, ${ }^{2}$ Charité - Universitätsmedizin Berlin, Dieter Scheffner Fachzentrum für medizinische Hochschullehre u. evidenzbasierte Ausbildung, Berlin, Deutschland, ${ }^{3}$ Technische Universität Dresden, Interactive Media Lab Dresden, Dresden, Deutschland

Hintergrund. Die Simulation von medizinischen Fällen anhand von virtuellen Patienten oder Patientensimulatoren ist in der Ausbildung akzeptiert und weit verbreitet. Hier stellen wir ein neuartiges Gerät (SimMed) vor, das insbesondere die Vermittlung von anwendungsbezogenen Lerninhalten verbessern soll: Es wurde Tisch-Tablet-PC entwickelt auf dessen 50-Zoll Bildschirmoberfläche ein 18-monate-altes Kleinkind dreidimensional darstellt wird. An diesem Kleinkind können verschiedene körperliche Untersuchungen (z. B. Inspektion der Haut, Auskultation von Herz und Lunge, Racheninspektion) durch die Bedienung der Touchscreen durchgeführt werden. Des Weiteren können diagnostische (z. B. Blutdruck messen, Sauerstoffsättigung, EKG, Blutgasanalyse, mikrobiologische und laborchemische Diagnostik) und therapeutische Maßnahmen (z. B. intravenöser Zugang, medikamentöse Therapie) angewendet werden.

Fragestellung. Verbessert eine Schulung am 3D-multitouch Tisch (SimMed) die Behandlung eines Kleinkindes mit Meningokokkensepsis am Modell im Vergleich zum herkömmlichen Unterricht (Computer-basiertes interaktives Fallszenario, virtueller Patient der Softwareapplikation $\left.\mathrm{CAMPUS}^{\star}\right)$ ?

Methoden. Alle 54 Studierenden des Reformstudiengangs Medizin des 6. Semesters wurden befragt, um freiwillig an der Studie teilzunehmen. 35 Studierende wurden eingeschlossen und in zwei Gruppen randomisiert: SimMed-Gruppe $(n=18)$ und CAMPUS-Gruppe $(n=17)$. Den Gruppen wurde eine typische Anamnese eines Kleinkindes mit Meningokokken-Sepsis präsentiert und die Studierenden hatten $20 \mathrm{Mi}-$ nuten Zeit, den virtuellen Patienten (SimMed bzw. CAMPUS) zu untersuchen, diagnostische Maßnahmen durchzuführen und entsprechend zu behandeln. Zwei Wochen später wurde ein 10-minütiger OSCE-Test (objective structured clinical examination) anhand einer Kleinkindpuppe mit den klinischen Zeichen einer Meningokokkensepsis durchgeführt. Im OSCE war gefordert, eine körperliche Untersuchung und diagnostische/therapeutische Maßnahmen durchzuführen. Anhand einer Checkliste mit 19 Items wurde geprüft (maximal zu erreichende Gesamtpunktzahl 38, o-2 Punkte je Item). Verglichen wurde die im OSCE erreichte Gesamtpunktzahl und die Punktzahl der einzelnen 


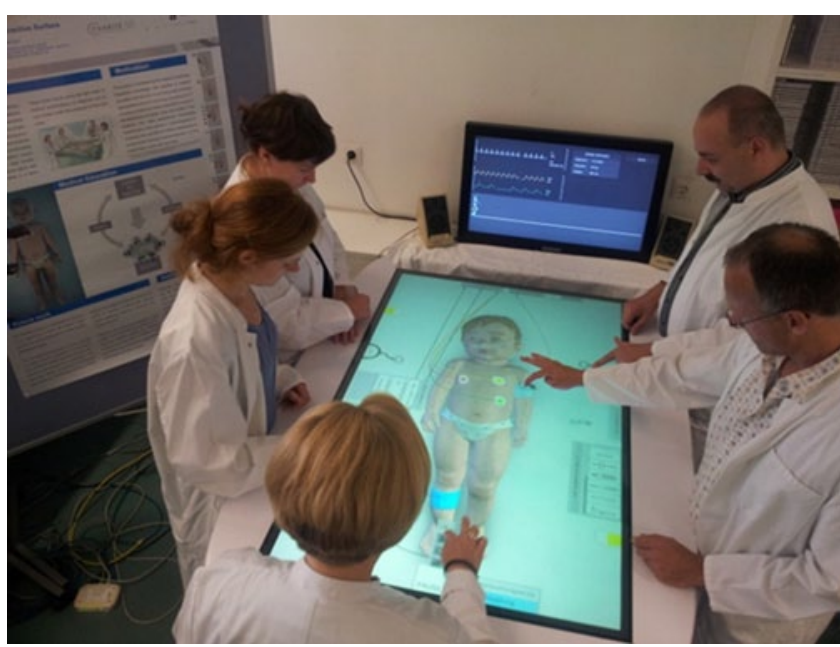

Abb. P10-09 SimMed-Tisch-Tablet in Benutzung 4

Items mittels t-test. Die OSCE-Prüfer waren hinsichtlich der Gruppenzuordnung geblindet.

Ergebnisse. Die SimMed-Gruppe erreichte im OSCE-Test signifikant mehr Gesamtpunkte (MW 29,4; $\mathrm{SD} \pm 4,3$ ) als die CAMPUS-Gruppe (MW 24,0; $\mathrm{SD} \pm 3,4 ; \mathrm{p}<0.001)$. Insbesondere bei den therapeutischen Maßnahmen wie Volumengabe und Kortikosteroidgabe erreichten die Studierenden der SimMed-Gruppe bessere Ergebnisse. Bei den diagnostischen Maßnahmen, wie Auskultation, Blutdruckmessen, Sauerstoffsättigung messen zeigte sich kein signifikanter Unterschied zwischen den Gruppen.

Diskussion. Die Ergebnisse dieser Studie zeigen, dass die Schulung am SimMed-Tisch eine geeignete Möglichkeit darstellt, das theoretisch erworbene Wissen in konkrete Handlungen bei einer Patienten-Simulation umzusetzen (Abb. P10-09).

\section{Posterwalk 11 - Pädiatrische Intensivmedizin}

\section{P11-01}

\section{Schwere Zynanidvergiftung im Rahmen einer alternativmedi- zinischen Behandlung mit Amydalin und Aprikosenkernen bei einem 4-jährigen Jungen}

\section{S. Meyer', S. Gottschling ${ }^{2}$, E. Tutdibi', L. Gortner ${ }^{3}$, I. Oster ${ }^{4}$}

'Universitätsklinikum des Saarlandes, Klinik für Allgemeine Pädiatrie und Neonatologie, Homburg, Deutschland, ${ }^{2}$ Universitätsklinikum des Saarlandes, Klinik für Kinder- und Jugendmedizin, Zentrum für Kinderschmerztherapie und Palliativmedizin, Homburg, Deutschland, ${ }^{3}$ Unversitätsklinik für Kinder und Jugendmedizin Gebäude ${ }^{9}$, Homburg, Deutschland, ${ }^{4}$ Universitätskinderklinik Homburg, Homburg, Deutschland

Hintergrund. Die Verwendung von Komplementär- und Alternativmedizin (CAM) findet bei pädiatrischen Patienten mit bösartigen Erkrankungen nicht selten statt.

Patienten und Methodik. Fallbericht.

Ergebnisse. Hier berichten wir über einen 4-jährigen Jungen mit einem metastasierten Ependymom, bei welchem es im Rahmen einer CAMBehandlung zu einer schweren iatrogenen Zyanidvergiftung gekommen war. Klinisch imponierte ein encephalopathisches Krankheitsbild mit Agitation und einer schweren initialen Bewusstseinsstörung (GCS: 3). Die bei Aufnahme durchgeführte Blutgasanalyse zeige eine schwere metabolische Azidose mit ausgeprägter Laktaterhöhung. Nach intensiver Befragung der Eltern berichteten diese über die Inanspruchnahme von CAM-Therapien. Hierbei wurden über einen Zeitraum von rund einer Woche tägliche Infusionstherapien sowie orale Gaben mit soge- nanntem „Vitamin B17“ durchgeführt. Nach Gabe von Natriumthiosulphat kam es zu einer raschen Besserung der klinischen Symptomatik ohne Auftreten von Residuen. Die toxikologische Bestimmung der Serumzyanidkonzentration ergab einen hochpathologischen Befund.

Schlussfolgerung. Bei Kindern, bei denen es zum Auftreten eines akuten encephalopatischen Krankheitsbildes kommt, sollte differenzialdiagnostisch an eine iatrogene Zyanidintoxikation gedacht werden. Diese kann im Rahmen einer CAM-Therapie mit Amydalin und Aprikosenkernen auftreten.

\section{P11-02}

Ausgeprägte Hyperphosphatämie und Hypocalcämie mit Tetanie und Krampfanfall nach akzidentieller Klistiergabe beim Säugling: Therapie und Konsequenzen

S.Trepels-Kottek',E.Verjans', G.Steinau', K.Heimann',M.Schoberer', T.Orlikowsky'

'Universitätsklinikum Aachen, Kinderklinik Sektion Neonatologie und Kinderintensivmedizin, Aachen, Deutschland, ${ }^{2}$ Med. Einrichtungen der RWTH Aachen, Chirurgische Universitäts- und Poliklinik der RWTH Aachen, Aachen, Deutschland

Hintergrund. Abführende Maßnahmen mittels Klysmen zur raschen Entleerung des Darms bei Obstipation und fehlender Stuhlpassage sind Routine. Phosphat als Hauptbestandteil kann durch Resorption eine Hyperphosphatämie bewirken. Calcium-Phosphat-Komplexbildung bedingt unterschiedlich ausgeprägte Hypocalcämien mit teils lebensbedrohlichen Komplikationen, wie Tetanien, Krampfanfällen und Asystolien.

Falldarstellung. 10 Monate alter, männlicher Säuglings mit bekanntem M. Hirschsprung, vorgestellt wegen Fieber $\left(39,9^{\circ} \mathrm{C}\right)$, schlechtem Trinkverhalten und abdomineller Distension. Mehrfache Darmoperationen, Rektum- und Sigmaresektion mit Kolostomaanlage, 3 Wochen zuvor Rückverlagerung. Ausreichender Hautturgor, regelrechter kardiopulmonalen Befund, pralles, druckschmerzhaftes Abdomen mit Hyperperistaltik. Radiologischer Ausschluss einer Nahtinsuffizienz. Bei V. a. Passagestörung abführende Maßnahmen mit Entleeren von viel Stuhl, danach gebessertem abdominellen Befund. 4 Stunden später Allgemeinzustandsverschlechterung, Hyperthermie $\left(42,0^{\circ} \mathrm{C}\right)$, Tachykardie (220/min), anstoßender Atmung und eingeschränkte Vigilanz. Verdacht auf septischen Schock daher Übernahme Kinderintensivstation. Apathisches Kind mit Pfötchenstellung beider Hände, Faszikulieren des Mundes, rudernden Bewegungen der Arme. BGA: BZ 406 mg/dl, $\mathrm{pH} 7,36, \mathrm{pCO} 22 \mathrm{mmHg}, \mathrm{BE}-11,3$, Natrium $152 \mathrm{mmol} / \mathrm{l}$ und Calcium++ o,4 mmol/l. Weitere Laborparameter: CRP $88,9 \mathrm{mg} / \mathrm{l}$, Kreatinin o,65 $\mathrm{mg} / \mathrm{dl}$, Procalcitonin 39,33 ng/ml, Phosphat $17 \mathrm{mmol} / \mathrm{l}$. QTc-Zeit $496 \mathrm{~ms}$. Therapie. Unter Monitorkontrolle Calciumglukonat 10\% bis zum Sistieren des Krampfanfalls und der Pfötchenstellung, weitere Infusion mit 2\%iger Calciumlösung. Insulintherapie, Cefuroxim und Metronidazol. $\mathrm{NaCl}$ 0,9\% und Sterofundin Iso Infusion, Antipyrese mit Paracetamol und Ibuprofen.Kontinuierlicher Anstieg Serum-Calciums, Normoglykämie, Normothermie, Ausgleich der hypertonen Dehydratation. Nach wenigen Stunden normalisiertes Serumphosphat, am ehesten durch die Insulintherapie begünstigt wegen Verschiebung des Phosphats in den intrazellulären Raum. Normalisierung Kreatinin, BGA und QTc-Zeit. Ergebnisse. Zur Unterbrechung von hypocalcämischen Krampfanfällen ist die rasche Calciumsubstitution unter Monitorkontrolle erforderlich. Zusätzlich muss eine Rehydratation begonnen werden und eine Hyperphosphatämie kann mittels Insulin/Glukose-Substitution positiv beeinflusst werden.

Fazit. Regelmäßiges Erörtern neuster Empfehlungen der Arzneimittelkommission. Altersentsprechende Kennzeichnung auch vermeintlich harmloser Medikamente. Bei Abführenden Maßnahmen Altersbegrenzung und Kontraindikationen, insbesondere gestörte Darmschleimhautbarriere beachten. Schwere Elektrolytentgleisungen müssen unter Reanimationsbereitschaft mit intensivmedizinischer Überwachung ausgeglichen werden. 


\section{P11-03}

Weniger Fälle, weniger Tote, weniger Traumata: Perspektiven der präklinischen Reanimation von Kindern

M. Schoberer', V. Rotering', S. Trepels-Kottek', K. Heimann', T. Orlikowsky' 'Uniklinik RWTH Aachen, Klinik für Kinder- und Jugendmedizin Sektion Neonatologie und Pädiatrische Intensivmedizin, Aachen, Deutschland

Hintergrund. Die präklinische Reanimation von Kindern zählt zu den einschneidendsten menschlichen und ärztlichen Herausforderungen überhaupt. Das Ereignis trifft Patienten, Angehörige und Therapeuten in der Regel unvorbereitet, es erfordert schnelles, zielgerichtetes Handeln unter stärkster emotionaler Belastung. Die Reanimation ist in der Regel erfolglos, konfrontiert aber auch bei Wiederherstellung der Vitalfunktionen alle Beteiligten mit existentiellen Fragen und Ängsten, für die es zunächst, und manchmal quälend lange keine Klarheit gibt. Einer erfolgreichen Reanimation schließt sich nicht selten eine intensivmedizinische Behandlung an, die über maximale Therapieintensität in eine Sterbebegleitung mündet. Die wohl quälendsten Fälle sind die durch Kindesmisshandlung sowie solche, bei denen die Reanimation zu einem vegetativen Status ohne Kommunikationsfähigkeit des Patienten führt.

Fragestellung. Wir möchten vor diesem belastenden Hintergrund eine Übersicht über Möglichkeiten zur Verringerung der Inzidenz, Verbesserung der Qualität und zur Individualisierung der Therapieintensität präklinischer Reanimationen darstellen.

Methoden. Wir zeigen Entwicklungen der Prävention von SIDS, Ertrinkungs- und Verkehrsunfällen, risikosuchendem Verhalten Jugendlicher sowie der Suizid-Prävention auf. Beispielhaft werden Präventionskampagnen auf der Basis neuer Medien vorgestellt. Ferner aktuelle Daten zum Einfluss der vereinfachten ERC-Guidelines auf die Laien-Compliance bei Reanimationen, Effekte der telefonassistierten Reanimation (T-CPR) und der zunehmenden Verbreitung von AED's sowie aktuelle Daten zur therapeutischen Hypothermie bei Kindern nach Herz-Kreislaufstillstand. Anhand eigener (s. a. eingereichtes Abstract: „Epidemiologie und Outcome präklinischer Reanimationen bei Kindern und Jugendlichen in Aachen 2003-2013: anders als erwartet") und publizierter Daten bewerten wir die Validität von Outcome-Prädiktoren sowie die Übertragbarkeit von Algorithmen des Therapieabbruchs aus der Erwachsenenreanimation (Termination of Reanimation, ALS-TOR).

Diskussion. Die Prävention präklinischer Reanimationen ist gerade vor dem Hintergrund des Outcomes die bei Weitem erfolgversprechendste Maßnahme zur Verringerung der Kindersterblichkeit jenseits der Neugeborenenperiode. Wir sehen Potential insbesondere in einer weiteren Verbesserung der SIDS-Prävention aber auch bei der Verbesserung des Risikobewusstseins für Ertrinkungsunfälle im Kleinkindalter. Neben der Themenauswahl sollten auch die Chancen der Nutzung neuer Medien zur Verbreitung von Präventionsprogrammen ausgelotet werden. Die Entwicklung im Bereich der Verkehrsunfälle ist erfreulich, wobei der Trend sich deutlich verlangsamt hat. Es bleibt abzuwarten, wie sich die Qualität der Kinderreanimationen verändert, wenn sich in der Erwachsenen-Laienreanimation der Verzicht auf eine Atemspende durchsetzt. Die Wertigkeit der therapeutischen Hypothermie in der Kindermedizin muss schnellstmöglich durch prospektive Multicenterstudien geklärt werden. Allgemeingültige Empfehlungen zur Verringerung der Therapieintensität anhand epidemiologisch basierter Algorithmen oder prädiktiver Variablen müssen mit zunehmender Verbreitung der therapeutischen Hypothermie neu adjustiert werden.

\section{P11-04}

Retrospektive Analyse von sonographischen Befunden bei Neugeborenen mit Infarkt der Arteria cerebri media im Zeitraum von 2000-2015

\section{Schlunk', M. Vochem ${ }^{2}$}

'Klinikum Stuttgart, Olgahospital Stuttgart, Pädiatrie IV, Neonatologie, Stuttgart, Deutschland, ${ }^{2}$ Olgahospital, Neonatologie, Stuttgart, Deutschland

Hintergrund. Rund ein Drittel aller kindlichen Schlaganfälle treten in der Neona talperiode auf [1]. In 70-90\% manifestiert sich der Infarkt mit einem generalisierten oder fokalen Krampfanfall [2]. Als Residuum besteht in bis $\mathrm{zu} 50 \%$ eine unilaterale spastische Hemiparese. In 10-15\% persistieren die Krampfanfälle. Teils kommt es zu kognitiven Defiziten und Entwicklungsverzögerungen [3]. Als diagnostischer Goldstandart gilt die Kernspintomographie, welche vor allem in der prognostischen Einschätzung des motorischen Outcomes eine wichtige Rolle spielt [4]. Die Schädelsonographie hat aber in der initialen Diagnosestellung, sowie bei Verlaufskontrollen einen großen Stellenwert. So steigt die Sensitivität von repetitiven Ultraschalluntersuchungen von initial $68 \%$ (Tag 1-3) auf 87\% (Tag 4-10) [5]. In einer retrospektiven Analyse aller stationären Neugeborenen im Zeitraum von 2000-2015 konnten $11 \mathrm{~Pa}-$ tienten mit einem Infarkt der Arteria cerebri media rekrutiert werden. Besonderes Augenmerk in der Auswertung galt unter anderem der Sensitivität repetitiver Ultraschalluntersuchungen im Vergleich zur Kernspintomographie, sowie der dopplersonographischer Befunde.

Ergebnisse. Elf Neugeborene konnten im oben genannten Zeitraum rekrutiert werden. Davon 7 Termingeborene $(37+1$ bis $41+0$ SSW) und 4 Frühgeborene ( $32+1$ bis $36+2$ SSW). 9 von 11 Neugeborenen wurden in den ersten 4 Lebenstagen symptomatisch. 6 Kinder (alle $>37$ SSW) wurden mit klinisch eindeutigem fokalem oder generalisiertem Krampfanfall manifest. Ein Termingeborenes fiel über ein Bewegungsdefizit der linken Extremität auf. Unspezifische $(n=2)$ bzw. keine Symptome $(n=2)$ zeigten sich ausschließlich bei Frühgeborenen. 9 von $11(81,8 \%)$ EEG Untersuchungen waren initial auffällig. 9 von 11 Kindern $(81,2 \%)$ erhielten das erste Schädelsonogramm am Tag des Symptombeginns. Die Diagnose konnte bei 7 von 11 Patienten $(63,6 \%)$ im ersten Ultraschall gestellt werden. Bei den verbleibenden 4 Kindern (36,5\%) wurde die Diagnose erst im Verlauf durch repetitive Untersuchungen gestellt (max. 2 Tage nach erster Schädelsonographie bzw. Symptombeginn). Die gepulste Dopplersonographie zeigte in 5 von 11 Kindern (45,5\%) auffällige Befunde. 7 von 11 Kindern erhielten eine Kernspintomographie des Neurokraniums, welche den sonographischen Befund jeweils bestätigte.

Schlussfolgerung. Die Schädelsonographie ist eine äußerst zuverlässige Methode in der Detektion von Hirninfarkten im Neugeborenenalter. Bei entsprechendem klinischem Verdacht sollte so früh wie möglich eine Schädelsonographie mit Doppleruntersuchung erfolgen. Ist die Erstuntersuchung unauffällig, so müssen repetitive Untersuchungen erfolgen. Damit steigt die Sensitivität nochmals deutlich an und ist vergleichbar mit der der Kernspintomographie. Inwiefern kleinere Läsionen übersehen und somit ggf. nicht der Kernspintomographie zugeführt werden bleibt offen.

1. Münster Schlaganfallregister

2. Kirton $A$ et al (2011) Pediatrics 128:e1402-10

3. Kirton A et al (2008) Ann Neurol 63:436-43

4. Mercuri E et al (2004) Pediatrics 113:95-100

5. Cowan F et al (2005) Arch Dis Child Fetal Neonatal Ed 90:F252-6 


\section{P11-05}

Hämodiafiltration bei Säuglingen und Kleinkindern mit terminalem Nierenversagen und Komplikationen der Peritonealdialyse

S. Rödl', I. Marschitz' ${ }^{2}$ R. Ulreich', C. Mache ${ }^{3}$, G. Zobel ${ }^{4}$

'Universitätsklinik für Kinder und Jugendheilkunde, Pädiatrische Intensivstation, Graz, Österreich, ${ }^{2}$ Neonatologie Salzburg, Salzburg, Österreich, ${ }^{3}$ Universitätsklinik für Kinder und Jugendheilkunde, Medizinische Universität Graz, Graz, Österreich, ${ }^{4}$ Universitätsklinik für Kinder- und Jugendheilkunde, Pädiatrische Intensivstation, Graz, Österreich

Hintergrund. Die Mortalität bei kleinen pädiatrischen Patienten mit terminalem Nierenversagen ist nach wie vor hoch.

Fragestellung. Verbesserung des Überlebens durch intermittierende oder kontinuierliche Hämodiafiltration (HDF) bei Säuglingen und Kleinkindern mit terminalem Nierenversagen und Komplikationen auf Peritonealdialyse (PD).

Patienten und Methoden. Wir zeigen die Therapie PD und HDF bei 11 Säuglingen und Kleinkindern bei terminalem Nierenversagen von 1995 bis 2015. Zum terminalen Nierenversagen führten folgende Diagnosen: kortikale Nekrose nach perinatalem Schock, beidseitige Nierendysplasie bei Prune-belly-Syndrom mit nachfolgender bilateraler Nephrektomie, neonatales Shigatoxin-assoziiertes hämolytisch-urämisches Syndrom mit bilateraler Nephrektomie und autosomal rezessive polyzystische Nierendysplasie mit bilateraler Nephrektomie, posteriore Urethralklappe, dysplastische Beckennieren und Denys-Drash-Syndrom.

Ergebnisse und Diskussion. Zunächst wurden 10/11 der Patienten mit HDF gestartet. Die Gründe für HDF waren eine ausgedehnte abdominelle chirurgische Interventionen, vermutetes akutes Nierenversagen und Kurzdarmsyndrom. Danach wurden 7 Patienten auf PD umgestellt Bei Komplikationen der PD wurden Patienten mit HDF behandelt Bei schwerer Hypervolämie begannen wir mit einer kontinuierlichen HDF und setzten die Behandlung nach Erreichen der Normovolämie mit intermittierender HDF fort. 6 der 7 der PD Patienten benötigten 1-3 HDF Rescue Behandlungsperioden (ausgedehnte abdominelle Chirurgie 7-mal und rezidivierende Peritonitis in 2 Fällen). 2 PD-Patienten mussten wegen multiplen retroperitonealen Hernien mit schwerer Filtrationsstörung und akut rezidivierender Pankreatitis mit Peritonitis dauerhaft auf HDF umgestellt werden. 1o Patienten überlebten das erste Lebensjahr, 1 Patient verstarb an dilatativer Kardiomyopathie. Derzeit wird ein Patienten im Alter von 14 Monaten mittels HDF und ein Patient im Alter von 11 Monaten mittels PD behandelt. 8 Patienten wurden erfolgreich im Alter von 1 bis 7 Jahren transplantiert.

Schlussfolgerung. Im Falle von Komplikationen bei PD ist die HDF die einzige Alternative um die Zeit bis zu einer Nierentransplantation zu überbrücken.

\section{P11-06}

\section{Epidemiologie und Outcome präklinischer Reanimationen in Aachen 2003-2013: Anders als erwartet!}

M. Schoberer', V. Rotering', J. Brokmann'2, S. Beckers', K. Heimann', S. TrepelsKottek', T. Orlikowsky

'Uniklinik der RWTH Aachen, Klinik für Kinder- und Jugendmedizin, Sektion Neonatologie, Aachen, Deutschland, ${ }^{2} U$ Uniklinik der RWTH Aachen, Fachübergreifende Einheit Notaufnahme, Aachen, Deutschland, ${ }^{3}$ Uniklinik der RWTH Aachen, Klinik für Anästhesiologie, Aachen, Deutschland

Hintergrund und Fragestellung. Präklinische Reanimationen sind im Kindes- und Jugendalter selten, aber mit einem schlechten Überleben von 5-10\% assoziiert. Der Anteil der Ereignisse, die trotz intensiver notärztlicher und intensivmedizinischer Bemühungen tragisch verlaufen, ist hoch. Dabei entstand in unserem Team der Eindruck, dass speziell Reanimationen nach nicht bezeugtem Eintritt des Kreislaufstillstands so gut wie nie einen befriedigenden Ausgang nehmen. Zur Objektivierung dieses Eindrucks haben wir retrospektiv nach Utstein-Kriterien alle präklinischen Reanimationen von Kindern und Jugendlichen untersucht, die in unserer Einrichtung von 2003 bis 2013 behandelt wurden. Vergleichbare Daten wurden in Deutschland u. W. zuletzt von Zimmermann et al. für den Raum Stuttgart publiziert.

Patienten und Methoden. Eingeschlossen wurden alle Kinder bis 18 Jahre, die vom 1.1.2003 bis zum 1.1.2014 im Uniklinikum Aachen nach präklinischer Reanimation behandelt wurden. Als Reanimation wurde jegliche Form der Herzdruckmassage oder assistierten Ventilation durch Laien oder Rettungsdienstpersonal gewertet. Die Ereignisse wurden in 3 Kategorien eingeteilt: OHCA = „out of hospital cardiac arrest“, OHRA $=$ „out of hospital respiratory arrests“ und NCOHCA $=$ „non confirmed out of hospital cardiac arrest".

Ergebnisse. Fallzahlen: Gesamt $\mathrm{n}=75$ (100\%), davon $\mathrm{n}=43 \quad(57,3 \%)$ OHCA, $\mathrm{n}=19(25,3 \%)$ OHRA, $\mathrm{n}=13 \quad(19,3 \%)$ NC OHCA. Ursachen: Trauma $n=19$ (25,3\%), Ertrinken $n=12(16 \%)$, SIDS/ALTE $n=11(14,6 \%)$, Krampfanfälle $n=8(10,7 \%)$, andere $n=25(33,4 \%)$. Ereignisort: häusliche Umgebung $\mathrm{n}=54$ (72\%), Straßenverkehr oder Freizeitaktivitäten jeweils $n=8(10,7 \%)$, Schule $n=3(4 \%)$, unbekannt $n=2(2,7 \%)$. Zeugen/Erste Hilfe durch Laien: bezeugt $n=44(58,7 \%)$, Laien-Ersthilfe $n=40 \quad(55,3 \%)$. Unter den OHCA überlebten 6 von 16 Kindern (37,2\%, n=2 unbekannt) ohne Laienreanimation. Initialer Herzrhythmus: Sinusrhythmus $\mathrm{n}=31$ $(41,2 \%)$, Asystolie $n=24(32 \%)$, Bradykardie $n=5(6,7 \%)$, PEA $n=4(5,3 \%)$, Kammerflimmern $\mathrm{n}=3$ (4\%), unbekannt $\mathrm{n}=8$ (10,6\%). Epinephrin-Boli: $\mathrm{n}=27(62,8 \%)$ der OHCA-Patienten, $\mathrm{n}=7(16,3 \%)$ mehr als drei Dosen. Transport unter CPR: $n=17$ (22,6\%), davon $n=2(2,7 \%)$ Überlebende. Outcome: $n=36$ (48\%) Entlassung nach Hause, $n=7$ (9,3\%) Verlegung in Rehabilitationsklinik, $n=34$ (42,7\%) verstorben. Von den Reanimationsfällen mit unbezeugtem Eintritt des Atem- oder Kreislaufstillstands $(\mathrm{n}=19)$ überlebten $\mathrm{n}=5(26,3 \%)$.

Diskussion. Auffällig an dieser Studie ist, dass unsere Überlebensrate in der OHCA-Kategorie mit 32,56\% mehr als 3-fach höher ist, als in der Literatur angegeben. Dies ist vorrangig darauf zurückzuführen, dass bisher in diese Auswertung nur jene Patienten eingeschlossen wurden, die primär oder sekundär in unsere Klinik transportiert wurden. Es ist geplant, den Datensatz im Sinne einer populationsbasierten Datenerhebung um die Fälle zu erweitern, die am Einsatzort verstorben sind. Für uns lehrreich war die Erkenntnis, dass auch von den unbezeugten präklinischen OHCA-Fällen 5 überlebt haben, davon 2 sogar mit einem sehr guten Outcome $(\mathrm{PCPC}=1)$. Dies entsprach ausdrücklich nicht der subjektiven Wahrnehmung der Kollegen, die diese Fälle über den Untersuchungszeitraum ärztlich betreut haben. Erstmalig in Deutschland können wir zudem für eine größere Patientengruppe Daten zum Long-Term-Survival beisteuern (Follow-up min. 1 Jahr). Bislang sind keine Patienten (zu Hause oder in einer Pflegeeinrichtung) verstorben.

\section{P11-07 \\ Komplikationen bei Knopfbatterie-Ingestionen. Zwei Fallberichte}

\section{S. Schäfer', T. Leu', J. Schiffmann'}

'Klinikum Nürnberg Süd, Klinik für Neugeborene, Kinder und Jugendliche, Nürnberg, Deutschland

Hintergrund. In den USA kommt es jährlich zu über 80.000 Fremdkörper-Ingestionen bei Kindern. Dabei handelt es sich bei ca. 1 Prozent der Fälle um Batterien. Während ungefähr die Hälfte der Fremdkörper-Ingestionen asymptomatisch verlaufen, können vor allen Knopfbatterien mit längerer Liegedauer im Ösophagus erhebliche Schäden verursachen. Bei modernen Batterien sind aufgrund der hohen Leistung vor allem der Stromfluss und die damit verbundene lokale Hydrolyse problematisch. Die häufigste Todesursache bei Kleinkindern mit BatterieIngestion ist eine massive Blutung von ösophagealen Fisteln zu großen Arterien bzw. der Aorta.

Fallvorstellung. Wir berichten über zwei Fälle von Knopfbatterie-Ingestion, bei denen jeweils durch eine Ösophagusperforation mit Gefäßläsion ein lebensbedrohliche Blutung auftrat. Bei einem zehn Monate alten Säugling bestanden seit sieben Tagen Schluckbeschwerden und Fieber. In den ambulanten Vorstellungen bei verschiedenen Ärzten 
wurde jeweils der Verdacht auf eine Pharyngitis gestellt. Die Mutter verneinte wiederholt eine mögliche Fremdkörper-Ingestion. Bei blutigem Erbrechen und Teerstühlen wurde eine Ösophago-Gastro-Duodenoskopie durchgeführt. Dabei zeigte sich unterhalb des oberen Ösophagus-Sphincters ein tiefes Ulcus durch eine 3 Volt Knopfbatterie. Die Batterie wurde komplikationslos geborgen. Nach einem symptomlosen Intervall mit Befundbesserung in den Kontroll-Endoskopien erfolgte bei erneutem Bluterbrechen mit akuter Anämie und Kreislaufinsuffizienz die rasche chirurgische Ligatur eines arrodierten Gefäßes. Der zweite Fall handelt von einem elf Monate alten Säugling mit rezidivierenden Teerstühlen seit ungefähr einer Woche. Nach massivem Bluterbrechen erfolgte die Einweisung in unsere Klinik. Hier zeigte sich in der Ösophagogastroskopie in Höhe des mittleren Ösophagus eine große ösophageale Erosion mit blindem Gefäßstumpf ohne aktive Blutung. In der CT-Untersuchung des Thorax wurde eine aneurysmatische Aussackung der Aorta descendens entsprechend einer gedeckten Perforation in Richtung Ösophagus diagnostiziert. Bei plötzlicher akuter Blutung aus dem Ösophagus mit Kreislaufinsuffizienz erfolgte die sofortige operative Versorgung. Nach Abklemmen der Aorta descendens wurde das betroffene Areal reseziert und eine End-zu-End Anastomose durchgeführt. Die Ösophagusperforation wurde übernäht. Ursächlich vermuten wir die Ingestion einer Knopfbatterie mit Hängenbleiben an der aortalen Ösophagusenge. Allerdings konnte weder radiologisch noch während der Endoskopie oder intraoperativ eine entsprechende Knopfbatterie detektiert werden.

Diskussion. In unsere Fallbeschreibungen wurden Symptome wie Schluckbeschwerden und Teerstühle, die auf eine Knopfbatterie-Ingestion hinweisen können, übersehen. Durch die längere Liegezeit der Batterie kam es jeweils zu Wandschädigungen des Ösophagus und Gefäßblutungen. Bei jedem Verdacht auf eine Fremdkörper-Ingestion bei Säuglingen und Kleinkindern sollte daher an eine Batterie gedacht werden. Wenn die gezielte Anamnese oder die typischen Symptome einer oberen gastrointestinalen Blutung den Verdacht bestätigen, sollte nach der radiologischen Bildgebung schnellstmöglich eine Ösophagogastroduodenoskopie erfolgen. Auch nach erfolgreicher Entfernung der Batterie sind wie in unserer Fallbeschreibung Spätkomplikationen nach Tagen bis Wochen möglich.

\section{P11-08 \\ Sicherheit und Wirkung eines Hydrocortison-Protokolls bei Kindern mit therapierefraktärer arterieller Hypotension nach kardiopulmonalem Bypass}

F. Neunhoeffer', M. Hofbeck', H. Renk', C. Grenz', C. Haller'², E. Heimberg', I. Gerbig', C. Schlensak², M. Kumpf'

'Eberhard-Karls-Universität Universitätsklinik für Kinderheilkunde und Jugendmedizin, Kinderkardiologie, Pulmologie und pädiatrische Intensivmedizin, Tübingen, Deutschland, ${ }^{2}$ Universitätsklinikum Tübingen, Universitätsklinik für Thorax-, Herz- und Gefäßchirurgie, Tübingen, Deutschland

Hintergrund. Es ist wenig darüber bekannt welche Patienten mit therapierefraktärer arterieller Hypotension nach kardiopulmonalem Bypass (CPB) auf ein Hydrocortison-Protokoll mit Verbesserung der Kreislauffunktion reagieren.

Material und Methode. Die Daten von Kindern, welche nach unserem Protokoll aufgrund einer arteriellen Hypotension nach CPB Hydrocortison erhielten, wurden in den Jahren 2000-2010 retrospektiv ausgewertet.

Ergebnisse. 166 von 1273 Kindern nach CPB (150<1 Jahr und 16>1 Jahr) wurden in diese Studie eingeschlossen. Das Hydrocortison-Protokoll verbesserte die Kreislauffunktion signifikant, erhöhte den Blutdruck, reduzierte den Katecholaminbedarf und das Laktat im Serum bei allen Kindern >1 Jahr und bei 82\% der Säuglinge (123 von 150). Non-Responder $<1$ Jahr waren signifikant jünger, leichter, hauptsächlich männlichen Geschlechts und hatten eine signifikant längere CPB-Dauer. Das Laktat im Serum und der Paediatric Risk Of Mortality Score (PRISM) waren signifikant höher bei Non-Respondern zum Zeitpunkt des Beginns der Hydrokortisontherapie. Die Mortalität war signifikant höher bei NonRespondern (2,44\% vs. $13,5 \%$; $\mathrm{p}=0,0008)$. Das Hydrocortison-Protokoll zeigte keinerlei Nebenwirkungen wie Elektrolytverschiebungen oder Hyperglykämien.

Schlussfolgerung. Ein Hydrocortison-Protokoll bei arterieller Hypotension nach CPB ist sicher, aber nicht alle Säuglinge zeigten eine Verbesserung der Kreislauffunktion. Ein Nicht-Ansprechen war mit einer signifikant höheren Mortalität verbunden.

\section{Posterwalk 12 - Neonatale Neurologie}

\section{P12-01}

\section{Sauerstoff schädigt die weiße Substanz des unreifen Kleinhirns ohne signifikante Aktivierung von Mikroglia}

V. Brockmöller', T. Scheuer', C. Bührer', S. Endesfelder', T. Schmitz'

'Charité - Universitätsmedizin Berlin, Klinik für Neonatologie, Berlin, Deutschland

Hintergrund. Neue Studien zeigen, dass neurologische Schädigungen nach Frühgeburt neben dem Großhirn oft auch das Kleinhirn betreffen. Das Kleinhirn sehr unreifer Frühgeborener ist bei starkem Wachstum besonders vulnerabel. Eine verminderte Volumenzunahme als Zeichen einer Kleinhirnschädigung Frühgeborener kann häufig beobachtet werden. Die Ursachen hierfür sind wenig verstanden, diskutiert werden Hypoxie/Ischämie, Infektion/Inflammation und Hyperoxie. Ziel unserer Studie war es, den Einfluss von Hyperoxie auf die Entwicklung der weißen Substanz des Kleinhirns im Rattenhirn und eine mögliche protektive Wirkung von Minozyklin zu analysieren.

Methode. Sechs Tage alte (P6) Wistar-Ratten wurden für $24 \mathrm{~h}$ bis $\mathrm{P}_{7}$ unter $80 \% \mathrm{O}_{2}$ gehalten, gefolgt von einer Erholungsphase an Raumluft bis $\mathrm{P}_{9}, \mathrm{P}_{11}, \mathrm{P}_{15}$ und $\mathrm{P}_{30}$. Im Vergleich zu Kontrolltieren untersuchten wir Apoptose, Proliferation und Reifung von oligodendrogliären Vorläuferzellen (OVZ) mittels Immunhistochemie (IHC), Western Blot und Realtime PCR. Langzeitfolgen der Sauerstofftoxizität ermittelten wir über MRT-Volumetrie und durch Quantifizierung von Myelin-basischem Protein (MBP).

Ergebnisse. Akute Schädigungen der Oligodendroglia im Kleinhirn nach Hyperoxie zeigte sich mit verminderter Proliferation von $\mathrm{NG}_{2}+\mathrm{Ki} 67+\mathrm{OVZ}$ von P7-Ratten, die auch nach 2 und 4 Tagen Erholung an Raumluft persistierte. In diesem Zeitraum fanden sich nach neonataler Hyperoxie zudem eine signifikante Reduktion reifer $(\mathrm{CC} 1+)$ Oligodendrozyten, sowie eine signifikante Zunahme der apoptotischen TUNEL+ Zellen in der weißen Substanz des Kleinhirns. Langzeitfolgen nach Hyperoxie zeigten sich in einer signifikanten Volumenabnahme der Kleinhirne juveniler $\mathrm{P}_{3}$ o Ratten. Zudem war Hyperoxie-induzierte Hypomyelinisierung mit verminderter Expression von MBP nachweisbar. Eine Zunahme der Nitrotyrosinkonzentration nach Hyperoxie war mittels Western Blot festzustellen. Der oxidative Stress nach Hyperoxie war durch Gabe von Minozyklin zu verhindern. Die Analysen der immunhistochemischen Färbung Iba1 und der Genexpressionen verschiedener pro-inflammatorischer Zytokine ergaben keinen Hinweis auf eine Mikroglia-Aktivierung. Der Einsatz von Minozyklin als Neuroprotektivum während Hyperoxie-Exposition ergab eine signifikant verbesserte Kleinhirn-Entwicklung. Die anti-oxidativen Eigenschaften des Medikaments scheinen dabei im Vordergrund zu stehen.

Fazit. Das unreife Kleinhirn zeigt eine ausgeprägte Vulnerabilität gegen hohe Sauerstoff-Konzentrationen, die sowohl in akuter Schädigung von Oligodendroglia als auch in Langzeitfolgen für die weiße Substanz und die Volumenexpansion zu sehen sind. Der experimentelle Einsatz von Minozyklin deutet auf pharmakologische Präventionsmöglichkeiten mittels antioxidativer Substanzen. 
P12-02

Hydrozephalus internus beim Frühgeborenen - neonatale Ureaplasmen-Meningitis?

K. Glaser', M. Wohlleben ${ }^{2}$, C. Speer ${ }^{2}$

'Universitäts-Kinderklinik und Poliklinik, Neonatologie, Würzburg, Deutschland, ${ }^{2}$ Universitäts-Kinderklinik und Poliklinik, Würzburg, Deutschland

Hintergrund. Ureaplasmen sind Schleimhautparasiten, die den Respirations- und Urogenitaltrakt meist asymptomatisch kolonisieren. Bei Früh- und Neugeborenen sind beide als pathogene Erreger invasiver Erkrankungen, wie neonataler Pneumonie und Sepsis, beschrieben. Ein Zusammenhang zwischen perinataler Ureaplasmen-Infektion und vorzeitiger Wehentätigkeit wird ebenso vermutet wie eine Assoziation mit der bronchopulmonalen Dysplasie beim Frühgeborenen. Die Relevanz der neonatalen Ureaplasmen-Meningitis wird demgegenüber kontrovers diskutiert.

Patient. Wir berichten über einen 9 Monate alten weiblichen Säugling mit progredientem Hydrozephalus internus unklarer Genese und Entwicklungsverzögerung, verursacht durch eine mehr als 200 Tage andauernde chronische Inflammation des zentralen Nervensystems (ZNS). Das Frühgeborene der 26+3. SSW (Geburtsgewicht $940 \mathrm{~g}$ ) war zur Anlage eines ventrikulo-peritonealen (VP-)Shunts in unsere Klinik überwiesen worden. Mit Ausnahme eines moderaten Atemnotsyndroms war ein unauffälliger postnataler Verlauf dokumentiert worden; dennoch Manifestation eines Hydrozephalus internus 4 Wochen postnatal. Neurosonographisch und kernspintomographisch gelang keine Darstellung einer intraventrikulären Hämorrhagie, in repetitiven Liquor-Untersuchungen jedoch Nachweis einer ausgeprägten Inflammation des ZNS (50-86 Zellen/ $\mu$ l, Liquor-Eiweiß 600-100o mg/dl, Liquor-Glukose nicht messbar). Trotz vermuteter bakterieller Meningitis gelang kein Erregernachweis, empirische antibiotische Therapien zeigten keinen Effekt. Bei Aufnahme in unsere Klinik unverändert Befund einer Pleozytose ( 125 Zellen/ $\mu \mathrm{l})$, einer ausgeprägten Proteinerhöhung $(565 \mathrm{mg} / \mathrm{dl})$ und einer signifikant verminderten Glukosekonzentration $(<2 \mathrm{mg} / \mathrm{dl})$ im Liquor.

Verlauf. Bei Cervixinsuffizienz mit Cerclage in der Pränatalanamnese wurde die mikrobiologische Diagnostik auf Ureaplasmen und $\mathrm{My}$ coplasma hominis initiiert. Es gelang der Nachweis von Ureaplasma parvum in Liquorkultur und -PCR, kein Nachweis sonstiger Erreger. Unter i.v.-antibiotischer Therapie mit Chloramphenicol erstmals kontinuierliche und schließlich vollständige Normalisierung der Liquorbefunde. Die Therapie wurde über 3 Wochen fortgeführt, der Säugling nach unkomplizierter VP-Shunt-Anlage nach Hause entlassen. In den nachfolgenden 6 Monaten zeigte sich eine erfreuliche Aufholentwicklung.

Diskussion. Es gibt eine relevante Anzahl an Fallberichten über ZNSInfektionen durch Ureaplasmen bei Früh- und Neugeborenen. Vor allem bei sehr unreifen Frühgeborenen könnten deren Prävalenz und Relevanz deutlich höher sein als bisher vermutet. Eine UreaplasmenInfektion könnte zu einer anhaltenden Inflammation des sehr unreifen Nervensystems mit neurologischen Folgeschäden führen.

Fazit. Insbesondere bei Frühgeborenen sollten ein Hydrozephalus internus unklarer Genese sowie eine chronische Inflammation des ZNS an eine Ureaplasmen-Meningitis denken lassen. Der Nachweis der Infektion wird nur durch eine gezielte Diagnostik gelingen.
P12-03

Occipitaler Zelluntergang nach Hypoglykämie bei einem reifen Neugeborenen

V. Lieftüchter', M. Klemme', S. Herber-Jonat', K. Förster', A. Schulze', B. Ertl-

Wagner ${ }^{2}$, A. Flemmer

${ }^{1}$ Klinikum der Universität München, München, Neonatologie der Kinderklinik am Perinatalzentrum Großhadern, München, Deutschland, ${ }^{2}$ Klinikum der Universität München Klinikum Großhadern, Institut für Klinische Radiologie, München, Deutschland

Hintergrund. Ein Substratmangel, wie Hypoxie oder Hypoglykämie, können cerebral zum Zellschaden oder gar Zelluntergang führen. Nach schwerer perinataler Asphyxie kommt es bei reifen Neugeborenen typischerweise zu einer Parenchymschädigung im Bereich der Basalganglien. Weniger bekannt ist aber, dass durch einen Mangel an Glucose, im Gehirn verursachte Schädigungsmuster, welches so bei reifen Neugeborenen vorkommt. Durch bildgebende Verfahren ist jedoch ein Unterschied der beschädigten cerebralen Areale, je nach Substratmangel, festzustellen.

Kasuistik. Wie berichten von einem reifen weiblichen Neugeborenen der 39. SSW. Die Geburt erfolgte mittels Vakuumextraktion. Postnatal fielen bis auf eine kleine VE-Marke keine weiteren Auffälligkeiten auf. Am dritten Lebenstag zeigte sich das Kind in der Geburtsklinik zunehmend schlapp und trinkfauler bei gleichzeitig bestehender Hyperbilirubinämie. Aufgrund eines Serum-Bilirubinwertes von $11 \mathrm{mg} / \mathrm{dl}$ wurde das Kind zur weiteren Therapie auf unsere neonatologische Intensivstation überwiesen. Bereits im Verlauf des Transportes kam es zu einem generalisierten tonisch klonischen Krampfereignis. Der erste gemessene Blutzucker bei Ankunft lag bei $6 \mathrm{mg} / \mathrm{dl}$. Nach Glucose-Bolus und Dauerinfusion, sistierten die klinischen Zeichen einer Hypoglykämie und die Blutzuckerwerte waren im Verlauf stets im Normbereich. Ein erweitertes Neugeborenen Screening zum Ausschluss einer Gluconeogenesestörung oder anderer Ursachen einer postnatalen Hypoglykämie oder Stoffwechselstörung war unauffällig. Das initiale aEEG zeigte ein Anfallsgeschehen. Im MRT zeigten sich parietooccipital bilaterale Diffusionsrestriktionen und eine verminderte Mark-Rindendifferenzierung. Auch im Verlauf konnte bis auf die anamnestisch berichtete Malnutrition keine Ursache für die Hypoglykämie gefunden werden.

Schlussfolgerung. In der Neonatalperiode kann eine protrahierte, nicht erkannte und zu spät therapierte Hypoglykämie zu einer irreversiblen okzipitalen Parenchymschädigung des kindlichen Gehirns führen. Warum es ausgerechnet in dieser Region bei Hypoglykämie in der Neonatalperiode zu einem lokalisierten Zelluntergang kommt, bleibt unklar.

\section{P12-04}

\section{Wirksamkeit von Levetiracetam im Kollektiv einer Neugebore-} nenintensivstation

V. Lorenz', V. Giordano', D. Anastasia', M. Feucht', A. Berger', K. KlebermassSchrehof

${ }^{1}$ Medizinische Universität Wien/Universitätsklinik für Kinder- und Jugendheilkunde, Abteilung für Neonatologie, pädiatrische Intensivmedizin und Neuropädiatrie, Wien, Österreich

Hintergrund. Cerebrale Anfälle stellen die häufigsten neurologischen Notfälle in der Neugeborenenperiode dar. Es besteht ein erhöhtes Risiko für Mortalität und spätere neurologische Beeinträchtigung sowie schwer behandelbare Epilepsien bei den Überlebenden. Eine rasche und wirksame Anfallstherapie ist deswegen notwendig. Phenobarbital gilt trotz seiner nachgewiesenen neuroapoptotischen Wirkung in Studien an Tier und Mensch und dem Risiko bleibender Schädigung als FirstLine Medikation bei neonatalen Anfällen. Levetiracetam scheint ein günstigeres Wirkungsprofil zu haben, bisher gibt es jedoch nur wenige Daten über die Anwendung bei Neugeborenen.

Material und Methoden. Wir untersuchten in einer retrospektiven Analyse alle Neugeborenen an unserer Abteilung, die zwischen 2009 und 2014 mit Levetiracetam - nach initialer Therapie mit Phenobarbital - 
behandelt wurden. Der Therapieerfolg war definiert als „Erreichen völliger Anfallsfreiheit" (AF) und $\geq 50 \%$ Reduktion der Anfallshäufigkeit (AR).

Ergebnisse. Daten von 35 Neugeborenen (21 männlich) wurden ausgewertet. Das mittlere Gestationsalter betrug $37+1$, das mittlere Geburtsgewicht $3060 \mathrm{~g}$. Levetiracetam wurde durchschnittlich am 16. Lebenstag verabreicht. Ursachen der Anfälle waren vaskuläre Ischämien bei 20 (57,1\%), zerebrale Fehlbildungen bei $9(25,7 \%)$, genetische/metabolische Erkrankungen bei $5(14,3 \%)$ und ZNS-Infektionen bei 1 (2,9\%) Patienten. Vollständige Daten waren nur von 23 Patienten verfügbar. 17 (73,9\%) reagierten in den ersten 24 Stunden nach Levetiracetamgabe (11 Patienten AF, 6 Patienten AR), 2 weitere Patienten $(8,7 \%)$ reagierten innerhalb von 30 Tagen nach Therapiebeginn (1 Patient AF, 1 Patient AR). Bei einem Patienten kam es zu Agitation unter Levetiracetam, woraufhin die Therapie beendet wurde. Es wurden keine weiteren Nebenwirkungen beobachtet.

Schlussfolgerung. Unsere Ergebnisse zeigen, dass die Therapie mit Levetiracetam bei Neugeborenen wirksam und sicher ist. Prospektive kontrollierte Studien und Langzeitevaluationen über das Entwicklungsoutcome zur besseren Beurteilung der Anwendung von Levetiracetam bei Neugeborenen sind erforderlich.

\section{P12-05 \\ Inzidenz, Risikofaktoren und Ursachen von Hirnblutungen bei stationär behandelten reifen Neugeborenen}

T. Wallborn', B. Ackermann', M. Knüpfer', F. Pulzer', C. Gebauer', A. Bläser', U. Thome

'Universitätskinderklinik, Neonatologie, Leipzig, Deutschland

Hintergrund. Hirnblutungen bei reifen Neugeborenen sind eine seltene Erkrankung. Die vorhandenen Daten zur Inzidenz, insbesondere bei Patienten ohne typische klinische Symptomatik, weisen eine starke Variabilität auf. Einige Risikofaktoren, wie niedriges Geburtsgewicht, männliches Geschlecht, niedriger 5-Minuten APGAR Score und elektive Sectio, wurden beschrieben. Es existieren jedoch keine Empfehlungen für eine rationale Screeningstrategie.

Methoden. Zwischen den Jahren 2000 und 2015 erhielten 3281 reife Neugeborene mit stationärer Aufnahme auf unsere neonatologische Station während des Aufenthaltes Ultraschalluntersuchungen des Schädels. Die vorhandenen Daten wurden retrospektiv bezüglich der Inzidenz, Ursachen und möglicher Risikofaktoren von Hirnblutungen analysiert. Ergebnisse. Zwischen 2000 und 2015 wurden 4022 reife Neugeborene auf unsere Neugeborenenstation aufgenommen. Von diesen Patienten erhielten 3281 eine Ultraschalluntersuchung des Schädels. In 55 Fällen wurde eine intraventrikuläre Hämorrhagie, in 3 Fällen eine subdurale Blutung sonographisch nachgewiesen. Der größte Teil der Blutungen wurde pränatal oder bei stationärer Aufnahme festgestellt (58,6\%). In 28 von 58 Fällen $(48,3 \%)$ konnte die Ursache nicht eindeutig geklärt werden. Asphyxie (19\%), schwere Fehlbildungen $(13,8 \%)$ sowie eine komplizierte traumatische Geburt $(6,9 \%)$ waren die häufigsten identifizierbaren Gründe. Eine Thrombozytopenie oder Gerinnungsstörung (je 1 Fall) wurden nur in Ausnahmefällen beschrieben. Bezogen auf das untersuchte Patientenkollektiv zeigten sich die folgenden relativen $\mathrm{Ri}$ siken für den jeweiligen Parameter: Perinatale Hypoxie (RR 4,9), therapeutische Hypothermie (RR 6,o), therapiepflichtiger PDA (RR 3,4), katecholaminpflichtige Kreislaufverhältnisse (RR 3,8), Pneumotho$\operatorname{rax}(\mathrm{RR} 1,8)$ und Infektionen (RR 1,1). Weitere Einflussfaktoren waren die Geburtslage (Beckenendlage: 1 Blutung/299 Geburten, regelrechte Schädellage: 52 Blutungen/3415 Geburten) und der Geburtsmodus (Spontanpartus: 20 Blutungen/2329 Geburten [RR o,38], primäre Sectio: 19 Blutungen/756 Geburten [RR 2,1], sekundäre Sectio: 7 Blutungen/587 Geburten [RR o,8] und Vakuumextraktion: 8 Blutungen/271 Geburten [RR 2,2]).

Schlussfolgerung. Hirnblutungen bei reifen Neugeborenen sind selten. Neben bekannten Risikofaktoren wie Asphyxie, schweren Fehlbildungen oder Geburtstraumata blieben die Ursachen in den meisten Fällen unklar. Primäre Gerinnungsstörungen oder eine Thrombozytopenie spielten lediglich eine marginale Rolle. Mit einer Ultraschalluntersuchung des Schädels bei stationärer Aufnahme konnten die meisten der hier untersuchten Blutungen entdeckt werden.

\section{P12-06}

\section{Neuroplastizität bei prä- oder perinatal erworbenen Hirnläsio-} nen

H. Jünger', V. Mall', S. Berweck², I. Krägeloh-Mann³, M. Staudt'², S. Burdach

${ }^{1}$ Kinderklinik der TU Krankenhaus München - Schwabing, München, Deutschland, ${ }^{2}$ Klinik für Neuropädiatrie und Neurologische Rehabilitation Vogtareuth, Vogtareuth, Deutschland, ${ }^{3}$ Eberhard-Karls-Universität Universitätsklinik für Kinderheilkunde und Jugendmedizin, Tübingen, Deutschland

Bei Patienten mit prä- und perinatal erworbenen (einseitigen) Hirnläsionen wurden neuroplastische Mechanismen kortikospinaler Reorganisation auf kortikaler und subkortikaler Ebene untersucht. Rekrutiert wurden Patienten mit unterschiedlichen Läsionsmustern (perinatal erworbene Infarkte der A. cerebri media (kortikale Läsionen) und pränatal erworbene periventrikuläre Läsionen (subkortikale Läsionen), zum Einsatz kam eine komplementäre Methodik. strukturelle/funktionelle Magnetresonanztomographie [(f)MRT] inkl. Lesion-Symptom-Mapping, transkranielle Magnetstimulation (TMS), Magnetoenzephalographie (MEG), diffusionsbasierte Traktographie (DTI). Gezeigt werden konnte, dass sich nach frühen Hirnläsionen die kortikospinale Reorganisation des motorischen und somatosensorischen Netzwerks wesentlich unterscheidet. Im Gegensatz zu einer möglichen interhemisphäriellen Reorganisation des motorischen Netzwerks (M1 und Basalganglien) mit Übernahme von Funktionen durch die nicht geschädigte Hemisphäre gilt für den primär somatosensorischen Kortex $\left(\mathrm{S}_{1}\right)$ das Prinzip einer frühen Determination ohne bedeutende inter- bzw. intrahemisphärielle Reorganisation. Das Verständnis grundsätzlicher Mechanismen sensomotorischer Reorganisation sowie motorischen Lernens bei Patienten mit frühen Hirnschädigungen wird die Entwicklung neuer, spezifischer Therapien bei kongenitaler Hemiparese ermöglichen.

\section{P12-07}

\section{Functional MRI score predicts neurodevelopmental outcome in infants with intraventricular hemorrhage}

K. Goeral', G. Kasprian', R. Fuiko', L. Schmidt', M. Weninger', A. Berger', M. Olischar', K. Klebermaß-Schrehof'

'Univ. Klinik für Kinder und Jugendheilkunde, Abt. für Neonatologie, Pädiatrische Intensivmedizin und Neuropädiatrie, Wien, Österreich, ${ }^{2}$ Univ. Klinik für Radiologie, Abt. für Neuroradiologie, Wien, Österreich

Objective. Intraventricular hemorrhage (IVH) is a significant cause of morbidity and mortality in premature infants. There is a well known correlation between IVH grade and neurodevelopmental outcome. However, to our knowledge, there are only a few studies taking into account the site of the lesion. The aim of the present study was to create a tool which could serve as a prognostic indicator with regard to the severity of brain damage and long-term neurological outcome by assessing the functional areas affected by the bleeding.

Methods. Infants with grade III IVH with and without parenchymal involvement who had MRI scans obtained during their clinical course and assessment of neurodevelopmental outcome were included into this analysis. MRI: MRI scans were analyzed by the computation of a white matter (WMS), grey matter (GMS) and a combined MRI score $(\mathrm{cS})$ which included the following functional areas and pathologies: WMS: Pyramidal tract, Corpus callosum, Radiatio optica and Crossroad ( $\max =7$ points). GMS: Gyrus precentralis and postcentralis, Hippocampus and Basal ganglia ( $\max =11$ points). $\mathrm{cS}$ was calculated by the sum of WMS and GMS. Further points were added in the presence of 
periventricular leukomalacia, hydrocephalus and for lesions in the Cerebellum ( $\max =24$ points).

Outcome. Neurodevelopmental outcome was evaluated at 1 and 2 years corrected age via Bayley Scales of Infant development (BSID) and classified into 4 severity groups.

Results. Neonates with normal MDI or mild disability show significantly lower WMS, GMS and cS compared to infants with moderate or severe mental disability at 1 year corrected age. The same is true for PDI at 1 year corrected age for WMS, GMS and cS and 2 years corrected age for WMS and cS.

Conclusion. There is currently not much evidence with regard to the relevance of topography of injury when trying to predict long-term outcome in preterm infants with IVH. The proposed score might serve as a prognostic tool with regard to the severity of brain damage and longterm neurological outcome in preterm infants with IVH.

\section{P12-08}

Gestational age related reference values in preterm infants $<\mathbf{3 0}$ weeks of gestation for the amplitude-integrated EEG using the Burdjalov score

\section{Schmidt', K. Göral', V. Giordano', E. Obwegeser', C. Czaba', M. Olischar',} M. Weninger', A. Berger', K. Klebermass-Schrehof'

'Universitätsklinik für Kinder- und Jugendheilkunde; Medizinische Universität Wien, Abteilung für Neonatologie, pädiatrische Intensivmedizin und Neuropädiatrie, Wien, Österreich

Introduction. The amplitude-integrated EEG (aEEG) is becoming more important to monitor brain activity in premature infants. Studies have shown that early aEEGs correlate with later neuro- developmental outcome. Various scoring systems and missing gestational age related reference values for the aEEGs complicate the comparison of published data. The aim of our study was to establish gestational age related reference values for the aEEGs using the Burdjalov score in preterm infants $<30$ weeks of gestation.

Methods. 114 preterm infants [gestational age (GA) $<30$ weeks of gestation] who had an aEEG within the first 14 days of life were retrospectively included into the study. The aEEGs had been analyzed using the Burdjalov score [continuity (Co), cyclicity (Cy), amplitude of lower border (LB) and bandwidth span and amplitude of lower border (B)] and correlated with the gestational age.

Results. The analysis of aEEGs showed increasing total Burdjalov scores at higher GA. The subscores [continuity (Co), cyclicity (Cy), amplitude of lower border (LB) and bandwidth span and amplitude of lower border (B)] also increased with increasing GA.

Conclusion. Gestational age related reference values for the Burdjalov Score were determined in preterm infants $<30$ weeks GA. Increasing Total Burdjalov Scores correlate with increasing GA.

\section{P12-09}

\section{Sildenafil treatment in a neonatal rodent model of hypoxic- ischemic injury}

\section{J. Engels', N. Elting', I. Bendix', J. Herz', U. Felderhoff-Müser', M. Dzietko' 'Uniklinik Essen, Klinik für Kinderheilkunde I, Essen, Deutschland}

Background. Hypoxic-ischemic injury (HI) to the developing brain occurs in 1 in 1000 live births and remains a major cause of significant morbidity and mortality. A large number of survivors have long-term sequelae including seizures and neurological deficits. However the pathophysiological mechanisms of recovery after HI are not clearly understood and preventive measures or clinical treatments are non-existent or not highly effective in the clinical setting. Sildenafil as a specific PDE5 inhibitor leads to increased levels of the second messenger cGMP and has the ability to promote neuroprotection.
Objective. In this study we investigated the effect of Sildenafil treatment on activation of histological recovery and neurogenic response after an ischemic insult to the developing brain.

Methods. 9-day-old $\mathrm{C}_{57} \mathrm{BL} / 6$-mice were subjected to sham-operation or underwent ligation of the right common carotid artery followed by hypoxia (8\%) for 60 minutes. Animals were administered either a single dose of Sildenafil (10 mg/kg, ip) or received multiple doses on 5 consecutive days starting $2 \mathrm{~h}$ after hypoxia. Animals treated with vehicle served as controls. Furthermore pups received $\mathrm{BrdU}$ ( $50 \mathrm{mg} / \mathrm{kg}$, daily) from $\mathrm{P}_{10}$ to $\mathrm{P}_{14}$ and were either perfusion-fixed at $\mathrm{P}_{14}$ for immunohistochemical analysis or brains were dissected 2 and $24 \mathrm{~h}$ after hypoxia and analyzed for cGMP by means of Elisa.

Results. Based on cresylviolet staining, single and multiple sildenafil injections revealed no differences in histological injury compared to sham animals. In addition animals treated with Sildenafil showed no increase in BrdU positive cells in the striatum. Nevertheless doublecortin X as a marker for neuronal precursor cells, was enhanced after Sildenafil therapy. Furthermore cGMP was enhanced after Sildenafil therapy.

Conclusions. Here we report that single or multiple treatment with Sildenafil after HI showed no improvement in histological brain injury or promotion of cell proliferation but enhanced neuronal precursors. Our results suggest involvement of the cGMP signaling pathway after $\mathrm{HI}$ and contribute to a better understanding of neonatal HI.

\section{P12-10}

\section{Molecular mechanism of Sildenafil treatment in a neonatal rodent model of hypoxic-ischemic injury}

N. Elting', J. Engels', I. Bendix', J. Herz', U. Felderhoff-Müser', M. Dzietko' 'Uniklinik Essen, Klinik für Kinderheilkunde I, Essen, Deutschland

Background. Hypoxic-ischemic injury (HI) to the developing brain occurs in 1 in 1000 live births and remains a major cause of significant morbidity and mortality. A large number of survivors have long-term sequelaes including seizures and neurological deficits. However the pathophysiological mechanisms of recovery after $\mathrm{HI}$ are not clearly understood and preventive measures or clinical treatments are nonexistent or not highly effective. Sildenafil as a specific phosphodiesterase-5 (PDE5) inhibitor leads to increased levels of the second messenger cGMP and has the ability to promote neuroprotection.

Objective. In this study we investigated the effect of Sildenafil treatment on activation of intracellular signaling pathways, especially the involvement of the $\mathrm{PI}_{3} / \mathrm{Akt}$ and GSK-3 $\beta$ pathway after an ischemic insult to the developing brain.

Methods. 9-days-old $\mathrm{C}_{57} \mathrm{BL} / 6$-mice were subjected either to shamoperation or underwent ligation of the right common carotid artery followed by hypoxia (8\%) for 60 minutes. Animals were administered Sildenafil (10 $\mathrm{mg} / \mathrm{kg}$, ip) or vehicle $2 \mathrm{hrs}$ after hypoxia and brains were dissected 12 or $24 \mathrm{~h}$ after Sildenafil injection. PDE5 expression was analyzed using PCR and PKG-I, p-Akt, p-GSK- $3 \beta$ and $\beta$-catenin were quantified using Western Blot analysis.

Results. Here we show that the expression of PDE5 in injured animals is decreased at 12 and $24 \mathrm{~h}$. Animals treated with Sildenafil, who underwent HI showed no further difference compared to controls. Furthermore pGSK- $3 \beta$ is increased after HI at $24 \mathrm{~h}$. Sildenafil enhanced $\mathrm{p}$-Akt in treated animals whereas there was no detectable difference in PKG-I and $\beta$-catenin levels.

Conclusions. Although PKG-I and $\beta$-catenin were not upregulated at $24 \mathrm{~h}$, our data suggest that Sildenafil activates the $\mathrm{PI}_{3} /$ Akt signaling pathway through $\mathrm{PDE}_{5}$ inhibition. 


\section{Posterwalk 13 - Narkose, Sedierung und Analgesie}

\section{P13-01 \\ Effekt eines pflegegesteuerten Analgosedierungsprotokolls auf Beatmungsdauer und kumulative Dosen von Benzodiazepinen und Opiaten bei beatmeten Säuglingen und Kindern nach chirur- gischen Eingriffen auf der Intensivstation}

F. Neunhoeffer', G. Seitz ${ }^{2}$, F. Fideler', M. Kumpf', I. Gerbig', E. Heimberg', J. Fuchs ${ }^{2}$, M. Hofbeck ${ }^{1}$

'Eberhard-Karls-Universität Universitätsklinik für Kinderheilkunde und Jugendmedizin, Kinderkardiologie, Pulmologie und pädiatrische Intensivmedizin, Tübingen, Deutschland, ${ }^{2}$ Eberhard-Karls-Universität Universitätsklinik für Kinderheilkunde und Jugendmedizin, Kinderchirurgie und Kinderurologie, Tübingen, Deutschland, ${ }^{3}$ Universitätsklinikum Tübingen, Anästhesie und Intensivmedizin, Tübingen, Deutschland

Hintergrund. Es gibt nur wenige Daten über den Nutzen von standardisierten pädiatrischen Analgosedierungsprotokollen (pASP) bei kritisch kranken Kindern postoperativ auf der Intensivstation.

Ziel. Untersuchung des Effektes eines pflegegesteuerten pASP für beatmete pädiatrische Patienten auf die Beatmungsdauer, die kumulativen Dosen von Benzodiazepinen und Opiaten und das Auftreten von Entzugssymptomatik postoperativ auf der Intensivstation. Patienten und Methode. Prospektive Beobachtungsstudie vor und nach Implementierung eines pASP auf einer interdisziplinären pädiatrischen Intensivstation mit 14 Betten (ca. 8oo Patienten/Jahr) an einem Universitätsklinikum. Eingeschlossen wurden alle Kinder (o-16 Jahre), die für mindestens 24 Stunden postoperativ auf der Intensivstation lagen. Ausschlusskriterien waren Verlegung intubiert und beatmet und Versterben. Vor Implementierung erfolgte die Analgosedierung auf ärztliche Anordnung, nach Implementierung wurde die Analgosedierung durch das Pflegepersonal anhand des pASP, basierend auf validierten Skalen (COMFORT-B, NISS und SOS) gesteuert.

Ergebnisse. Die Beobachtungsgruppe bestand aus 121 Säuglingen und aus 116 Kindern >1 Jahr, die Interventionsgruppe bestand aus 134 Säuglingen und 110 Kindern $>1$ Jahr. Die Beatmungsdauer war in der Interventionsgruppe bei Säuglingen signifikant niedriger $[3,5(0,07-38,0)$ vs. 2,3 (o,9-16,o); $\mathrm{p}=0,038]$, bei Kindern $>1$ Jahr gab es keinen signifikanten Unterschied $\left[0,9(0,05-53,7)\right.$ vs. $\left.1,0\left(0,3^{-28,0}\right) ; p=0,81\right]$. Bezüglich der Intensiv Aufenthaltsdauer fand sich kein signifikanter Unterschied. Die kumulativen Benzodiazepin-Dosen waren in allen Altersgruppen signifikant niedriger [Säuglinge: $9,0 \mathrm{mg} / \mathrm{kg}(0,0-82,0)$ vs. $5,0 \mathrm{mg} / \mathrm{kg}$ $(1,0-50) ; \mathrm{p}=0,0001$. Kinder $>1$ Jahr: $5,0 \mathrm{mg} / \mathrm{kg}(0,5-58,0)$ vs. $4,0 \mathrm{mg} / \mathrm{kg}$ $(0,0-47) ; p=0,031]$. Bei Säuglingen war die kumulative Dosis an Opiaten signifikant niedriger $[5,0 \mathrm{mg} / \mathrm{kg}(0,5-41,0)$ vs. $4,0 \mathrm{mg} / \mathrm{kg}(1,0-31,0)$; $\mathrm{p}=0,016]$, wohingegen bei Kindern $>1 \mathrm{Jahr}$ kein Unterschied festzustellen war $[2,1 \mathrm{mg} / \mathrm{kg}(0,1-67)$ vs. $2,3 \mathrm{mg} / \mathrm{kg}(0,1-70) ; \mathrm{p}=0,82]$. Das Auftreten von Entzugssymptomen war in beiden Altersgruppen signifikant niedriger (Säuglinge: $41,3 \%$ vs. $15,7 \%$; $p=0,0001$. Kinder >1 Jahr: $35,3 \%$ vs. $20,0 \%$; $\mathrm{p}=0,01$ )

Schlussfolgerung. Die Einführung eines Analgosedierungsprotokolls kann insbesondere bei Säuglingen die Beatmungsdauer und die kumulative Dosis an Benzodiazepinen reduzieren. Dies führt zu einer signifikanten Abnahme an Entzugssymptomen.

\section{P13-02}

Assoziation einer Analgosedierung mit Fentanyl von Frühgeborenen mit dem entwicklungsneurologischen Outcome im korrigierten Alter von 24 Monaten

\section{S. Niedermaier', K. Förster', A. Schulze ${ }^{2}$, F. Heinen 3 , A. Hilgendorff 2}

${ }^{1}$ Klinikum der Universität München, Dr. von Haunersches Kinderspital, Neonatologie, München, Deutschland, ${ }^{2}$ Klinikum der Universität München, München, Neonatologie der Kinderklinik am Perinatalzentrum Großhadern, München, Deutschland, ${ }^{3}$ Integriertes sozialpädiatrisches Zentrum im Dr. von Haunerschen Kinderspital, München, Deutschland

Hintergrund. Fentanyl wird zur Analgosedierung Frühgeborener häufig verwendet. Über das entwicklungsneurologische Outcome nach Fentanylgabe in der Neonatalperiode ist bisher wenig bekannt. In dieser retrospektiven Analyse sollte ein möglicher Zusammenhang zwischen Fentanylgabe in der Neonatalperiode und dem Entwicklungsstand im korrigierten Alter von 24 Monaten untersucht werden.

Methodik. $\mathrm{N}=35$ Frühgeborene, die mit einem Gestationsalter $\leq 32$ SSW und/oder einem Geburtsgewicht unter $1500 \mathrm{~g}$ im Jahr 2011 im Perinatalzentrum Großhadern geboren wurden, konnten in die Untersuchung eingeschlossen werden. Ausschlusskriterien waren schwere angeborene Fehlbildungen oder der Verdacht auf eine syndromale Erkrankung. Vergleichend erfasst wurden der klinische Verlauf (intraventrikuläre Hirnblutung, Sepsis, Bronchopulmonale Dysplasie, nekrotisierende Enterokolitis, Operation), Höhe und Dauer der Fentanylgabe sowie die Ergebnisse der entwicklungsneurologischen Nachuntersuchung mit Hilfe der Bayley Scales of Infant Development, 2nd Edition im korrigierten Alter von 24 Monaten.

Ergebnisse. 34 Kinder mit einem mittleren Gestationsalter von $27+0$ SSW (24+1 SSW bis $31+5$ SSW) und einem mittleren Geburtsgewicht von $805 \mathrm{~g}$ (410 g bis $1200 \mathrm{~g}$ ) konnten im korrigierten Alter von 23 bis 26 Monaten entwicklungsneurologisch untersucht werden. Hiervon erhielten 10 Kinder mit einem mittleren Gestationsalter von $25+4$ SSW und einem mittleren Geburtsgewicht von 758,5 g eine Fentanylsedierung in einem Zeitraum von 1 bis 9o Tagen. Die beiden Gruppen der Fentanyl behandelten Kinder und der Kinder ohne Fentanyltherapie waren bezüglich Gestationsalter und Geburtsgewicht nicht signifikant unterschiedlich. In der Fentanylgruppe hatten deutlich mehr Kinder eine intraventrikuläre Blutung $\geq^{\circ} \mathrm{I}$ (60\% in der Fentanylgruppe vs. 20\% in der Vergleichsgruppe). In der entwicklungsneurologischen Nachuntersuchung zeigten keine signifikanten Unterschiede zwischen der Gruppe mit Fentanylsedierung und der Vergleichsgruppe im Hinblick auf die motorische (Psychomotor Developmental Index, PDI $79 \pm 14$ vs. $86 \pm 9, \mathrm{p}=0,10$ ) oder die kognitive (Mental Developmental Index, MDI $83 \pm 19$ vs. $94 \pm 15, p=0,09)$ Entwicklung. Auch in der Behavioural Rating Scale zeigten sich keine signifikanten Unterschiede zwischen den beiden Gruppen (Gesamtscore 101,8 $\pm 7,6$ vs. 103,9 $\pm 11,3, \mathrm{p}=0,60$ ).

Schlussfolgerung. In dieser kleinen Kohorte von Frühgeborenen vor der 32+o SSW zeigte sich kein signifikanter Unterschied nach Analgosedierung mit Fentanyl in der Neonatalperiode im Hinblick auf das entwicklungsneurologische Outcome im Alter von 24 Monaten.

\section{P13-03}

Einfluss der Prämedikation auf die Anzahl und Dauer von Intubationsversuchen bei Frühgeborenen

M. Zemlin', S. Felgentreff', B. Wittekindt'², P. Göbert', T. Rogosch', H. Buxmann', R. Schlößer², R. Maier

'Universitätsklinikum Marburg, Klinik für Kinder- und Jugendmedizin, Marburg, Deutschland, ${ }^{2}$ Klinikum der J.W. Goethe-Universität, Klinik für Kinder- und Jugendmedizin, Neonatologie, Frankfurt am Main, Deutschland, ${ }^{3}$ Universitätsklinikum Marburg, Klinik für Kinder- und Jugendmedizin, Marburg, Deutschland

Hintergrund. Eine Intubation kann für Frühgeborene einen erheblichen Schmerzreiz darstellen, Analgetika können aber auch unerwünschte Wirkungen haben, insbesondere bei Frühgeborenen. Da keine einheit- 
lichen Empfehlungen zur Prämedikation bei Intubationen existieren, differiert das Vorgehen von Klinik zu Klinik erheblich.

Fragestellung. Unterscheiden sich Anzahl der Intubationsversuche und Gesamtdauer der Intubation zwischen zwei Perinatalzentren (PNZ) mit unterschiedlichen Prämedikationsrichtlinien?

Material und Methoden. An zwei Level 1-PNZ (PNZ1 und PNZ2) wurden prospektiv in einem Zeitraum von 24 Monaten Früh- und Reifgeborene untersucht, die endotracheal intubiert wurden. Anhand einer Videodokumentation wurden Anzahl der Intubationsversuche und Gesamtdauer vom erstmaligen Einführen des Tubus in die Nase bis Erreichen der Lage der Fixierung evaluiert. Ein Intubationsversuch begann und endete jeweils, wenn der Laryngoskopspatel die Zahnleiste überschritt. Die Videoaufnahmen wurden verblindet hinsichtlich klinischer Daten und Klinik ausgewertet.

Ergebnisse. An $\mathrm{PNZ}_{1}$ wurden 30 und an $\mathrm{PNZ}_{2} 55$ Intubationen gefilmt. Durchschnittliches Geburtsgewicht war $945 \mathrm{~g}(\mathrm{PNZ1})$ und $1090 \mathrm{~g}$ (PNZ2), Gestationsalter bei beiden PNZ im Median 27+4 SSW. Der erste Intubationsversuch wurde bei $\mathrm{PNZ}_{1}$ in $72 \%$ und in $\mathrm{PNZ}_{2}$ in $84 \%$ von Assistenzärzten unternommen. In $\mathrm{PNZ}_{2}$ wurden 7 Intubationen während der Erstversorgung - alle ohne Prämedikation - durchgeführt. Die übrigen Intubationen erfolgten auf Station. In PNZ1 erfolgten alle Intubationen mit Prämedikation. Verwendet wurden Atropin, Morphin, Midazolam und Vercuronium ( $97 \%, 100 \%, 93 \%, 53 \%$ der Intubationen). In $\mathrm{PNZ}_{2}$ wurden drei elektive Intubationen ohne Prämedikation und die übrigen mit Fentanyl, Midazolam bzw. Pancuronium (87\%, 6\%, 44\%) durchgeführt. Bei den Intubationen mit Prämedikation war an PNZ die Anzahl der Intubationsversuche 2,7 (1,25; 3), Mittelwert (Quartilen) und an $\mathrm{PNZ}_{2} 3,2(2 ; 4)(\mathrm{p}=0,2)$. Die Gesamtdauer der Intubation mit Prämedikation war an PNZ1 durchschnittlich 5,1 Minuten $(2 ; 7)$ und in $\mathrm{PNZ}_{2} 8,9$ Minuten $(2,25 ; 12,75)(\mathrm{p}<0,01)$. Ohne Prämedikation (nur PNZ2) war die Anzahl $3,3(1,25 ; 3,75)$ und die Dauer 5,9 $(2,5 ; 8,75)$ Minuten.

Diskussion. An den Zentren unterschied sich die Prämedikation erheblich und reflektierte hohe Adhärenz an die lokalen Richtlinien. Anzahl der Intubationsversuche (nicht signifikant) und Gesamtdauer (signifikant) der Intubation waren bei $\mathrm{PNZ}_{1}$ geringer als bei $\mathrm{PNZ}_{2}$ Ohne Prämedikation (nur an $\mathrm{PNZ}_{2}$ durchgeführt) dauerten elektive Intubationen kürzer als mit. Dies spiegelt vermutlich die Anweisung in $\mathrm{PNZ}_{2}$ wider, dass Prämedikation nur bei Abwehr/ Schmerzreaktion des Kindes gegeben werden sollte, so dass Wartezeiten zur Applikation entstanden.

Schlussfolgerung. An PNZ1 erhielten 6\% der Kinder zwei, 40\% drei und $54 \%$ vier Medikamente, an PNZ2 erhielten $18 \%$ kein Medikament, $35 \%$ eines, $42 \%$ zwei und $5 \%$ drei Medikamente. Höhere Medikamentenzahl war mit einer kürzeren Gesamtdauer der Intubation verbunden.

\section{P13-04}

\section{Psychoemotionales Outcome von Kindern mit angeborenen Fehlbildungen des Gastrointestinaltraktes und Anästhesie in der Neontalperiode}

N. Doberschütz' E. Frankenberg², R. Dewitz², U. Rolle ', R. Schlößer', A. Allendorf' 'Klinikum der J.W. Goethe-Univ. Zentrum der Kinderheilkunde, Schwerpunkt Neonatologie, Frankfurt am Main, Deutschland, ${ }^{2}$ Klinikum der J.W. Goethe-Univ. Zentrum der Kinderheilkunde, Schwerpunkt Neuropädiatrie, Frankfurt am Main, Deutschland, ${ }^{3}$ Universitätsklinikum der Johann-Wolfgang-Goethe-Universität Frankfurt am Main, Klinik für Kinderchirurgie, Frankfurt, Deutschland

Hintergrund. Die Überlebenschancen von Kindern mit angeborenen Fehlbildungen des Gastrointestinaltraktes sind in den letzten Jahrzehnten stark gestiegen. Das entwicklungsneurologische Outcome dieser Kinder rückte in den letzten Jahren zunehmend in den Fokus der aktuellen Forschung, während die psychoemotionale Entwicklung dieser Patienten bislang kaum Beachtung fand.

Fragestellung. Ziel unserer Studie war es, das psychoemotionale Outcome von Kindern mit angeborenen Fehlbildungen des Gastrointestinal- traktes im Alter von 24 Monaten im Vergleich zu gesunden Kindern zu untersuchen.

Material und Methoden. In dieser prospektiven Fall-Kontrollstudie untersuchten wir 40 Patienten mit relevanten Fehlbildungen des Gastrointestinaltraktes, einem Gestationsalter bei Geburt $>32$ SSW und mindestens einer chirurgischen Intervention innerhalb der Neonatalperiode, die zwischen Juni 2008 und April 2011 geboren wurden. Basierend auf Informationen über den sozioökonomischen Status der Eltern, die Anzahl der Geschwister sowie die Anzahl der im Haushalt gesprochenen Sprachen wurde jedem Patienten eine gesunde Kontrollperson gleichen Geschlechtes gegenübergestellt, wobei zudem auf eine Vergleichbarkeit von Geburtsgewicht und Gestationsalter bei Geburt geachtet wurde. Alle Studienteilnehmer wurden im korrigierten Alter von 24 Monaten mittels Bayley Scales of Infant Development II Assessment inklusive der Bayley Ratingskala zur Verhaltensbeobachtung untersucht. Mittels dieser Ratingskala werden Orientierung/Kontakt, Emotionsregulation und Motorik beurteilt, woraus ein Gesamtwert ermittelt werden kann. Ermittelt wird ein Rangwert mit einem Maximum von 99. Die Ergebnisse von Patienten- und Kontrollgruppe wurden mittels Wilcoxon-Mann-Whitney-U-Test verglichen. Um eine mögliche Korrelation dieses Outcomes mit der Exposition gegenüber Anästhesie im Rahmen der chirurgischen Eingriffe zu überprüfen, wurden die Anästhesie-Protokolle analysiert und die Ergebnisse mit den Rangwerten des psychoemotionalen Outcomes korreliert.

Ergebnisse. Zwischen der Patienten- und der gesunden Kontrollgruppe bestanden keine signifikanten Unterschiede in Geburtsgewicht $(p=0,76)$, Gestationsalter bei Geburt $(p=0,82)$, Alter bei Testung $(\mathrm{p}=\mathrm{o}, 38)$, sozioökonomischem Status $(\mathrm{p}=\mathrm{o}, 22)$, Anzahl an Geschwistern $(\mathrm{p}=0,99)$ und Anzahl der im Haushalt gesprochenen Sprachen $(\mathrm{p}=\mathrm{o}, 4 \mathrm{O})$. In Bezug auf das psychoemotionale Outcome konnten zwischen den Gruppen keine signifikanten Unterschiede in den Kategorien Orientierung/Kontakt $(\mathrm{p}=0,66)$, Emotionsregulation $(\mathrm{p}=0,29)$, Motorik $(p=0,73)$ oder dem Gesamtscore $(p=0,55)$ gefunden werden. Ebenso zeigte sich keine signifikante Korrelation dieses Outcomes und der relevanten Anästhesiedaten wie dem Alter bei der ersten Narkose oder der Gesamtanzahl und -dauer der Narkosen.

Schlussfolgerung. Die psychoemotionale Entwicklung von Kindern mit Fehlbildungen des Gastrointestinaltraktes unterscheidet sich im korrigierten Alter von 24 Monaten nicht signifikant von der gesunder Kinder. Die durchgeführten Narkosen zeigen keine signifikante Korrelation zu den Outcome-Parametern. Mögliche Störungen der psychoemotionalen Entwicklung im Verlauf der weiteren Kindheit sind bisher nicht untersucht, weshalb strukturierte Langzeit-Follow-up-Programme für diese Patientengruppe unerlässlich sind.

\section{P13-05}

Medikamenten-Rotation bei kritisch kranken Kindern mit OpiatToleranz auf der pädiatrischen Intensivstation

\section{A. Hanser', M. Hofbeck', M. Kumpf', I. Gerbig', M. Esslinger', F. Neunhoeffer}

'Universitätsklinik für Kinderheilkunde und Jugendmedizin/EberhardKarls-Universität, Kinderheilkunde II (Kardiologie, Pulmologie, Intensivmedizin), Tübingen, Deutschland

Hintergrund. Opioid-Rotation ist ein gängiges Mittel um Opiat-Toleranz in der chronischen Schmerztherapie zu verhindern. Es gibt nur wenige Berichte über Medikamenten-Rotation als Therapieoption bei Opiat-Toleranz bei kritisch kranken Kindern auf der Intensivstation. Material und Methode. Alle kritisch kranken Kinder mit Opioid-Toleranz einer interdisziplinären pädiatrischen Intensivstation im Zeitraum von 2011-2013 wurden retrospektiv erfasst. Die Kinder erhielten standardisiert für mehr als 48 Stunden kontinuierlich intravenös Ketamin anstatt einer kontinuierlichen intravenösen Opiatgabe. Es wurde der Einfluss dieser standardisierten Opiatpause auf die Analgosedierungstiefe und die Opiatdosis nach Medikamenten-Rotation untersucht.

Ergebnisse. 26 Kinder [Alter 2,5 Jahre (0,1-16,0); Gewicht 11,2 kg (3,8$62,0)]$ wurden in die Studie eingeschlossen. Die Kinder bekamen im 
16,0 Tage $(4,0-34,0)$ kontinuierlich intravenous Opiate und Benzodiazepine bis die Medikamenten-Rotation begonnen wurde. Die Dosis des Ketamins betrug 4,o mg/kg/h (1,8-6,o), die Dauer der MedikamentenRotation betrug 3,0 Tage $(2,0-6,0)$. Nach Wiederbeginn der Opiatgabe benötigten die Kinder signifikant weniger Fentanyl im Vergleich zu vor Beginn der Medikamenten-Rotation [4,15 $\mu \mathrm{g} / \mathrm{kg} / \mathrm{h}(1,2-10,0)$ vs. $2,9 \mu \mathrm{g} /$ $\mathrm{kg} / \mathrm{h}(0,8-4,9) ; \mathrm{p}=0,0000008]$. Die Dosis des Benzodiazepines wurde während der Medikamentenrotation nicht verändert. Die COMFORT-B Scores verringerten sich signifikant nach Beginn der Medikamenten-Rotation [16 (11-22) vs. 13,5 (8-18); $\mathrm{p}=0,00009]$.

Schlussfolgerung. Medikamenten-Rotation bei kritisch kranken Kindern mit Opiat-Toleranz scheint den Opiatgebrauch zu reduzieren.

\section{P13-06 \\ Prospektive Studie zur Etablierung des deutschsprachigen CAPD-Scores für die Erfassung eines Delirs bei pädiatrischen Intensivpatienten}

\section{Dill', R. von Haken', C. Traube', G. Silver', J. Meyburg'}

'Zentrum für Kinder- und Jugendmedizin, Allgemeine Pädiatrie, Heidelberg, Deutschland, ${ }^{2}$ Universitätsklinikum Heidelberg, Klinik für Anästhesiologie, Heidelberg, Deutschland, ${ }^{3}$ Weill Cornell Medical College, Pediatric Critical Care, New York, USA, ${ }^{4}$ Weill Cornell Medical College, Department of Child Psychiatry, New York, USA

Hintergrund. Mit dem Cornell Assessment of Pediatric Delirium (CAPD) steht seit kurzem ein validierter Score zur Erfassung eines Delirs bei pädiatrischen Intensivpatienten zur Verfügung. Die darin verwendeten detaillierten Tabellen zur Einschätzung von Kindern unter 2 Jahren liegen bisher nur in englischer Sprache vor. Daten zur wiederholten Anwendung des Scores im zeitlichen Verlauf fehlen.

Fragestellung. Erlaubt der ins Deutsche übertragene Score die zuverlässige Erfassung eines Delirs bei pädiatrischen Intensivpatienten, und lassen sich im zeitlichen Verlauf verschiedene Muster abgrenzen?

Material und Methoden. In enger Zusammenarbeit mit den Autoren des CAPD wurde eine autorisierte deutsche Version erstellt. Diese wurde im Rahmen einer prospektiven Studie an 74 Patienten $[3,3 \pm 4,7$ $(0-17]$ Jahre, $\mathrm{m}: \mathrm{w}=38: 36)$ evaluiert, die nach geplanten Operationen eine Nachbetreuung auf der pädiatrischen Intensivstation benötigten. Ab dem Zeitpunkt des Erwachens (RASS-Score $>-3$ ) wurden durch dieselben beiden geschulten Untersucher über 5 Tage jeweils morgens und abends ein CAPD-Score sowie die klinische Einschätzung der Pflegekraft erhoben.

Ergebnisse. Von den 654 erhobenen Scores waren 158 (24,2\%) pathologisch, insgesamt wiesen 47 Patienten (63,5\%) ein Delir auf. Etwa die Hälfte dieser Patienten $(32,4 \%)$ zeigte deutlich erhöhte Werte über mehrere Tage. Bei der anderen Hälfte $(31,1 \%)$ bestand über 24 Stunden ein leichteres Delir, welches durch genauere Analysen der einzelnen Testitems von einem reinen Sedierungsüberhang abzugrenzen war. In $84,6 \%$ der Untersuchungen stimmte die klinische Einschätzung der Pflegekraft mit dem Ergebnis des Scores überein, in 8,2\% war die Einschätzung falsch, in weiteren $7,2 \%$ konnten sich die Pflegekräfte nicht festlegen, ob ein Delir vorlag. Dabei war es einfacher, einen Patienten ohne Delir korrekt einzuschätzen, als ein Delir richtig zu erkennen $(88,2$ vs. $71,7 \%)$. Hinsichtlich der deliranten Patienten zeigte sich ein signifikanter Unterschied zwischen Intensiv- und Normalstation (73,6 vs. $63,3 \%)$ bei vergleichbarer Übereinstimmungsrate für die Kinder ohne Delir (87,4 vs. 90,4\%). Lediglich 42\% der Fälle mit leichtem Delir wurden korrekt eingeschätzt verglichen mit 79,4\% der Fälle mit schwerem Delir. Auch in der Beurteilung geistig beeinträchtigter Patienten ( $\mathrm{n}=11)$ gab es signifikante Abweichungen. Nur die Hälfte aller deliranten Patienten mit geistiger Beeinträchtigung wurde korrekt eingeschätzt verglichen mit $80,2 \%$ der neurologisch gesunden.

Schlussfolgerung. Der CAPD-Score ist nach entsprechender Schulung leicht und rasch durchführbar. Bei fast zwei Drittel aller postoperativen pädiatrischen Intensivpatienten tritt während des stationären Verlaufs ein Delir auf, wobei etwa die Hälfte der betroffenen Kinder nur in den ersten 24 Stunden leichtere Symptome entwickelt. Das Erkennen eines Delirs ist schwieriger auf Normalstationen, bei Patienten mit leichtem Delir oder vorbestehender geistiger Beeinträchtigung.

\section{P13-07}

Prämedikation vor Intubation bei Früh- und Neugeborenen eine systematische Literaturübersicht

Y. Yarkin', A. Franz', D. Bassler², C. Poets ${ }^{2}$

'Eberhard-Karls-Universität Universitätsklinik für Kinderheilkunde und Jugendmedizin, Neonatologie, Tübingen, Deutschland, ${ }^{2}$ UniversitätsSpital Zürich, Klinik für Neonatologie, Zürich, Schweiz

Hintergrund und Fragestellung. Die Intubation ist eine schmerzhafte und unangenehme Maßnahme, bei der es zu unerwünschten Reaktionen des Organismus kommen kann, die die Gesamtmorbidität und Mortalität von Frühgeborenen beeinflussen können. Zudem ist das Verletzungsrisiko bei einem sich wehrenden Kind hoch. Ziel der Prämedikation vor Intubation ist die Reduktion dieser teils schwerwiegenden Reaktionen und der assoziierten Schmerzen oder idealerweise deren Verhinderung bei möglichst günstigem Wirkungs- zu Nebenwirkungsprofil.

Methode. Zur Aktualisierung und Verbesserung unserer abteilungsinternen Leitlinie wurde eine systematische Literatursuche zur Prämedikation vor Intubation bei Früh- und Neugeborenen durchgeführt. Recherchiert wurde in Pubmed im Juli 2014. Folgende Suchstrategien wurden angewendet: ((,infant, newborn“[MeSH Terms] OR (,infant“[All Fields] AND „newborn“[All Fields]) OR „newborn infant“[All Fields] AND („humans“[MeSH Terms] AND AND „infant“[MeSH Terms]) AND ((„premedication“[MeSH Terms] OR „premedication"[All Fields]) AND (,intubation“[MeSH Terms] OR ,intubation“[All Fields])) OR („mivacurium“[Supplementary Concept] OR „mivacurium“[All Fields] OR („muscles“[MeSH Terms] OR „muscles“[All Fields] OR „muscle“[All Fields]) AND relaxants[All Fields]) OR sedation[All Fields] AND (,intubation „[MeSH Terms] OR (,intubation“[All fields]). Ergebnisse. Es ergaben sich 37 RCTs, 59 Beobachtungsstudien und 45 systematische Reviews. Nach Studium der Zusammenfassungen u. ggf. Volltexte wurden 24 RCTs, 31 Beobachtungsstudien und 34 systematische Reviews als relevant für unsere Fragestellung erachtet und gingen in die Auswertung für die Erstellung der Leitlinie ein. Die Kombinationsbehandlung aus Remifentanil (schneller Wirkungseintritt, kurze Wirkungsdauer) und dem Muskelrelaxans Mivacurium (Optimierung der Intubationsbedingungen und Reduktion des Verletzungsrisikos, dadurch evtl. Reduktion der erforderlichen Opiatdosis) erscheint sinnvoll. Schlussfolgerung. Zusammenfassend ergab sich, dass es aktuell bei Früh- und Neugeborenen unzureichende Evidenz für eine strenge Empfehlung einer bestimmten Medikamentenkombination zur Prämedikation für die Intubation gibt. Für unsere abteilungsinterne Prämedikationsleitlinie haben wir uns für die Kombination aus Remifentanil und Mivacurium entschieden.

\section{Posterwalk 14 - Seltene Erkrankungen 2}

\section{P14-01}

Pseudohypoaldosteronismus (Typ III) als Ursache einer lebensbedrohlichen Hyponatriämie eines 5 Monate alten Säuglings mit Blasenekstrophie-Epispadie-Komplex

\section{E. Salamano', O. Stangl', C. Wieg}

'Klinikum Aschaffenburg, Abteilung Neonatologie u. pädiatrische Intensivmedizin, Aschaffenburg, Deutschland

Hintergrund. Der sekundäre Pseudohypoaldosteronismus ist eine seltene, transiente Resistenz der Tubuluszellen gegen Aldosteron. Er ist assoziiert mit Fehlbildungen des Urogenitaltraktes und Infektionen des Harntraktes. Eine bedrohliche Hyponatriämie ist definiert als Serumnatrium $<130 \mathrm{mmol} / \mathrm{l}$. Eine akut auftretende Hyponatriämie führt zu 
neurologischen Symptomen wie Lethargie, Kopfschmerzen, Verwirrtheit und Krampfanfällen während eine chronische Hyponatriämie lange asymptomatisch bleibt.

Fallbericht. Fünf Monate alten Säugling mit Z. n. operativ korrigiertem Blasenekstrophie-Epispadie-Komplex. Die Vorstellung erfolgte bei ausgeprägter chronischer Gedeihstörung, Toxikose, sowie schwerster Elektrolytentgleisung mit schwerster Hyponatriämie (107 mmol/l) und metabolischer Azidose ohne Laktaterhöhung. Wegweisende laborchemische Werte waren Urin-Natrium $24 \mathrm{mmol} / \mathrm{l}$, [<20 mmol/l], FeNA $0,8 \%[<0,5 \%]$, Kreatinin 0,39 [<0,42 mg/dl], Aldosteron $13.410 \mathrm{pg} / \mathrm{ml}$ [10-160 pg/ml] und Renin $3100 \mathrm{pg} / \mathrm{ml}[5,1-38,7 \mathrm{pg} / \mathrm{ml}]$. Initial erfolgte die Therapie mit Hydrocortison ( $30 \mathrm{mg} / \mathrm{m}^{2} \mathrm{KOF}$ ) und Mineralocorticoiden $(0,3 \mathrm{mg} / \mathrm{kgKG} / \mathrm{d})$. Zudem wurde die Elektrolytimbalance durch Infusionstherapie über $72 \mathrm{~h}$ ausgeglichen. Sonographisch zeigte sich nach Rehydratation ein bisher nicht bekannter vesicouretraler Reflux. Schließlich konnte nach Ausschluss eines Defektes des Mineralkortikoidrezeptorgens $\left(\mathrm{NR}_{3} \mathrm{C}_{2}\right)$ ein sekundärer Pseudohypoaldosteronismus diagnostiziert werden. Nach Erradikationstherapie eines chronischen Harnwegsinfektes durch Serratia marcescens zeigten sich die Elektrolyte ohne Substitution ausgeglichen. Der Säugling gedieh im Folgenden regelhaft.

Diskussion. Aufgrund einer vorübergehenden Resistenz der tubulären Mineralkortikoidrezeptoren, kam es trotz stark elevierter Aldosteronspiegel zu einem renalen Salzverlustsyndrom mit erhöhter Natriurese. Der Pseudohypoaldosteronismus Typ III ist eine kaum bekannte Ursache von Gedeihstörung sowie lebensbedrohlicher Elektrolytentgleisungen (Hyponatriämie, Hyperkaliämie). bei Kindern mit Fehlbildungen des Urogenitaltraktes. Sie treten meistens auf den Boden einer Harnwegsinfektion auf. Die Literaturrecherche erbrachte nur wenige Resultate $(512$ Hits in Pubmed, 48 für den sekundären Hypoaldosteronismus). Wegweisend für die Diagnostik ist die erhöhte Natriurese bei gleichzeitig ausgeprägter Hyponatriämie. Therapeutisch ist, neben dem Ausgleich der Elektrolyte, die Sanierung des Harnwegsinfektes und der zugrundeliegenden Uropathie erforderlich.

Schlussfolgerung. Bei Kindern mit Harnwegsanomalien mit Elektrolytentgleisungen sollte an einen Pseudohypoaldosteronismus Typ III gedacht werden, der, wenn erkannt, rasch und gezielt behandelt werden kann.

\section{P14-02}

\section{Neonatale Alloimmun-Hepathopathie (neonatale Hämochroma- tose) als häufiger Grund für eine Austauschtransfusion}

\section{N. Klingenberg', T. Schmitz', M. Berns', C. Bührer' \\ ${ }^{1}$ Charite Kliniken f. Kinderheilkunde und Kinderchirugie, Neonatologie, Berlin, Deutschland}

Hintergrund. Mit Einführung der Anti-D-Prophylaxe in den sechziger Jahren haben Häufigkeit und Schweregrad des rhesusantikörperbedingten Morbus haemolyticus als Hauptursache für eine Blutaustauschtransfusion stark abgenommen.

Fragestellung. Welche Diagnosen führen heutzutage zu Austauschtransfusionen?

Material und Methoden. Retrospektive Fallanalyse, gestützt auf das Krankenhausinformationssystem.

Ergebnisse. Von den 21 in den Jahren 2009-2014 in der Neonatologie der Charité Berlin durchgeführten Austauschtransfusionen erfolgten 13 wegen eines (pränatal bekannten) Morbus haemolyticus (mittlere Schwangerschaftsdauer 34 2/7 SSW, [min $283 / 7$ bis $\max 39$ 4/7]) und acht aufgrund einer neonatalen Alloimmun-Hepathopathie (neonatale Hämochromatose; mittlere Schwangerschaftsdauer 39 o/7 SSW, [min 37 2/7 bis $\max 411 / 7])$. Die Diagnose dieser Fälle erfolgte aufgrund hoher Ferritinwerte (Median $2183 \mu \mathrm{g} / \mathrm{l}$, [min 1051 bis max. 20.394]) und hoher Transferrinsättigungen (Median 97,5\%, [min 44,3 bis max. 110,4]) bei bunter Primärsymptomatik (Icterus praecox: $n=1$, beatmungplichtiges RDS und Icterus praecox $n=1$; direkte Hyperbilirubinämie: $n=2$, Hypogklykämie mit Lakatazidose und Ketonämie: n=1, Galactosämie und Anämie: $\mathrm{n}=1$, Gewichtsverlust $>10 \%$ und direkte Hyperbilirubinämie: $\mathrm{n}=1$, Anämie, respiratorischer Insuffizienz und konnataler Infektion: $n=1$ ). Bei einer Mutter bestand eine chronisch-entzündliche Darmerkrankung mit milden Anzeichen einer primär sklerosierenden Cholangitis ohne Nachweis antimitochondrialer Antikörper. Die Austauschtransfusion erfolgte median am 11. Lebenstag (Range: 2.-48. Lebenstag), bei zwei Kindern (ausgetauscht am 22. Bzw. 48. Lebenstag) wurde später eine Lebertransplantation durchgeführt. Ein prognostischer Parameter konnte nicht identifiziert werden.

Diskussion und Schlussfolgerung. Das Voranschreiten der Erkrankung zur Leberinsuffizienz hätte möglicherweise durch eine frühere Diagnosestellung und damit eine frühere Austauschtransfusion verhindert werden können. Bei einer erneuten Schwangerschaft besteht ein hohes Wiederholungsrisiko, mit der intravenösen Gabe von Immunglobulinen an die Schwangere besteht jedoch eine wirksame Therapieoption.

\section{P14-03}

\section{Schweres Floppy-Infant-Syndrom bei Zwillingen - diagnosti-} sches Vorgehen und Stellenwert der Genetik

T. Diehl', M. Hempel', J. Denecke 3 , T. Haack', B. Grolle', D. Singer

'Universitätsklinikum Hamburg-Eppendorf, Sektion Neonatologie und Pädiatrische Intensivmedizin, Hamburg, Deutschland, ${ }^{2}$ Universitätsklinikum Hamburg-Eppendorf, Institut für Humangenetik, Hamburg, Deutschland, ${ }^{3}$ Universitätsklinikum Hamburg-Eppendorf, Klinik für Kinder- und Jugendmedizin, Hamburg, Deutschland, ${ }^{4}$ Technische Universität München, Institut für Humangenetik, Neuherberg, Deutschland, ${ }^{5}$ Altonaer Kinderkrankenhaus, Pädiatrie, Hamburg, Deutschland, ${ }^{6}$ Universitätsklinikum HamburgEppendorf, Sektion Neonatologie und Pädiatrische Intensivmedizin, Hamburg, Deutschland

Hintergrund. Ein schweres „Floppy-Infant-Syndrom“ beim Neugeborenen erfordert eine umfangreiche und zeitintensive diagnostische $\mathrm{Ab}$ klärung. Invasive Prozeduren (Muskelbiopsie) sind v.a. bei Frühgeborenen nur eingeschränkt möglich. Welche Möglichkeiten ergeben sich durch neue Methoden der genetischen Diagnostik, z. B. mittels ExomSequenzierung?

Kasuistik. Eutrophe Zwillings-Frühgeborene (dichorial-diamniot), $32+0$ SSW, männlich (1681 g/1820 g). In der Schwangerschaft Polyhydramnion und reduzierte Kindsbewegungen. Sek. Sectio bei vorzeitiger Wehentätigkeit. Intubation beider Kinder am ersten Lebenstag bei respiratorischer Erschöpfung. Endgültige Extubation erst nach mehreren Versuchen. Lange CPAP-Abhängigkeit. Ab der 1. Lebenswoche schwere muskuläre Hypotonie, reduzierte Mimik, kaum Husten, Schluckstörung.

Diagnostik. Unauffällig: CK, Sono-ZNS, MRT, Liquor, Stoffwechsel (u. a. M. Pompe, Zellweger-Syndrom, Ehlers-Danlos Typ VI). Negativer Pyridostigmin-Test. Muskelbiopsie aufgrund Unreife und geringer Muskelmasse nicht möglich. Zygotie-Bestimmung genetisch: Monozygote Zwillinge, Genetik (bei Zwilling 1): Unauffällig: Chromosomenanalyse sowie Diagnostik auf Prader-Willi-Syndrom, spinale Muskelatrophie, myotone Dystrophie. Exom-Analyse: MTM1-Genmutation (c.1505T>A); in DNA aus Lymphozyten der Mutter nicht nachweisbar. Diagnose: myotubuläre Myopathie (X-chromosomal rezessiv).

Weiterer Verlauf. Entlassung zunächst mit nächtlicher Heimbeatmung über Nasenmaske. Wiederaufnahme beider Kinder im Alter von 4,5 Monaten mit schwerer Viruspneumonie, Beatmung (u. a. HFO, iNO). Interdisziplinäre Konferenz mit den Eltern und nachfolgend Anlage Tracheostoma und PEG-Sonde. Muskelbioptisch (Immunhistochemie/ Elektronenmikroskopie) Bestätigung der myotubulären Myopathie. Heimbeatmung über Tracheostoma. Langsame kontinuierliche motorische Entwicklungsfortschritte.

Diskussion. Mittels Exom-Sequenzierung ist die Diagnosestellung einer Strukturmyopathie (z. B. der myotubulären Myopathie) beim FloppyInfant-Syndrom möglich. Ein Abwarten bis zur Durchführung einer aussagekräftigen Muskelbiopsie entfällt; ggf. kann auf diese verzichtet werden. Die frühzeitige Diagnosesicherung erlaubt ein mit den Eltern 
abgestimmtes weiteres Vorgehen unter Berücksichtigung der Gesamtprognose der Erkrankung.

\section{P14-04 \\ Glukose-Galaktose-Malabsorption - Fallbericht einer seltenen kongenitalen Diarrhoe (OMIM 606824)}

A. Große Lordemann'

'Universitätsklinikum Essen, Neonatologie, Essen, Deutschland

Fallbericht. Ein 5 Tage altes reifes Neugeborenes (40 1/7 SSW, GG 3620 g) wurde nach unkomplizierter Schwangerschaft und Geburt (Apgar 9/10/10) unter dem klinischen Bild einer Sepsis aus der Geburtsklinik überwiesen. Auffällig war eine Gewichtsabnahme von $15 \%$ seit Geburt. Anamnestisch bestanden bei dem voll gestillten Neugeborenen bis ca. 10 Stühle/d mit wässriger, spritzender Konsistenz. Laborchemisch zeigte sich eine Hypernatriämie $(\mathrm{Na} 167 \mathrm{mmol} / \mathrm{l})$ und eine metabolische Azidose ( $\mathrm{pH} 7,23$, Basenexcess - $7 \mathrm{mmol} / \mathrm{l}$, Standardbikarbonat 17,6 mmol/l) im Sinne einer hypertonen Dehydratation. Blutbild und Entzündungswerte waren unauffällig. In der Stuhlkultur wurden keine pathogenen Bakterien oder Viren nachgewiesen. Es wurde eine parenterale Ernährung mit einer Glukose-Elektrolyt-Lösung und langsame intravenöse Rehydratation begonnen und die enterale Ernährung pausiert. Hierunter kam es zu einer raschen Normalisierung der Stuhlkonsistenz und -frequenz. Nach Wiederbeginn der oralen Nahrungszufuhr mit Formalnahrung kam es jedoch erneut zu wässrigen Durchfällen, welche nach Beendigung der oralen Ernährung rasch wieder sistierten. Aufgrund des klinischen Verlaufs bestand der Verdacht auf eine Glukose-Galaktose-Malabsorbtion. Ab dem 10. Lebenstag wurde das Kind mit glukose- und galaktosefreier Spezialnahrung ernährt (Galactomin, Firma Nutrica). Hierunter blieb die Stuhlfrequenz normal. Am 15. Lebenstag wurde das Kind in gutem Allgemeinzustand entlassen. Molekulargenetisch bestätigte sich die Diagnose einer Glukose-GalaktoseMalabsorbtion durch Nachweis der Mutation c.677T>G, p, Val226Gly im SLC5A1-Gen im Homozygotenstatus. Hierbei handelt es sich um eine bislang in der Literatur nicht beschriebene Neumutation.

Schlussfolgerung. Eine Glukose-Galaktose-Malabsorbtion ist eine seltene autosomal-rezessiv vererbte Erkrankung, weltweit sind nur etwa 300 Fälle bekannt. Der Natrium-Glukose-Cotransporter, welcher durch dieses Gen kodiert wird, ist in der Bürstenmembran des Dünndarms lokalisiert und für den transmembranösen Transport von Glukose zuständig. Die Anhäufung von Glukose und Galaktose im Darm führt zu einer osmotischen Diarrhoe mit potentiell schwerer, lebensbedrohlicher Dehydratation. Charakteristisch für die malassimilatorische Diarrhö ist die geringe Menge an Elektrolyten bei hoher Osmolarität des Stuhls. Im Gegensatz zu einer sekretorischen Diarrhoe sistiert eine osmotische Diarrhoe unter Fasten. Bei neonatalen Durchfällen handelt es sich um einen Notfall. Die Glukose-Galaktose Malabsorbtion stellt eine wichtige Differentialdiagnose dar. Nahezu pathognomonisch ist das Sistieren der Durchfälle nach Pausieren der oralen Glukose-Galaktose-Zufuhr.

\section{P14-05 \\ Everolimus zur Behandlung eines riesigen kardialen Rhabdo- myoms (RM) bei einem Neugeborenen}

F. Riede', M. Weidenbach', F. Hornemann², S. Syrbe' ${ }^{2}$, R. Wagner', I. Dähnert ${ }^{1}$

'Herzzentrum Leipzig, Klinik für Kinderkardiologie, Leipzig, Deutschland, ${ }^{2}$ Klinik und Poliklinik für Kinder- und Jugendmedizin, Neuropädiatrie, Leipzig, Deutschland

Einleitung. RM sind die häufigsten Herztumore im Kindesalter und meist mit einer tuberösen Sklerose assoziiert. In der Regel zeigen sie ein gutartiges Verhalten mit einer spontanen Regredienz nach der Geburt. Sie können jedoch auch zu intrakardialen Stenosen und/oder Arrhythmien führen, die einer spezifischen Therapie bedürfen. Everolimus wird seit einigen Jahren erfolgreich in der Behandlung cerebraler Tumoren bei Kindern mit tuberöser Sklerose eingesetzt.
Kasuistik. Bei einem männlichen Feten waren multiple kardiale RM festgestellt worden, die während der Schwangerschaft ein deutliches Wachstum zeigten. Der größte Tumor füllte das Cavum des linken Ventrikels nahezu vollständig aus. Eine Tuberöse Sklerose wurde genetisch gesichert (Mutation im TSC2-Gen). Nach ausführlicher Aufklärung und Beratung der Eltern erfolgte die Geburt spontan beim Gestationsalter von 40+5 SSW (GG 2955g, GL $51 \mathrm{~cm}, \mathrm{NA}-\mathrm{pH} 7,4$ ) in einem Perinatalzentrum. Die postnatale Anpassung verlief regelrecht (Apgar 9/9/10). Nach Anlage eines Nabelvenenkatheters und Beginn einer Dauerinfusion mit Alprostadil wurde das Neugeborene im Alter von 3 Stunden in unsere Klinik verlegt. Klinisch war das Kind stabil. Auskultatorisch war ein 3/6-Systolikum nachweisbar. Obwohl dopplersonographisch nur eine milde Flussbeschleunigung von $2,8 \mathrm{~m} / \mathrm{s}$ im linksventrikulären Ausflusstrakt (LVOT) messbar war, wurde aufgrund des morphologischen Befundes mit dem Potential einer vollständigen Obstruktion des LVOT u.a. die Indikation zur chirurgischen Resektion diskutiert. Mit dem Einverständnis der Eltern wurde stattdessen am 2. Lebenstag ein Therapieversuch mit Everolimus begonnen. Die Erhaltungsdosis für den angestrebten Serum-Talspiegel von $5^{-15} \mathrm{ng} / \mathrm{ml}$ lag bei $1 \mathrm{mg} / \mathrm{m} 2 / \mathrm{d}$. Zusätzlich wurden prophylaktisch Trimethoprim-Sulfamethoxazol und Nystatin gegeben. Unter der Behandlung kam es zu einer raschen und deutlichen Größenregredienz der Tumore. Die Infusion mit Alprostadil erfolgte bis zum 3. Lebenstag; der Ductus arteriosus verschloss sich spontan. Die Behandlung mit Everolimus konnte nach 20 Tagen beendet werden. Klinisch wurden keine Nebenwirkungen beobachtet. Laborchemisch war eine leichte Hypertriglyceridämie nachweisbar. In der Durchflusszytometrie zeigte sich eine Lymphopenie mit verminderter Anzahl von B-Zellen sowie CD4- und CD8-positiven T-Zellen. Bei Entlassung am 22. Lebenstag war der Knabe in sehr gutem Allgemeinzustand. Ein Herzgeräusch war nicht mehr nachweisbar. Bei den Nachuntersuchungen über einen Zeitraum von 8 Wochen konnte ein erneutes Tumorwachstum ausgeschlossen werden.

Schlussfolgerung. Die Behandlung mit Everolimus führte bei unserem Patienten zu einer Regredienz eines riesigen RM im linken Ventrikel, die erheblich schneller verlief als sie im Rahmen des spontanen Verlaufs zu erwarten gewesen wäre. Ein komplizierter herzchirurgischer Eingriff konnte vermieden werden. Die dokumentierten immunologischen Nebenwirkungen sollten sich im Verlauf zurückbilden. Möglicherweise ist die Behandlung mit Everolimus auch für Patienten mit therapiepflichtigen kardialen RM geeignet.

\section{P14-06}

\section{Eine seltene Differentialdiagnose der pulmonalen Hypertonie:} diffuse kapilläre pulmonale Hämangiomatose

D. Stavropoulou', S. Huber-Schumacher', F. Brasch ${ }^{3}$, M. Vogel', R. Höhn ${ }^{5}$, H. Fuchs', R. Hentschel'

'Institut/Klinik: Universitätsklinikum Freiburg, Zentrum für Kinder- und Jugendmedizin, Neonatologie/Intensivmedizin, Freiburg, Deutschland, 2Universitätsklinikum Freiburg, Institut für Klinische Pathologie, Freiburg, Deutschland, ${ }^{3}$ Klinikum Bielefeld Mitte, Institut für Pathologie, Bielefeld, Deutschland, ${ }^{4}$ Pathologisches Institut/Charité, Paidopathologie \& Plazentologie, Berlin, Deutschland, ${ }^{5}$ Universitäts Herzzentrum Freiburg, Kinderherzklinik, Freiburg, Deutschland

Hintergrund. Die pulmonale Hypertonie stellt eine therapeutische Herausforderung bei Früh- und Neugeborenen dar. Ursächlich steht meist ein vorzeitiger Blasensprung mit sekundärer Lungenhypoplasie neben einer Reihe von möglichen congenitalen Erkrankungen. Die primäre diffuse pulmonale Hämangiomatose stellt eine sehr seltene und schwierig nachweisbare Ursache der pulmonalen Hypertonie dar. Eine infauste Prognose prägt die bis jetzt nur sehr wenige weltweit veröffentlichen Fälle. Fallbericht. Wir berichten über ein makrosomes Frühgeborenes der 34 . SSW mit Hydrops fetalis, der erstmalig in der 32. SSW manifest wurde. Es bestand ein Polyhydramnion. Nach einer schwierigen Primärversorgung aufgrund von Oxygenierungs- und Ventilationsstörungen mit sofortiger Intubation und beidseitiger Anlage von Pleuradrainagen 
erschwerte die persistierende pulmonale Hypertonie mit Kreislaufversagen den Krankheitsverlauf. Spezialverfahren wie HFO-Beatmung und iNO-Therapie wurden in den ersten Lebensstunden mit mäßigem Erfolg angewandt. Inhalatives Iloprost und Levosimendan wurden als Zusatztherapie angewendet. Wiederholte Surfactant-Gaben wurden zu dem Zeitpunkt ebenfalls appliziert. Im Verlauf stabilisierte sich der Zustand unter Fortsetzung der HFO- und iNO-Therapie und Hinzunahme von Sildenafil und rezidivierenden Surfactant-Gaben zunächst. Eine Reduktion der Beatmungsparameter sowie die Umstellung auf eine konventionelle Beatmungsform oder die Entfernung der Pleuradrainagen scheiterten jeweils. Im Rahmen eines pulmonalen Infekts kam es zur Entwicklung von Pneumothoraces, sowie globaler Kreislauf- und respiratorischer Insuffizienz. Das Kind verstarb am 21. Lebenstag. Die histologische Begutachtung der Lunge erbrachte eine diffuse kapilläre pulmonale Hämangiomatose $(\mathrm{PCH})$ mit pulmonaler Hypertonie und Bronchopneumonie.

Diskussion. Über eine isolierte, primäre diffuse, kapilläre pulmonale Hämangiomatose wird in der Literatur nur vereinzelt berichtet. Nach der Literatur bei Erwachsenen ist der Prozess der Erkrankung schleichend und die resultierende pulmonale Hypertonie prägt die Art der Symptomatik und der supportiven Therapie. Histologisch liegt eine unkontrollierte Kapillarproliferation in den Alveolarsepten, peribronchial, zu den benachbarten Gefäßen und im Interstitium vor. Eine gestörte Angiogenese und die Überexpression von VEGF werden ursächlich für vaskuläre Malformationen diskutiert. Keine Einigung herrscht, in wie weit reaktive Veränderungen, wie Hypoxie, ebenfalls zu einer Proliferation führen können. Eine vor kurzem erschienene Publikation legt eine Heredität nahe.

Schlussfolgerung. In Unkenntnis der Diagnose sahen wir von einer spezifischen Therapie, nach aktueller Literatur mit Imatinib oder Interferon-aza, ab. Ob diese zu einer Besserung der Symptomatik geführt hätten bleibt ungewiss. Ebenfalls ist das Langzeitoutcome solcher $\mathrm{Pa}$ tienten nicht beschrieben. Anlass zu Diskussion gibt die beschriebene Genmutation, und ob diese zukünftig bei Risikopatienten eine pränatale Diagnostik ermöglicht.

\section{P14-07}

\section{Spontane zweizeitige Zwillingsgeburt mit einem Intervall von 38 Tagen}

I. Wallot', P. Göbert', B. Arabin', J. Jansen², S. Köhler², A. Ramaswamy³, R. Maier ${ }^{1}$ Klinik für Kinder und Jugendmedizin, Marburg, Deutschland, ${ }^{2}$ Klinik für Frauenheilkunde und Geburtshilfe, Marburg, Deutschland, ${ }^{3}$ Institut für Pathologie, Marburg, Deutschland

Hintergrund. Zweizeitige Mehrlingsgeburten sind selten. Die Risiken sind mit vorzeitiger Plazentalösung oder Chorioamnionitis nicht unerheblich. In einem sehr frühen Stadium kann eine erfolgreiche Verlängerung der Schwangerschaft die Prognose für die/den intrauterin verbleibende(n) Mehrling(e) verbessern.

Fallbericht. Spontan entstandene dichoriale-diamniale Zwillingsschwangerschaft. Vorzeitiger Blasensprung des führenden Zwillings mit 22+4 SSW. Spontangeburt des ersten Zwillings (männlich) mit 26+4 SSW bei unaufhaltsamen Geburtsbestrebungen. Geburtsgewicht $720 \mathrm{~g}$. Intubation und Surfactant-Gabe im Rahmen der Erstversorgung. Extubation mit 7 Stunden, anschließend CPAP für 52 Tage. Gewicht bei Geburt des anderen Zwillings $1225 \mathrm{~g}$. Im Anschluss an die Geburt des ersten Zwillings unter Tokolyse rasche Stabilisierung der Schwangerschaft. Spontangeburt des zweiten Zwillings (weiblich) mit 32+o SSW bei Blasensprung und unaufhaltsamer Wehentätigkeit. Geburtsgewicht $1963 \mathrm{~g}$. Unkomplizierte Anpassung. Keine Atemhilfe. Keine Komplikationen. Plazentabefund: Plazenta I mit reduzierter Haftfläche, kompensatorischer Ausreifung und Endovasculopathia obliterans mit Zottenfibrose. Plazenta II mit geringer Durchblutungsstörung, fokaler Zottenfibrose und kleinem chronischen Gitterinfarkt.

Diskussion. Die meisten in der Literatur beschriebenen zweizeitigen Mehrlingsgeburten fanden zwischen 20 und 29 SSW statt und dienten dazu, die Prognose des/der intrauterin verbliebenen Kindes/r zu verbessern. In der Literatur ist zwischen den beiden Geburten ein durchschnittlicher Abstand von 19 Tagen beschrieben. Der Abstand sinkt mit fortschreitendem Gestationsalter des erstgeborenen Kindes. Er beträgt durchschnittlich 28 Tage vor Erreichen der Lebensfähigkeit, 16 Tage bei Geburt ab 25+o SSW und 8 Tage bei Geburt ab 29+o SSW. Im Vergleich dazu war in unserem Fall der erreichte Zeitgewinn mit 38 Tagen relativ hoch. Damit konnte für den zweiten Zwilling ein Gestationsalter von 32 SSW und ein Geburtsgewicht von fast $2000 \mathrm{~g}$ erreicht werden.

Schlussfolgerung. Eine zweizeitige Zwillingsgeburt auch über ein Intervall von 5 Wochen ist möglich, um Zeit für den intrauterin verbliebenen Zwilling zu gewinnen. Eine engmaschige Überwachung der Schwangerschaft in einem spezialisierten Zentrum ist in einem solchen Fall unabdingbar.

\section{P14-08}

\section{Neonatale Hämochromatose (GAL) - neue Richtlinien anhand eines Fallberichtes}

\section{A. Mittnik', H. Kurz', R. Arnhold ${ }^{2}$}

'Abteilung für Kinder- und Jugendheilkunde/Neonatologie, Donauspital, Wien, Österreich, ${ }^{2}$ Abteilung für Pathologie, Donauspital, Wien, Österreich

Einleitung. Der allgemeine Überbegriff einer neonatalen Hämochromatose (Prävalenz <1:1.000.00o) stellt lediglich die gemeinsame klinische Präsentation einer schweren fetalen Lebererkrankung dar. Währenddessen wurde die Gestational Alloimmune Liver Disease (GAL) als maternofetale Alloimmunerkrankung beschrieben, im Rahmen derer es zu einer mütterlichen IGG-Bildung gegen fetale Hepatozyten kommt, welche letztendlich durch eine Komplementaktivierung zerstört werden. Anhand eines Fallberichtes möchten wir neue Behandlungsrichtlinien, sowohl pränatal, als auch beim Neugeborenen, in Erinnerung rufen.

Fallbericht. Eltern konsanguin, St. p. intrauteriner Fruchttod unklarer Genese in 24. SSW, 2. Kind, pränatale Kontrollen wegen suspekter Lissenzephalie, unklare Anämie mit Hydrops, 3-mal Erythrozyten Transfusionen, Lungenreifung, Sectio in der $33+3$.SSW. Im Rahmen der ausführlichen Diagnostik finden sich einerseits ausgedehnte zerebrale hämorrhagische Infarzierungen beidseits, bzw. konnte bei typischer Laborkonstellation (Panzytopenie, exorbitant steigendes direktes und indirektes Bilirubin bei nur mäßig erhöhten Leberfunktionsparametern, Ferritin 17141, Transferrinsättigung $>99 \%$, Alpha-Fetoprotein 93.715 , pathologische Gerinnungswerte) der Verdacht einer GAL gestellt werden. Aufgrund der katastrophalen zerebralen Prognose erfolgt der Rückzug auf eine begleitende Palliativbetreuung, sodass der Knabe im Alter von 2 Wochen verstirbt. Postmortem kann die Diagnose einerseits durch den Nachweis einer extrahepatischen Siderose (Speicheldrüse, Pankreas, Schilddrüse etc.), andererseits intrahepatischem Komplementfaktor C5b-9 bestätigt werden. Mit der gesicherten Diagnose ergibt sich bei einer neuerlichen Schwangerschaft die Indikation für eine pränatale Therapie (i.v. IG 1 g/kg 14., 16. SSW, dann wöchentlich bis zur Entbindung), sowie, falls nötig, der Therapie bei neonataler Erkrankung (Austauschtransfusion + iv.IG $1 \mathrm{~g} / \mathrm{kg}$ ).

\section{P14-09}

\section{Transitorisch myeloproliferatives Syndrom als Ursache eines} Hydrops fetalis

\section{E. Krähling', L. Feldhahn', G. Nachtrodt', G. Froese', M. Teufel' \\ 'Klinik für Kinder- und Jugendmedizin, Böblingen, Deutschland}

Hintergrund. Ein Hydrops fetalis stellt eine schwerwiegende Notfallsituation in der Neonatologie dar. In aller Regel ist mit bedeutsamen Komplikationen schon bei der Erstversorgung zu rechnen. Dies gilt noch mehr für die Kombination aus Hydrops und extremer Frühgeburtlichkeit.

Fragestellung. Kann ein intrauterines myeloproliferatives Syndrom Ursache eines Hydrops fetalis sein? 
Material und Methoden. Wir berichten über den Verlauf eines Frühgeborenen der $26+2$ SSW mit einem intrauterin diagnostiziertem Hydrops fetalis.

Ergebnisse. Postpartal erfolgte bei respiratorischer Insuffizienz eine endotracheale Intubation sowie eine Aszitespunktion. Bei akuter Kreislaufinsuffizienz durch eine Perikardtamponade erfolgte echokardiograpisch gesteuert eine notfallmäßige Perikardpunktion. Laborchemisch imponierte eine Hyperleukozytose, Anämie, Thrombozytopenie sowie der Nachweis von Normoblasten im peripheren Blut. Bei gleichzeitig bestehender Hepatosplenomegalie, Ventrikelseptumdefekt und aufgrund entsprechender Stigmata diagnostizierter Trisomie 21 bestand der Verdacht auf ein (transientes) myeloproliferatives Syndrom. Das anhand der Immunophänotypisierung festgestellte typische Antigenexpressionsmuster bei 10\% der Blasten und der Nachweis von Mutationen des GATA-1-Transkriptionsfaktors sicherten die Diagnose. Neben rezidivierend notwendigen Gaben von Erythrozyten- und Thrombozytenkonzentraten wurden 3 Therapiezyklen mit niedrig dosiertem Cytarabin durchgeführt. Zu keinem Zeitpunkt Nachweis einer Hirnblutung. Im Verlauf zeigte sich eine Abnahme des Bedarfs an Blutprodukten sowie eine Stabilisierung der Leberinsuffizienz.

Schlussfolgerung. Ein bereits intrauterin auftretendes myeloproliferatives Syndrom kann die Ursache eines Hydrops fetalis sein. Die Diagnosestellung und deren spezifische Therapie sind die einzige Möglichkeit das Fortschreiten der Leberschädigung zu beeinflussen.

\section{P14-10}

\section{CASE Report: 12-jähriges Mädchen mit Autoimmun-Enzephalitis Typ NMDA-R-AK}

\section{Schulze', S. Montag', A. Wroblewski', C. Steen ${ }^{2}$, B. Schmidt ${ }^{3}$}

'St. Joseph Krankenhaus Berlin Tempelhof, Neonataologie und Pädiatrische Intensivmedizin, Berlin, Deutschland, ${ }^{2}$ St. Joseph Krankenhaus Berlin Tempelhof, Neuropädiatrie, Berlin, Deutschland, ${ }^{3}$ St. Joseph Krankenhaus, Berlin Tempelhof, Kinder-/Jugendmedizin, Berlin, Deutschland

Fallbericht. Ein 12-jähriges postpubertäres Mädchen wird in der Klinik aufgrund einer stattgehabten Synkope vorgestellt. Angegeben werden des Weiteren Kopfschmerzen, Schwächegefühl, Schlaflosigkeit, Palpitationen und eine unklare psychische Veränderung. Sie ist Leistungssportlerin und zeigt gute schulische Leistungen. Die Infektanamnese ist leer. Relevante Vorerkrankungen bestehen nicht. Die Familienanamnese ist ebenfalls unauffällig. Zwei Wochen zuvor erfolgte eine stationäre Abklärung eines intermittierenden AV-Blocks I. ${ }^{\circ}$, der spontan regredient war. Bei der erneuten Aufnahme zeigte sich ein AV-Block ${ }^{\circ}$ II. Klinisch präsentiert sich das Mädchen in stabilem Allgemein- und normalem Ernährungszustand mit dysthymem Affekt und klarem Sensorium. Innerhalb weniger Stunden nach Aufnahme zeigt das Mädchen psychische Beeinträchtigungen im Sinne von Phasen mit Agitation Desorientiertheit, Verwirrtheit und wechselnder Bewusstseinslage. Nach umfangreicher Diagnostik inklusive einer unauffälligen zerebralen Bildgebung wird unter dem Verdacht einer Infektion sowohl eine intravenöse antibiotische Therapie mit Cefotaxim als auch eine antivirale Therapie mit Aciclovir begonnen. Darunter zeigt sich wider Erwarten weiterhin eine rasche Zunahme der psychotischen Symptomatik mit immer längeren Intervallen zwischen den kurzen wachen, orientierten Phasen bis hin zur Notwendigkeit der Fixierung bei Eigen- und Fremdgefährdung sowie Sedierung und intensiver antipsychotischer Therapie. Am sechsten stationären Tag nach Erhalt des Liquorbefundes mit Nachweis von Auto-Antikörpern vom Typ NMDA-R-AK wurde die bisherige Therapie beendet und eine immunsuppressive Therapie mit Prednisolon und eine Immunadsorption in sieben Zyklen durchgeführt. Im Anschluss daran erfolgte eine CD2o-Depletition mit Rituximab-Gaben in vierwöchigen Abständen. Während der ersten Gabe traten vorübergehend AV-Blockierungen III. ${ }^{\circ}$ auf, die möglicherweise auf eine kardiale Mitbeteiligung der Autoantikörper schließen lassen. Drei Gaben sind bisher erfolgt und eine deutliche Regredienz der Autoantikörper im Liquor nachweisbar. Nebenbefundlich zeigen sich sowohl eine Hypovitaminose (25-OH-Vitamin D) als auch ein Folsäuremangel, ein intermittierender $\mathrm{AV}$-Block ${ }^{\circ} \mathrm{I}-{ }^{\circ} \mathrm{III}$. und eine Chromintoxikation bisher unklarer Genese. Das klinische Bild ist gekennzeichnet von ausgeprägter psychotischer Symptomatik, generalisierten tonischklonischen Krampfanfällen und Desorientiertheit.

Schlussfolgerung. Die zum Ausschluss maligner Neoplasien als Ursache dieses Autoimmunprozesses durchgeführte Diagnostik ergibt bis auf den Nachweis einer rupturierten Ovarialzyste keine Auffälligkeiten. Aktuell befindet sich die Patientin in einer neurologischen Rehabilitationseinrichtung zur Förderung der psychomentalen und statomotorischen Entwicklung. Erste Fortschritte hinsichtlich Orientiertheit und Wiedererlangung motorischer Fertigkeiten sind ersichtlich. Zurzeit benötigt die Patientin noch regelmäßig Benzodiazepine und Antipsychotika, die Prednisolongaben sind nach Reduktionsschema nahezu beendet. Die Rituximab-Gaben werden voraussichtlich bis April 2015 beendet sein.

\section{Pflege-Posterwalk}

\section{Pfl.PO-01}

\section{Primäres Bonding, auch für Früh- und kranke Neugeborene - klinische Erfahrungen}

\section{E. Vogelgesang}

'Klinikum Saarbrücken, pädiatrische Intensivstation, Schwerpunkt Neonatologie Level ${ }^{1}$, Mandelbachtal, Deutschland

Das Bonding der "normalen", gesunden Neugeborenen im Kreißsaal ist heute überall in Deutschland eine Selbstverständlichkeit. Das der kranken Neugeboren und Frühgeborene leider nicht. In der Perinatalmedizin gibt es die tradierte Vorstellung, dass Mutter und Kind in verschiedenen Disziplinen/Fakultäten behandelt werden. Dies impliziert, dass es normal sei, die Dyade aufzulösen. Die gemeinsame Versorgung von Mutter und Kind, in der Entbindungsklinik inzwischen Standard, ist auf der Neonatologie leider noch Zukunftsmusik. Zielbeformulierungen unsres Bonding-Projektes:

Wer? Mindestens 50\% aller bei uns geborenen Kinder ...

Wie? ... nach der Erstversorgung und Stabilisierung in den ersten 12 Lebensstunden zu der Mutter auf die Brust. Alternativ, wenn wegen Instabilität nicht möglich: Hautkontakt durch Handauflegen, Ansprechen, Blickkontakt Falls die Mutter nicht zur Verfügung steht, erfolgt das Bonding mit dem Vater.

Zeit? Dauer des Bondings möglichst eine Stunde, es richtet sich nach den Möglichkeiten der Mütter und Kinder.

Wo? Auf der Kinderintensivstation.

Ende? Das Ziel soll Ende April 2012 erreicht sein.

Fragen! Hat das gebondete Baby weniger Late-onset-sepsen als der Bundesdurchschnitt? Fördert das primäre Bonding die Laktation und das Stillen?

Ich stelle im Vortrag die Implementierung des primären Bondings und die Ergebnisse der beiden Fragen vor. Begleitet wird der Vortrag von vielen Bildern.

\section{Pfl.PO-02}

Die Betreuung von Neugeborenen und ihren Eltern durch Kinderärzte und Hebammen: Die Integration unterschiedlicher Sichtweisen führt zu einem ganzheitlichen Ansatz, der die Versorgung verbessern kann

T. Strahleck', A. Mora'

'Klinikum Stuttgart Olgahospital und Frauenklinik, Perinatalzentrum, Stuttgart, Deutschland

Frage: Wo und warum gibt es Differenzen in der Herangehensweise und den Schwerpunkten in der Betreuung durch Hebammen oder Kinderärzte und wie kann dies verbessert werden? Diese Frage wird anhand 
exemplarischer Überlegungen diskutiert, die Rollen werden zur besseren Verständlichkeit schematisiert überzogen dargestellt, im Bewusstsein, dass bei beiden Berufsgruppen sehr wohl auch die Aspekte der anderen Gruppe zu finden sind. Grundsätzlich haben Hebammen und Kinderärzte das gleiche Ziel: Es soll den Kindern und ihren Familien gut gehen. Hebammen betonen in der Regel die FÜR-Sorge; sie fördern die Eltern- Kind- Bindung, stärken die elterliche Feinfühligkeit und die Resilienz, vermitteln Sicherheit im Umgang mit dem Kind, bemühen sich um das Stillen, sorgen für das Wohlbefinden von Mutter und Kind: sie begleiten Familien im salutogenetischen Grundgedanken. Hebammen arbeiten eher nach dem Prinzip der Beziehungsmedizin. Kinderärzte berücksichtigen besonders die VOR-Sorge. Sie orientieren sich an der Pathogenese: sie suchen nach krankhaften oder abnormen Befunden, um Fehlentwicklungen, akute und chronische Schäden zu verhindern oder zu reduzieren, mit dem Ziel einer frühzeitigen Therapie und ggf. Förderung. Dazu gehören auch unangenehme und schmerzhafte Untersuchungen oder mehrfache Kontrollen. Da Diagnosen und prognostische Einschätzungen in einem Teil der Fälle nicht sofort verfügbar sind, entstehen für die Eltern Unsicherheiten und Ängste, die aufgefangen werden müssen. Grundlage kinderärztlichen Handels sind wissenschaftliche Erkenntnisse im Rahmen der evidenzbasierten Medizin. Eltern stellen den wichtigsten Resilienzfaktor für die günstige Entwicklung ihrer Kinder dar. Bei der Behandlung von kleinen Frühgeborenen in der Neonatologie wird dieser Tatsache durch die Einbeziehung der Eltern z. B. im Rahmen entwicklungsfördernder Pflegekonzepte Rechnung getragen. Durch die Verknüpfung von Vor- und Fürsorge, von Salutogenese und Pathogenese und von evidenzbasierter und $\mathrm{Be}$ ziehungsmedizin gewinnen wir eine ganzheitliche Sichtweise, mit der es gelingen kann, sowohl Schaden durch Krankheiten abzuwehren, wie Resilienz für eine gute Entwicklung zu fördern. Der Dialog über die unterschiedlichen Blickwinkel und den Austausch über Erfahrungen und wissenschaftliche Erkenntnis in der alltäglichen Zusammenarbeit von Kinderärzten und Hebammen lässt gegenseitiges Vertrauen und Verständnis wachsen, die Unterschiede relativieren und das gemeinsame Handeln zu einer sinnvollen Ergänzung werden. Widersprüche in der Beratung, die zusätzliche Ängste oder Unsicherheiten bei den Eltern erzeugen, werden vermieden. Die abgesprochene Verteilung von Aufgaben kann zu Arbeitsentlastung führen und die Vollständigkeit der Versorgung der Kinder und ihrer Eltern verbessern.

\section{Pfl.PO-03}

Stillbegünstigende alternative Methoden zur Zufütterung von Frühgeborenen und kranken Neugeborenen auf der NICU

\section{Braches', S. Dörl-Maschewski', J. Adler', V. Soditt'}

'Städt. Klinikum, Kinderklinik-Neonatologie, Solingen, Deutschland, ${ }^{2}$ Städtisches Klinikum Solingen $\mathrm{gGmbH}$, Zentrum für Kinder und Jugendliche - Neonatologie, Bonn, Deutschland

Hintergrund. Als dritte, von der WHO und Unicef Initiative Babyfreundlich zertifizierte Kinderklinik in Deutschland, verwenden wir auch für Frühgeborene und kranke Neugeborene ausschließlich Methoden zur Zufütterung, die das spätere Stillen und die Stillbeziehung fördern und nicht behindern.

Fragestellung. Welche Methoden fördern das Stillen und bewähren sich auf der NICU?

Material und Methoden. Wir erläutern die Verfahren und erörtern unsere klinischen Beobachtungen der verschiedenen Methoden unter den Gesichtspunkten der Praktikabilität, Effektivität und deren Anwendungsmöglichkeiten und Grenzen.

Ergebnisse. Als Ergebnis stellen wir vor, aus welchen Gründen wir welche Methoden anwenden und in der täglichen Arbeit etabliert haben.

Schlussfolgerung. Daraus ziehen wir eine Schlussfolgerung, die sicher nicht als Allgemeingültig zu verstehen ist, dennoch aber andere Kinderkliniken ermutigen soll, intensivere Stillförderung zu betreiben. Die sich bewährenden Verfahren werden empfohlen.

\section{Pfl.PO-04}

\section{Traglingskonzept im Perinatalzentrum Level 1}

\section{E. Vogelgesang'}

'Klinikum Saarbrücken, Pädiatrische Intensivstation, Schwerpunkt Neonatologie-Level ${ }^{1}$, Saarbrücken, Deutschland

Wissenschaftlicher Hintergrund. Unser Pflegekonzept auf der Kinderintensivstation richtet sich aus nach den Prinzipien der entwicklungsfördernden Pflege http://www.fruehgeborene.de/sites/default/files/ field_pblctn_file/leitsaetze.pdf und in Anlehnung an die WHO-Initiative „Kangaroo Mother Care“ http://whqlibdoc.who.int/publications/2003/9241590351_ger.pdf

Vorteile für das Kind. Das Tragen eng am Körper der Eltern hat große Vorteile für das Kind. Umso mehr für das Frühgeborene und kranke Neugeborene. Das Kind ist ein aktiver Tragling, also entsprechen wir seinen Erwartungen! Bei den frühgeborenen und kranken Kindern ist das Bonding meist unvollständig oder sogar nicht vorhanden. Das Tragen erhöht die Feinfühligkeit der Eltern und fördert das Bonding. Die FG werden viele Wochen zu früh entbunden. Anstatt im Bauch der Mutter adäquate Reize und Bewegungen in allen Ebenen zu haben, liegen sie flach und bewegungslos im Inkubator oder beim Känguruen auf der Brust. Das Tragen im Tuch verschafft ihnen die Möglichkeit dieser Erfahrungen $\rightarrow$ Entwicklungsförderung! Schaffen wir es, dass die Eltern auch zu Hause tragen, haben die Kinder „en passent“ Entwicklungsförderung auf vielen verschiedenen Ebenen, der Kopfverformung und Hypotonie des muskulären Halteapartes durch das empfohlene Rückenliegen (SIDS Prophylaxe) wird entgegen gewirkt und die Eltern gewinnen zeitlichen und räumlichen Freiraum.

Vorteile für die Eltern.

- Eltern gewinnen Sicherheit im Umgang mit dem Kind - sie werden selbstsicher.

- Eltern werden feinfühliger im Umgang und können die Zeichen des Babys besser deuten.

- Eltern schonen ihre Ressourcen - sie gewinnen zeitlichen und räumlichen Freiraum.

- Das Tuch macht Kopf, Hände und Kopf frei!

Vorteile für die Klinik.

- Konsequent eingesetzt kann das Tuch die Liegezeit verkürzen - monetärer Gewinn.

- Werbewirksam $\rightarrow$ mehr Entbindungen $\rightarrow$ monetärer Gewinn und Sicherung des Entbindungsstandortes mit angeschlossenen Level 1 Zentrum.

- Qualitätssicherungsmaßnahme - zertifizierungsrelevant!

- Imagegewinn für das Haus auf pflegerisch - fachlicher Seite $\rightarrow$ macht die MA stolz und erhöht die Motivation.

Ich beschreibe in meinem Vortrag das Traglingskonzept und den Prozess der Implementierung. Begleitet von vielen Bildern und unseren Erfahrungen. 


\section{Pfl.PO-05}

Transition to Home: ein neues Betreuungsmodell der Übergangversorgung für Familien nach einer Frühgeburt

L. Stoffel', N. Schütz Hämmerli', S. Draber', M. Nelle, K. Hirter', L. Herrmann ${ }^{6}$, D. Eichenberger zu Bonsen 7 , E. Cignacco ${ }^{8}$

'Universitätsklinik für Kinderheilkunde, Inselspital Bern, Neonatologie, Bern, Schweiz, ${ }^{2}$ Berner Fachhochschule und Inselspital Bern Universitätsklinik für Kinderheilkunde, Fachbereich Gesundheit - Neonatologie, Bern, Schweiz, ${ }^{3}$ Universitätsklinik für Kinderheilkunde, Inselspital Bern, Bern, Schweiz, ${ }^{4}$ Universitätsklinik für Kinderheilkunde, Inselspital Bern, Abteilungsleiter Neonatologie, Bern, Schweiz, ${ }^{5}$ Direktion Pflege Inselspital und Spitalnetz Bern AG, Forschung und Entwicklung, Bern, Schweiz, ${ }^{6}$ Direktion Pflege, Inselspital und Spitalnetz Bern AG, Forschung und Entwicklung, Bern, Schweiz, ${ }^{7}$ Berner Fachhochschule Fachbereich Gesundheit, Disziplin Geburtshilfe, Bern, Schweiz, ${ }^{8}$ Berner Fachhochschule Fachbereich Gesundheit, Disziplin Geburtshilfe Forschung, Bern, Schweiz

Einführung. Die Übergangsphase von der Klinik nach Hause stellt für Familien mit einem frühgeborenen Kind oder frühgeborenen Mehrlingen eine Zeit neuerlicher Verunsicherung mit physischer und emotionaler Belastung dar. Dies trifft vor allem für Familien mit sehr frühgeborenen Kindern, die in ihrer Entwicklung besonders gefährdet sind, zu. Nebst erheblichem Stress für die Eltern, kann dies zu einer verlängerten Hospitalisation des frühgeborenen Kindes führen. Zudem ist in der Schweiz die Rehospitalisationsrate bei frühgeborenen Kindern mit 35\% deutlich höher als bei termingeborenen Kindern. Verlängerte Hospitalisationen sowie Rehospitalisationen führen zu zusätzlichen Kostenbelastungen des Gesundheitssystems. In der Schweiz fehlt bislang ein pflegegeleitetes, interprofessionell abgestimmtes Angebot zur Stärkung der elterlichen Kompetenzen im Umgang mit gefährdeten frühgeborenen Kindern zu Hause. Die Idee eines Versorgungsmodells für Familien nach einer Frühgeburt, beinhaltet evidenzbasierte Interventionen zur Vorbereitung auf den Spitalaustritt des Kindes und dient der Sicherstellung einer entwicklungsfördernden Betreuung zu Hause. Dieses Vorgehen, durch gezielte Begleitung der Eltern über mehrere Monate, unterstützt das Familiensystem in der Erlangung von Stabilität. Zudem reduziert es Rehospitalisationskosten und birgt gesundheitsökonomisch ein großes Sparpotential in sich.

Ziel. Ein Advanced Practice Nurse (APN) geleitetes, interprofessionelles und nachhaltiges Modell zur Optimierung der Übergangsversorgung von Familien mit einem frühgeborenen Kind ist entwickelt und umgesetzt. Zudem sollen Wirksamkeit und Kosteneffektivität des neuen Modells überprüft werden.

Methoden. Die Entwicklung und Implementierung des neuen Versorgungsmodells basiert auf den drei folgenden Elementen: 1. Wissenschaftlicher Teil, der eine Interventionsstudie mit vorangehender Pilotstudie beinhaltet, 2.Betriebskonzept, das die Entwicklung des neuen Modelles beinhaltet und 3. Praxisentwicklung, welche die Beschreibung der Organisations- und Rollenentwicklung, sowie die direkte und konkrete Umsetzung in der Praxis beinhaltet. Das Projekt dauert von Herbst 2014 bis Dezember 2018.

Ergebnisse und Schlussfolgerung. Das Gesamtprojekt befindet sich in der Entwicklungsphase. Erste Resultate und Schlussfolgerungen der Pilotstudie werden 2017 vorgestellt.

\section{Pfl.P0-06}

\section{Premature Infant Pain Profile (PIPP) für den deutschen Sprach-} raum, erste Ergebnisse einer Pilotstudie

\section{A. Schmitt ${ }^{7}$}

\section{${ }^{1}$ Kliniken d. Stadt Saarbrücken, Kinderklinik, Saarbrücken, Deutschland}

Problem. Das Erkennen von Schmerzen bei extrem unreifen Frühgeborenen stellt für das therapeutische Team auf der neonatologischen Intensivstation eine der größten Herausforderungen unserer Zeit dar. Außer der Erfahrung der Pflegekraft und der Ärztin/des Arztes ist eine standardisierte Schmerzerfassung notwendig. Für den deutschsprachigen Raum existiert für die Gruppe der extrem unreifen Frühgeborenen kein geeignetes Schmerzerfassungsinstrument. Für den englischsprachigen Raum wurde für die genannte Zielgruppe das Premature Infant Pain Profile (PIPP) identifiziert und mit Hilfe eines im Forschungsprozess entwickelten Übersetzungs- und Äquivalenzverfahren an die deutsche Sprache und Kultur adaptiert. Mit einem ebenfalls im Forschungsprozess entwickelten Pretest-Verfahrens in Form einer Pilotstudie wurden erste Ergebnisse zum German-PIPP (G-PIPP) generiert. Fragestellung. Kann der Pretest mit der deutschen PIPP-Version zeigen, ob das Pre-Test-Design für eine geplante Validierungsstudie geeignet, die kulturelle Adaptation zwischen Original- und Zielversion gelungen und der deutschsprachige PIPP durchführbar, klinisch nützlich und reliabel ist?

Methoden. Das Forschungsdesign und die Forschungsmethode für den Pretest sind ein Methodenmix aus qualitativen und quantitativen Anteilen (die entwickelte Methode der Übersetzungsprozedur und der Äquivalenzbereiche zur Überprüfung der interkulturellen Adaptation des G-PIPP im Pretest sind qualitativ angelegt, quantitative sind u.a. die Methoden zur Beschreibung der Stichproben und von ersten Raterergebnissen).

Ergebnisse. Der Pretest zeigt, dass das Pre-Test-Studiendesign für die Validierungsstudie geeignet und die sprachliche und kulturelle Adaptation des PIPP an die deutsche Sprache und Kultur so weit gelungen ist. Es werden Aussagen zum klinischen Nutzen, Durchführbarkeit und Interrater-Reliabilität des deutschen PIPP getroffen.

Diskussion. Die Diskussion befasst sich u.a. mit der Beziehung zwischen Fremdeinschätzung und Interrater-Reliabilität, der kritischen Würdigung des PIPP und G-PIPP in Bezug auf die Durchführbarkeit und den klinischen Nutzen und der einzelnen Dimensionen der Instrumente. Des Weiteren werden Veränderungsvorschläge zur besseren Durchführbarkeit des PIPP und G-PIPP diskutiert.

Implikationen. Weitere Ergebnisse muss eine Validierungsstudie liefern.

\section{Pfl.PO-07}

Elternberatung in der neonatologischen IntensivversorgungPerspektiven von Nutzern und professionellen Akteuren im Vergleich

\section{J. Herzberg}

${ }^{1}$ Charité - Universitätsmedizin Berlin, Institut für Gesundheits- und Pflegewissenschaft, Berlin, Deutschland

Hintergrund und Zielsetzung. Im Juni 2013 sprach der Gemeinsame Bundesausschuss für die Perinatalversorgung, Level 1, eine Empfehlung für die Einführung entwicklungsadaptierter Konzepte, die sich an den individuellen Bedürfnissen des Kindes und seiner Familie orientieren und für die Etablierung institutionalisierter psychosozialer Begleitung von Eltern aus. Bereits seit 1994 halten von der pflegerischen Versorgung entbundene Gesundheits- und Kinderkrankenpflegerinnen an der Charité verschiedene edukative Unterstützungsangebote für Eltern zu früh geborener Kinder vor. Ziel dieses Forschungsvorhabens war es, subjektive Bedürfnisse der Eltern und objektiv wahrgenommene Bedarfe durch die Elternberaterinnen zu erheben und miteinander zu vergleichen. Gefragt wurde, welche Unterstützungsbedürfnisse diese Elterngruppe hat und welche (edukativen) Angebote sie sich zur Beantwortung derselben wünschen. Kontrastierend dazu wurde die Perspektive der Elternberaterinnen in die Untersuchung einbezogen.

Methoden. Zur Beantwortung wurde eine qualitative Befragung von Eltern frühgeborener Kinder $(\mathrm{n}=13)$ in verschiedenen Phasen der Krisensituation und der anbietenden Elternberaterinnen $(n=12)$ durchgeführt. Die erhobenen Daten wurden sowohl inhaltsanalytisch als auch zum Teil metaphernanalytisch ausgewertet. Anschließend wurden beide Perspektiven miteinander verglichen.

Ergebnisse. Die Ergebnisse zeigen, dass Eltern das Ereignis Frühgeburt als ein hochbelastete transitionale Phase erleben, gekennzeichnet von Angst, Orientierungslosigkeit und dem Verlust an erwünschten Inter- 
aktionskompetenzen im klinischen Umfeld. Die Bedürfnisse, die aus diesem Erleben erwachsen, unterscheiden sich zeitlich in überdauernde und phasenabhängige Bedürfnisse. Zentral sind hierbei die ausgeprägten Bedürfnisse nach kontinuierlicher Kommunikation mit und zwischen den involvierten professionellen Akteuren sowie nach umfassender Information und Aufklärung. Aus Sicht der befragten Elternberaterinnen wird das Bedürfnis der Eltern nach Kompetenz und Entlastung als bedeutsam angesehen. Über edukative Interventionen wie Information und Anleitung soll es wirkungsvoll seiner Befriedigung zugeführt werden. In der Gegenüberstellung beider dargestellter Perspektiven kann eine Diskrepanz zwischen den subjektiven Bedürfnissen der Eltern und dem professionell eingeschätzten Bedarf der professionellen Akteure in der Gesundheitsversorgung festgestellt werden.

Schlussfolgerung. In der neonatologischen Intensivversorgung wird der Blick auf krankheitsbezogene Belastungen des Kindes zunehmend um alltagsbezogene Belastungen des gesamten Systems Familie erweitert. Hierfür ist es notwendig, objektive Bedarfsbestimmungen professioneller Akteure mit Erhebungen subjektiver Unterstützungsbedürfnisse der Eltern zu ergänzen. Aus dem Vergleich dieser Perspektiven und der Identifikation von Schnittstellen kann resümiert werden, ob eine Anpassung des vorhandenen Beratungskonzeptes erfolgen muss. Hierbei gewinnt insbesondere der Blick auf die Entwicklung, Durchführung und Evaluation bedürfnisadaptierter edukativer Interventionen an Bedeutung, da sie die frühzeitige Befriedigung zentraler elterlicher Bedürfnisse fördern und somit zur eigenständigen Bewältigung der Krisensituation nach einer Frühgeburt verhelfen.

\section{Pfl.PO-08}

Eltern befähigen, ein Praxisentwicklungsprojekt der Klinik für Neonatologie am UniversitätsSpital Zürich

M. Christen', T. Karen', B. Dinten-Schmid', M. Kläusler-Troxler'

'Frauenklinik, Neonatologie, Zürich, Schweiz

Hintergrund. Die optimale Eltern-Kind-Betreuung in der Neonatologie ist uns ein großes Anliegen. Seit 1994 ist in unserer Abteilung ein interprofessionelles Elternbetreuungskonzept etabliert, welches 2006 durch das Bezugspflegesystem ergänzt wurde. Diese gemeinsame Eltern-Kind-Betreuung wird von den Eltern sehr geschätzt. Allerdings werden die erforderlichen konzeptionellen Vorgehensweisen, welche die Selbstkompetenzen, sowie die Selbstwirksamkeit der Eltern in Krisensituationen, sowie im traditionellen Familienaufbau systematisch unterstützen und fördern aktuell von Seiten des Betreuungsteams nur individuell oder sehr personenabhängig angeboten. In unserem Ansatz fehlen Eltern-Kompetenz fördernde und stärkende Ansätze.

Ziel. Implementierung des Calgary Familienpflegemodell zur Unterstützung/Stärkung der multiprofessionellen familienzentrierten Betreuung in der Neonatologie.

Methode. Das Calgary Familienassessment- und Interventionsmodell nach Wright und Leahey (2005) wird auf den Grundlagen der Aktionsforschung nach Stringer und Genat (2004), welche die drei Phasen Look, Think und Act beinhalten, implementiert. In der Look-Phase kann festgestellt werden, dass das momentane Elternbetreuungs-Konzept in der Praxis gut verankert ist. Die Eltern kennen die Betreuungspersonen, die ihrer Familie zugeteilt sind. Die verschiedenen Informationsgespräche (Anamnese-, Verlaufs- sowie Austrittsgespräch) werden gemeinsam mit einem Arzt einer Pflegefachfrau und den Eltern gemacht (Evaluation Bezugspflege 2007). Es werden den Eltern systematisch entwicklungsfördernde Konzepte wie z. B. das Kinästhetik InfantHandling angeboten. Think-Phase: Das Calgary Familienpflegemodell bietet dem Betreuungsteam Assessment- und Interventionsinstrumente an, welche den Beziehungsaufbau zu den Eltern durch gegenseitige Wertschätzung unterstützt. Das Kernelement des Modells ist die Zusammenarbeit im multiprofessionellen Team. Die Familie wird mit gezielten, ressourcenorientierten Interventionen in ihren Möglichkeiten zur eigenen Lösungsfindung bestärkt. Das Betreuungsteam bietet eine angepasste Beratung an, unterstützt die Eltern in ihrer Handlungsfä- higkeit und fördert ihre Selbsthilfefähigkeit nach der Geburt ihres zu früh geborenen oder kranken Kindes. Die Act-Phase beinhaltet diverse Projektvorbereitungen: Die Fachexpertin Stufe 4 wird gezielt auf ihre Rolle als Projektverantwortliche in der Klinik vorbereitet. Mit gezielten Teamfortbildungen sensibilisiert sie das multiprofessionelle Team für das Projekt. Dabei wird sie von der pflegerischen und medizinischen Abteilungsleitung sowie der Pflegeexpertin unterstützt. Die Projektumsetzung ist auf Sommer 2015 geplant.

Ergebnisse. Erste Ergebnisse werden Ende 2016 erwartet.

Schlussfolgerung. Die optimale Eltern-Kind-Betreuung ist eines der wichtigsten Kernelemente unserer täglichen Arbeit. Diese befindet sich im ständigen Wandel, weswegen Evaluationen bisheriger Behandlungskonzepte unabdingbar sind. 


\section{Autorenverzeichnis}

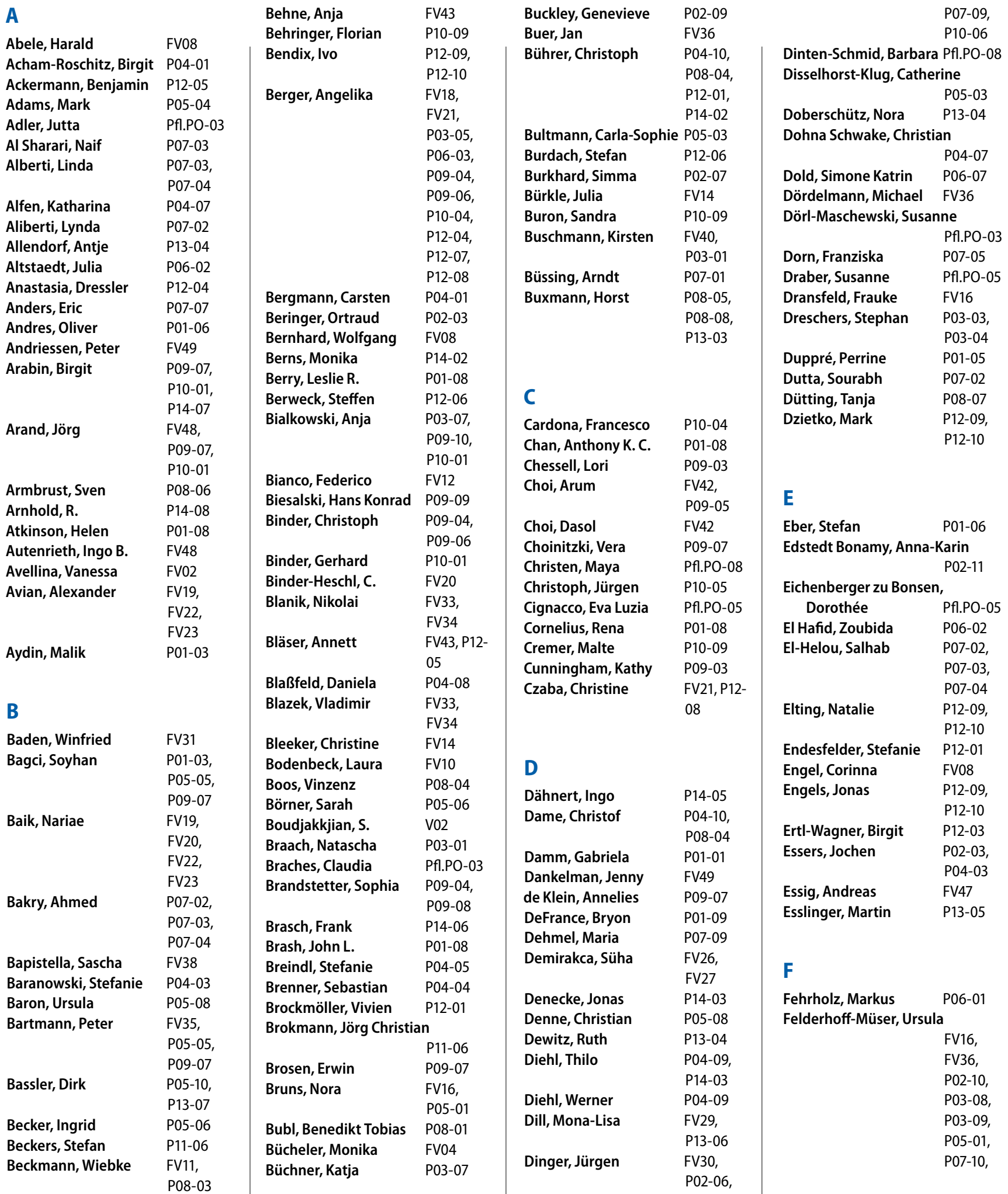




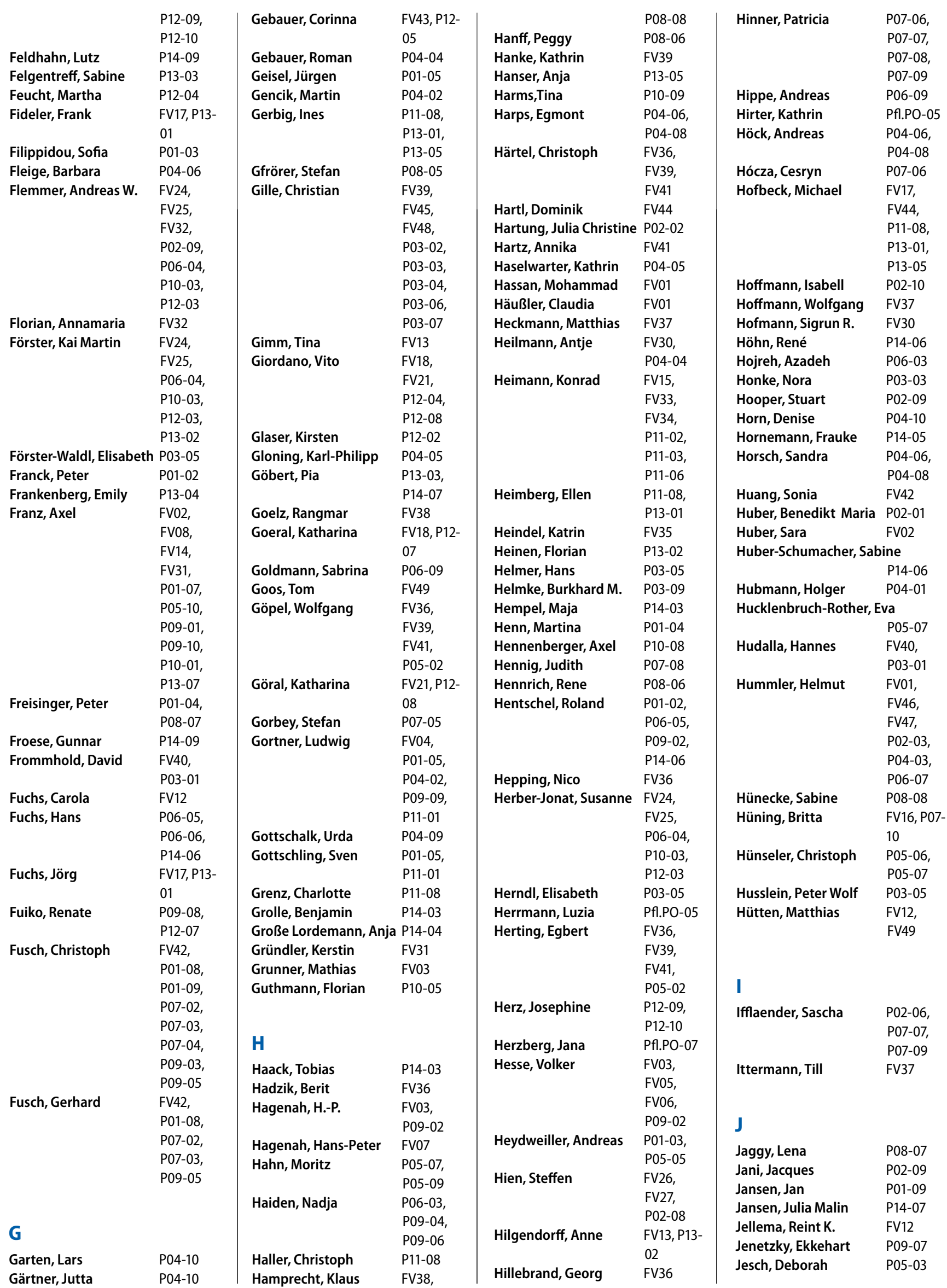




\begin{tabular}{|c|c|c|c|c|c|c|c|}
\hline $\begin{array}{l}\text { Jilma, Bernd } \\
\text { Joachim, Alexander }\end{array}$ & $\begin{array}{l}\text { P09-06 } \\
\text { P05-06 }\end{array}$ & Kreissl, Alexandra & $\begin{array}{l}\text { P09-04, } \\
\text { P09-06 }\end{array}$ & & $\begin{array}{l}\text { P13-03, } \\
\text { P14-07 }\end{array}$ & $\begin{array}{l}\text { Nachtrodt, Gerald } \\
\text { Neff, Wolfgang }\end{array}$ & $\begin{array}{l}\text { P14-09 } \\
\text { P02-08 }\end{array}$ \\
\hline John-Schuster, Gerrit & FV13 & Kribs, Angela & FV36, & Mainusch, Annika & P05-07 & Nelle, Mathias & P08-01, \\
\hline Jünger, Hendrik & P12-06 & & FV41, & Maiwald, Christian Achim & & & Pfl.PO-05 \\
\hline & & & P05-07, & & FV09 & Neuberger, Patrick & FV09 \\
\hline & & & P05-09, & Mall, Volker & P12-06 & Neuhausen, Anne & FV16 \\
\hline K & & & P08-02 & Manthei, René & FV28 & Neunhoeffer, Felix & FV17, \\
\hline Kagan, Karl & FV31 & Krieger, Tobias & P06-08 & Marschal, Matthias & FV48 & & FV44, \\
\hline Kaiser, Jan & P10-02 & Krüger, Carsten & P01-01 & Marschallek, Maria & P08-06 & & P05-10, \\
\hline Kamgari, Nona & FV13 & Kuch, Matthias & P10-07 & Marschitz, Ingrid & P11-05 & & P11-08, \\
\hline Karen, Tanja & Pfl.PO-08 & Kühne, Benjamin & P08-02 & Masjosthusmann, Katja & P08-03 & & P13-01, \\
\hline Karpf, Eva & P04-01 & Kumpf, Matthias & FV17, & Matharoo, Harpreet & P01-08 & & $\mathrm{P} 13-05$ \\
\hline Kasprian, G. & P12-07 & & FV44, & Mathes, Michaela & FV14 & Ngmus, Joan & FV24 \\
\hline Katzer, David & P01-03, & & P11-08, & Maurer, Ellen & P09-09 & Niedermaier, Sophie & $\mathrm{P} 13-02$ \\
\hline & P05-05, & & P13-01, & Mayer, Benjamin & FV01 & Niemarkt, Hendrik & FV12, \\
\hline & P09-07 & & $\begin{array}{l}P 13-05 \\
P 04-09\end{array}$ & $\begin{array}{l}\text { Mayer, Stefan } \\
\text { Mehler Katrin }\end{array}$ & $\begin{array}{l}P 05-10 \\
P 02\end{array}$ & Nikiforou Maria & $\begin{array}{l}\mathrm{FV} 49 \\
\mathrm{FV} 12\end{array}$ \\
\hline $\begin{array}{l}\text { Keppler, Oliver T. } \\
\text { Kerth, Janna-Lina }\end{array}$ & $\begin{array}{l}\text { P08-05 } \\
\text { P03-04 }\end{array}$ & Kunzmann, steпен & $\begin{array}{l}\text { P04-09, } \\
\text { P06-01 }\end{array}$ & Ivienier, Katrın & P05-06, & INIKIIOrou, IVlarıa & $\begin{array}{l}\text { FVIL, } \\
\text { FV49 }\end{array}$ \\
\hline Kieren, Tamara & FV04 & Kurz, H. & P14-08 & & P05-09 & Nitzsche, Katharina & P10-06 \\
\hline Kilbertus, H. & P02-05 & Kuss, Navina & P03-01 & Mendler, Marc Robin & FV01, & Nohr, Donatus & P09-09 \\
\hline Kindler, Annemarie & FV30 & Küster, Helmut & P02-07 & & P06-07 & Norman, Mikail & P02-11 \\
\hline Kipfmueller, Florian & FV35 & Kutscha, Christoph & P10-08 & Mensinga, Daniel & P05-05 & Nowak, Mareike & P05-09 \\
\hline Kirchner, Lieselotte & P09-08 & Kuypers, Elke & FV12, & Metzelder, Martin & P06-03 & & \\
\hline Kirste, Ariane & FV45, & & FV49 & Meyburg, Jochen & FV29, & & \\
\hline & FV48 & Kwan, Celia & FV42 & & P13-06 & 0 & \\
\hline Kislat, Andreas & P06-09 & & & Meyer, Carrie-Lynn & P07-02, & Oak, Prajakta & FV13 \\
\hline Kitchen, Marcus J. & P02-09 & & & & P07-03, & Oberthuer, André & P05-09 \\
\hline Klaassen, Ilka & P04-09 & $\mathbf{L}$ & & & P07-04 & Obwegeser, Eva & FV21, P12- \\
\hline Klambauer, Nora & P09-08 & Lachmann, Robert & P04-04 & Meyer, Sascha & P01-05, & & 08 \\
\hline Kläusler-Troxler, Marian & & Lange, Anja & FV37 & & P04-02, & Oehl-Jaschkowitz, Barba & ara \\
\hline & Pfl.PO-08 & Laux, Reinhard & FV36, & & P09-09, & & P04-02 \\
\hline Klebermaß-Schrehof, Ke & atrin & & P10-08 & & P11-01 & Oh, Jun & P04-09 \\
\hline & FV18, & Lee, Katie & P02-09 & Mileder, Lukas & FV19, & Ohl, Kim & P03-03 \\
\hline & FV21, & Lee, Sau-Young & P01-09 & & P02-04 & Ohlenbusch, Andreas & P04-10 \\
\hline & P06-03, & Leiber, Anja & P03-06, & Misselwitz, Bernd & P02-11 & Olbertz, Dirk Manfred & FV05, \\
\hline & P10-04, & & P03-07 & Mittnik, Axel & P14-08 & & FV06, \\
\hline & P12-04, & Leibold, Simone & P07-10 & Moewes, Anja & $\mathrm{P} 02-03$ & & FV07, \\
\hline & P12-07, & Lemmer, Björn & P07-05 & Mögel, Michael & P02-06, & & FV36, \\
\hline & P12-08 & Leonhardt, Steffen & FV34 & & P10-06 & & P09-02 \\
\hline Klemme, Mathias & FV25, & Leu, Thorsten & P11-07 & Mohamed, Saber & P07-03 & Olbina, Gordana & P01-07 \\
\hline & P10-03, & Lieftüchter, Victoria & P06-04, & $\begin{array}{l}\text { Mohns, Thilo } \\
\text { Mohr, Franziska }\end{array}$ & $\begin{array}{l}\text { FV49 } \\
\text { P04-10 }\end{array}$ & Olischar, Monika & FV18, \\
\hline & P12-03 & & P12-03 & Mollenhauer Jan & $\begin{array}{l}\text { P04-10 } \\
P 03-08\end{array}$ & & FV21, \\
\hline Klepper, Jörg & P10-02 & Liese, Jan & FV45, & Mollenhauer, Jan & P03-08, & & P10-04, \\
\hline Klingenberg. Norman & P14-02 & & FV48 & & P03-09 & & P12-07, \\
\hline Klotz, Daniel & P06-05, & Lindner, Wolfgang & FV46, & Montag, Sara & P14-10 & & P12-08 \\
\hline & P06-06 & & FV47 & Monz, Dominik & P09-09 & Olthoff, Julia & FV37 \\
\hline Knie, A. & FV05, & Lindtner, Claudia & P06-03 & Mora, Andrea & Pfl.PO-02 & Omran, Heymut & FV10, \\
\hline & FV06 & Lippmann, Norman & FV43 & Moser, Katja & P10-02 & & FV11, \\
\hline Knüpfer, Matthias & FV43, P12- & Loges, Niki & FV10 & Mueller, Andreas & P01-03, & & P08-03 \\
\hline & 05 & Lorenz, Laila & P01-07 & & P09-07 & Ophelders, Daan & FV12, \\
\hline Koch, Arite & P10-06 & Lorenz, Viktoria & P12-04 & Müller, Alisa & FV17 & & FV49 \\
\hline Koch. Lutz & P10-08 & Lüninghöner, Kerstin & FV24 & Müller, Andreas & FV35, & Orlikowsky, Thorsten W. & FV15, \\
\hline Koch, Sandra & P02-03 & Lüsebrink, Natalia & P08-05 & & FV41, & & FV33, \\
\hline Koch, Vera & FV08 & & & & P05-05 & & FV34, \\
\hline Koehne, Petra & P08-04 & & & Müller, Hanna & FV16, & & FV39, \\
\hline Köhler, Siegmund & P14-07 & $\mathbf{M}$ & & & P03-08, & & FV49, \\
\hline Köninger, Angela & P05-01 & Maas, Christoph & FV08, & & P03-09, & & P03-03, \\
\hline Konstantelos, Dimitrios & $\begin{array}{l}\text { P02-06, } \\
\text { P02-07 }\end{array}$ & & FV14, & Müller, Katharina & $\begin{array}{l}\text { P05-01 } \\
\text { P01-07 }\end{array}$ & & $\begin{array}{l}\text { P03-04, } \\
\text { P03-06, }\end{array}$ \\
\hline Köstlin, Natascha & P03-02, & & $\begin{array}{l}\text { b09-10, } \\
\text { rog-0, }\end{array}$ & Müller, Matthias Johan & P06-09 & & P05-03, \\
\hline & P03-06 & & P13-07 & Müller-Felber, Wolfg & P06-04 & & P11-02, \\
\hline Krägeloh-Mann, Ingebo & & Mache, Christoph Joh & ines & Mund, Iris & FV16 & & P11-03, \\
\hline & P12-06 & & P04-01, & Murthy, Prashanth & P09-03 & & P11-06 \\
\hline Krähling, Eva & P14-09 & & P11-05 & & & Oster, Isabel & P11-01 \\
\hline Kramer, Boris W. & FV12, & Maier, Rolf Felix & P02-11, & & & Ostermeir, Anna-Lena & P03-02 \\
\hline & $\begin{array}{l}\text { FV49, } \\
\text { P06-01 }\end{array}$ & & P07-05, & Nabring, Jens & P07-10 & Ott, Torsten & P08-03 \\
\hline
\end{tabular}




\section{P}

Pagel, Friederike

Pagel, Julia

Pansy, Jasmin

Patry, Christian

Paul, Pia

Paul, Thomas

Paulsen, Jennifer

Peng, Jenny

Pereira, Carina

Peter, Andreas

Peters, Harm

Petzoldt, Johanna

Picardi, Susanne

Pichler, Gerhard

Piening, Brar

Pilz-Klement, Ines

Platen, Christopher

Pocivalnik, Mirjam

Poets, Christian

$\begin{array}{ll} & \text { FV08, } \\ & \text { FV09, } \\ & \text { FV14, } \\ & \text { FV31, } \\ & \text { FV38, } \\ & \text { FV48, } \\ & \text { P01-07, } \\ & \text { P03-2, } \\ & \text { P03-06, } \\ & \text { P03-07, } \\ & \text { P05-10 } \\ & \text { P09-01, } \\ & \text { P09-10 } \\ & \text { P10-01, } \\ & \text { P13-07 } \\ \text { Pogorzelski, David } & \text { P07-03, } \\ & \text { P07-04 } \\ \text { Poppe, Andrea } & \text { P06-09 } \\ \text { Pöschl, Johannes } & \text { FV40, } \\ & \text { P03-01 } \\ \text { Pulzer, Ferdinand } & \text { FV41, } \\ & \text { FV43, } \\ & \text { P12-05 }\end{array}$

R

$\begin{array}{ll}\text { Rabenau, Holger } & \text { P08-08 } \\ \text { Rädecke, Jochen } & \text { P01-02 } \\ \text { Raith, Marco } & \text { FV08 } \\ \text { Raith, Wolfgang } & \text { P02-04 } \\ \text { Ramaswamy, Annette } & \text { P14-07 } \\ \text { Rasch, Christoph } & \text { P06-10 } \\ \text { Rathgeb-Maisch, R. } & \text { V01 } \\ \text { Razetti, Roberta } & \text { FV12 } \\ \text { Reichert, Jörg } & \text { FV28, } \\ & \text { P07-06, } \\ & \text { P07-07 } \\ & \text { P07-08 } \\ & \text { P07-09 }\end{array}$

FV23,

P04-01

P08-04

P04-06

P03-04

$\mathrm{P} 02-04$

FV02,

FV08,

FVo9,

FV31,

FV38,

48,

03-2,

03-07,

05-10,

13-07

P07-04

P06-09

FV40,

P03-01

FV43,

P12-05
Margarete $\quad \mathrm{P} 07-10$

FV26,

FV27

Reinhold, Anke P08-04

Reiss, Irwin FV49

Reister, Frank FV47, P02-

03

Rellensmann, Georg $\quad \mathrm{P} 08-03$

Renk, Hanna $\quad F V 44$, P11-

08

Renner, Marcus

P03-08,

P03-09

Repa, Andreas

P09-04,

P09-06

P02-08

Reschke, Stefan

P04-06

Retzlaff, Anne

P04-08

Reutter, Heiko P05-05,

P09-07

Riede, Frank-Thomas $\quad \mathrm{P} 14-05$

Rieger-Fackeldey, EstherFV10,

03-06,

09-01,

P10-01,

$$
\text { Ro }
$$

Rochow, Niels P09-02

Rödl, Siegfried P11-05

Roehr, Charles ChristophP02-02,

P02-09

Roeleveld, Nel P09-07

Rogosch, Tobias P13-03

Rolle, Udo P13-04

Rosewich, Hendrik $\quad$ P04-10

Rost, Imma P06-04

Rotering, Victoria Maria FV15,

P11-03,

P11-06

Roth, Bernhard P05-06,

FV28,

FV30,

P02-06,

P07-06,

P07-07,

P07-08,

P07-09,

$\mathrm{P} 10-06$

Rüegger, Christoph $\quad$ P05-04

\section{$S$}

Sadeghi, Kambis P03-05

Sahlmen, Silke

Salamano, Eduardo

$\mathrm{P} 07-10$

P10-02,

Sandejas, Darren P01-08

Sauerzapf, Elisabeth P09-06

Schäfer, Christoph P06-06

Schäfer, $H$. P04-09

Schäfer, Kristine P08-08

Schäfer, Stefan $\quad \mathrm{P} 11-07$

Schaffelder, Regine P02-08

Schaible, Thomas

\begin{tabular}{|c|c|}
\hline & $\begin{array}{l}\text { FV27, } \\
\text { P02-08 }\end{array}$ \\
\hline Scheffner, Thomas & P08-07 \\
\hline Schemken, Maryan & FV28 \\
\hline Scheuer, Till & P12-01 \\
\hline Schiffmann, Jan-Holger & P11-07 \\
\hline Schlensak, Christian & P11-08 \\
\hline Schleußner, Ekkehard & $\begin{array}{l}\text { FV05, } \\
\text { FV06 }\end{array}$ \\
\hline Schlößer, Rolf & $\begin{array}{l}\text { P08-05, } \\
\text { P08-08, } \\
\text { P13-03, } \\
\text { P13-04 }\end{array}$ \\
\hline Schlunk, Christian & P11-04 \\
\hline Schmalisch, Gerd & P02-02 \\
\hline Schmidt, A. & P08-02 \\
\hline Schmidt, Beatrix & P14-10 \\
\hline \multirow[t]{3}{*}{ Schmidt, Lisa } & FV18, \\
\hline & $\begin{array}{l}\text { FV21, } \\
\text { P12-07 }\end{array}$ \\
\hline & P12-08 \\
\hline Schmidtke, Susanne & P10-08 \\
\hline
\end{tabular}

Schweizer, Stephanie

S04-05,

Seipolt, Barbara $\quad$ FV30

Seitz, Guido P13-01

Selvaganapathy, Ravi P01-08

Siegel, Jens $\quad$ FV36

Siew, Melissa P02-09

Silver, Gabrielle P13-06

Simon, Arne FV41

Singer, Dominique P04-09,

P10-08,

Singh, Balpreet P09-03

Soditt, Volker Pfl.PO-03

Sommerfeld, Marion P07-06

Sostmann, Kai P10-09

Speer, Christian P. FV38,

P01-06,

P06-01,

P12-02

Sperandio, Markus $\quad$ FV32

Spiegler, Juliane $\quad \mathrm{P} 05-02$

Spittler, Andreas P03-05

Spring, Bärbel P03-02,

Schmitt, Jochen FV28,

P07-09

Schmitt, Anne Pfl.PO-06

Schmitz, Bernd P04-03

Schmitz, Thomas P08-04,

P12-01,

P14-02

Schmölzer, Georg $\quad F V 19$,

FV22,

FV23,

P10-04

Schnaufer, Lukas P10-01

Schneider, Hans-Christoph

P01-04,

P08-07

Schoberer, Mark FV15,

P05-03,

P11-02,

P11-03,

P11-06

Schönbeck, Marco P04-06,

P04-08

Schröder, Lukas $\quad$ FV35

Schüller, Simone P06-03

Schulze, Andreas FV24,

FV25,

P06-04,

P10-03,

P12-03,

P13-02

Schulze, Lizzy $\quad \mathrm{P} 14-10$

Schulzke, Sven P05-04

Schumacher, Johannes P09-07

Schumann, Stefan P06-06

Schütz Hämmerli, Natascha

$\begin{array}{ll} & \mathrm{Pfl} . \mathrm{PO}-05 \\ \text { Schützle, Heike } & \mathrm{P} 04-04\end{array}$

Schwaberger, Bernhard FV19,

FV20,

FV22,

$\mathrm{FV} 23$,

P02-05

Schwarz, Christoph E. FV17, P05

10
Springer, Wolfgang

Stangl, Oliver

Staudt, Martin

Stavropoulou, Dimitra

Stehen, Claudia

Stein, Anja

P03-06

FV29

P14-01

P12-06

P06-05,

P06-06,

P14-06

P14-10

FV36,

FV39,

FV41,

P02-10,

P05-01

Steinau, Gerd $\quad$ P11-02

Steinmann, Jörg $\quad$ FV36

Stoffel, Liliane Pfl.PO-05

Stoll, Lenja $\quad$ FV44

Storbeck, Tobias FV16,

Storz, P05-01

Strahleck, Thomas Pfl.PO-02

Strauss, Alexander $\quad F V 07$

Syrbe, Steffen

P14-05

T

te Pas, Arjan $\quad \mathrm{P} 02-07$

Tekaat, Maria $\quad$ FV11

Tenbrock, Klaus P03-03

Teufel, Manfred P14-09

Thanhäuser, Margarita P09-04,

P09-06

Thiede, Uwe P10-08

Thiel, Michael P06-10,

P07-01

Thio Lluch, Marta $\quad \mathrm{P} 02-09$

Thomas, Sumesh P09-03

Thomas, Wolfgang FV38

Thome, Ulrich FV43, P12-

05

Thyrian, Rene $\quad$ FV37

Tibboel, Dick P09-07 


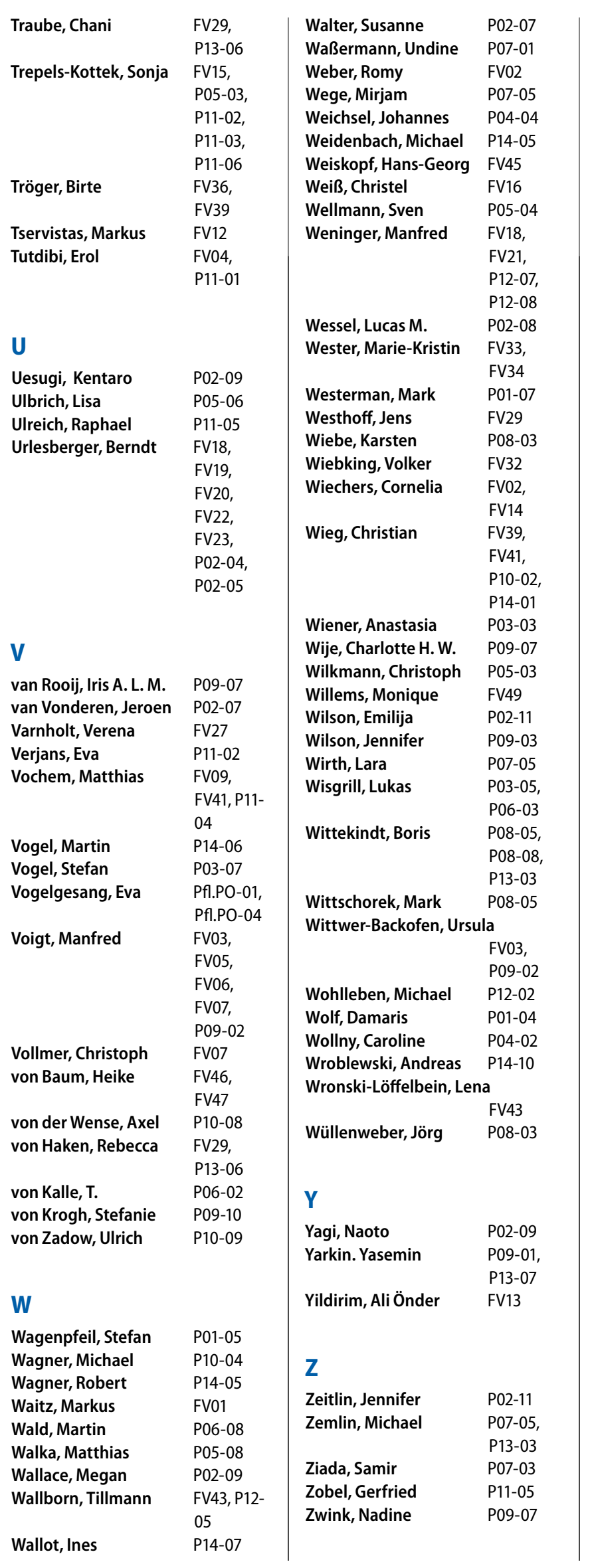

